\title{
Diabetes-related factors and atherosclerosis regression
}

Citation for published version (APA):

Josefs, T. (2020). Diabetes-related factors and atherosclerosis regression. [Doctoral Thesis, Maastricht University]. Gildeprint Drukkerijen. https://doi.org/10.26481/dis.20200903tj

Document status and date:

Published: 01/01/2020

DOI:

10.26481/dis.20200903tj

Document Version:

Publisher's PDF, also known as Version of record

\section{Please check the document version of this publication:}

- A submitted manuscript is the version of the article upon submission and before peer-review. There can be important differences between the submitted version and the official published version of record.

People interested in the research are advised to contact the author for the final version of the publication, or visit the DOI to the publisher's website.

- The final author version and the galley proof are versions of the publication after peer review.

- The final published version features the final layout of the paper including the volume, issue and page numbers.

Link to publication

\footnotetext{
General rights rights.

- You may freely distribute the URL identifying the publication in the public portal. please follow below link for the End User Agreement:

www.umlib.nl/taverne-license

Take down policy

If you believe that this document breaches copyright please contact us at:

repository@maastrichtuniversity.nl

providing details and we will investigate your claim.
}

Copyright and moral rights for the publications made accessible in the public portal are retained by the authors and/or other copyright owners and it is a condition of accessing publications that users recognise and abide by the legal requirements associated with these

- Users may download and print one copy of any publication from the public portal for the purpose of private study or research.

- You may not further distribute the material or use it for any profit-making activity or commercial gain

If the publication is distributed under the terms of Article $25 \mathrm{fa}$ of the Dutch Copyright Act, indicated by the "Taverne" license above, 


\section{Diabetes-related factors and atherosclerosis regression}


@ Tatjana Josefs, Maastricht 2020

All rights reserved. No part of this book may be reproduced or transmitted in any form or by any means (electronic, mechanical, photocopying, recording or otherwise) without prior written permission of the author, or when appropriate, by the publishers of the publications.

Cover Design: Guus Gijben

Printed by: Gildeprint

ISBN: 978-94-6402-483-8 


\title{
Diabetes-related factors and atherosclerosis regression
}

\author{
DISSERTATION \\ to obtain the degree of Doctor at the Maastricht University, on the authority of the \\ Rector Magnificus, Prof. Dr. Rianne M. Letschert, in accordance with the decision of \\ the Board of Deans, to be defended in public on
}

Thursday, September 3, 2020 at 12pm in Maastricht

by

Tatjana Josefs 


\section{Promoters:}

Prof. Dr. C.G. Schalkwijk (Maastricht University)

Prof. Dr. E.A. Fisher (NYU School of Medicine)

\section{Co-Promoter:}

Dr. K. Wouters (Maastricht University)

\section{Assessment Committee:}

Prof. Dr. C. Reutelingsperger (Chair, Maastricht University)

Prof. Dr. E.A.L. Biessen (Maastricht University)

Prof. Dr. M.P.J. de Winther (Amsterdam UMC)

Dr. M. Westerterp (University of Groningen)

Dr. R. Koenen (Maastricht University)

The research of this thesis was supported by a predoctoral fellowship of the American Heart Association (18PRE33990436). Financial support for the publication of this thesis by the Maastricht University is gratefully acknowledged. 


\section{Table of contents}

$\begin{array}{lll}\text { Chapter } 1 & \text { General Introduction } & 7\end{array}$

Part1:

Chapter 2 High-density Lipoprotein Cholesterol Efflux Capacity is not associated with Atherosclerosis and Prevalence of Cardiovascular Outcome: The CODAM Study

Chapter 3 Role of LpL (Lipoprotein Lipase) in Macrophage Polarization In Vitro and In Vivo

Chapter 4 Reduced High-density Lipoprotein (HDL) due to Hypertriglyceridemia does not affect Atherosclerosis Regression

Part 2:

Chapter $5 \quad$ Neutrophil extracellular traps (NETs) Promote MacrophageInflammation and Impair Atherosclerosis Resolution in Diabetic Mice

Chapter 6 Loss of DNase1, DNase1L3 or both does not significantly change atherosclerotic Neutrophil extracellular traps (NETs) content, or affect Atherosclerosis

Chapter 7 Summary \& General Discussion

Valorisation

Acknowledgements

Curriculum Vitae

Scientific Output

Abbreviations 
General Introduction 



\section{Diabetes and Cardiovascular Diseases (CVD)}

Type1 \& Type2 Diabetes Mellitus (T1DM, T2DM) are common diseases with increasing prevalence worldwide. ${ }^{1}$ In the United States alone, diabetes is the most expensive chronic disease, reaching about $\$ 327$ billion in $2017 .^{2}$ The global costs of diabetes are estimated around $\$ 727$ billion. ${ }^{3}$ Subjects suffering from diabetes, with the majority being diagnosed as $\mathrm{T}^{2} \mathrm{DM}^{4}$, have a significantly increased risk of atherosclerosis and associated cardiovascular events (CVE). ${ }^{5}$ This is due to the fact that metabolic risk factors for atherosclerotic CVD are commonly found in subjects with diabetes. Hyperglycemia is an independent risk factor for CVD, next to genetic predisposition, hypertension and diabetic dyslipidemia. The adjusted relative risk for T2DM compared to non-T2DM patients is 1.7 for cardiovascular death, 1.8 for myocardial infarction, and 1.5 for stroke. ${ }^{6}$

Cardiovascular diseases (CVDs) are the deadliest disease worldwide and despite a steady decline in death from CVD due to advances in scientific discoveries, clinical cardiology as well as public health, it is still responsible for $30 \%$ of deaths. ${ }^{7}$ The global cost is estimated to be $\$ 863$ billion and is predicted to increase by $\$ 1044$ billion until $2030^{8}$ due to a rising and aging population and the high incidence of secondary complications after survival of CVD incident.

\section{Atherosclerosis}

Atherosclerosis is the underlying vascular pathology of myocardial infarction (MI) and stroke, the two main CVDs, and describes a chronic-inflammatory disease that involves the formation of lesions (=plaques) in medium-sized to large arteries. ${ }^{9}$ Atherosclerotic plaque progression begins in childhood ${ }^{10}$ and progresses silently before symptoms occur. A key initiating step of atherosclerosis is the deposition of apoB-containing lipoproteins in the intima, followed by accumulation of cholesterol, immune cells, foam cells, and smooth muscle cells leading to the progression of atherosclerotic plaques. ${ }^{11}$ By the age of $40,95 \%$ of people have some lesions and problems occur if the lesions lead to reduced blood flow or ruptures and occludes vessel, thereby interfering with tissue oxygenation. ${ }^{12}$ Plaque rupture and thrombus 
formation preceding acute coronary events such as $\mathrm{Ml}$ and stroke are thought to be its main cause and the result of vulnerable (=rupture-prone) plaques. ${ }^{13}$ Prominent features of such vulnerable plaques (often referred to as thin-cap fibroatheroma) are large lipid- and macrophage-rich cores and necrotic areas covered by a thin fibrous cap. ${ }^{14}$ Additional characteristics associated with plaque vulnerability are the presence of cholesterol crystals, microcalcifications and intraplaque angiogenesis. ${ }^{14}$

Atherosclerosis regression describes the repair of blood vessels initiated by markedly reduced circulating cholesterol levels in animals and in humans ${ }^{15}$; cholesterol and macrophages leave the arterial wall and are replaced by collagen, reducing plaque vulnerability. Given that many patients start treatment once lesions have already formed, attaining atherosclerosis regression is an unmet, highly desirable, clinical goal.

Autopsy ${ }^{16-18}$ and clinical studies have confirmed the ability of human atherosclerotic plaques to regress. In clinical studies, plaque size and later arterial wall thickness (assumed to be equivalent to plaque volume) was measured using angiography or intravascular ultrasonography. However, reported changes in plaque size/volume ${ }^{19-23}$ are rather small compared to $\sim 80 \%$ reduction in clinical events in men with coronary artery disease (CAD) and $\sim 50 \%$ in individuals suffering from the metabolic syndrome with CAD. ${ }^{20}$ The modest quantitative changes suggest that changes in plaque composition might pre-dominate over change in plaque size, as already reported by Brown et al. in 1993. ${ }^{24}$ In contrast, results from the GLAGOV trial, which reported a $\sim 1 \%$ regression in plaque volume with addition of a proprotein convertase subtilisin/kexin type 9 (PCSK9 inhibitor) to statin therapy, did not find changes in plaque composition compared to statin monotherapy. ${ }^{25}$ Further studies and especially advances in imaging modalities ${ }^{26,27}$ to establish the predictive ability of plaque composition and CVD risk are needed.

As current techniques to evaluate regression in humans are not optimal, the study of regression in experimental animal models is of high significance. Animal models of atherosclerosis regression, many pioneered by the Fisher laboratory, have significantly increased the understanding of the mechanisms underlying the resolution of lesions and show that regression does not simply involve the same mechanisms as atherosclerosis progression just in reverse order, but has its distinct processes. A couple of recent reviews describe the underlying mechanisms of atherosclerosis regression in detail. ${ }^{15,28-30}$ Further, processes leading to a stable plaque such as 
deposition of collagen matrix can hinder a detection in reduced lesion size. Therefore, in animal models we have characterized atherosclerosis regression as not necessarily a reduction in lesion size, but as a reduction in lipid and/or macrophage content, a switch from a proinflammatory to a pro-resolving macrophage, smaller necrotic core size, and an increase in collagen.

\section{Mouse Models to study atherosclerosis regression}

Mice are resistant to atherosclerosis, which is likely due to their relatively low low-density lipoprotein cholesterol (LDL-C) levels and the fact that most of their plasma cholesterol is carried by high-density lipoproteins (HDL). ${ }^{15}$ To study atherosclerosis in mice, two genetically modified mouse models were developed in the early 1990s to hinder efficient lipoprotein clearance and thereby increase plasma cholesterol levels leading to atherosclerosis progression - 1. The apolipoprotein $\mathrm{E}$ deficient $\left(\mathrm{ApoE}^{-/-}\right)$ mouse model was created by the Breslow ${ }^{31}$ and Maeda ${ }^{32}$ laboratories; 2. The low-density lipoprotein deficient $\left(L d l r^{-}\right)$mouse model was developed by the Brown and Goldstein laboratory ${ }^{33}$. Feeding a western diet (WD; high fat, high cholesterol) can accelerate the development of atherosclerotic plaques in these models. ${ }^{15}$

There are several mouse models to study atherosclerosis regression, which are based on lowering LDL, raising HDL or treatment with anti-miR33 ${ }^{35}$. LDL-lowering strategies include re-expression of ApoE in $\mathrm{ApoE}^{-/-}$mice ${ }^{36,37}$ and Ldlr in $L d l r^{/-}$mice ${ }^{33}$ using an adenovirus; transplanting a plaque burdened thoracic arch or aortic arch segment to normolipidemic animals ${ }^{38}$; Reversa mouse with conditional lowering of LDL cholesterol $\left(\text { Ldll }^{/-} \mathrm{Apob}^{100 / 100} \mathrm{Mttp}^{f / f l} \mathrm{Mx} 1 \mathrm{Cre}^{+/+}\right)^{39}$; and switching Ldl/r- from WD to chow $\operatorname{diet}^{40}$. Novel strategies have been developed to study regression in any mouse model, not being limited to mice with a genetic background of $\mathrm{Ldll}^{-/}$or $A p o E^{-/}$. These methods first induce LDLr deficiency using PCSK9-adenovectors ${ }^{41}$ or LDLrASO ${ }^{42}$, followed by induction of regression using antisense oligonucleotides against $\mathrm{ApoB}^{43}$ or microsomal triglyceride transfer protein (MTTP) ${ }^{41}$. Raising HDL particle levels is achieved either genetically ${ }^{44}$ or by infusion ${ }^{45}$ of apoA1 or HDL respectively. Space does not permit full description of all atherosclerosis regression models but will be focused on the ones used in this Thesis and are all based on lowering LDL.

The aortic transplant mouse model was pioneered by the Fisher laboratory in 
the early 2000s and involves the transplantation of an atherosclerotic arch from a hyperlipidemic donor mouse (e.g. ApoE-/- or Ldlr/-) into a normocholesterolemic recipient mouse, interpositioned with the abdominal aorta and blood flow directed through the graft. This leads to a rapid environmental change and is not of transient nature as some other atherosclerosis regression models, hence leading to plaque regression over a surprisingly short time (<1 week).

In 2013, the Fisher laboratory showed that atherosclerosis regression could also be achieved by switching an $L d l r^{\prime-}$ mouse from a WD to a chow diet over an extended period without any intervention. ${ }^{40}$ This method, however, is slower and is variable in its effectiveness. ${ }^{46}$

A novel regression mouse model is using a combination of LDLR antisense and sense oligonucleotides (ASO; SO) and was pioneered by the Goldberg laboratory in collaboration with the Fisher laboratory. In this model, LDL-C is increased by the injection of LDLR ASO, leading to a similar phenotype as $\mathrm{Ldll}^{/-}$mice. GalNAc-conjugated SO are designed to bind and inactivate the LDLR ASO, and accelerates the return of LDLR expression and normal LDL-C levels.

Another novel mouse model is the PCSK9-adenovirus in combination with an ApoB ASO (MTTP inhibitor, which reduces very low-density lipoprotein (VLDL) secretion, is also an option). PCSK9 directs hepatic LDL receptors for degradation ${ }^{47}$ and thereby reduces LDL receptor expression, hence increasing plasma LDL cholesterol (LDL-C). ApoB ASO reduces hepatic apoB mRNA expression, hence reduces VLDL secretion and decreased plasma cholesterol levels by about $90 \% .{ }^{43}$

Diabetes-associated factors are thought to contribute to the increased risk of developing CVD in individuals suffering from diabetes compared to non-T2DM individuals. Two of these factors are elevated TG levels, a feature of diabetic dyslipidemia, and hyperglycemia-induced neutrophilia. This Thesis is divided into two parts to study each of these factors in regard to atherosclerosis regression.

\section{Triglycerides and their role in atherosclerosis}

The question if triglycerides (TGs) are atherogenic has vexed clinicians for more than half a century and is still debated. Already in 1973 Goldstein et al. reported that 
elevated TG levels are common in CVD patients ${ }^{48}$ and in the early 1980s, LDL-C and TG were seen as equal contributors to atherosclerosis. ${ }^{49}$ The role of LDL-C in CVD has been largely established, including by the Nobel prize winning discoveries by Brown and Goldstein in 1985 leading towards the development of the LDL-C lowering drug class called statin (3-hydroxy-3-methylglutaryl coenzyme A (HMG-CoA) inhibitor). Statin-induced LDL-C reduction lowered clinical events and all-cause mortality (e.g. primary prevention: JUPITER Trial ${ }^{50}$, secondary prevention: PROVE IT-TIMI 2251). While mean LDL-C levels in the population declined from 1999 to $2016^{52}$, TG levels have been increasing with the epidemic of obesity and diabetes. Further, although LDL-lowering reduced CVD risk and mortality, there is still residual risk of up to $70 \%$. People with diabetes especially do not respond as robustly to lipid lowering therapies as non-diabetics, and even after lipid-lowering sustain increased risk. ${ }^{53,54}$

Despite studies showing an association of elevated circulating TG-rich lipoproteins with increased CVD risk, it is still unclear if this is due to direct toxic effects on arteries or whether this is merely an association. It is challenging to disentangle the role of TG in CVD risk because elevated plasma TG levels are always accompanied by other factors that themselves are plausibly causally related to CVD, e.g. low HDL-C and high small dense LDL-C levels in diabetic dyslipidemia ${ }^{55}$ as well as non-lipoprotein parameters such as hypertension, a proacoagulant state, hepatosteatosis, or abdominal obesity ${ }^{56}$. This uncertainty also led to a lack in treatment options for elevated TG and currently TG treatment is not included in the latest recommendations to lower CVD risk in the United States and optional in Europe. Given the increasing evidence that TG-rich lipoproteins can cause CVD and are not only an innocent bystander, further research is needed to finally disentangle the role of TGs in CVD.

\section{Definition of hypertriglyceridemia(HyperTG)}

The standard practice to measure TG levels is after $12 \mathrm{~h}$ of fasting. Fasting TG levels of $>1.7 \mathrm{mmol} / \mathrm{l}$ (>150mg/dL) are generally accepted to be classified as HyperTG and can be further classified into borderline high (1.8-2.2mmol/L,150-199mg/dL), high (2.3-5.6mmol/L, 200-499mg/dL), and very high

\begin{tabular}{|l|cc|}
\hline \multicolumn{3}{|c|}{ TG } \\
\hline & $\mathrm{mmol} / \mathrm{L}$ & $\mathrm{mg} / \mathrm{dL}$ \\
Normal & $<1.7$ & $<150$ \\
borderline high & $1.8-2.2$ & $150-199$ \\
high & $2.3-5.6$ & $200-499$ \\
very high & $>5.7$ & $>500$ \\
\hline
\end{tabular}

Table 1. Classification of circulating triglyceride (TG) levels according to the national lipid association 
(>5.7mmol/L, >500mg/dL) according the National Lipid Association (Table 1). ${ }^{57}$ The prevalence of HyperTG in the USA was $47 \%$ in 2010 , which was largely driven by lifestyle factors. ${ }^{58}$ While in Europe, $27 \%$ had nonfasting TG levels of $>2.0 \mathrm{mmol} / \mathrm{L}$ in 2016. ${ }^{49}$ Off note, nonfasting TG levels measured 1-6h after meals only change $0.2 \mathrm{mmol} / \mathrm{L}(8 \mathrm{mg} / \mathrm{dL})$ compared to fasting TG levels, leading to the suggestion from the European Atherosclerosis Society to assess the plasma lipid profile in nonfasting blood. ${ }^{59}$ Further, both non-fasting and fasting plasma TG levels have been associated with CVDs.

\section{TG metabolism \& causes of HyperTG}

TGs are carried within chylomicrons and VLDL (=TG-rich lipoproteins, or TRL) and contain exogenous and endogenous TGs (Figure 1). Exogenous TGs enter the human body via the diet and together with apoB48 they are packed into chylomicrons within enterocytes. Chylomicrons are the largest lipoprotein particles and have a TG content of about $80-95 \%$. Through interaction of chylomicrons with HDL particles in the intestinal lymph capillaries, chylomicrons acquire apoC-II, apoC-III, and apoE on their way through the lymphatic system until they enter the subclavian vein via the thoracic duct. Endogenous TG are synthesized in hepatocytes by glycerol-3-phosphate dehydrogenase using free fatty acids (FFA) and glycerol and form together with apoB100 VLDL particles and obtain apoC-I, apoC-II, apoC-III, and apoE during secretion from hepatocytes.

Both, chylomicrons and VLDL are hydrolyzed by lipoprotein lipase (LpL) on capillary endothelial cells in peripheral tissues, resulting in FFA and chylomicron remnants/smaller VLDL particles followed by intermediate-density lipoproteins (IDLs). FFA can enter cells and either be resynthesized into TGs and stored in adipose tissue (AT), or they can be oxidized, mainly in mitochondria, by various cell types for energy retrieval. Chylomicron remnants can be cleared by the LDLr or LDLr-related proteins on hepatocytes. IDLs can be similarly taken up by hepatocytes and catabolized or can undergo further hydrolysis by LpL or hepatic lipase forming LDL particles. Further changes in VLDL and LDL is done by cholesterol ester transfer protein (CETP), which exchanges TG and cholesteryl ester (CE) between these lipoproteins and HDL.

HyperTG is the result of an increase in TG-rich lipoproteins due to increased 
synthesis of TG-rich lipoproteins, decreased catabolism, or the combination of both. The most common form of HyperTG is mild-to-moderate HyperTG $(1.8-5.6 \mathrm{mmol} / \mathrm{L})$ which is characterized by an increase in primarily VLDL and its remnants ${ }^{60}$. Mild-tomoderate HyperTG can be due to polygenic traits in the LpL gene and related factors (e.g. GPIHBP1, APOC2, APOA5, GPD1, LMF1). HyperTG also often occurs as a secondary event in patients with the metabolic syndrome and/or T2DM as part of the diabetic dyslipidemia (Figure 1). In T2DM, an insulin resistant state, the production of VLDL is increased while the hepatic uptake of VLDL, IDL and LDL is decreased. This together with a reduction in LpL activity results in an increase in circulating TRLs. The large amount and prolonged time of TRLs in the circulation drives the CETP reaction, leading to TG-enriched LDL and HDL that are more readily hydrolyzed by hepatic lipase $^{61}$ and endothelial lipase ${ }^{62}$ and smaller HDL particles are cleared faster by the kidney. The result is atherogenic small dense LDL particles, reduced HDL-C concentrations and elevated TG levels - diabetic dyslipidemia. Further, HyperTG can be the result of unhealthy diet or lifestyle choices such as alcohol overuse, lack of exercise, hepatosteatosis, hyperthyroidism, pregnancy, nephrotic syndrome, certain medications such as glucocorticoids and weight regain after substantial loss. ${ }^{56}$

Severe HyperTG is ( $>5.7 \mathrm{mmol} / \mathrm{L})$ mainly associated with an increase in chylomicrons, hence also called chylomicronemia. The very rare monogenic chylomicronemia (also called familial chylomicronemia) is mainly caused by homozygous mutations in LpL gene (>90\%; Figure1) or its cofactors ${ }^{63}$ and leads to HyperTG by an increase of chylomicrons only. ${ }^{64}$ Polygenic chylomicronemia is more common and, in contrast to monogenic chylomicronemia, is not only associated with an increase in chylomicrons, but also with an increase in VLDL, and remnants of both. ${ }^{63,65}$ Secondary factors, such as an unhealthy lifestyle, can exacerbate TG levels. 


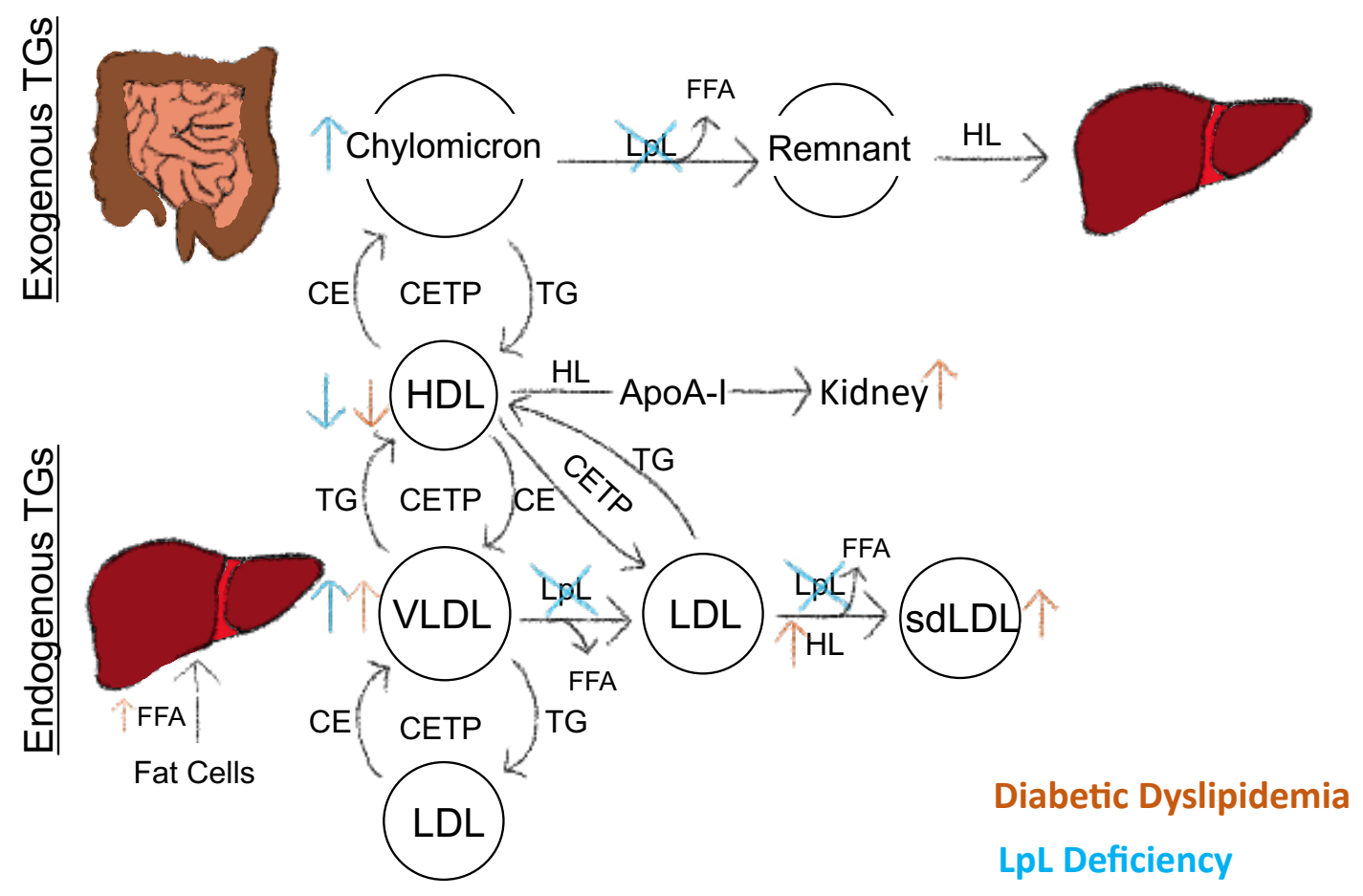

Figure 1. Simplified illustration of the TG metabolism and two causes leading to hypertriglyceridemia

TG, Triglyceride; FFA, free fatty acid; HL, hepatic lipase; CE, cholesteryl ester; CETP, cholesteryl ester transfer protein; HDL, high-density lipoprotein; VLDL, very low-density lipoprotein; LDL, low-density lipoprotein; sdLDL, small dense LDL; LpL, lipoprotein lipase

\section{Hypertriglyceridemia and CVD}

Lipid-lowering agents, especially statins, have lowered CVD risk by $25-35 \%{ }^{66}$ However, a prospective meta-analysis of data from 90,056 participants showed that there was a residual CVD risk in patients with preexisting CVD treated with statins that was not due to LDL-C, suggesting that it might be related to other lipoproteins such as TRL or HDL. ${ }^{67}$ One of the first large studies describing the association of TG with CVD was the Framingham study in $1986 .{ }^{68}$ Since then, the perception of TG's role in CVD has fluctuated over the past decades from being the cause of CVD to only being an innocent bystander. This fluctuation is mainly due to TG's close relationship with HDL-C (due to changes in LpL and CETP actions, see Figure1). Due to the fact that HDL-C and TG are so closely associated, it remained unclear which of these two lipid fractions were the real cause of increased CVD risk and a number of studies failed to show an independent association of TG with CVD risk. These studies report that after 
adjustment for other risk factors, especially HDL-C, TG did not add any additional predictive value. ${ }^{69-72}$ Factors making it difficult to detect an independent relationship could be the small numbers of participants of some of the studies, the TG measurement itself, which is known to be difficult because of the high variability of TG levels in a single individual, including the lack of its correction. ${ }^{73-78}$ At the same time, low HDL-C was independently associated with increased CVD risk ${ }^{79-81}$. However, with HDL-C raising trials failing to show benefits on CV outcome and additional evidence from epidemiological and genetic studies, TG and remnant cholesterol might be more likely the cause for CVD risk rather than HDL-C. ${ }^{82}$

Evidence for the independent role of $T G$ in CVD is growing and indicates that individuals with HyperTG are at increased risk to develop CVD. ${ }^{83}$ This has been shown in meta-analyses ${ }^{67,84-86}$, case-control studies ${ }^{79,87-92}$ and genetic studies ${ }^{93-95}$. In 2007, a meta-analysis of the western population including 262,525 patients, an odds ratio of $1.72(95 \% \mathrm{Cl} 1.56-1.90)$ for incident CVE has been reported for patients in the top tertile of TG levels after adjusting for common risk factors. ${ }^{85}$ This odds ratio is similar to the one observed in the meta analyses of the Asian and Pacific population ${ }^{84}$. The Copenhagen City Heart Study showed that for individuals with severe HyperTG (nonfasting, $>6.6 \mathrm{mmol} / \mathrm{L} ; 580 \mathrm{mg} / \mathrm{dL}$ ) the risk for myocardial infarction was 5.1 -fold increased compared to individuals with normal TG levels $(0.8 \mathrm{mmol} / \mathrm{L} ; 70 \mathrm{mg} / \mathrm{dL})$, 3.2-fold increased for ischemic heart disease, 3.2-fold increased for ischemic stroke, 2.2-fold increased for all-cause mortality. ${ }^{49}$ Interestingly, the risk for ischemic heart disease in women with elevated plasma TG levels is higher than for men. ${ }^{86,96,97}$ Another meta-analysis by Murad et al. included 35 studies with $>920,000$ subjects and reported an odds ratio of $1.80(95 \% \mathrm{Cl}, 1.31-2.49)$ for cardiac events, $1.31(95 \% \mathrm{Cl}$, 1.15-1.49) for myocardial infarction and 3.96 (95\%, Cl 1.27-12.34) for pancreatitis. ${ }^{98}$ TG is also an independent risk factor for CVD in individuals with diabetes ${ }^{99-102}$, the metabolic syndrome ${ }^{103-106}$ or familial combined hyperlipidemia ${ }^{107,108}$.

The most persuasive evidence for TG's causal role in CVD risk comes from recent genetic studies. In a large mendelian randomization trial of about 20,000 myocardial infarction cases and 50,000 control participants, a 1SD increase in TG levels was associated with a $54 \%$ increase in myocardial infarction risk, while low HDL-C showed no association. ${ }^{109}$ Further, increased TG levels due to variants in 
APOA5 increased the risk for coronary heart disease $(\mathrm{OR} 1.18,95 \% \mathrm{Cl}$ 1.11-1.26). ${ }^{110,111}$ A study using individuals from the Copenhagen City Heart Study with variants in the $L p L$ gene showed that a reduction in TG levels of $1 \mathrm{mmol} / \mathrm{L}(88.5 \mathrm{mg} / \mathrm{dL})$ was associated with a $50 \%$ reduction in all-cause mortality risk. ${ }^{95}$ Also variants in the APOC3 $3^{112,113}$ and ANGPTL ${ }^{114,115}$ genes point towards a casual effect of TG on CVD risk.

\section{Mechanisms of TG atherogenicity}

The exact mechanisms by which TG may promote atherosclerosis remain to be elucidated. Until recently, the general accepted view was that patients with HyperTG due to increased circulating chylomicrons were resistant to atherosclerosis ${ }^{116}$, because these lipoproteins were thought to be too large to transverse the endothelium. Animal models support the hypothesis that larger TG-rich lipoproteins are less atherogenic than smaller cholesterol-rich lipoproteins, however they are not totally harmless. While chylomicrons may be to be too big to enter the arterial wall, chylomicron remnants and VLDL and its remnants are able to enter arterial wall ${ }^{117}$ and are suitable for transcytosis in specialized clathrin-coated vesicles. ${ }^{118,119}$

Although patients with severe HyperTG (chylomicronemia) have low risk for atherosclerosis ${ }^{120,121}$, these subjects usually also have low LDL-C levels, likely contributing to the decreased CVD risk. However, for most of the population, HyperTG is in the range of mild to moderate HyperTG, tying increased atherosclerosis risk to smaller TG-rich particles and their remnants.

In 1973, Zilversmit hypothesized that postprandial increases in TG and elevated remnant lipoproteins cause atherosclerosis and proposed two possible mechanisms (Figure 2). ${ }^{122}$ Both are based on the hydrolysis of TG by LpL - one describes the atherogenic potential of remnant particles, the second one the lipolysis step itself. ${ }^{122}$ After crossing the endothelial barrier, remnants can be taken up by arterial macrophages directly ${ }^{123}$, contributing to foam cell formation. ${ }^{124,125}$ Additionally, TRL remnants carry more cholesterol per particle than LDL due to their size. ${ }^{126}$ For these reasons, remnants are seen as atherogenic and have been linked directly to plaque progression ${ }^{127}$, rather than TG themselves. ${ }^{82}$ The importance of remnants in CVD risk was also confirmed in a meta-analysis using the Emerging Risk Factors Collaboration 
data that detected TGs as a strong risk factor for CVD and stroke. ${ }^{72}$ This association attenuated after adjusting for HDL-C, but was lost after adjustment for non-HDL-C/remnants (VLDL, IDL, LDL). ${ }^{72}$ These remnant particles that can cause atherosclerosis are usually rapidly cleared by the liver. However, in individuals with T2DM/metabolic syndrome, hepatic clearance is delayed. Thus, prolonged exposure and increased concentrations raises the possibility for particles to enter atherosclerotic plaques. ${ }^{117,128}$ Additionally, the lipolysis step itself is thought to be atherogenic. LpL can hydrolyze TG into monoacylglycerols and FFA at the endothelial surface or within the arterial intima, which is associated with increased atherosclerosis and CVD. ${ }^{129}$ Overall, uptake and lipolysis of TG-rich remnants have been shown to promote endothelial dysfunction, foam cell formation, increase inflammation, damage smooth muscle cell, and impaired vascular repair. ${ }^{124,127,130,131}$

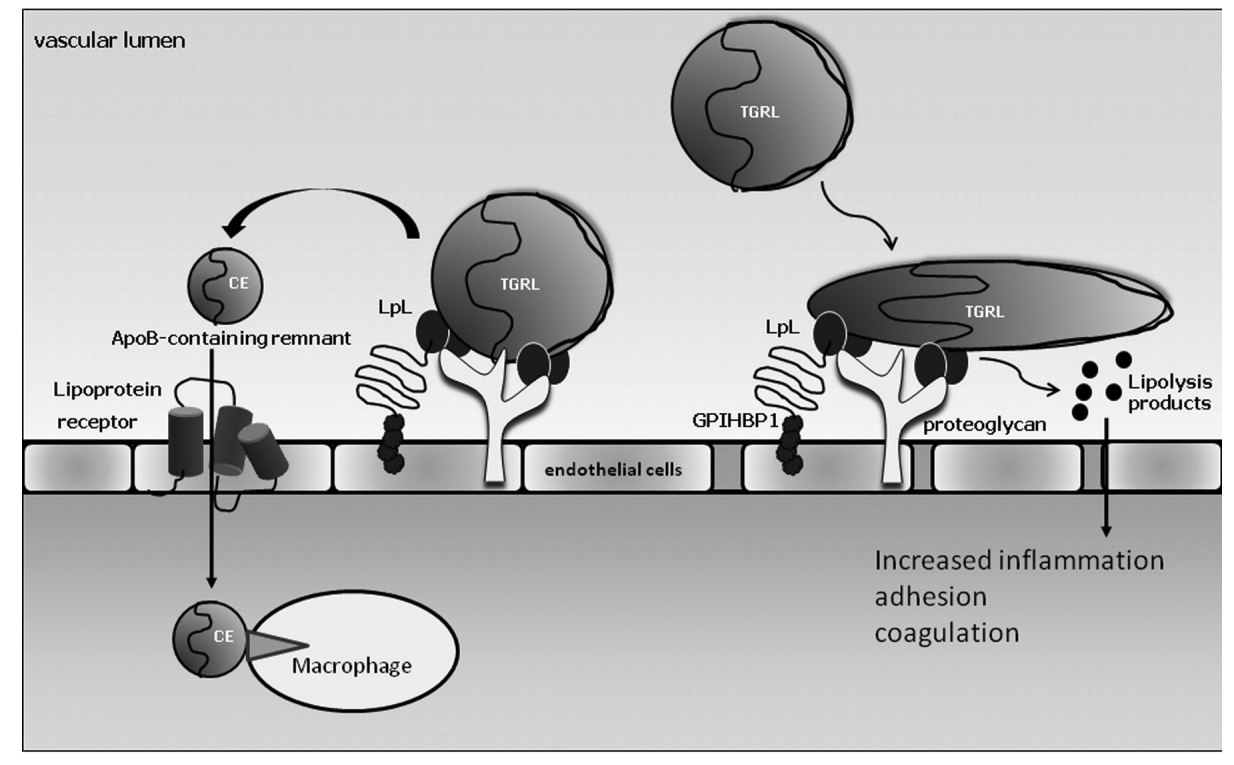

Remnant hypothesis

Lipolytic toxin hypothesis

Figure 2. Two possible mechanisms for the atherogenicity of triglycerides.

Illustration from Goldberg et al. Triglyceride and Heart Disease. Still a hypothesis? Arterioscler Thromb Vasc Biol. 2011;31:1716-1725

\section{Reducing TG levels \& Clinical Trials}

A key question is whether reducing TG levels is protective of CVD. So far, patients with TG levels $>500 \mathrm{mg} / \mathrm{dl}$ are considered at increased risk of pancreatitis ${ }^{57,132-134}$ and therefore receive TG lowering treatment. Regarding CVD, European Guidelines (ESC/EAS guidelines for the management of dyslipidemias ${ }^{135}$ and European 
guidelines on CVD prevention in clinical practice $\left.{ }^{136}\right)$ recommend that individuals with triglyceride levels $>1.7 \mathrm{mmol} / \mathrm{l}$ (>150 mg/dl) should be considered at increased risk of CVD, but did not include risk estimation of TG or recommended target values. Earlier guidelines from the adult treatment panel are in line with the European guidelines ${ }^{137}$, but more recent guidelines from the ACC/AHA on the assessment of CVD risk don't mention TGs at all. ${ }^{138}$ However, reducing TG could be beneficial for CVD, especially in individuals with preexisting coronary heart disease and diabetes. ${ }^{73,137,139,140}$

Several clinical trials of TG lowering have reported benefits for CVD risk. ${ }^{60,82,141}$ Medications that have shown efficacy in reducing TG levels are fibrates, statins, niacin and omega-3 PUFA. Fibrates lower TG by $30-50 \%$ depending on the baseline TG levels and are the most efficient TG lowering drug ${ }^{73}$. This TG reduction is due to an increase in FA oxidation, LpL synthesis and a reduction in apoC-III expression. A study using the fibrate gemfibrozil reduced CVD risk in patients with elevated TG levels. ${ }^{142,143}$ Further, a meta-analysis of 18 trials and 45058 patients reported a $10 \%$ relative risk reduction for major CVE, predominantly by prevention of coronary events, in individuals with HyperTG alone or in combination with HDL-C with fibrate intake. ${ }^{144}$ Also posthoc analyses of fibrate trials revealed that especially patients with low HDL-C (e.g. ACCORD trial $<34 \mathrm{mg} / \mathrm{dL}$ ) and elevated TG (e.g. ACCORD $>204 \mathrm{mg} / \mathrm{dL}$ ) levels as features of the metabolic syndrome and diabetes have CVD benefits from TG lowering using fibrates. ${ }^{145,146}$ Statins are used to lower LDL-C and have been shown to also reduce TG and non HDL-C by $10-15 \% .{ }^{147} \mathrm{~A}$ study of pravastatin in diabetics suggests that low HDL-C and elevated TG were significant predictors of increased CVD risk. ${ }^{148}$ Unfortunately, combination therapies of statins and fibrates have failed to show additional CVD risk benefits. ${ }^{149,150}$ Niacin is a B vitamin that reduces TG production by the liver ${ }^{151,152}$ and lowers TG levels depending on its dose up to $50 \%{ }^{153}$. Clinical trials failed to show a decrease in CVD event when niacin is added to statin treatment (AIM-HIGH ${ }^{66}$, HSP2-Thrive ${ }^{154}$ ). However, posthoc analyses of the AIM-HIGH Trial suggested that there might be a benefit in individuals with $T G>200 \mathrm{mg} / \mathrm{dL} .{ }^{155}$ Daily intake of 3-4mg/day of omega-3 PUFA (eicosapentaenoic acid (EPA) + DHA) from marine source, but not plant-derived, has been shown to reduce TG levels by $45 \%$ in individuals with HyperTG. ${ }^{156-159}$ A recently published Trial, the REDUCE-IT Trial, showed that in statin treatment patients with elevated TGs and CVD or diabetes, treatment with icosapent-ethyl, a highly purified EPA, reduces CVD risk including first, 
subsequent, and total ischemic events ${ }^{160,161}$. However, it is still unclear if the reduction in ischemic events was due to lowering TG or due to a combination of effects involving plaque instability reduction and inflammation. Notably, no differences in HDL-C upon icosapent-ethyl were detected.

Overall, clinical trials that appear to confirm CVD benefits of TG-lowering therapy rely on subgroup analyses and trials designed to focus on subjects with HyperTG only are missing. Thus, current results are non-conclusive. The REDUCE-IT trial was the first published trial to determine the relationship between TG and atherosclerosis in a population with elevated plasma TG levels and there are currently further clinical trials ongoing (e.g. STRENGTH) with results being expected by 2022 (Reviewed in ${ }^{162}$ ).

\section{Neutrophils and their role in atherosclerosis}

Neutrophils are polymorphonuclear cells and are, with about $75 \%$ of the leukocyte population, the most abundant immune cell in the adult human body. ${ }^{163}$ Leukocytes can be divided in subtypes (neutrophils, lymphocytes, monocytes, basophils, eosinophils) and are essential mediators of the immune system that derive from hematopoietic stem cells (progenitor cells). ${ }^{164}$ Hence, leukocytes are responsible for fighting against foreign elements and maintaining tissue homeostasis. ${ }^{165}$ Since a couple of decades, sterile inflammation by activation and aggregation of leukocytes have been implicated in chronic inflammatory disease ${ }^{166,167}$ and recent results of clinical trials have affirmed the inflammation hypothesis in CVDs (CANTOS, COLCOT). ${ }^{168,169}$

The relationship between CVD and leukocytes was first suggested over 80 years ago. ${ }^{170}$ Since then, CVD and diabetes have both been associated with leukocytosis ${ }^{171}$, including an association between increased risk of CVD with elevated neutrophil levels. ${ }^{172,173}$ Diabetes is characterized by neutrophilia and monocytosis and clinical studies as well as experimental mouse data suggest that hyperglycemia leads to abnormalities in neutrophil chemotaxis, phagocytosis and bacteriocidal properties ${ }^{40,174}$, while lowering blood glucose levels using anti-diabetic drugs has been shown to improve neutrophil activity. ${ }^{175}$ Neutrophils are present in human ${ }^{176}$ and mouse atherosclerotic lesions. ${ }^{177-180}$ Further, circulating neutrophil levels have been linked to atherosclerotic burden $40,177,181$ and neutrophil depletion reduces 
atherogenesis. ${ }^{180}$ In diabetic mice, reduction in circulating neutrophil levels by normalizing blood glucose levels was linked with improved atherosclerosis regression. ${ }^{40}$ In contrast, neutrophils ${ }^{182}$ and neutrophil extracellular traps (NETs) ${ }^{183}$ (discussed below) can also have beneficial effects in cardiac tissue healing after a myocardial infarction.

Despite all the evidence of the possible implication of neutrophils in the pathophysiology of CVD, neutrophils have been overshadowed by other leukocyte subtypes such as monocytes and macrophages, partly due to neutrophil's short life span with a half-life of approx. 6 to 8 hours in mice and humans. ${ }^{184}$ However, activated neutrophils have increased longevity, which leads to the presence of primed neutrophils at sites of inflammation. ${ }^{184}$ Further, recent discoveries showed that neutrophils contribute to chronic inflammatory disease via crosstalk with other cells. ${ }^{185,186}$ Especially the discovery of neutrophil's ability to form NETs, a process called NETosis, has sparked new interest in neutrophils under inflammatory conditions. ${ }^{187}$

\section{NETosis}

The process of NET formation can unfold in two distinct forms - 'suicidal' (also called Iytic) and 'vital' NETosis (Figure 3). ${ }^{188,189}$ Both forms differ in type of trigger, timing and mechanism of NETs release. 'Suicidal' NETosis requires a strong activation of nicotinamide adenine dinucleotide phosphatase (NADPH) oxidase over several hours, chromatin condensation via myeloperoxidase (MPO) and activation of neutrophil elastase (NE), resulting in neutrophil death. ${ }^{188,190}$ This form of neutrophil death is distinct from other forms of cell death such as apoptosis, necrosis or necroptosis. ${ }^{188}$ 'Vital' NETosis upon stimulation by, e.g. LPS, entails vesicular trafficking of DNA to the extracellular space without membrane perforation. It is a rapid process compared to 'suicidal' NETosis and results in a viable anuclear neutrophil. ${ }^{191-193}$

Irrespective of NETosis form, NETs consist of cytosolic and nuclear material including histones, NE, MPO, proteinase 3 (PR3), cathepsin and gelatinase. ${ }^{187}$ Upon stimulation by pathogens, such as bacteria or fungi, neutrophils expel NETs to catch pathogens thereby protecting the host.$^{187,194}$ Thus, NETosis belongs to one of several 
strategies (e.g. degranulation, phagocytosis) of neutrophils to respond to damage/infection. ${ }^{195}$

Our understanding of how NET formation is controlled is still limited. Several intracellular mechanisms and signaling pathways for NETs formation have been reported and recently reviewed in detail. ${ }^{196}$ The original observation of NETosis describes a phorbol myristate acetate (PMA)-induced reactive oxygen species (ROS) production via the NADPH oxidase. ${ }^{188}$ Downstream of ROS, peptidylarginine deiminase 4 (PAD4) and MPO lead to chromatin decondensation, a pivotal step of NETosis. ${ }^{190,197-200}$ Thereby PAD4 catalyzes the deamination of arginine residues of histone to citrulline. ${ }^{197-199}$ MPO promotes NE release from azurophilic granules ${ }^{201}$ from where NE translocates to the nucleus and partially cleaves histones. ${ }^{190}$ Recently, NE has been shown to also mediate the proteolytic activation of the pyroptosis-associated protein gasdermin D, which, along with capsase-11, mediate suicidal NETosis. ${ }^{202,203}$ Independently of the activation of the NADPH oxidase, also mitochondrial ROS formation can lead to NETosis via calcium influx. ${ }^{192,204,205}$ Notably, also other cell types like macrophages or mast cells can release decondensed chromatin - a process called ETosis. ${ }^{206,207}$

Next to being an important part of the hosts' immune defense, NETs have also been reported to be implicated in many chronic inflammatory disease such as rheumatoid arthritis ${ }^{208}$, cancer $^{209}$, diabetes ${ }^{210}$ and atherosclerosis ${ }^{211,212}$. 
Chapter 1

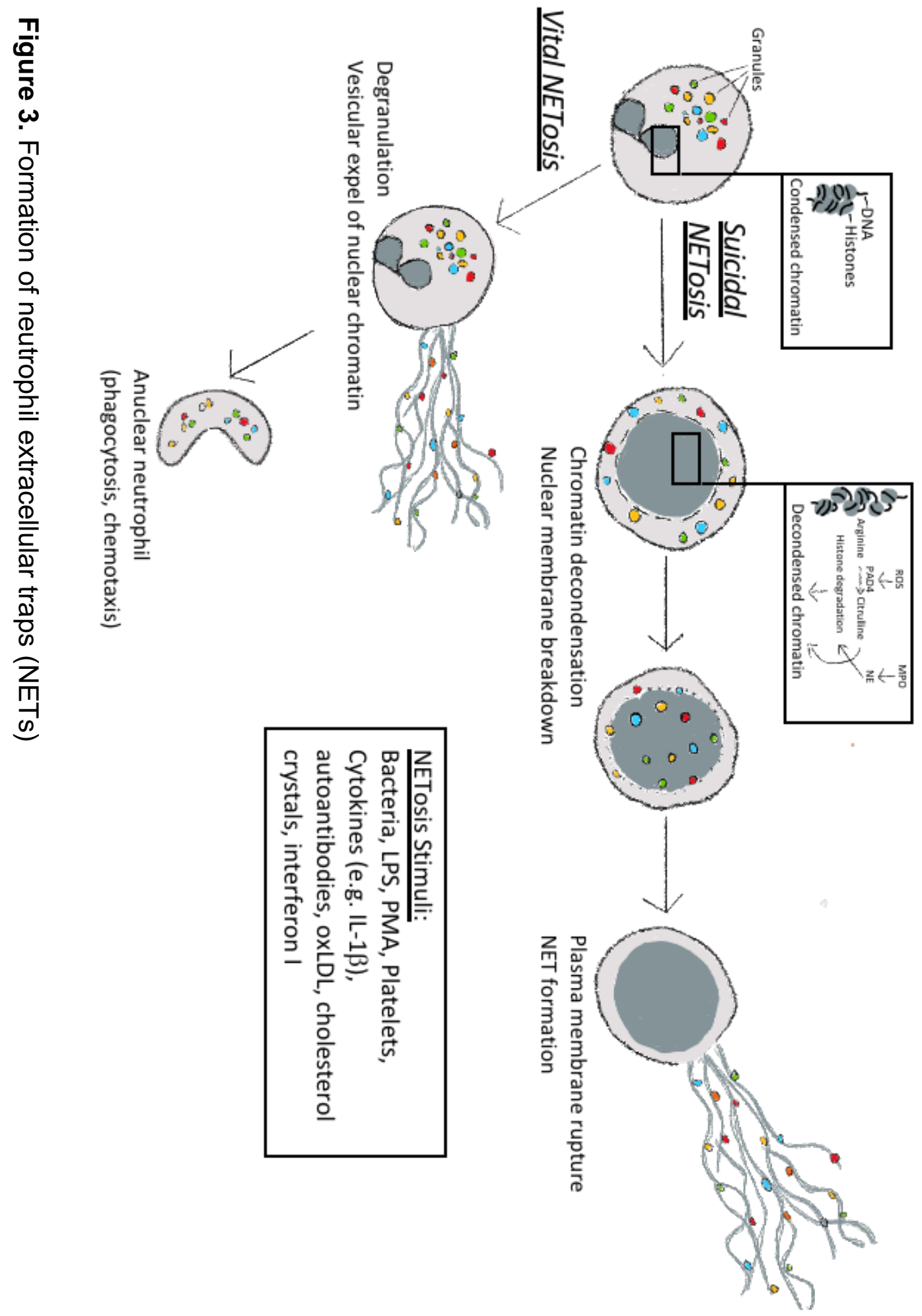




\section{NETosis and atherosclerosis}

NETosis in context of atherosclerotic disease can be stimulated by infectious triggers such as Tannerella forsythia ${ }^{213}$ and noninfectious triggers such as activated platelets ${ }^{214}$, oxidized LDL ${ }^{215}$ and cholesterol crystals ${ }^{216}$.

In humans, circulating NETs markers (double stranded DNA, nucleosomes, citrullinated histone H4, MPO-DNA complexes) have been independently associated with atherosclerotic lesion size severity of coronary artery disease, a pro-thrombotic state and the occurrence of major adverse cardiac events. ${ }^{217}$ NETs have been shown to be present in intact ${ }^{176,218,219}$ and complicated thrombotic plaques ${ }^{220,221}$ in humans as well as in atherosclerotic plaques of mice $216,222,223$. Interestingly, autopsied plaques from $\mathrm{Ml}$ patients revealed significantly more NETs in complicated plaques (e.g. superficial erosion or hemorrhage) compared to intact plaques. ${ }^{221}$

Experimental mouse studies revealed that genetic and pharmacological inhibition of NETs formation leads to reduced plaque size, inflammation and plaque stability. ${ }^{216,223,224}$ Within the plaque, NETs components and especially the interrelationship between neutrophils and macrophages have been demonstrated to modulate atherosclerotic plaques by recruiting inflammatory monocytes, activation of the inflammasome and oxidative changes to lipoproteins.

NET components such as MPO trigger macrophages to release ROS and other cytokines. $^{225}$ In turn, ROS can modify LDL to oxLDL, which drives foam cell formation. ${ }^{226,227}$ Released cytokines recruit immune cells and oxLDL can trigger neutrophils for further NETosis ${ }^{215}$, augmenting plaque inflammation and formation. MPO or NADPH oxidase can also lead to oxidative modifications of HDL and thereby reduces its beneficial cholesterol efflux capacity. ${ }^{228}$ Other NETs components like cathepsin G, cathelicidins and cathelicidin-related antimicrobial peptide (CRAMP) exhibit monocyte-chemotactic activity. ${ }^{229-232}$ CRAMP, together with self-DNA released by NETs or other dying cells, can trigger plasmacytoid dendritic cells (pDCs)-driven autoimmune activation ${ }^{233}$, which has been shown to aggravate atherosclerosis lesions. ${ }^{234}$ Recently, Warnatsch et al. has shown that NETs lead to inflammasome activation in macrophages. ${ }^{216}$ In this setting, cholesterol crystal-activated NETs present the $1^{\text {st }}$ signal for inflammasome activation in macrophages resulting in pre-mature interleukin (IL)-1 $\beta$, with cholesterol crystals being the $2^{\text {nd }}$ signal, leading to 
mature IL-1 $\beta .^{216}$ Subsequently, IL-1 $\beta$ upregulates IL-17, which, in turn, enhances recruitment of immune cells to atherosclerotic site. Inflammasome activation itself can also lead to NET formation, which was shown by a study from Westerterp et al. using mice deficient for myeloid Abca1/g1 (ATP-binding cassette A1/G1), two key transporters in promoting cellular cholesterol efflux. Thereby intracellular cholesterol accumulation in myeloid cells induced NLRP3 inflammasome activation, which promoted neutrophil recruitment and NETs formation in atherosclerotic plaques and accelerated atherogenesis. ${ }^{222}$

\section{NETosis and diabetes-induced atherosclerosis}

Plasma NETs markers are not only correlated with severity of CAD, but also with severity of T2DM as judged by correlation of nucleosomes with HbA1c (hemoglobin A1c) in humans. ${ }^{235}$ Hyperglycemia seems to facilitate constitutively upregulated NETosis by priming neutrophils for NET formation ${ }^{210}$, and by providing increased substrate for NETosis, which has been shown to require glucose $\mathrm{e}^{236}$. The activation of neutrophils can be reversed by exposing neutrophils to physiological conditions. ${ }^{235,237}$ A study by Wong et al. showed that NETs impair wound healing in diabetic mice ${ }^{210}$ and humans ${ }^{238}$ and a recent study by our lab showed that diabetic mice have increased amount of NETs in atherosclerotic plaques. ${ }^{44}$ Given that a diabetic environment enhances processes that are related with NETosis, increased NET formation in atherosclerotic lesions from diabetic mice and the fact the wound healing resembles atherosclerosis regression in terms of the need of inflammation resolution, it is likely that NETs contribute to diabetes-impaired atherosclerosis regression and the increased CVD risk in patients suffering from diabetes.

\section{NETs as a therapeutic target}

Several possible therapeutic targets to reduce NETs have been reported. ${ }^{185}$ Two targets have been studied in context of atherosclerosis - 1.PAD4 inhibition and 2. DNase1. PAD4 inhibition prevents NET formation via the PAD4-mediated conversion of arginine to citrulline, thus inhibiting histone citrullination and chromatin decondensation. A study in $\mathrm{ApoE}^{-/-}$mice with $\mathrm{Pad}^{-/-}$myeloid cells showed diminished 
plaque formation with reduced NETosis and atherogenic inflammation. ${ }^{223}$ Studies using Clamidine, a pan-PAD inhibitor, have shown to reduce atherosclerosis in $A p o E^{-/-}$ mice by preventing NETosis and reducing the recruitment of neutrophils and monocytes. ${ }^{224}$ Further, in diabetic mice, Pad4 ${ }^{-/}$improved wound healing. ${ }^{210}$ In contrast, a study in Ldlr/ mice with Pad4-deficient bone marrow cells showed no altered atherogenesis. ${ }^{239}$ Further, Clamidine is not specific for PAD4 and targets all PAD isotypes, and additionally, PADs, including PAD4, have many functions such as the activation of dendritic ${ }^{240}$ and smooth muscle cells ${ }^{241}$ and cannot undoubtedly demonstrate NET involvement. A recent study by Lewis et al. reported specific PAD4 inhibition, which was sufficient to disrupt NET formation in mice and humans ${ }^{242}$ and further studies with this molecule are needed.

One of the major postulated side effects in inhibiting NETs formation via PAD4 is the reduced ability to fight infections. ${ }^{243}$ However, another study in PAD4-deficient mice did not show differences in morbidity compared to WT mice. ${ }^{244}$ Patients suffering from the Papillon-Lefèvre Syndrome (unable to produce NE) do not exhibit severe immunodeficiencies ${ }^{245,246}$, but suffer from periodontitis due to lack or deficiency of NETs. ${ }^{247}$ Therefore, NETs ability to fight pathogens might depend on pathogen type and location and further, other anti-microbial defense mechanisms might be able to compensate for NETs deficiency.

Given that that DNase1 (Pulmozyme ${ }^{\circledR}$ ) is clinically used for patients with cystic fibrosis ${ }^{248}$, where it has beneficial effects and shows no toxicity, DNase1 might be a better therapeutic approach than PAD4 inhibition. DNase1 is an endonuclease that clears NETs ${ }^{188,249}$ rather than blocking NET formation. Administering DNAse1 has been shown to lower plaque area in $A p o E^{-/}$mice ${ }^{216,250}$ and improve diabetes-impaired wound healing. ${ }^{210}$ Mutations in DNase1 activity has been shown in autoimmunity, in which increased NET formation is associated with disease severity. ${ }^{251,252}$ Since administering DNase1, thus increasing amount of active DNase, can overcome deleterious effects of NETs, e.g. improve wound healing despite ongoing hyperglycemia, it suggests that DNase1 activity might also be decreased in diabetes. However, one study shows that DNase 1 activity is actually increased in diabetes. ${ }^{253}$ Genetic deletion of DNase1 did not increase NET formation in vitro, while double KO of DNase1 and DNase1L3 did. ${ }^{254}$ Further, only double KO mice with chronic neutrophilia developed vascular occlusion in line with increased NET formation, while 
expression of either endonuclease subtype was able to prevent it. This indicates that other endonucleases like DNase1L3 are also able to degrade NETs. Further, although DNase1 has been shown to efficiently remove dsDNA, it has little effect on other NET components, such as NE, that might continue to cause inflammation at the vessel wall. ${ }^{255}$ This is in line with a study in $A p o E^{-/-}$mice, which showed no effect of DNase1 administration on lesion size after 4 months of high-fat diet. ${ }^{256}$ Further studies in the implication of DNase1 in atherosclerosis and diabetes are needed, including studies to evaluate different endonuclease types.

\section{Knowledge Gap}

Individuals suffering from diabetes have an increased CVD risk despite lipid-lowering therapy. Two of the postulated reasons are diabetic dyslipidemia and hyperglycemia. The connection between low HDL-C and elevated TG with CVDs risk has been shown. Due to failure of beneficial CVD outcomes from HDL-C raising trials, assessment of other HDL functions such as CEC has become of interest. However, studies on how these HDL characteristics in diabetes associate with CVD are scant. The close relationship of HyperTG and HDL-C has been described, but how HyperTG affects other HDL characteristics than HDL-C is unclear. Although evidence is increasing that HyperTG is the causal reason for increased CVD risk, current clinical trials showing CVD benefits by lowering TG levels are still inconclusive and data from pre-clinical trials are missing.

Hyperglycemia is known as an independent risk factor for CVD. One hypothesis is that this is due to stimulation of myelopoiesis. Activation of the bone marrow is thought to increase circulating levels of monocytes and neutrophils. While the role of monocytederived macrophages is well established, less is known about neutrophils. Recent studies have described neutrophils ability to undergo a process called NETosis that leads to the formation of pro-inflammatory NETs. How NETs behave during atherosclerosis regression, especially in a diabetic state in which regression is impaired, has not been established yet. 


\section{Outline}

In this Thesis, we have evaluated the effect of two hallmarks of diabetes in atherosclerosis regression (Figure 4). In Part1, I am discussing the role of diabetic dyslipidemia, namely low HDL-C and HyperTG, on atherosclerosis regression. In fact, my research focused on HDL characteristics other than HDL-C and their associations with CVD risk in a human population. In experimental mouse studies, I have studied the effect of HyperTG on some of these HDL characteristics as well as its impact on atherosclerosis regression. In Part2, I describe the effect of hyperglycemia-induced neutrophilia on the formation of NETs in atherosclerotic plaques.

Chapter 2 describes the associations of different HDL parameters with prevalent atherosclerosis and CVD/CVE in a total population as well as in subjects with prediabetes and T2DM in a human cohort. Chapter 3 introduces the mouse model used to create mice with HyperTG and low HDL-C, i.e. the lipoprotein lipase (LpL) deficient mice, and describes the role of $\mathrm{LpL}$ in vitro and in vivo. Next, in Chapter 4, I investigated the effect of HyperTG due to LpL deficiency on HDL with and without hCETP expression, and in turn on atherosclerosis regression in mice and people. Chapter 5 shows the role of NET formation in diabetes-impaired atherosclerosis regression in diabetic mice and in Chapter 6, I discuss different endonucleases, known to degrade NETs, and their involvement in atherosclerosis pro-and regression. In chapter $\mathbf{7}$, I provide a summary and conclusion on the findings of this thesis, including their impact for CVD. 
Chapter 1

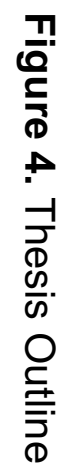

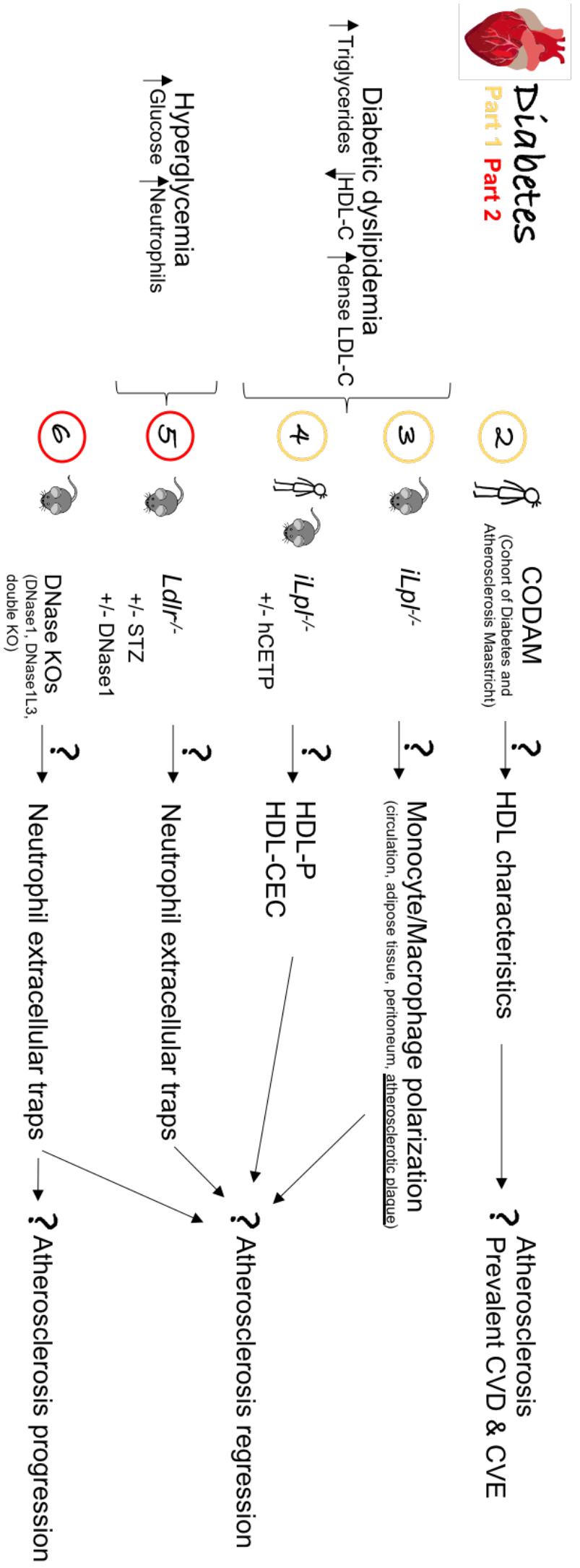




\section{References}

1 Global Report on Diabetes. World Health Organization, 2016

2 Dall, T. M. et al. The Economic Burden of Elevated Blood Glucose Levels in 2017: Diagnosed and Undiagnosed Diabetes, Gestational Diabetes Mellitus, and Prediabetes. Diabetes Care 42, 1661-1668, doi:10.2337/dc18-1226 (2019).

3 Federation, I. D. Diabetes facts \& figures, $<$ https://www.idf.org/aboutdiabetes/what-is-diabetes/facts-figures.html> (2017).

$4 \quad$ National Diabetes Statistics report. (Center for Disease Control and Prevention, 2017).

5 Low Wang, C. C., Hess, C. N., Hiatt, W. R. \& Goldfine, A. B. Clinical Update: Cardiovascular Disease in Diabetes Mellitus: Atherosclerotic Cardiovascular Disease and Heart Failure in Type 2 Diabetes Mellitus - Mechanisms, Management, and Clinical Considerations. Circulation 133, 2459-2502, doi:10.1161/CIRCULATIONAHA.116.022194 (2016).

6 Dhindsa, D. S., Sandesara, P. B. \& Shapiro, M. D. The Intersection of Diabetes and Cardiovascular Disease-A Focus on New Therapies. Front Cardiovasc Med 5, 160, doi:10.3389/fcvm.2018.00160 (2018).

7 Nabel, E. G. \& Braunwald, E. A tale of coronary artery disease and myocardial infarction. N Engl J Med 366, 54-63, doi:10.1056/NEJMra1112570 (2012).

8 Bloom, D. E., Cafiero, E.T., Jané-Llopis, E., Abrahams-Gessel, S., Bloom, L.R., Fathima, S., Feigl, A.B., Gaziano, T., Mowafi, M., Pandya, A., Prettner, K., Rosenberg, L., Seligman, B., Stein, A., \& Weinstein, C. The Global Economic Burden of Non-communicable Diseases, <http://apps.who.int/medicinedocs/documents/s18806en/s18806en.pdf> (2011).

9 Ross, R. Atherosclerosis--an inflammatory disease. N Engl J Med 340, 115126, doi:10.1056/NEJM199901143400207 (1999).

10 McGill, H. C., Jr. et al. Origin of atherosclerosis in childhood and adolescence. Am J Clin Nutr 72, 1307S-1315S, doi:10.1093/ajcn/72.5.1307s (2000).

11 Tabas, I., Williams, K. J. \& Borén, J. in Circulation Vol. 116 1832-1844 (2007).

12 Swirski, F. K. \& Nahrendorf, M. Vol. 86 161-166 (2013).

13 Falk, E., Nakano, M., Bentzon, J. F., Finn, A. V. \& Virmani, R. Update on acute coronary syndromes: the pathologists' view. Eur Heart J 34, 719-728, doi:10.1093/eurheartj/ehs411 (2013).

14 Bourantas, C. V., Garcia-Garcia, H. M., Diletti, R., Muramatsu, T. \& Serruys, P. W. Early detection and invasive passivation of future culprit lesions: a future potential or an unrealistic pursuit of chimeras? Am Heart J 165, 869-881 e864, doi:10.1016/j.ahj.2013.02.015 (2013).

15 Williams, K. J., Feig, J. E. \& Fisher, E. A. Rapid regression of atherosclerosis: insights from the clinical and experimental literature. Nat Clin Pract Cardiovasc Med 5, 91-102, doi:10.1038/ncpcardio1086 (2008).

16 Vartiainen, I. \& Kanerva, K. Arteriosclerosis and war-time. Ann Med Intern Fenn 36, 748-758 (1947).

17 Wilens, S. L. The Resorption of Arterial Atheromatous Deposits in Wasting Disease. Am J Pathol 23, 793-804 (1947). 
18 Wanscher, O., Clemmesen, J. \& Nielsen, A. Negative correlation between atherosclerosis and carcinoma. Br J Cancer 5, 172-174, doi:10.1038/bjc. 1951.18 (1951).

19 Nicholls, S. J. et al. Effect of Evolocumab on Progression of Coronary Disease in Statin-Treated Patients: The GLAGOV Randomized Clinical Trial. JAMA 316, 2373-2384, doi:10.1001/jama.2016.16951 (2016).

20 Zhao, X. Q. et al. Effects of combination lipid therapy on coronary stenosis progression and clinical cardiovascular events in coronary disease patients with metabolic syndrome: a combined analysis of the Familial Atherosclerosis Treatment Study (FATS), the HDL-Atherosclerosis Treatment Study (HATS), and the Armed Forces Regression Study (AFREGS). Am J Cardiol 104, $1457-$ 1464, doi:10.1016/j.amjcard.2009.07.035 (2009).

21 Nissen, S. E. et al. Effect of intensive compared with moderate lipid-lowering therapy on progression of coronary atherosclerosis: a randomized controlled trial. JAMA 291, 1071-1080, doi:10.1001/jama.291.9.1071 (2004).

22 Nissen, S. E. et al. Effect of very high-intensity statin therapy on regression of coronary atherosclerosis: the ASTEROID trial. JAMA 295, 1556-1565, doi:10.1001/jama.295.13.jpc60002 (2006).

23 Stein, Y. \& Stein, O. Does therapeutic intervention achieve slowing of progression or bona fide regression of atherosclerotic lesions? Arterioscler Thromb Vasc Biol 21, 183-188, doi:10.1161/01.atv.21.2.183 (2001).

24 Brown, B. G., Zhao, X. Q., Sacco, D. E. \& Albers, J. J. Lipid lowering and plaque regression. New insights into prevention of plaque disruption and clinical events in coronary disease. Circulation 87, 1781-1791, doi:10.1161/01.cir.87.6.1781 (1993).

25 Nicholls, S. J. et al. Effect of Evolocumab on Coronary Plaque Composition. J Am Coll Cardiol 72, 2012-2021, doi:10.1016/j.jacc.2018.06.078 (2018).

26 Tarkin, J. M. et al. Imaging Atherosclerosis. Circ Res 118, 750-769, doi:10.1161/CIRCRESAHA.115.306247 (2016).

27 Patel, K. et al. Invasive or non-invasive imaging for detecting high-risk coronary lesions? Expert Rev Cardiovasc Ther 15, 165-179, doi:10.1080/14779072.2017.1297231 (2017).

28 Burke, A. C. \& Huff, M. W. Regression of atherosclerosis: lessons learned from genetically modified mouse models. Curr Opin Lipidol 29, 87-94, doi:10.1097/MOL.0000000000000493 (2018).

29 Barrett, T. J. Macrophages in Atherosclerosis Regression. Arterioscler Thromb Vasc Biol, ATVBAHA119312802, doi:10.1161/ATVBAHA.119.312802 (2019).

30 Rahman, K. \& Fisher, E. A. Insights From Pre-Clinical and Clinical Studies on the Role of Innate Inflammation in Atherosclerosis Regression. Front Cardiovasc Med 5, 32, doi:10.3389/fcvm.2018.00032 (2018).

31 Plump, A. S. et al. Severe hypercholesterolemia and atherosclerosis in apolipoprotein E-deficient mice created by homologous recombination in ES cells. Cell 71, 343-353, doi:10.1016/0092-8674(92)90362-g (1992).

32 Zhang, S. H., Reddick, R. L., Piedrahita, J. A. \& Maeda, N. Spontaneous hypercholesterolemia and arterial lesions in mice lacking apolipoprotein $\mathrm{E}$. Science 258, 468-471, doi:10.1126/science.1411543 (1992).

33 Ishibashi, S. et al. Hypercholesterolemia in low density lipoprotein receptor knockout mice and its reversal by adenovirus-mediated gene delivery. J Clin Invest 92, 883-893, doi:10.1172/JCI116663 (1993). 
34 van den Maagdenberg, A. M. et al. Transgenic mice carrying the apolipoprotein E3-Leiden gene exhibit hyperlipoproteinemia. J Biol Chem 268, 10540-10545 (1993).

35 Distel, E. et al. miR33 inhibition overcomes deleterious effects of diabetes mellitus on atherosclerosis plaque regression in mice. Circ Res 115, 759-769, doi:10.1161/CIRCRESAHA.115.304164 (2014).

36 Stevenson, S. C. et al. Phenotypic correction of hypercholesterolemia in apoEdeficient mice by adenovirus-mediated in vivo gene transfer. Arterioscler Thromb Vasc Biol 15, 479-484, doi:10.1161/01.atv.15.4.479 (1995).

37 Tsukamoto, K., Tangirala, R., Chun, S. H., Pure, E. \& Rader, D. J. Rapid regression of atherosclerosis induced by liver-directed gene transfer of ApoE in ApoE-deficient mice. Arterioscler Thromb Vasc Biol 19, 2162-2170, doi:10.1161/01.atv.19.9.2162 (1999).

38 Reis, E. D. et al. Dramatic remodeling of advanced atherosclerotic plaques of the apolipoprotein E-deficient mouse in a novel transplantation model. J Vasc Surg 34, 541-547, doi:10.1067/mva.2001.115963 (2001).

39 Feig, J. E. et al. Reversal of hyperlipidemia with a genetic switch favorably affects the content and inflammatory state of macrophages in atherosclerotic plaques. Circulation 123, 989-998, doi:10.1161/CIRCULATIONAHA.110.984146 (2011).

40 Nagareddy, P. R. et al. Hyperglycemia promotes myelopoiesis and impairs the resolution of atherosclerosis. Cell Metab 17, 695-708, doi:10.1016/j.cmet.2013.04.001 (2013).

41 Peled, M. et al. A wild-type mouse-based model for the regression of inflammation in atherosclerosis. PLoS One 12, e0173975, doi:10.1371/journal.pone.0173975 (2017).

42 Basu, D. et al. Novel Reversible Model of Atherosclerosis and Regression Using Oligonucleotide Regulation of the LDL Receptor. Circ Res 122, 560567, doi:10.1161/CIRCRESAHA.117.311361 (2018).

43 Bartels, E. D., Christoffersen, C., Lindholm, M. W. \& Nielsen, L. B. Altered metabolism of LDL in the arterial wall precedes atherosclerosis regression. Circ Res 117, 933-942, doi:10.1161/CIRCRESAHA.115.307182 (2015).

44 Barrett, T. J. et al. Apolipoprotein AI) Promotes Atherosclerosis Regression in Diabetic Mice by Suppressing Myelopoiesis and Plaque Inflammation. Circulation 140, 1170-1184, doi:10.1161/CIRCULATIONAHA.119.039476 (2019).

45 Hewing, B. et al. Effects of native and myeloperoxidase-modified apolipoprotein a-I on reverse cholesterol transport and atherosclerosis in mice. Arterioscler Thromb Vasc Biol 34, 779-789, doi:10.1161/ATVBAHA.113.303044 (2014).

46 Yuan, C. et al. Human Aldose Reductase Expression Prevents Atherosclerosis Regression in Diabetic Mice. Diabetes 67, 1880-1891, doi:10.2337/db18-0156 (2018).

$47 \mathrm{Li}, \mathrm{J}$. et al. Secreted PCSK9 promotes LDL receptor degradation independently of proteolytic activity. Biochem J 406, 203-207, doi:10.1042/BJ20070664 (2007).

48 Goldstein, J. L., Hazzard, W. R., Schrott, H. G., Bierman, E. L. \& Motulsky, A. G. Hyperlipidemia in coronary heart disease. I. Lipid levels in 500 survivors of 
myocardial infarction. J Clin Invest 52, 1533-1543, doi:10.1172/JCl107331 (1973).

49 Nordestgaard, B. G. Triglyceride-Rich Lipoproteins and Atherosclerotic Cardiovascular Disease: New Insights From Epidemiology, Genetics, and Biology. Circ Res 118, 547-563, doi:10.1161/CIRCRESAHA.115.306249 (2016).

50 Ridker, P. M., Pradhan, A., MacFadyen, J. G., Libby, P. \& Glynn, R. J. Cardiovascular benefits and diabetes risks of statin therapy in primary prevention: an analysis from the JUPITER trial. Lancet 380, 565-571, doi:10.1016/S0140-6736(12)61190-8 (2012).

51 Murphy, S. A., Cannon, C. P., Wiviott, S. D., McCabe, C. H. \& Braunwald, E. Reduction in recurrent cardiovascular events with intensive lipid-lowering statin therapy compared with moderate lipid-lowering statin therapy after acute coronary syndromes from the PROVE IT-TIMI 22 (Pravastatin or Atorvastatin Evaluation and Infection Therapy-Thrombolysis In Myocardial Infarction 22) trial. J Am Coll Cardiol 54, 2358-2362, doi:10.1016/j.jacc.2009.10.005 (2009).

52 Benjamin, E. J. et al. Heart Disease and Stroke Statistics-2017 Update: A Report From the American Heart Association. Circulation 135, e146-e603, doi:10.1161/CIR.0000000000000485 (2017).

53 Pacini, F. Best practice \& research clinical endocrinology \& metabolism. Thyroid nodules and cancer. Preface. Best Pract Res Clin Endocrinol Metab 22, vii, doi:10.1016/j.beem.2008.11.001 (2008).

54 Nicholls, S. J. et al. Effect of diabetes on progression of coronary atherosclerosis and arterial remodeling: a pooled analysis of 5 intravascular ultrasound trials. J Am Coll Cardiol 52, 255-262, doi:10.1016/j.jacc.2008.03.051 (2008).

55 Wu, L. \& Parhofer, K. G. Diabetic dyslipidemia. Metabolism 63, 1469-1479, doi:10.1016/j.metabol.2014.08.010 (2014).

56 Reiner, Z. Hypertriglyceridaemia and risk of coronary artery disease. Nat Rev Cardiol 14, 401-411, doi:10.1038/nrcardio.2017.31 (2017).

57 Jacobson, T. A. et al. National Lipid Association recommendations for patientcentered management of dyslipidemia: part 1 - executive summary. J Clin Lipido/ 8, 473-488, doi:10.1016/j.jacl.2014.07.007 (2014).

58 Carroll, M. D., Kit, B. K., Lacher, D. A., Shero, S. T. \& Mussolino, M. E. Trends in lipids and lipoproteins in US adults, 1988-2010. JAMA 308, 1545-1554, doi:10.1001/jama.2012.13260 (2012).

59 Nordestgaard, B. G. et al. Fasting is not routinely required for determination of a lipid profile: clinical and laboratory implications including flagging at desirable concentration cut-points-a joint consensus statement from the European Atherosclerosis Society and European Federation of Clinical Chemistry and Laboratory Medicine. Eur Heart J 37, 1944-1958, doi:10.1093/eurheartj/ehw152 (2016).

60 Xiao, C., Dash, S., Morgantini, C., Hegele, R. A. \& Lewis, G. F. Pharmacological Targeting of the Atherogenic Dyslipidemia Complex: The Next Frontier in CVD Prevention Beyond Lowering LDL Cholesterol. Diabetes 65, 1767-1778, doi:10.2337/db16-0046 (2016).

61 Dugi, K. A. et al. In vivo evidence for both lipolytic and nonlipolytic function of hepatic lipase in the metabolism of HDL. Arterioscler Thromb Vasc Biol 20, 793-800, doi:10.1161/01.atv.20.3.793 (2000). 
62 Maugeais, C. et al. Dose-dependent acceleration of high-density lipoprotein catabolism by endothelial lipase. Circulation 108, 2121-2126, doi:10.1161/01.CIR.0000092889.24713.DC (2003).

63 Brahm, A. J. \& Hegele, R. A. Chylomicronaemia--current diagnosis and future therapies. Nat Rev Endocrinol 11, 352-362, doi:10.1038/nrendo.2015.26 (2015).

64 Hegele, R. A. et al. The polygenic nature of hypertriglyceridaemia: implications for definition, diagnosis, and management. Lancet Diabetes Endocrinol 2, 655666, doi:10.1016/S2213-8587(13)70191-8 (2014).

65 Brahm, A. \& Hegele, R. A. Hypertriglyceridemia. Nutrients 5, 981-1001, doi:10.3390/nu5030981 (2013).

66 Investigators, A.-H. et al. Niacin in patients with low HDL cholesterol levels receiving intensive statin therapy. N Engl J Med 365, 2255-2267, doi:10.1056/NEJMoa1107579 (2011).

67 Baigent, C. et al. Efficacy and safety of cholesterol-lowering treatment: prospective meta-analysis of data from 90,056 participants in 14 randomised trials of statins. Lancet 366, 1267-1278, doi:10.1016/S0140-6736(05)67394-1 (2005).

68 Castelli, W. P. The triglyceride issue: a view from Framingham. Am Heart J 112, 432-437, doi:10.1016/0002-8703(86)90296-6 (1986).

69 Rhoads, G. G., Blackwelder, W. C., Stemmermann, G. N., Hayashi, T. \& Kagan, A. Coronary risk factors and autopsy findings in Japanese-American men. Lab Invest 38, 304-311 (1978).

70 Yano, K., Rhoads, G. G., Kagan, A. \& Tillotson, J. Dietary intake and the risk of coronary heart disease in Japanese men living in Hawaii. Am J Clin Nutr 31, 1270-1279, doi:10.1093/ajcn/31.7.1270 (1978).

71 Robertson, T. L. et al. Epidemiologic studies of coronary heart disease and stroke in Japanese men living in Japan, Hawaii and California. Coronary heart disease risk factors in Japan and Hawaii. Am J Cardiol 39, 244-249, doi:10.1016/s0002-9149(77)80198-7 (1977).

72 Emerging Risk Factors, C. et al. Major lipids, apolipoproteins, and risk of vascular disease. JAMA 302, 1993-2000, doi:10.1001/jama.2009.1619 (2009).

73 Miller, M. et al. Triglycerides and cardiovascular disease: a scientific statement from the American Heart Association. Circulation 123, 2292-2333, doi:10.1161/CIR.0b013e3182160726 (2011).

74 Jacobs, D. R., Jr. \& Barrett-Connor, E. Retest reliability of plasma cholesterol and triglyceride. The Lipid Research Clinics Prevalence Study. Am J Epidemiol 116, 878-885, doi:10.1093/oxfordjournals.aje.a113490 (1982).

75 NIH Consensus conference. Triglyceride, high-density lipoprotein, and coronary heart disease. NIH Consensus Development Panel on Triglyceride, High-Density Lipoprotein, and Coronary Heart Disease. JAMA 269, 505-510 (1993).

76 Pejic, R. N. \& Lee, D. T. Hypertriglyceridemia. J Am Board Fam Med 19, 310316 (2006).

77 Talayero, B. G. \& Sacks, F. M. The role of triglycerides in atherosclerosis. Curr Cardiol Rep 13, 544-552, doi:10.1007/s11886-011-0220-3 (2011).

78 Katcher, H. I., Hill, A. M., Lanford, J. L., Yoo, J. S. \& Kris-Etherton, P. M. Lifestyle approaches and dietary strategies to lower LDL-cholesterol and 
triglycerides and raise HDL-cholesterol. Endocrinol Metab Clin North Am 38, 45-78, doi:10.1016/j.ecl.2008.11.010 (2009).

79 Castelli, W. P. et al. HDL cholesterol and other lipids in coronary heart disease. The cooperative lipoprotein phenotyping study. Circulation 55, 767772, doi:10.1161/01.cir.55.5.767 (1977).

80 Gordon, D. J. \& Rifkind, B. M. High-density lipoprotein--the clinical implications of recent studies. N Engl J Med 321, 1311-1316, doi:10.1056/NEJM198911093211907 (1989).

81 Franceschini, G. Epidemiologic evidence for high-density lipoprotein cholesterol as a risk factor for coronary artery disease. Am J Cardiol 88, 9N$13 \mathrm{~N}$, doi:10.1016/s0002-9149(01)02146-4 (2001).

82 Nordestgaard, B. G. \& Varbo, A. Triglycerides and cardiovascular disease. Lancet 384, 626-635, doi:10.1016/S0140-6736(14)61177-6 (2014).

83 Austin, M. A., Hokanson, J. E. \& Edwards, K. L. Hypertriglyceridemia as a cardiovascular risk factor. Am J Cardiol 81, 7B-12B, doi:10.1016/s00029149(98)00031-9 (1998).

84 Patel, A. et al. Serum triglycerides as a risk factor for cardiovascular diseases in the Asia-Pacific region. Circulation 110, 2678-2686, doi:10.1161/01.CIR.0000145615.33955.83 (2004).

85 Sarwar, N. et al. Triglycerides and the risk of coronary heart disease: 10,158 incident cases among 262,525 participants in 29 Western prospective studies. Circulation 115, 450-458, doi:10.1161/CIRCULATIONAHA.106.637793 (2007).

86 Hokanson, J. E. \& Austin, M. A. Plasma triglyceride level is a risk factor for cardiovascular disease independent of high-density lipoprotein cholesterol level: a meta-analysis of population-based prospective studies. J Cardiovasc Risk 3, 213-219 (1996).

87 Brunner, D., Altman, S., Loebl, K., Schwartz, S. \& Levin, S. Serum cholesterol and triglycerides in patients suffering from ischemic heart disease and in healthy subjects. Atherosclerosis 28, 197-204, doi:10.1016/00219150(77)90157-5 (1977).

88 Fager, G., Wiklund, O., Olofsson, S. O., Wilhelmsen, L. \& Bondjers, G. Multivariate analyses of serum apolipoproteins and risk factors in relation to acute myocardial infarction. Arteriosclerosis 1, 273-279 (1981).

89 Gotto, A. M. et al. Relationship between plasma lipid concentrations and coronary artery disease in 496 patients. Circulation 56, 875-883, doi:10.1161/01.cir.56.5.875 (1977).

90 Hamsten, A., Walldius, G., Dahlen, G., Johansson, B. \& De Faire, U. Serum lipoproteins and apolipoproteins in young male survivors of myocardial infarction. Atherosclerosis 59, 223-235, doi:10.1016/0021-9150(86)90051-1 (1986).

91 Kukita, H., Imamura, Y., Hamada, M., Joh, T. \& Kokubu, T. Plasma lipids and lipoproteins in Japanese male patients with coronary artery disease and in their relatives. Atherosclerosis 42, 21-29, doi:10.1016/0021-9150(82)90122-8 (1982).

92 Scott, D. W., Gotto, A. M., Cole, J. S. \& Gorry, G. A. Plasma lipids as collateral risk factors in coronary artery disease--a study of 371 males with chest pain. $J$ Chronic Dis 31, 337-345, doi:10.1016/0021-9681(78)90049-8 (1978). 
93 Varbo, A. et al. Remnant cholesterol as a causal risk factor for ischemic heart disease. J Am Coll Cardiol 61, 427-436, doi:10.1016/j.jacc.2012.08.1026 (2013).

94 Do, R. et al. Common variants associated with plasma triglycerides and risk for coronary artery disease. Nat Genet 45, 1345-1352, doi:10.1038/ng.2795 (2013).

95 Thomsen, M., Varbo, A., Tybjaerg-Hansen, A. \& Nordestgaard, B. G. Low nonfasting triglycerides and reduced all-cause mortality: a mendelian randomization study. Clin Chem 60, 737-746, doi:10.1373/clinchem.2013.219881 (2014).

96 Nordestgaard, B. G., Benn, M., Schnohr, P. \& Tybjaerg-Hansen, A. Nonfasting triglycerides and risk of myocardial infarction, ischemic heart disease, and death in men and women. JAMA 298, 299-308, doi:10.1001/jama.298.3.299 (2007).

97 Freiberg, J. J., Tybjaerg-Hansen, A., Jensen, J. S. \& Nordestgaard, B. G. Nonfasting triglycerides and risk of ischemic stroke in the general population. JAMA 300, 2142-2152, doi:10.1001/jama.2008.621 (2008).

98 Murad, M. H. et al. The association of hypertriglyceridemia with cardiovascular events and pancreatitis: a systematic review and meta-analysis. BMC Endocr Disord 12, 2, doi:10.1186/1472-6823-12-2 (2012).

99 Avogaro, A. et al. Incidence of coronary heart disease in type 2 diabetic men and women: impact of microvascular complications, treatment, and geographic location. Diabetes Care 30, 1241-1247, doi:10.2337/dc06-2558 (2007).

100 Giorda, C. B. et al. Recurrence of cardiovascular events in patients with type 2 diabetes: epidemiology and risk factors. Diabetes Care 31, 2154-2159, doi:10.2337/dc08-1013 (2008).

101 Schulze, M. B. et al. Joint role of non-HDL cholesterol and glycated haemoglobin in predicting future coronary heart disease events among women with type 2 diabetes. Diabetologia 47, 2129-2136, doi:10.1007/s00125-0041593-2 (2004).

$102 \mathrm{Lu}, \mathrm{W}$. et al. Non-HDL cholesterol as a predictor of cardiovascular disease in type 2 diabetes: the strong heart study. Diabetes Care 26, 16-23, doi:10.2337/diacare.26.1.16 (2003).

103 Nilsson, P. M., Engstrom, G. \& Hedblad, B. The metabolic syndrome and incidence of cardiovascular disease in non-diabetic subjects--a populationbased study comparing three different definitions. Diabet Med 24, 464-472, doi:10.1111/j.1464-5491.2007.02142.x (2007).

104 Kadota, A. et al. Relationship between metabolic risk factor clustering and cardiovascular mortality stratified by high blood glucose and obesity: NIPPON DATA90, 1990-2000. Diabetes Care 30, 1533-1538, doi:10.2337/dc06-2074 (2007).

105 Ninomiya, J. K. et al. Association of the metabolic syndrome with history of myocardial infarction and stroke in the Third National Health and Nutrition Examination Survey. Circulation 109, 42-46, doi:10.1161/01.CIR.0000108926.04022.0C (2004).

106 Onat, A. et al. Plasma triglycerides, an independent predictor of cardiovascular disease in men: a prospective study based on a population 
with prevalent metabolic syndrome. Int J Cardiol 108, 89-95, doi:10.1016/j.ijcard.2005.06.056 (2006).

107 Austin, M. A. et al. Cardiovascular disease mortality in familial forms of hypertriglyceridemia: A 20-year prospective study. Circulation 101, 2777-2782, doi:10.1161/01.cir.101.24.2777 (2000).

108 Hopkins, P. N. et al. Coronary artery disease risk in familial combined hyperlipidemia and familial hypertriglyceridemia: a case-control comparison from the National Heart, Lung, and Blood Institute Family Heart Study. Circulation 108, 519-523, doi:10.1161/01.CIR.0000081777.17879.85 (2003).

109 Musunuru, K. \& Kathiresan, S. Surprises From Genetic Analyses of Lipid Risk Factors for Atherosclerosis. Circ Res 118, 579-585, doi:10.1161/CIRCRESAHA.115.306398 (2016).

110 Teslovich, T. M. et al. Biological, clinical and population relevance of 95 loci for blood lipids. Nature 466, 707-713, doi:10.1038/nature09270 (2010).

111 Triglyceride Coronary Disease Genetics, C. et al. Triglyceride-mediated pathways and coronary disease: collaborative analysis of 101 studies. Lancet 375, 1634-1639, doi:10.1016/S0140-6736(10)60545-4 (2010).

112 Jorgensen, A. B., Frikke-Schmidt, R., Nordestgaard, B. G. \& TybjaergHansen, A. Loss-of-function mutations in APOC3 and risk of ischemic vascular disease. N Engl J Med 371, 32-41, doi:10.1056/NEJMoa1308027 (2014).

$113 \mathrm{Tg}$ et al. Loss-of-function mutations in APOC3, triglycerides, and coronary disease. N Engl J Med 371, 22-31, doi:10.1056/NEJMoa1307095 (2014).

114 Dewey, F. E. et al. Genetic and Pharmacologic Inactivation of ANGPTL3 and Cardiovascular Disease. N Engl J Med 377, 211-221, doi:10.1056/NEJMoa1612790 (2017).

115 Myocardial Infarction, G. et al. Coding Variation in ANGPTL4, LPL, and SVEP1 and the Risk of Coronary Disease. N Engl J Med 374, 1134-1144, doi:10.1056/NEJMoa1507652 (2016).

116 Nordestgaard, B. G., Stender, S. \& Kjeldsen, K. Severe hypertriglyceridemia, large lipoproteins and protection against atherosclerosis. Scand J Clin Lab Invest Supp/ 186, 7-12 (1987).

117 Nordestgaard, B. G., Tybjaerg-Hansen, A. \& Lewis, B. Influx in vivo of low density, intermediate density, and very low density lipoproteins into aortic intimas of genetically hyperlipidemic rabbits. Roles of plasma concentrations, extent of aortic lesion, and lipoprotein particle size as determinants.

Arterioscler Thromb 12, 6-18 (1992).

118 Nordestgaard, B. G. \& Zilversmit, D. B. Large lipoproteins are excluded from the arterial wall in diabetic cholesterol-fed rabbits. J Lipid Res 29, 1491-1500 (1988).

119 Rapp, J. H. et al. Triglyceride-rich lipoproteins isolated by selected-affinity antiapolipoprotein $B$ immunosorption from human atherosclerotic plaque. Arterioscler Thromb 14, 1767-1774 (1994).

120 Benlian, P. et al. Premature atherosclerosis in patients with familial chylomicronemia caused by mutations in the lipoprotein lipase gene. $N$ Engl J Med 335, 848-854, doi:10.1056/NEJM199609193351203 (1996).

121 Goldberg, I. J., Eckel, R. H. \& McPherson, R. Triglycerides and heart disease: still a hypothesis? Arterioscler Thromb Vasc Biol 31, 1716-1725, doi:10.1161/ATVBAHA.111.226100 (2011). 
122 Zilversmit, D. B. A proposal linking atherogenesis to the interaction of endothelial lipoprotein lipase with triglyceride-rich lipoproteins. Circ Res 33, 633-638, doi:10.1161/01.res.33.6.633 (1973).

123 Mead, J. R., Irvine, S. A. \& Ramji, D. P. Lipoprotein lipase: structure, function, regulation, and role in disease. $\mathrm{J} \mathrm{Mol} \mathrm{Med} \mathrm{(Berl)} \mathrm{80,} \mathrm{753-769,}$ doi:10.1007/s00109-002-0384-9 (2002).

124 Goldstein, J. L., Ho, Y. K., Brown, M. S., Innerarity, T. L. \& Mahley, R. W. Cholesteryl ester accumulation in macrophages resulting from receptormediated uptake and degradation of hypercholesterolemic canine beta-very low density lipoproteins. J Biol Chem 255, 1839-1848 (1980).

125 Takahashi, S. et al. The very low-density lipoprotein (VLDL) receptor: characterization and functions as a peripheral lipoprotein receptor. $J$ Atheroscler Thromb 11, 200-208, doi:10.5551/jat.11.200 (2004).

126 Rosenson, R. S., Davidson, M. H., Hirsh, B. J., Kathiresan, S. \& Gaudet, D. Genetics and causality of triglyceride-rich lipoproteins in atherosclerotic cardiovascular disease. J Am Coll Cardiol 64, 2525-2540, doi:10.1016/j.jacc.2014.09.042 (2014).

127 Alaupovic, P., Mack, W. J., Knight-Gibson, C. \& Hodis, H. N. The role of triglyceride-rich lipoprotein families in the progression of atherosclerotic lesions as determined by sequential coronary angiography from a controlled clinical trial. Arterioscler Thromb Vasc Biol 17, 715-722, doi:10.1161/01.atv.17.4.715 (1997).

128 Bhatt, D. \& Tannock, L. in Endotext (eds K. R. Feingold et al.) (2000).

129 Chapman, M. J. et al. Triglyceride-rich lipoproteins and high-density lipoprotein cholesterol in patients at high risk of cardiovascular disease: evidence and guidance for management. Eur Heart J 32, 1345-1361, doi:10.1093/eurheartj/ehr112 (2011).

130 Thorin, E. Vascular disease risk in patients with hypertriglyceridemia: endothelial progenitor cells, oxidative stress, accelerated senescence, and impaired vascular repair. Can J Cardiol 27, 538-540, doi:10.1016/j.cjca.2011.03.014 (2011).

131 Aung, H. H. et al. Induction of ATF3 gene network by triglyceride-rich lipoprotein lipolysis products increases vascular apoptosis and inflammation. Arterioscler Thromb Vasc Biol 33, 2088-2096, doi:10.1161/ATVBAHA.113.301375 (2013).

132 Preiss, D. et al. Lipid-modifying therapies and risk of pancreatitis: a metaanalysis. JAMA 308, 804-811, doi:10.1001/jama.2012.8439 (2012).

133 Murphy, M. J., Sheng, X., MacDonald, T. M. \& Wei, L. Hypertriglyceridemia and acute pancreatitis. JAMA Intern Med 173, 162-164, doi:10.1001/2013.jamainternmed.477 (2013).

134 Lindkvist, B., Appelros, S., Regner, S. \& Manjer, J. A prospective cohort study on risk of acute pancreatitis related to serum triglycerides, cholesterol and fasting glucose. Pancreatology 12, 317-324, doi:10.1016/j.pan.2012.05.002 (2012).

135 European Association for Cardiovascular, P. et al. ESC/EAS Guidelines for the management of dyslipidaemias: the Task Force for the management of dyslipidaemias of the European Society of Cardiology (ESC) and the European Atherosclerosis Society (EAS). Eur Heart J 32, 1769-1818, doi:10.1093/eurheartj/ehr158 (2011). 
136 Perk, J. et al. European Guidelines on cardiovascular disease prevention in clinical practice (version 2012). The Fifth Joint Task Force of the European Society of Cardiology and Other Societies on Cardiovascular Disease Prevention in Clinical Practice (constituted by representatives of nine societies and by invited experts). Eur Heart J 33, 1635-1701, doi:10.1093/eurheartj/ehs092 (2012).

137 National Cholesterol Education Program Expert Panel on Detection, E. \& Treatment of High Blood Cholesterol in, A. Third Report of the National Cholesterol Education Program (NCEP) Expert Panel on Detection, Evaluation, and Treatment of High Blood Cholesterol in Adults (Adult Treatment Panel III) final report. Circulation 106, 3143-3421 (2002).

138 Goff, D. C., Jr. et al. 2013 ACC/AHA guideline on the assessment of cardiovascular risk: a report of the American College of Cardiology/American Heart Association Task Force on Practice Guidelines. Circulation 129, S49-73, doi:10.1161/01.cir.0000437741.48606.98 (2014).

139 Berglund, L. et al. Evaluation and treatment of hypertriglyceridemia: an Endocrine Society clinical practice guideline. J Clin Endocrinol Metab 97, 2969-2989, doi:10.1210/jc.2011-3213 (2012).

140 Brunzell, J. D. et al. Lipoprotein management in patients with cardiometabolic risk: consensus conference report from the American Diabetes Association and the American College of Cardiology Foundation. J Am Coll Cardiol 51, 1512-1524, doi:10.1016/j.jacc.2008.02.034 (2008).

141 Watts, G. F., Ooi, E. M. \& Chan, D. C. Demystifying the management of hypertriglyceridaemia. Nat Rev Cardiol 10, 648-661, doi:10.1038/nrcardio.2013.140 (2013).

142 Rubins, H. B. et al. Gemfibrozil for the secondary prevention of coronary heart disease in men with low levels of high-density lipoprotein cholesterol. Veterans Affairs High-Density Lipoprotein Cholesterol Intervention Trial Study Group. N Engl J Med 341, 410-418, doi:10.1056/NEJM199908053410604 (1999).

143 Robins, S. J. et al. Relation of gemfibrozil treatment and lipid levels with major coronary events: VA-HIT: a randomized controlled trial. JAMA 285, 15851591, doi:10.1001/jama.285.12.1585 (2001).

144 Jun, M. et al. Effects of fibrates on cardiovascular outcomes: a systematic review and meta-analysis. Lancet 375, 1875-1884, doi:10.1016/S01406736(10)60656-3 (2010).

145 Barter, P. J. \& Rye, K. A. Is there a role for fibrates in the management of dyslipidemia in the metabolic syndrome? Arterioscler Thromb Vasc Biol 28, 39-46, doi:10.1161/ATVBAHA.107.148817 (2008).

146 Elam, M. B. et al. Association of Fenofibrate Therapy With Long-term Cardiovascular Risk in Statin-Treated Patients With Type 2 Diabetes. JAMA Cardiol 2, 370-380, doi:10.1001/jamacardio.2016.4828 (2017).

147 Branchi, A. et al. Lowering effects of four different statins on serum triglyceride level. Eur J Clin Pharmacol 55, 499-502, doi:10.1007/s002280050663 (1999).

148 Sacks, F. M. et al. Coronary heart disease in patients with low LDLcholesterol: benefit of pravastatin in diabetics and enhanced role for HDLcholesterol and triglycerides as risk factors. Circulation 105, 1424-1428, doi:10.1161/01.cir.0000012918.84068.43 (2002).

149 Group, A. S. et al. Effects of combination lipid therapy in type 2 diabetes mellitus. N Engl J Med 362, 1563-1574, doi:10.1056/NEJMoa1001282 (2010). 
150 Reyes-Soffer, G. et al. Effect of combination therapy with fenofibrate and simvastatin on postprandial lipemia in the ACCORD lipid trial. Diabetes Care 36, 422-428, doi:10.2337/dc11-2556 (2013).

151 Linton, M. R. F. et al. in Endotext (eds K. R. Feingold et al.) (2000).

152 Kamanna, V. S., Ganji, S. H. \& Kashyap, M. L. Recent advances in niacin and lipid metabolism. Curr Opin Lipidol 24, 239-245, doi:10.1097/MOL.0b013e3283613a68 (2013).

153 Feingold, K. R. \& Grunfeld, C. in Endotext (eds K. R. Feingold et al.) (2000).

154 Group, H.-T. C. in New England Journal of Medicine Vol. 371 203-212 (2014).

155 Guyton, J. R. et al. Relationship of lipoproteins to cardiovascular events: the AIM-HIGH Trial (Atherothrombosis Intervention in Metabolic Syndrome With Low HDL/High Triglycerides and Impact on Global Health Outcomes). J Am Coll Cardiol 62, 1580-1584, doi:10.1016/j.jacc.2013.07.023 (2013).

156 Rimm, E. B. et al. Seafood Long-Chain n-3 Polyunsaturated Fatty Acids and Cardiovascular Disease: A Science Advisory From the American Heart Association. Circulation 138, e35-e47, doi:10.1161/CIR.0000000000000574 (2018).

157 Mozaffarian, D. \& Wu, J. H. Omega-3 fatty acids and cardiovascular disease: effects on risk factors, molecular pathways, and clinical events. J Am Coll Cardiol 58, 2047-2067, doi:10.1016/j.jacc.2011.06.063 (2011).

158 Prasad, K. Flaxseed and cardiovascular health. J Cardiovasc Pharmacol 54, 369-377, doi:10.1097/FJC.0b013e3181af04e5 (2009).

159 Brenna, J. T. et al. alpha-Linolenic acid supplementation and conversion to n3 long-chain polyunsaturated fatty acids in humans. Prostaglandins Leukot Essent Fatty Acids 80, 85-91, doi:10.1016/j.plefa.2009.01.004 (2009).

160 Bhatt, D. L. et al. Effects of Icosapent Ethyl on Total Ischemic Events: From REDUCE-IT. J Am Coll Cardiol 73, 2791-2802, doi:10.1016/j.jacc.2019.02.032 (2019).

161 Bhatt, D. L. et al. Cardiovascular Risk Reduction with Icosapent Ethyl for Hypertriglyceridemia. N Engl J Med 380, 11-22, doi:10.1056/NEJMoa1812792 (2019).

162 Huet, F., Roubille, C. \& Roubille, F. Is hypertriglyceridemia atherogenic? Curr Opin Lipidol 30, 291-299, doi:10.1097/MOL.0000000000000622 (2019).

163 Chistiakov, D. A., Bobryshev, Y. V. \& Orekhov, A. N. Neutrophil's weapons in atherosclerosis. Exp Mol Pathol 99, 663-671, doi:10.1016/j.yexmp.2015.11.011 (2015).

164 Kondo, M. Lymphoid and myeloid lineage commitment in multipotent hematopoietic progenitors. Immunol Rev 238, 37-46, doi:10.1111/j.1600065X.2010.00963.x (2010).

165 Fearon, D. T. \& Locksley, R. M. The instructive role of innate immunity in the acquired immune response. Science $272,50-53$, doi:10.1126/science.272.5258.50 (1996).

166 Libby, P., Nahrendorf, M. \& Swirski, F. K. Leukocytes Link Local and Systemic Inflammation in Ischemic Cardiovascular Disease: An Expanded "Cardiovascular Continuum". J Am Coll Cardiol 67, 1091-1103, doi:10.1016/j.jacc.2015.12.048 (2016).

167 Hansson, G. K. Inflammation, atherosclerosis, and coronary artery disease. N Engl J Med 352, 1685-1695, doi:10.1056/NEJMra043430 (2005). 
168 Ridker, P. M. et al. Antiinflammatory Therapy with Canakinumab for Atherosclerotic Disease. N Engl J Med 377, 1119-1131, doi:10.1056/NEJMoa1707914 (2017).

169 Tardif, J. C. et al. Efficacy and Safety of Low-Dose Colchicine after Myocardial Infarction. N Engl J Med, doi:10.1056/NEJMoa1912388 (2019).

170 Keefer, C. S. \& Resnik, W. H. Vol. 6 769-807 (1928).

171 Hilgendorf, I. \& Swirski, F. K. Making a difference: monocyte heterogeneity in cardiovascular disease. Curr Atheroscler Rep 14, 450-459, doi:10.1007/s11883-012-0274-8 (2012).

172 Sweetnam, P. M., Thomas, H. F., Yarnell, J. W., Baker, I. A. \& Elwood, P. C. Total and differential leukocyte counts as predictors of ischemic heart disease: the Caerphilly and Speedwell studies. Am J Epidemiol 145, 416-421, doi:10.1093/oxfordjournals.aje.a009123 (1997).

173 Grau, A. J. et al. Leukocyte count as an independent predictor of recurrent ischemic events. Stroke 35, 1147-1152, doi:10.1161/01.STR.0000124122.71702.64 (2004).

174 Nikolajczyk, B. S., Jagannathan-Bogdan, M., Shin, H. \& Gyurko, R. State of the union between metabolism and the immune system in type 2 diabetes. Genes and Immunity 12, 239-250, doi:10.1038/gene.2011.14 (2011).

175 Alba-Loureiro, T. C. et al. Neutrophil function and metabolism in individuals with diabetes mellitus. Brazilian Journal of Medical and Biological Research 40, 1037-1044, doi:10.1590/S0100-879X2006005000143 (2007).

176 Megens, R. T. A. et al. Presence of luminal neutrophil extracellular traps in atherosclerosis. Thrombosis and Haemostasis 107, 597-598, doi:10.1160/TH11-09-0650 (2012).

177 Drechsler, M., Megens, R. T., van Zandvoort, M., Weber, C. \& Soehnlein, O. Hyperlipidemia-triggered neutrophilia promotes early atherosclerosis. Circulation 122, 1837-1845, doi:10.1161/CIRCULATIONAHA.110.961714 (2010).

178 van Leeuwen, M. et al. Accumulation of myeloperoxidase-positive neutrophils in atherosclerotic lesions in LDLR-/- mice. Arterioscler Thromb Vasc Biol 28, 84-89, doi:10.1161/ATVBAHA.107.154807 (2008).

179 Rotzius, P. et al. Distinct infiltration of neutrophils in lesion shoulders in ApoE/- mice. Am J Pathol 177, 493-500, doi:10.2353/ajpath.2010.090480 (2010).

180 Zernecke, A. et al. Protective role of CXC receptor 4/CXC ligand 12 unveils the importance of neutrophils in atherosclerosis. Circ Res 102, 209-217, doi:10.1161/CIRCRESAHA.107.160697 (2008).

181 Yvan-Charvet, L. et al. Increased inflammatory gene expression in ABC transporter-deficient macrophages: free cholesterol accumulation, increased signaling via toll-like receptors, and neutrophil infiltration of atherosclerotic lesions. Circulation 118, 1837-1847, doi:10.1161/CIRCULATIONAHA.108.793869 (2008).

182 Horckmans, M. et al. Neutrophils orchestrate post-myocardial infarction healing by polarizing macrophages towards a reparative phenotype. Eur Heart J 38, 187-197, doi:10.1093/eurheartj/ehw002 (2017).

183 Eghbalzadeh, K. et al. Compromised Anti-inflammatory Action of Neutrophil Extracellular Traps in PAD4-Deficient Mice Contributes to Aggravated Acute Inflammation After Myocardial Infarction. Front Immunol 10, 2313, doi:10.3389/fimmu.2019.02313 (2019). 
184 Summers, C. et al. Neutrophil kinetics in health and disease. Trends Immunol 31, 318-324, doi:10.1016/j.it.2010.05.006 (2010).

185 Jorch, S. K. \& Kubes, P. An emerging role for neutrophil extracellular traps in noninfectious disease. Nat Med 23, 279-287, doi:10.1038/nm.4294 (2017).

186 Soehnlein, O., Steffens, S., Hidalgo, A. \& Weber, C. Neutrophils as protagonists and targets in chronic inflammation. Nat Rev Immunol 17, 248261, doi:10.1038/nri.2017.10 (2017).

187 Brinkmann, V. et al. Neutrophil extracellular traps kill bacteria. Science 303, 1532-1535, doi:10.1126/science.1092385 (2004).

188 Fuchs, T. A. et al. Novel cell death program leads to neutrophil extracellular traps. J Cell Biol 176, 231-241, doi:10.1083/jcb.200606027 (2007).

189 Yipp, B. G. \& Kubes, P. NETosis: how vital is it? Blood 122, 2784-2794, doi:10.1182/blood-2013-04-457671 (2013).

190 Papayannopoulos, V., Metzler, K. D., Hakkim, A. \& Zychlinsky, A. Neutrophil elastase and myeloperoxidase regulate the formation of neutrophil extracellular traps. J Cell Biol 191, 677-691, doi:10.1083/jcb.201006052 (2010).

191 Pilsczek, F. H. et al. A novel mechanism of rapid nuclear neutrophil extracellular trap formation in response to Staphylococcus aureus. J Immunol 185, 7413-7425, doi:10.4049/jimmunol.1000675 (2010).

192 Yousefi, S., Mihalache, C., Kozlowski, E., Schmid, I. \& Simon, H. U. Viable neutrophils release mitochondrial DNA to form neutrophil extracellular traps. Cell Death Differ 16, 1438-1444, doi:10.1038/cdd.2009.96 (2009).

193 Yipp, B. G. et al. Infection-induced NETosis is a dynamic process involving neutrophil multitasking in vivo. Nat Med 18, 1386-1393, doi:10.1038/nm.2847 (2012).

194 Qi, H., Yang, S. \& Zhang, L. Neutrophil Extracellular Traps and Endothelial Dysfunction in Atherosclerosis and Thrombosis. Front Immunol 8, 928, doi:10.3389/fimmu.2017.00928 (2017).

195 Serhan, C. N. \& Savill, J. Resolution of inflammation: the beginning programs the end. Nat Immunol 6, 1191-1197, doi:10.1038/ni1276 (2005).

196 Van Avondt, K. \& Hartl, D. Mechanisms and disease relevance of neutrophil extracellular trap formation. Eur J Clin Invest 48 Suppl 2, e12919, doi:10.1111/eci.12919 (2018).

197 Neeli, I., Khan, S. N. \& Radic, M. Histone deimination as a response to inflammatory stimuli in neutrophils. J Immunol 180, 1895-1902, doi:10.4049/jimmunol.180.3.1895 (2008).

198 Wang, Y. et al. Histone hypercitrullination mediates chromatin decondensation and neutrophil extracellular trap formation. J Cell Biol 184, 205-213, doi:10.1083/jcb.200806072 (2009).

199 Leshner, M. et al. PAD4 mediated histone hypercitrullination induces heterochromatin decondensation and chromatin unfolding to form neutrophil extracellular trap-like structures. Front Immunol 3, 307, doi:10.3389/fimmu.2012.00307 (2012).

200 Metzler, K. D. et al. Myeloperoxidase is required for neutrophil extracellular trap formation: implications for innate immunity. Blood 117, 953-959, doi:10.1182/blood-2010-06-290171 (2011).

201 Metzler, K. D., Goosmann, C., Lubojemska, A., Zychlinsky, A. \& Papayannopoulos, V. A myeloperoxidase-containing complex regulates 
neutrophil elastase release and actin dynamics during NETosis. Cell Rep 8, 883-896, doi:10.1016/j.celrep.2014.06.044 (2014).

202 Chen, K. W. et al. Noncanonical inflammasome signaling elicits gasdermin Ddependent neutrophil extracellular traps. Sci Immunol 3, doi:10.1126/sciimmunol.aar6676 (2018).

203 Sollberger, G. et al. Gasdermin D plays a vital role in the generation of neutrophil extracellular traps. Sci Immunol 3, doi:10.1126/sciimmunol.aar6689 (2018).

204 Douda, D. N., Khan, M. A., Grasemann, H. \& Palaniyar, N. SK3 channel and mitochondrial ROS mediate NADPH oxidase-independent NETosis induced by calcium influx. Proc Natl Acad Sci U S A 112, 2817-2822, doi:10.1073/pnas.1414055112 (2015).

205 Lood, C. et al. Neutrophil extracellular traps enriched in oxidized mitochondrial DNA are interferogenic and contribute to lupus-like disease. Nat Med 22, 146153, doi:10.1038/nm.4027 (2016).

206 Boe, D. M., Curtis, B. J., Chen, M. M., Ippolito, J. A. \& Kovacs, E. J. Extracellular traps and macrophages: new roles for the versatile phagocyte. $J$ Leukoc Biol 97, 1023-1035, doi:10.1189/jlb.4RI1014-521R (2015).

207 Mollerherm, H., von Kockritz-Blickwede, M. \& Branitzki-Heinemann, K. Antimicrobial Activity of Mast Cells: Role and Relevance of Extracellular DNA Traps. Front Immunol 7, 265, doi:10.3389/fimmu.2016.00265 (2016).

208 Khandpur, R. et al. NETs are a source of citrullinated autoantigens and stimulate inflammatory responses in rheumatoid arthritis. Sci Transl Med 5, 178ra140, doi:10.1126/scitranslmed.3005580 (2013).

209 Cools-Lartigue, J., Spicer, J., Najmeh, S. \& Ferri, L. Neutrophil extracellular traps in cancer progression. Cell Mol Life Sci 71, 4179-4194, doi:10.1007/s00018-014-1683-3 (2014).

210 Wong, S. L. et al. Diabetes primes neutrophils to undergo NETosis, which impairs wound healing. Nat Med 21, 815-819, doi:10.1038/nm.3887 (2015).

211 Doring, Y., Soehnlein, O. \& Weber, C. Neutrophil Extracellular Traps in Atherosclerosis and Atherothrombosis. Circ Res 120, 736-743, doi:10.1161/CIRCRESAHA.116.309692 (2017).

212 Moschonas, I. C. \& Tselepis, A. D. The pathway of neutrophil extracellular traps towards atherosclerosis and thrombosis. Atherosclerosis 288, 9-16, doi:10.1016/j.atherosclerosis.2019.06.919 (2019).

213 Range, $\mathrm{H}$. et al. Periodontal bacteria in human carotid atherothrombosis as a potential trigger for neutrophil activation. Atherosclerosis 236, 448-455, doi:10.1016/j.atherosclerosis.2014.07.034 (2014).

214 Carestia, A. et al. Mediators and molecular pathways involved in the regulation of neutrophil extracellular trap formation mediated by activated platelets. $J$ Leukoc Bio/ 99, 153-162, doi:10.1189/jlb.3A0415-161R (2016).

215 Awasthi, D. et al. Oxidized LDL induced extracellular trap formation in human neutrophils via TLR-PKC-IRAK-MAPK and NADPH-oxidase activation. Free Radic Biol Med 93, 190-203, doi:10.1016/j.freeradbiomed.2016.01.004 (2016).

216 Warnatsch, A., loannou, M., Wang, Q. \& Papayannopoulos, V. Inflammation. Neutrophil extracellular traps license macrophages for cytokine production in atherosclerosis. Science 349, 316-320, doi:10.1126/science.aaa8064 (2015).

217 Borissoff, J. I. et al. Elevated levels of circulating DNA and chromatin are independently associated with severe coronary atherosclerosis and a 
prothrombotic state. Arterioscler Thromb Vasc Biol 33, 2032-2040, doi:10.1161/ATVBAHA.113.301627 (2013).

218 Oklu, R., Stone, J. R., Albadawi, H. \& Watkins, M. T. Extracellular traps in lipid-rich lesions of carotid atherosclerotic plaques: implications for lipoprotein retention and lesion progression. J Vasc Interv Radiol 25, 631-634, doi:10.1016/j.jvir.2013.12.567 (2014).

219 Quillard, T. et al. TLR2 and neutrophils potentiate endothelial stress, apoptosis and detachment: implications for superficial erosion. Eur Heart J 36, 13941404, doi:10.1093/eurheartj/ehv044 (2015).

220 Naruko, T. et al. Neutrophil infiltration of culprit lesions in acute coronary syndromes. Circulation 106, 2894-2900, doi:10.1161/01.cir.0000042674.89762.20 (2002).

221 Pertiwi, K. R. et al. Neutrophil Extracellular Traps Participate in All Different Types of Thrombotic and Haemorrhagic Complications of Coronary Atherosclerosis. Thromb Haemost 118, 1078-1087, doi:10.1055/s-00381641749 (2018).

222 Westerterp, M. et al. Cholesterol Efflux Pathways Suppress Inflammasome Activation, NETosis, and Atherogenesis. Circulation 138, 898-912, doi:10.1161/CIRCULATIONAHA.117.032636 (2018).

223 Liu, Y. et al. Myeloid-Specific Deletion of Peptidylarginine Deiminase 4 Mitigates Atherosclerosis. Front Immunol 9, 1680, doi:10.3389/fimmu.2018.01680 (2018).

224 Knight, J. S. et al. Peptidylarginine deiminase inhibition reduces vascular damage and modulates innate immune responses in murine models of atherosclerosis. Circ Res 114, 947-956, doi:10.1161/CIRCRESAHA.114.303312 (2014).

225 Hansson, G. K. \& Hermansson, A. The immune system in atherosclerosis. Nat Immunol 12, 204-212, doi:10.1038/ni.2001 (2011).

226 Podrez, E. A., Schmitt, D., Hoff, H. F. \& Hazen, S. L. Myeloperoxidasegenerated reactive nitrogen species convert LDL into an atherogenic form in vitro. J Clin Invest 103, 1547-1560, doi:10.1172/JCI5549 (1999).

227 Park, Y. M., Febbraio, M. \& Silverstein, R. L. CD36 modulates migration of mouse and human macrophages in response to oxidized LDL and may contribute to macrophage trapping in the arterial intima. J Clin Invest 119, 136145, doi:10.1172/JCl35535 (2009).

228 Smith, C. K. et al. Neutrophil extracellular trap-derived enzymes oxidize highdensity lipoprotein: an additional proatherogenic mechanism in systemic lupus erythematosus. Arthritis Rheumatol 66, 2532-2544, doi:10.1002/art.38703 (2014).

229 Kougias, P. et al. Defensins and cathelicidins: neutrophil peptides with roles in inflammation, hyperlipidemia and atherosclerosis. J Cell Mol Med 9, 3-10, doi:10.1111/j.1582-4934.2005.tb00332.x (2005).

230 Wang, J. et al. Cathepsin G activity lowers plasma $\mathrm{LDL}$ and reduces atherosclerosis. Biochim Biophys Acta 1842, 2174-2183, doi:10.1016/j.bbadis.2014.07.026 (2014).

231 Soehnlein, O. et al. Neutrophil secretion products pave the way for inflammatory monocytes. Blood 112, 1461-1471, doi:10.1182/blood-2008-02139634 (2008). 
232 Wantha, S. et al. Neutrophil-derived cathelicidin promotes adhesion of classical monocytes. Circ Res 112, 792-801, doi:10.1161/CIRCRESAHA.112.300666 (2013).

233 Lande, R. et al. Neutrophils activate plasmacytoid dendritic cells by releasing self-DNA-peptide complexes in systemic lupus erythematosus. Sci Transl Med 3, 73ra19, doi:10.1126/scitranslmed.3001180 (2011).

234 Doring, Y. et al. Auto-antigenic protein-DNA complexes stimulate plasmacytoid dendritic cells to promote atherosclerosis. Circulation 125, 16731683, doi:10.1161/CIRCULATIONAHA.111.046755 (2012).

235 Menegazzo, L. et al. NETosis is induced by high glucose and associated with type 2 diabetes. Acta Diabetol 52, 497-503, doi:10.1007/s00592-014-0676-x (2015).

236 Rodriguez-Espinosa, O., Rojas-Espinosa, O., Moreno-Altamirano, M. M., Lopez-Villegas, E. O. \& Sanchez-Garcia, F. J. Metabolic requirements for neutrophil extracellular traps formation. Immunology 145, 213-224, doi:10.1111/imm.12437 (2015).

237 Parathath, S. et al. Diabetes adversely affects macrophages during atherosclerotic plaque regression in mice. Diabetes 60, 1759-1769, doi:10.2337/db10-0778 (2011).

238 Fadini, G. P. et al. NETosis Delays Diabetic Wound Healing in Mice and Humans. Diabetes 65, 1061-1071, doi:10.2337/db15-0863 (2016).

239 Franck, G. et al. Roles of PAD4 and NETosis in Experimental Atherosclerosis and Arterial Injury: Implications for Superficial Erosion. Circ Res 123, 33-42, doi:10.1161/CIRCRESAHA.117.312494 (2018).

240 Jang, B. et al. Peptidylarginine deiminase inhibition impairs Toll-like receptor agonist-induced functional maturation of dendritic cells, resulting in the loss of T cell-proliferative capacity: a partial mechanism with therapeutic potential in inflammatory settings. J Leukoc Biol 97, 351-362, doi:10.1189/jlb.3A0314142RR (2015).

241 Park, B., Yim, J. H., Lee, H. K., Kim, B. O. \& Pyo, S. Ramalin inhibits VCAM-1 expression and adhesion of monocyte to vascular smooth muscle cells through MAPK and PADI4-dependent NF-kB and AP-1 pathways. Biosci Biotechnol Biochem 79, 539-552, doi:10.1080/09168451.2014.991681 (2015).

242 Lewis, H. D. et al. Inhibition of PAD4 activity is sufficient to disrupt mouse and human NET formation. Nat Chem Biol 11, 189-191, doi:10.1038/nchembio.1735 (2015).

$243 \mathrm{Li}, \mathrm{P}$. et al. PAD4 is essential for antibacterial innate immunity mediated by neutrophil extracellular traps. J Exp Med 207, 1853-1862, doi:10.1084/jem.20100239 (2010).

244 Martinod, K. et al. PAD4-deficiency does not affect bacteremia in polymicrobial sepsis and ameliorates endotoxemic shock. Blood 125, 1948-1956, doi:10.1182/blood-2014-07-587709 (2015).

245 Pham, C. T., Ivanovich, J. L., Raptis, S. Z., Zehnbauer, B. \& Ley, T. J. Papillon-Lefevre syndrome: correlating the molecular, cellular, and clinical consequences of cathepsin C/dipeptidyl peptidase I deficiency in humans. $J$ Immunol 173, 7277-7281, doi:10.4049/jimmunol.173.12.7277 (2004).

246 Sorensen, O. E. et al. Papillon-Lefevre syndrome patient reveals speciesdependent requirements for neutrophil defenses. J Clin Invest 124, 45394548, doi:10.1172/JCl76009 (2014). 
247 Vitkov, L., Hartl, D., Minnich, B. \& Hannig, M. Janus-Faced Neutrophil Extracellular Traps in Periodontitis. Front Immunol 8, 1404, doi:10.3389/fimmu.2017.01404 (2017).

248 Mogayzel, P. J., Jr. et al. Cystic fibrosis pulmonary guidelines. Chronic medications for maintenance of lung health. Am J Respir Crit Care Med 187, 680-689, doi:10.1164/rccm.201207-1160oe (2013).

249 Hakkim, A. et al. Impairment of neutrophil extracellular trap degradation is associated with lupus nephritis. Proc Natl Acad Sci U S A 107, 9813-9818, doi:10.1073/pnas.0909927107 (2010).

250 Yamamoto, K. et al. Augmented neutrophil extracellular traps formation promotes atherosclerosis development in socially defeated apoE(-/-) mice.

Biochem Biophys Res Commun 500, 490-496, doi:10.1016/j.bbrc.2018.04.115 (2018).

251 Yasutomo, K. et al. Mutation of DNASE1 in people with systemic lupus erythematosus. Nat Genet 28, 313-314, doi:10.1038/91070 (2001).

252 Bodano, A., Amarelo, J., Gonzalez, A., Gomez-Reino, J. J. \& Conde, C. Novel DNASE I mutations related to systemic lupus erythematosus. Arthritis Rheum 50, 4070-4071, doi:10.1002/art.20721 (2004).

253 Zhu, B. et al. Increased DNase I activity in diabetes might be associated with injury of pancreas. Mol Cell Biochem 393, 23-32, doi:10.1007/s11010-0142043-1 (2014).

254 Jimenez-Alcazar, M. et al. Host DNases prevent vascular occlusion by neutrophil extracellular traps. Science 358, 1202-1206, doi:10.1126/science.aam8897 (2017).

255 Kolaczkowska, E. et al. Molecular mechanisms of NET formation and degradation revealed by intravital imaging in the liver vasculature. Nat Commun 6, 6673, doi:10.1038/ncomms7673 (2015).

256 Soehnlein, O., Ortega-Gomez, A., Doring, Y. \& Weber, C. Neutrophilmacrophage interplay in atherosclerosis: protease-mediated cytokine processing versus NET release. Thromb Haemost 114, 866-867, doi:10.1160/TH15-08-0623 (2015). 


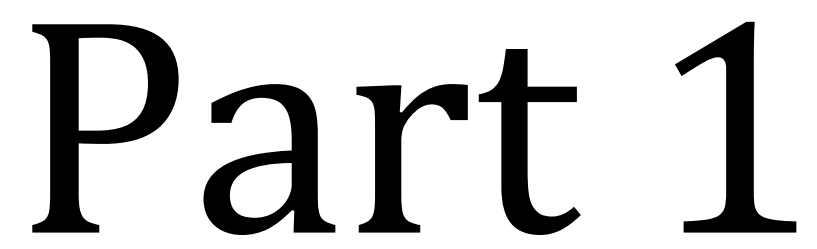

Diabetic Dyslipidemia

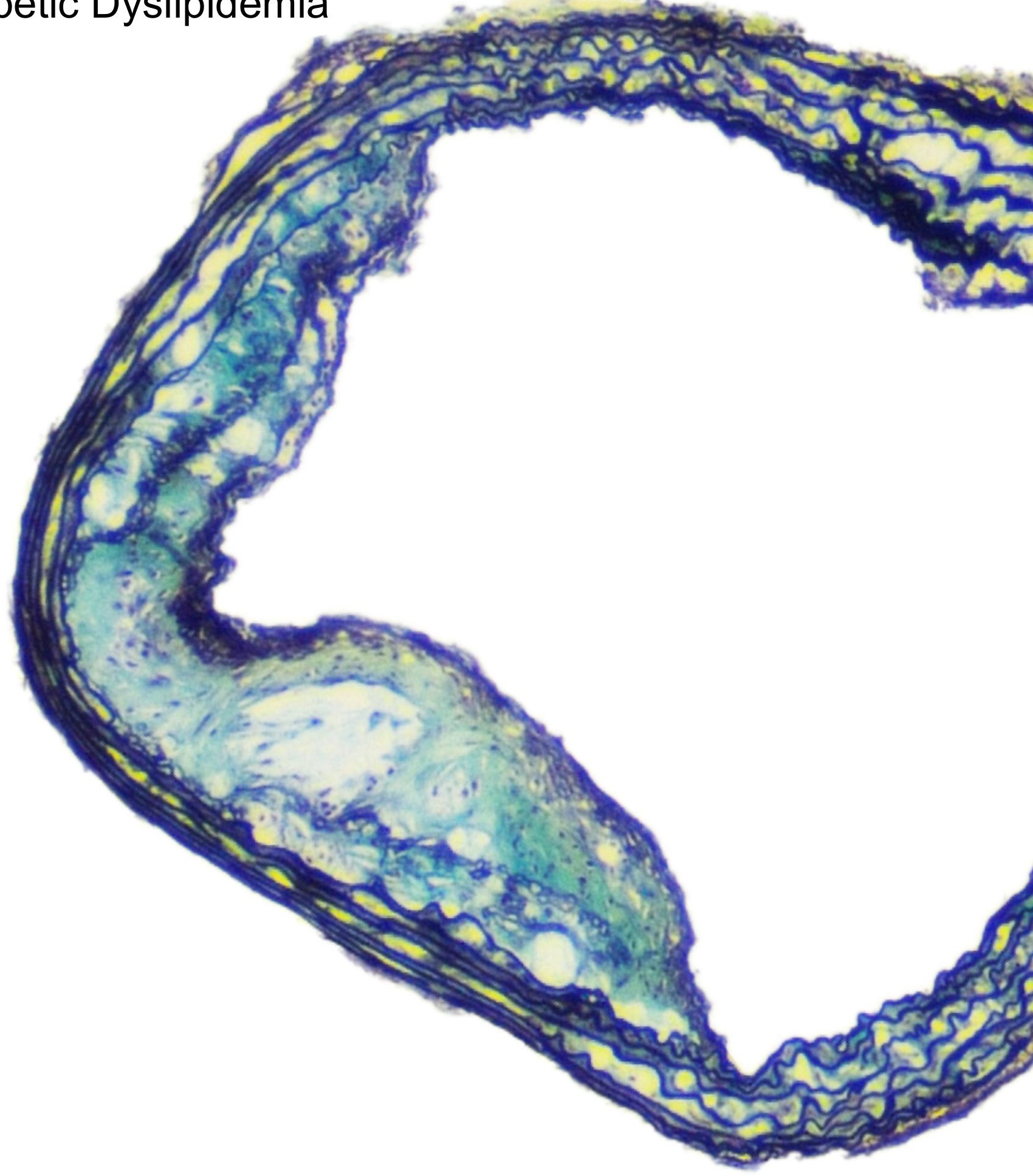




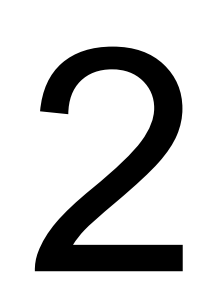

\section{High-density Lipoprotein Cholesterol Efflux Capacity is not associated with Atherosclerosis and Prevalence of Cardiovascular Outcome: The CODAM Study}

Tatjana Josefs, Kristiaan Wouters, Uwe J.F. Tietge, Wijtske Annema, Robin P.F. Dullaart, Tomas Vaisar, llja C.W. Arts, Carla J.H. van der Kallen, Coen D.A. Stehouwer, Casper G. Schalkwijk, Ira J. Goldberg, Edward A. Fisher, Marleen M.J. van Greevenbroek 


\begin{abstract}
Background: Cholesterol Efflux Capacity (CEC) is considered to be a key atheroprotective property of high-density lipoproteins (HDL). However, the role of HDL-CEC in atherosclerosis and cardiovascular (CV) risk is still controversial and data in individuals with diabetes are limited.
\end{abstract}

Objective: In this study, we have investigated the relationship of CEC and other HDL characteristics with clinical and subclinical atherosclerosis in subjects with elevated CVD risk and Type 2 Diabetes Mellitus (T2DM).

Methods: Using multiple linear regression analyses, we determined the relationship of HDL-CEC with carotid intima media thickness (cIMT, Z-Score), an endothelial dysfunction (EnD) Score (Z-Score), prevalent CV disease (CVD, n=150 cases) and history of CV events (CVE, $n=85$ cases) in an observational cohort (CODAM, $n=574$, $59.6 \pm 0.3 \mathrm{yr}, 61.3 \% \mathrm{men}, 24.4 \% \mathrm{~T} 2 \mathrm{DM})$. Stratified analyses were performed to determine if the associations differed between individuals with normal glucose metabolism (NGM) and those with a disturbed glucose metabolism.

Results: HDL-CEC was not associated with either marker of atherosclerosis (cIMT, EnD Score) nor with CVD or CVE. In contrast, other HDL characteristics i.e. HDL-Cholesterol (HDL-C, Z-Score), apolipoprotein A-I (apoA-I, Z-Score), HDL size (Z-Score) and HDL particle number (HDL-P, Z-Score) were inversely and significantly associated with the EnD Score (ßs -0.226 to $-0.097, p<0.05$ ) and CVE (ORs 0.61 to 0.68, $p<0.05)$. In stratified analyses, HDL size and HDL-P were significantly associated with the EnD Score in individuals with NGM (pinteraction 0.039 and 0.005 , respectively), but not in those with (pre)diabetes. HDL-C and apoA-I were inversely associated with prevalent CVD in individuals with (pre)diabetes ( $p_{\text {interaction }}=0.074$ and 0.034 , respectively), but not in those with NGM.

Conclusion: HDL-CEC is not associated with clinicial or subclinical atherosclerosis, neither in the whole population nor in indviduals with (pre)diabetes, while other HDL characteristics show atheroprotective associations. The atheroprotective associations of HDL-size and HDL-P are lost in (pre)diabetes, while higher concentrations of HDL-C and apoA-I are associated with lower prevalence of CVD in (pre)diabetes. 


\section{Introduction}

High-density Lipoprotein (HDL) is well-known for its cardioprotective effect, resulting from, among others, its anti-inflammatory and endothelial protective properties. The key cardioprotective effect of HDL is thought to originate in its cholesterol efflux capacity (HDL-CEC), which represents the first step in reverse cholesterol transport ${ }^{1,2}$. Since up till now, the results of HDL Cholesterol (HDL-C) raising trials have been disappointing ${ }^{3,4}$, the importance of HDL function in cardiovascular diseases (CVD) has become a topic of major interest over the last few years.

HDL-mediated cholesterol efflux may lead to cholesterol reduction in the atherosclerotic plaque by promoting the removal of excess cholesterol from plaque macrophage foam cells and transporting it back to the liver for its excretion into the bile. $^{5,6}$ So far, animal studies showed a link between macrophage-specific cholesterol efflux and prevention of atherosclerosis. ${ }^{7-10}$ Further, functional HDL was shown to promote atherosclerosis regression. ${ }^{11,12}$ However, it has also been shown that, besides anti-inflammatory effects ${ }^{13}$, cholesterol efflux can also exert pro-inflammatory effects on mouse and human macrophages, which are thought to be detrimental for atherosclerosis. ${ }^{14,15}$ In human studies an inverse association between HDL-CEC and the prevalence ${ }^{16}$ and incidence of $C V$ events $(C V E)^{17-19}$ was shown, which remained present after adjustment for CV risk factors, including HDL-C ${ }^{19}, \mathrm{HDL}-\mathrm{C}$ and apoA-I ${ }^{16,18}$, and HDL-C and HDL particle number (HDL-P) ${ }^{17}$. In contrast, another study showed that a higher HDL-CEC was associated with increased risk of incident CVE, also after adjustment for CV risk factors, including HDL-C and LDL-C. ${ }^{20}$ The reasons for these apparent discrepancies are currently unclear.

Diabetes increases the risk of CVD by 2 to 4 -fold. ${ }^{21}$ While the underlying mechanisms remain to be fully understood, altered levels or impaired functions of circulating lipoproteins are likely to be involved. Some studies have shown that HDL-CEC is impaired in patients with insulin resistance ${ }^{22}$ and type 2 diabetes mellitus $(\text { T2DM })^{18,23}$, but other studies did not find any difference ${ }^{24}$ or even increased HDL-CEC compared to healthy controls. ${ }^{25-29}$ While CV outcome studies demonstrated that HDL-CEC did not differ between non-T2DM and T2DM subjects, they showed that statin treatment in both groups leads to an increase in HDL-CEC, which correlates with beneficial changes on plaque morphology. ${ }^{30,31}$ 
Taken together, the outcomes of human studies on the relationship between HDL-CEC and CVD have been controversial and data in individuals with diabetes are limited. The goal of this study was to evaluate the associations of HDL-CEC and other characteristics of $\mathrm{HDL}$ on (sub)clinical atherosclerotic disease, as represented by carotid intima media thickness (cIMT) and an endothelial dysfunction Score (EnD Score), prevalent CVD and history of CVE. In addition, we determined whether such associations differ between individuals with normal glucose metabolism (NGM) and those with prediabetes or diabetes (hereafter referred to as (pre)diabetes) and are the first to report on the relationship of CEC with CVD/CVE in individuals with (pre)diabetes. This evaluation was done in a well-established human cohort that is characterized by a moderately increased risk of cardiometabolic disease, i.e., the Cohort on Diabetes and Atherosclerosis Maastricht (CODAM). We hypothesized that a higher HDL-CEC is associated with less (sub)clinical atherosclerotic disease and that this association is attenuated in individuals with (pre)diabetes.

\section{Patients \& Methods}

\section{Study Population}

CODAM includes 574 participants who were selected from a large cohort in the general population $(>20,000)$ based on an elevated risk of T2DM and CVD as described elsewhere. ${ }^{32}$ Inclusion criteria were Caucasian descent, age $>40$ years, and one or more cardiometabolic risk factors (i.e. BMI $>25 \mathrm{~kg} / \mathrm{m}^{2}$, use of anti-hypertensive medication, positive family history of T2DM, history of gestational diabetes and/or glycosuria). The CODAM study was approved by the Medical Ethics Committee of the Maastricht University Medical Centre. All participants gave written informed consent and the described methods were carried out in accordance with the approved guidelines. Of the 574 participants, 25 had missing data on HDL characteristics and 16 had missing covariates leaving $n=533$ for a complete case analyses with the $E n D$ Score, CVD or CVE as outcomes (NGM n=279, (pre)diabetes $n=254$ ). $N=37$ also had missing data on cIMT leaving $n=496$ (NGM n=268, (pre)diabetes =228).

All participants were asked to stop their lipid-modifying medication 14 days and all other medications on the day before the measurements ( $>80 \%$ adherence). Blood samples were obtained by venipuncture after an overnight fast and plasma aliquots were stored at $-80^{\circ} \mathrm{C}$ until use. All participants underwent an oral glucose tolerance 
test (except those with established T2DM), and were categorized as having normal glucose metabolism (NGM), impaired glucose metabolism (IGM, i.e. prediabetes) or T2DM as previously described. ${ }^{33,34,32}$ Glycated hemoglobin $\left(\mathrm{HbA}_{1 \mathrm{c}}\right)$ was determined as previously described. ${ }^{25}$

\section{Cardiovascular Measures}

Carotid Intima-Media Thickness (cIMT)

cIMT was measured at the left and right common carotid artery $10-20 \mathrm{~mm}$ proximal to the carotid bulb with an ultrasound imaging device as reported previously. ${ }^{35}$

\section{Endothelial Dysfunction (EnD) Score}

Soluble intercellular adhesion molecule (sICAM-1), soluble vascular cell adhesion molecule (sVCAM-1), and soluble endothelial selectin (sESel) were measured using ELISA assays in serum or EDTA plasma. The measurements were calibrated after cross-validation as described elsewhere. ${ }^{36}$ Baseline von Willebrand factor (VWF) was measured with an in-house ELISA in citrated plasma as described. ${ }^{37}$

\section{Cardiovascular Events (CVE)}

CVE were defined as the occurrence of myocardial infarction (MI), coronary bypass, percutaneous coronary intervention and /or stroke reported by questionnaires; as well as signs of $\mathrm{MI}$ on an electrocardiogram (Minnesota codes 1-1 or 1-2), as reported earlier. ${ }^{32}$

\section{Cardiovascular Disease (CVD)}

Prior CVD was defined by self-reported history of CVE. CVD additionally included signs of coronary ischemia (Minnesota codes 1-3, 4-1, 4-2, 4-3, 5-1, 5-2, 5-3, or 7-1); non-traumatic limb amputation; and/or ankle-brachial index $<0.9 .{ }^{32}$

\section{HDL Characteristics}

HDL-C was determined in EDTA plasma using the HDL-C plus assay (Roche Diagnostics, Mannheim, Germany) and apoA-I was determined by immunoephelometric assays as reported earlier. ${ }^{32}$ HDL size and HDL-P were 
measured using nuclear magnetic resonance spectroscopy as described previously (available at: www.nightingalehealth.com). ${ }^{38}$

\section{Cholesterol Efflux Capacity (HDL-CEC)}

Measurement of Cholesterol Efflux Capacity was done exactly as described elsewhere. ${ }^{24}$ Briefly, HDL-CEC towards apoB-depleted plasma was measured using THP-1 human monocytes that were differentiated into macrophages by the addition of $100 \mathrm{nM}$ phorbol myristate acetate (PMA). ${ }^{39,40}$ Differentiated THP-1 macrophages were then loaded with acetylated LDL $(50 \mu \mathrm{g}$ protein/ $\mathrm{mL})$ and $1 \mu \mathrm{Ci} / \mathrm{ml}^{3} \mathrm{H}$-cholesterol (Perkin Elmer, Boston, MA) for 24 hours followed by equilibration for 24 hours in RPMI 1640 medium containing $2 \%$ bovine serum albumin. ${ }^{40}$ After equilibration, efflux towards $2 \%$ apoB-depleted plasma was performed for 5 hours. ApoB-depleted plasma was generated by precipitating apoB-containing lipoproteins using polyethylene glycol (PEG 600, Sigma, St Louis, MO) in 10mM HEPES (pH $08.0=$ according to a commonly used protocol. ${ }^{16-18,24,41}$ CEC measurements were performed in duplicates and the same time using the same reagents to limit potential variations due to different assay conditions. Values for HDL-CEC were normalized using a standard curve of different concentrations of pooled apoB-depleted control plasma to correct for potential inter-assay variation across plates, and expressed in arbitrary units. ${ }^{41}$ The intra-assay CV of the method was previously determined to be $5.4 \%$, the inter-assay CV $7.9 \%$. 24,41

\section{Other covariates}

BMI was calculated as weight $(\mathrm{kg}) /$ height $(\mathrm{m})$ squared. ${ }^{42}$ Waist circumference was measured as before.$^{35}$ Smoking behavior, physical activity ${ }^{43}$, dietary calorie intake ${ }^{33}$, use of anti-hypertensive, glucose-lowering, and lipid-medication ${ }^{42}$ was assessed with on-site administered questionnaires. Blood pressure was measured as described before. ${ }^{24}$ LDL-C was calculated with the Friedewald formula. ${ }^{32}$ Triglycerides (TG) and apoB were determined as reported earlier. ${ }^{32}$

\section{Statistical analyses}

Categorical variables are presented as frequencies and percentages. Normally distributed continuous variables are presented as mean \pm standard deviation, skewed 
variables are presented as median with interquartile range (IQR). Skewed variables (i.e. fasting plasma glucose (FPG), HDL-P, TG and EnD markers) were In-transformed to achieve normal distribution prior to analysis.

To define an overall measure of endothelial dysfunction, a composite score was calculated using sICAM-1, SVCAM-1, sESel and VWF. For this, each individual In-transformed biomarker measurements were first standardized and the individual $Z$-Scores were averaged into the endothelial dysfunction score (EnD Score). To allow direct comparison of the effect sizes for the EnD Score and cIMT, CIMT was also standardized. For the same reason, $\mathrm{HDL}$ characteristics were also converted into their respective Z-Scores.

Cross-sectional associations of HDL characteristics (HDL-CEC, HDL-C, apoA-I, HDL size, HDL-P) with the EnD Score $(\mathrm{N}=533)$ and with cIMT $(\mathrm{N}=496)$ were investigated using linear regression analysis. Logistic regression analyses were done for CVD ( $N=533,150$ CVD cases) and CVE ( $N=533,85$ CVE cases). All regression coefficients are presented as crude effect sizes (Model 1 ) and adjusted for several potential confounders: Model 2: adjusted for age (yrs) and sex, Model 3 (full model): additionally adjusted for smoking (\%), physical activity (mets/day), caloric intake ( $\mathrm{KJ} /$ day), use of glucose-lowering, lipid-modifying, and antihypertensive medication (all yes $/ \mathrm{no})$, BMI $\left(\mathrm{kg} / \mathrm{m}^{2}\right)$, systolic blood pressure $(\mathrm{mmHg})$, diastolic blood pressure ( $\mathrm{mmHg}$ ), fasting plasma glucose (mmol/L), IGM (yes/no), T2DM (yes/no).

To determine if the associations differed between individuals with NGM and those with a disturbed glucose metabolism (pre-diabetes or diabetes), we repeated the analyses mentioned above stratified according to normal or disturbed glucose metabolism. We also performed an interaction analysis by adding interaction terms (HDL characteristic (e.g. HDL-CEC) $x$ glucose metabolism (normal or disturbed)) to the main analyses.

HDL-CEC was significantly higher in women compared to men, but the strength of association of HDL-CEC with cIMT, EnD Score, CVD and CVE did not differ between women and men.

Statistical analyses were performed using IBM SPSS Statistics Version 23. For the main effects $P<0.05$, and for interaction terms $P_{\text {interaction }}<0.10$ was considered statistically significant. 
Chapter 2

\section{Results}

The CODAM study population

The overall characteristics of the CODAM study as a whole and across tertiles of HDL-CEC are shown in Table 1. For the total population, the mean age was $59.6 \pm 7.0$ yrs, $61.3 \%$ were men, and $24.2 \%$ had T2DM. Mean values for systolic blood pressure were $140.1 \pm 19.0 \mathrm{mmHg}$, diastolic blood pressure $81.6 \pm 9.1 \mathrm{mmHg}$ and $20 \%$ were current smokers. $28.1 \%$ and $16.0 \%$ were previously diagnosed with CVD and CVE, respectively. Prevalence of normal glucose metabolism, prediabetes or diabetes and $\mathrm{Hb} 1 \mathrm{Ac}$ levels were not significantly different among HDL-CEC tertiles while HDL-C, apoA-I, HDL size, and HDL-P were higher in those individuals who were in the highest CEC tertiles. cIMT, EnD, CVD and CVE did not differ among CEC tertiles.

The associations of HDL-CEC with the atherosclerosis surrogates (i.e. cIMT and EnD Score) are shown in Table 2. In crude analyses, clMT was not associated with HDL -CEC ( $\beta=-0.04,[95 \% \mathrm{Cl}-0.13 ; 0.05], p=0.413)$, but was significantly and inversely associated with HDL-C, apoA-I, and HDL-size, but not with HDL-P. These associations were attenuated and no longer significant in fully adjusted analyses (HDL-C: $\beta=-0.07$ [ -0.16; 0.02], $p=0.130 ;$ apoA-I: $\beta=-0.04$ [ $-0.13 ; 0.05] p=0.408 ;$ HDL size: $\beta=-0.03$ $[-0.12 ; 0.06] p=0.527)$. Likewise, HDL-CEC was not associated with the EnD Score, neither crude $(\beta=-0.01[-0.09 ; 0.08] p=0.897)$ nor after adjustment for potential confounders. In contrast, HDL-C, apoA-I, HDL size, and HDL-P, were all significantly and inversely associated with the EnD Score and remained so in the fully adjusted analyses (HDL-C: $\beta=-0.23$ [-0.31; -0.14] $p<0.001$, apoA-I: $\beta=-0.16 \quad[-0.24 ;-0.07]$ $p<0.001$, HDL size: $\beta=-0.12$ [-0.21; -0.03$], p=0.008$, HDL-P: $\beta=-0.10$ [-1.8; -0.01$]$, $\mathrm{p}=0.028)$.

Table 1: Values are mean \pm SD for continuous variables with normal distribution and median [Interquartile range] for continuous variables with skewed distribution.

* variables with skewed distribution were In-transformed prior to t-test, ${ }^{\dagger} n=496$, cIMT indicates carotid intima media thickness; EnD score, Endothelial Dysfunction Score; CVD, Cardiovascular Disease; CVE, CV Events; NGM, normal glucose metabolism; IGM, impaired GM; T2DM, Type 2 Diabetes Mellitus; $\mathrm{Hb} 1 \mathrm{Ac}$, hemoglobin A1c; FPG, fasting plasma glucose; BMI, body mass index; BP, blood pressure; CEC, cholesterol efflux capacity; HDL-C, high density lipoprotein Cholesterol; ApoA-I, apolipoprotein A-I; HDL-P, HDL particle number; LDL-C, low density lipoprotein Cholesterol; ApoB, apolipoprotein B; VLDL, very low density lipoprotein; TG, triglyceride. 
"ల

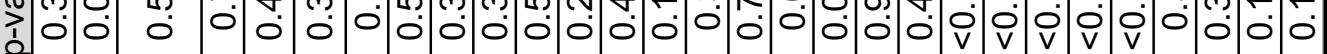

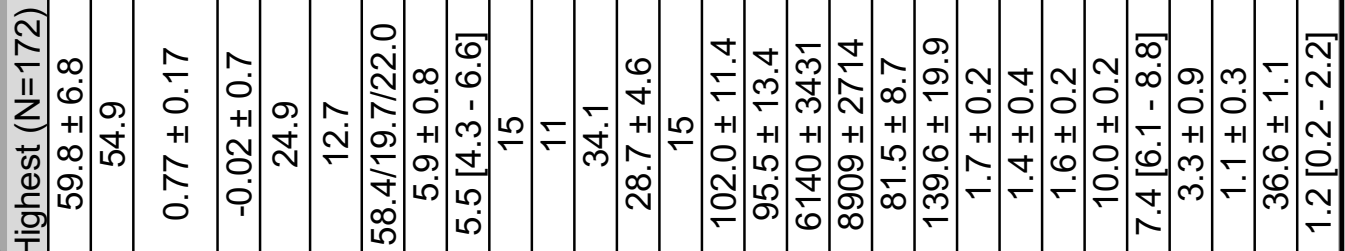

g主

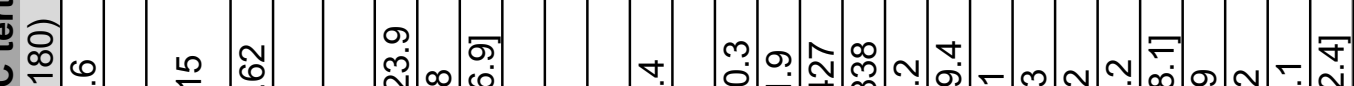

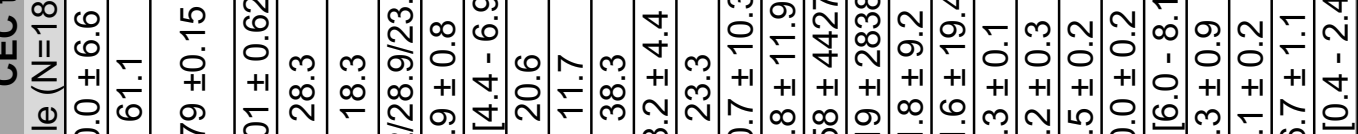

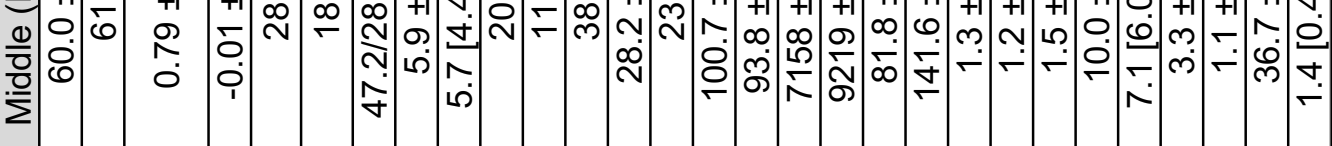

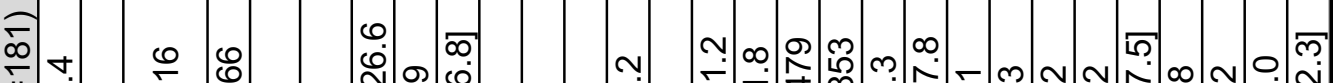

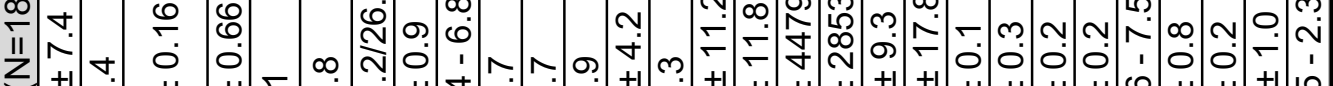

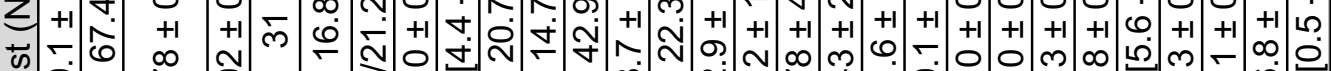

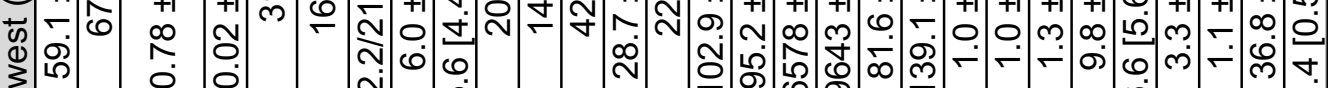

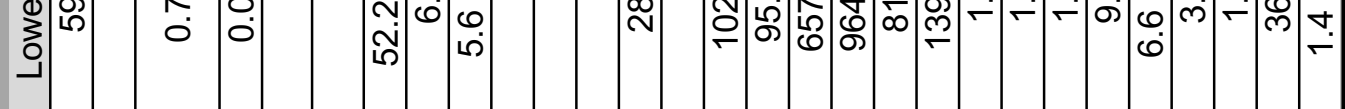

음

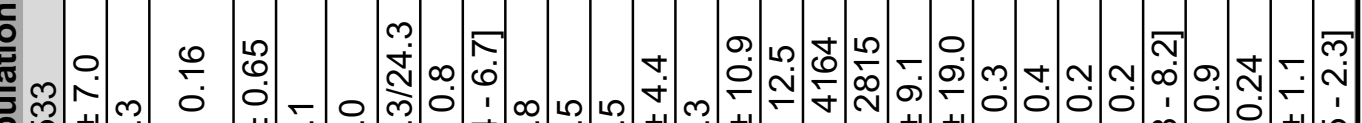

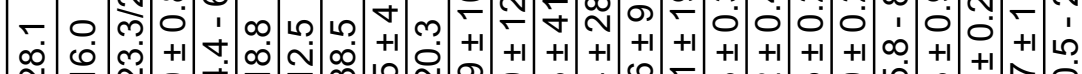

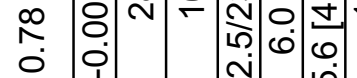

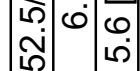

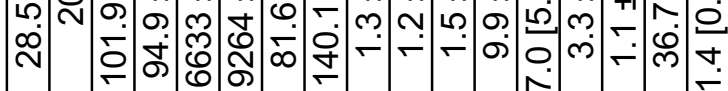

ก

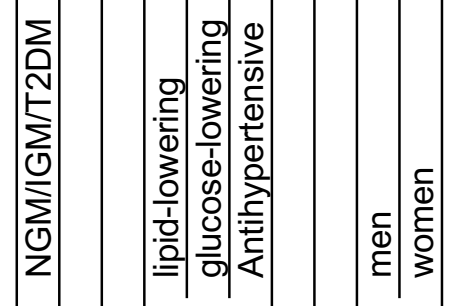


Because all the HDL characteristics differed between CEC tertiles (Table 1), we adjusted the analyses of CEC with cIMT and the EnD Score for HDL-C, apoA-I, HDL size and HDL-P (Suppl. Table S1). This did not change the lack of association of HDLCEC with cIMT or the EnD Score. We also evaluated if the associations of the individual HDL-characteristics with the atherosclerosis surrogates were independent of plasma triglyceride (TG) and LDL-C levels (Suppl. Table S2). When the analyses presented in Table 2 were additionally adjusted for TG or LDL-C, the associations between the HDL characteristics and cIMT remained essentially unaltered (Suppl. Table S2). Further, none of the previously observed inverse associations of HDL-C, apoA-I, HDL size and HDL-P with the EnD Score were affected by additional adjustment for LDL-C. Adjustment for TG did, however, slightly attenuate the associations of HDL-size and HDL-P with the EnD Score (for HDL size to $\beta=-0.08$ [$0.18 ; 0.02] p=0.103$, and for HDL-P to $\beta=-0.08[-0.17 ; 0.01] p=0.068)$.

Table 2. Relationship of HDL-CEC and other HDL characteristics with cIMT and the EnD Score.

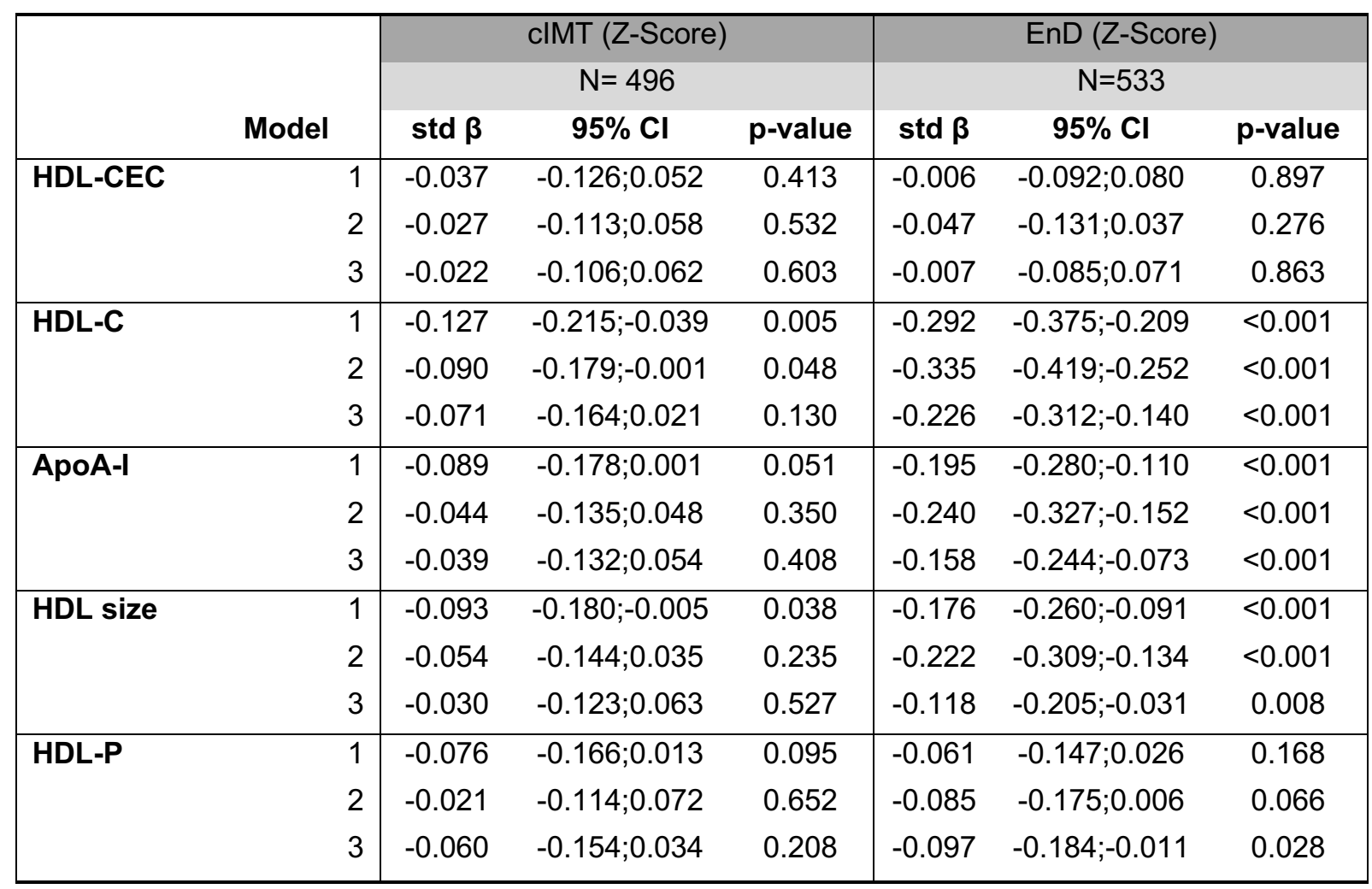


Table 2: Linear regression analyses, Model 1: crude, Model 2: adjusted for age, sex, Model 3 additionally adjusted for smoking, bmi, medication usage (antihypertensive, glucose- and lipid lowering), physical activity, caloric intake, systolic and diastolic blood pressure, fasting plasma glucose, and glucose metabolism state. Std $\beta$; standardized regression coefficient.

\section{Association of HDL characteristics with prevalence of CVD and CVE}

Next, we performed logistic regression analyses with prevalent CVD and CVE as outcomes. HDL-CEC was not associated with CVD or CVE (Figure 1). Also, none of the other HDL characteristics were associated with CVD (Figure 1, panel a). On the other hand, HDL-C (OR 0.61 [95\% Cl 0.41; 0.90] p=0.014), apoA-I (OR $0.67[95 \% \mathrm{Cl}$ $0.46 ; 0.96] p=0.030)$ and $\mathrm{HDL}-\mathrm{P}(\mathrm{OR} 0.87[95 \% \mathrm{Cl} 0.48 ; 0.97] \mathrm{p}=0.031)$ were inversely associated with prevalent CVE, whereas HDL size was not (Figure 1, panel b). This implies that one standard deviation higher HDL-C, apoA-I, and HDL-P were associated with approximately 1.6-, 1.5- and 1.2-fold fewer CVE cases, respectively. None of these associations were dependent on plasma TG levels or LDL-C (Suppl. Table S3).

Figure 1: Forrest plot indicating the relationship of HDL characteristics and prevalent cardiovascular disease (CVD) and CV events (CVE) risk in whole population as well as stratified analyses.

Logistic regression analyses were adjusted for age, sex, smoking, bmi, medication usage (antihypertensive, glucose- and lipid lowering), physical activity, caloric intake, systolic and diastolic blood pressure, fasting plasma glucose, and glucose metabolism state. CVD N=383, 150 CVD cases (NGM: $N=279,67$ CVD cases; (pre)diabetes: $N=253,83$ CVD cases) and CVE N=448, 85 CVE cases (NGM: $N=279,39$ CVE cases, (pre)diabetes: $N=254,46$ CVE cases). 
$\underline{1 a}$

CVD

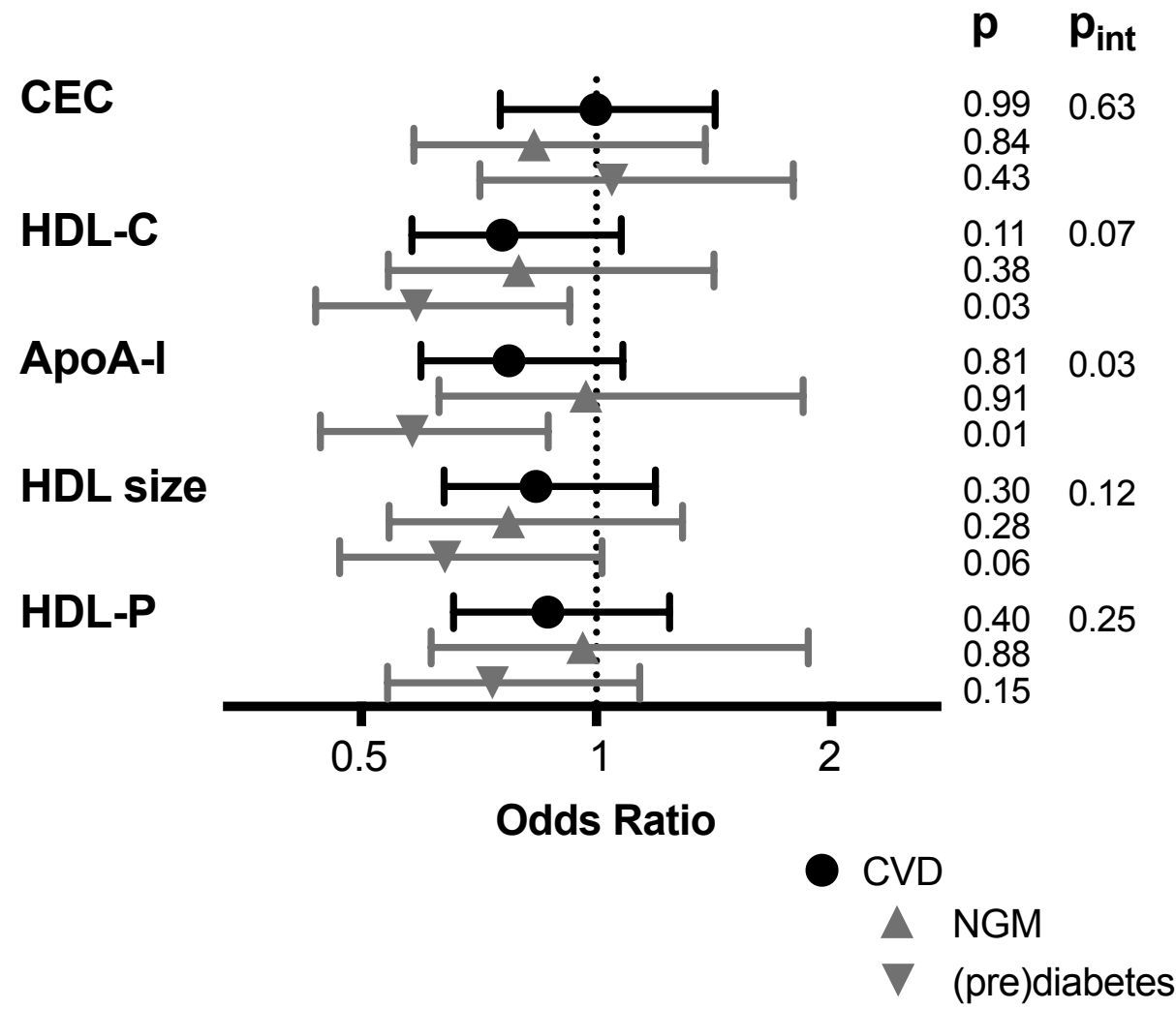

$\underline{1 b}$

\section{CVE}

CEC

HDL-C

ApoA-1

HDL size

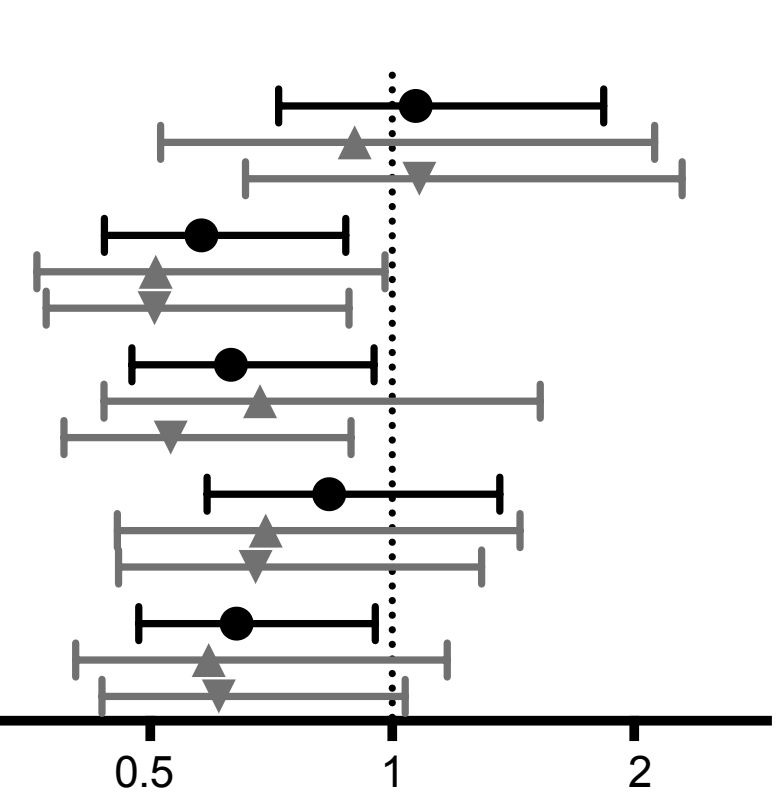

p $\mathbf{p}_{\text {int }}$

$\begin{array}{ll}0.77 & 0.91\end{array}$

0.76

0.80

$0.01 \quad 0.17$

0.05

0.02

$0.03 \quad 0.13$

0.29

0.02

$0.42 \quad 0.30$

0.27

0.20

$0.03 \quad 0.54$

HDL-P

0.11

0.06

Odds Ratio

- CVE

$\triangle$ NGM

$\nabla$ (pre)diabetes 
Effect of glucose tolerance status on the associations between HDL characteristics and prevalent cardiovascular outcomes

Interaction analyses suggested that the strength of the association of CEC with CIMT $\left(p_{\text {interaction }}=0.025\right)$ and the EnD Score $\left(p_{\text {interaction }}=0.103\right)$ might differ between those with NGM and those with (pre)diabetes. Stratified analyses showed that CEC was not significantly associated with cIMT (Table 3) or the EnD Score (Table 4) in either of the subgroups (cIMT: NGM $\beta=0.06[-0.04 ; 0.17] p=0.25$, (pre)diabetes $\beta=-0.13[-0.26$; 0.01 ] $p=0.060$; EnD Score: $N G M \quad \beta=-0.08$ [-0.18; 0.03] $p=0.142$, (pre)diabetes $\beta=0.06$ [ $-0.06 ; 0.19], p=0.312$; full model). Potential interaction was also observed for the strength of the association of apoA-I ( $\left.p_{\text {interaction }}=0.067\right)$ and HDL-P $\left(p_{\text {interaction }}=0.038\right)$ with cIMT but no significant associations were observed within the subgroups (NGM or (pre)diabetes, Table 3). The strength of the associations of HDL-size and HDL-P with the EnD Score also appeared to differ between NGM and (pre)diabetes (pinteraction $=0.039$ and 0.006 , respectively). HDL-size and HDL-P were inversely associated with the EnD Score in those with NGM, while such association was not observed in individuals with (pre)diabetes (Table 4). Notably, a significant inverse association between HDL-C and the EnD Score was observed in NGM as in (pre)diabetes ( $p_{\text {interaction }}=0.508$ ). Additional adjustments for plasma TG and LDL-C levels did not essentially change the strength of these associations (Suppl. Tables S4 and S5).

Table 3: Linear regression analyses, Model 1: crude, Model 2: adjusted for age, sex, Model 3 additionally adjusted for smoking, bmi, medication usage (antihypertensive, glucose- and lipid lowering), physical activity, caloric intake, systolic and diastolic blood pressure, fasting plasma glucose, and glucose metabolism state. Std $\beta$; standardized regression coefficient.

Table 4: Linear regression analyses, Model 1: crude, Model 2: adjusted for age, sex, Model 3 additionally adjusted for smoking, bmi, medication usage (antihypertensive, glucose- and lipid lowering), physical activity, caloric intake, systolic and diastolic blood pressure, fasting plasma glucose, and glucose metabolism state. Std $\beta$; standardized regression coefficient. 
Table 3. Relationship of HDL-CEC and other HDL characteristics with cIMT, stratified for normoglycemia and (pre)diabetes.

\begin{tabular}{|c|c|c|c|c|c|c|c|c|}
\hline \multirow{3}{*}{\multicolumn{2}{|c|}{ odel }} & \multicolumn{6}{|c|}{ cIMT (Z-Score) } & \multirow{3}{*}{ Pint } \\
\hline & & \multicolumn{3}{|c|}{$\begin{array}{r}\text { NGM } \\
N=268\end{array}$} & \multicolumn{3}{|c|}{$\begin{array}{l}\text { IGM\&T2DM } \\
\mathrm{N}=228\end{array}$} & \\
\hline & & std $\beta$ & $95 \% \mathrm{Cl}$ & p-value & std $\beta$ & $95 \% \mathrm{Cl}$ & p-value & \\
\hline \multirow[t]{3}{*}{ HDL-CEC } & 1 & 0.062 & $-0.056 ; 0.179$ & 0.303 & -0.152 & $-0.286 ;-0.017$ & 0.028 & 0.019 \\
\hline & 2 & 0.059 & $-0.052 ; 0.170$ & 0.296 & -0.132 & $-0.265 ; 0.001$ & 0.051 & 0.023 \\
\hline & 3 & 0.061 & $-0.044 ; 0.167$ & 0.255 & -0.129 & $-0.263 ; 0.005$ & 0.060 & 0.025 \\
\hline \multirow[t]{3}{*}{ HDL-C } & 1 & -0.057 & $-0.172 ; 0.058$ & 0.328 & -0.181 & $-0.325 ;-0.038$ & 0.014 & 0.184 \\
\hline & 2 & -0.035 & $-0.150 ; 0.079$ & 0.545 & -0.138 & $-0.287 ; 0.012$ & 0.071 & 0.216 \\
\hline & 3 & -0.044 & $-0.158 ; 0.070$ & 0.446 & -0.138 & $-0.293 ; 0.017$ & 0.081 & 0.169 \\
\hline \multirow[t]{3}{*}{ ApoA-I } & 1 & 0.007 & $-0.111 ; 0.125$ & 0.905 & -0.188 & $-0.325 ;-0.025$ & 0.007 & 0.033 \\
\hline & 2 & 0.031 & $-0.088 ; 0.149$ & 0.611 & -0.132 & $-0.277 ; 0.014$ & 0.076 & 0.075 \\
\hline & 3 & 0.011 & $-0.105 ; 0.128$ & 0.848 & -0.118 & $-0.268 ; 0.031$ & 0.120 & 0.067 \\
\hline \multirow[t]{3}{*}{ HDL size } & 1 & -0.088 & $-0.196 ; 0.021$ & 0.114 & -0.056 & $-0.207 ; 0.095$ & 0.465 & 0.737 \\
\hline & 2 & -0.061 & $-0.172 ; 0.050$ & 0.279 & -0.009 & $-0.165 ; 0.147$ & 0.908 & 0.823 \\
\hline & 3 & -0.055 & $-0.167 ; 0.056$ & 0.329 & -0.006 & $-0.170 ; 0.159$ & 0.947 & 0.910 \\
\hline \multirow[t]{3}{*}{ HDL-P } & 1 & -0.007 & $-0.130 ; 0.115$ & 0.909 & -0.169 & $-0.298 ;-0.039$ & 0.011 & 0.061 \\
\hline & 2 & 0.043 & $-0.081 ; 0.167$ & 0.496 & -0.112 & $-0.253 ; 0.029$ & 0.118 & 0.080 \\
\hline & 3 & 0.006 & $-0.115 ; 0.128$ & 0.919 & -0.132 & $-0.276 ; 0.012$ & 0.073 & 0.038 \\
\hline
\end{tabular}

Table 4. Relationship of HDL-CEC and other HDL characteristics with the EnD Score, stratified for normoglycemia and (pre)diabetes.

\begin{tabular}{|c|c|c|c|c|c|c|c|c|}
\hline & \multirow[b]{3}{*}{ Model } & \multicolumn{7}{|c|}{ EnD (Z-Score) } \\
\hline & & \multicolumn{3}{|c|}{ NGM (N=279) } & \multicolumn{3}{|c|}{ IGM \& T2DM (N=254) } & \multirow[b]{2}{*}{ Pint } \\
\hline & & std $\beta$ & $95 \% \mathrm{Cl}$ & $p$-value & $\operatorname{std} \beta$ & $95 \% \mathrm{Cl}$ & $p$-value & \\
\hline \multirow[t]{3}{*}{ HDL-CEC } & 1 & -0.079 & $-0.186 ; 0.028$ & 0.146 & 0.039 & $-0.094 ; 0.172$ & 0.564 & 0.170 \\
\hline & 2 & -0.105 & $-0.210 ;-0.001$ & 0.047 & 0.039 & $-0.093 ; 0.171$ & 0.562 & 0.099 \\
\hline & 3 & -0.076 & $-0.177 ; 0.025$ & 0.142 & 0.064 & $-0.060 ; 0.188$ & 0.312 & 0.211 \\
\hline \multirow[t]{3}{*}{ HDL-C } & 1 & -0.269 & $-0.370 ;-0.168$ & $<0.001$ & -0.242 & $-0.383 ;-0.101$ & 0.001 & 0.755 \\
\hline & 2 & -0.330 & $-0.432 ;-0.228$ & $<0.001$ & -0.278 & $-0.424 ;-0.132$ & $<0.001$ & 0.695 \\
\hline & 3 & -0.256 & $-0.362 ;-0.150$ & $<0.001$ & -0.185 & $-0.330 ;-0.040$ & 0.013 & 0.508 \\
\hline \multirow[t]{3}{*}{ ApoA-I } & 1 & -0.216 & $-0.321 ;-0.111$ & $<0.001$ & -0.125 & $-0.160 ; 0.010$ & 0.069 & 0.290 \\
\hline & 2 & -0.288 & $-0.394 ;-0.181$ & $<0.001$ & -0.144 & $-0.287 ;-0.001$ & 0.049 & 0.162 \\
\hline & 3 & -0.225 & $-0.333 ;-0.117$ & $<0.001$ & -0.071 & $-0.210 ; 0.067$ & 0.312 & 0.102 \\
\hline \multirow[t]{3}{*}{ HDL size } & 1 & -0.212 & $-0.310 ;-0.114$ & $<0.001$ & -0.047 & $-0.195 ; 0.100$ & 0.528 & 0.062 \\
\hline & 2 & -0.277 & $-0.378 ;-0.176$ & $<0.001$ & -0.077 & $-0.231 ; 0.078$ & 0.331 & 0.068 \\
\hline & 3 & -0.204 & $-0.309 ;-0.099$ & $<0.001$ & 0.03 & $-0.119 ; 0.185$ & 0.668 & 0.039 \\
\hline \multirow[t]{3}{*}{ HDL-P } & 1 & -0.193 & $-0.304 ;-0.083$ & 0.001 & 0.038 & $-0.091 ; 0.166$ & 0.564 & 0.005 \\
\hline & 2 & -0.252 & $-0.366 ;-0.138$ & $<0.001$ & 0.045 & $-0.093 ; 0.182$ & 0.523 & 0.002 \\
\hline & 3 & -0.205 & $-0.319 ;-0.092$ & $<0.001$ & 0.037 & $-0.095 ; 0.168$ & 0.582 & 0.006 \\
\hline
\end{tabular}


HDL-CEC was not associated with CVD in either NGM or (pre)diabetes (Figure 1, panel a). We did observe a difference in the strength of the association of HDL-C and apoA-I with CVD ( interaction $=0.074$ and 0.034 , respectively). In individuals with NGM ( $\mathrm{N}=67 \mathrm{CVD}$ cases) no association of HDL-C or apoA-I with CVD was observed (HDL-C: OR $=0.84$ [0.56; 1.25] $p=0.38$, apoA-I: OR 0.98 [0.67; 1.44] $p=0.91$ ), while in those with (pre)diabetes ( $\mathrm{N}=83 \mathrm{CVD}$ cases), an increase of one unit SD of HDL-C or apoA-I was associated with 1.5-fold fewer cases (HDL-C: OR=0.62 [0.40; 0.94] $p=0.026$; apoA-I: OR=0.61 [0.41;0.90] $p=0.012$ ). Adjusting for LDL-C did not change these associations, but adjustment for TG attenuated the strength of association between HDL-C and CVD (Suppl. Table 6). No significant interaction with glucose metabolism status was observed for the associations with CVE (pinteraction between 0.13 and 0.98) but for completeness the stratified analyses are included in Figure1, panel b. Notably, a significant inverse association between HDL-C and CVE was observed in those with NGM as well as those with (pre)diabetes ( $p_{\text {interaction }}=0.171$ ).

\section{Discussion}

In this observational human cohort, we show that HDL-CEC is not associated with parameters of atherosclerosis as reflected by cIMT and the EnD Score, nor with prevalent CVD or CVE, independent of glucose metabolism state. In contrast, other HDL characteristics, i.e. HDL-C, apoA-I, HDL size and HDL-P, were inversely associated with the EnD Score and with CVE. The inverse association between HDL-C and the EnD Score was observed in subjects with NGM, as well as those with (pre)diabetes, while the inverse associations with apoA-I, HDL size and HDL-P were found only in individuals with NGM. On the other hand, higher concentrations of HDL-C and apoA-I were associated with less CVD in individuals with (pre)diabetes, but not in those with NGM.

The role of HDL in atherosclerosis has undergone a major re-evaluation in the past 5 years, leading to intensified research on HDL-CEC ${ }^{44}$. Thus far, the results of these efforts are not uniform and the final conclusion on whether and how HDL-CEC impacts atherosclerosis and CVD/CVE is yet to be made. Some studies showed that an increase in HDL-CEC could be beneficial for atherosclerosis ${ }^{16}$ as well as CVD/ CVE $^{17,18}$, while others reported the opposite ${ }^{14,15,20}$. In our current evaluation, we did not find any significant associations for HDL-CEC, which is in line with prevalent ${ }^{20,45}$ 
and incident studies. ${ }^{46} \mathrm{Li}$ et $\mathrm{a}^{20}$ reported that there was no association of HDL-CEC with prevalent CV risk in a cohort with stable angiographically confirmed CVD after adjustment for traditional risk factors, such as age, sex, smoking, diabetes, hypertension, LDL-C, and HDL-C. However, in the same cohort, they unexpectedly observed an association between higher HDL-CEC and increased incident CV events, suggesting a complex relationship between HDL-CEC and vascular disease. The relation of HDL-CEC might differ between atherosclerotic plaque development (=prevalent CVD) and plaque vulnerability-associated phenotypes e.g. myocardial infarction (=incident CVD). Further, a recent study showed that the association of cholesterol mass efflux capacity differs between coronary heart disease and stroke. ${ }^{19}$

In contrast to our null-findings for HDL-CEC, other HDL characteristics, i.e. HDL-C, apoA-I, HDL size and HDL-P, were inversely associated with the EnD Score, CVD and CVE. These HDL-characteristics were not associated with CIMT in our current evaluations, although the direction of the non-significant associations with cIMT were also inverse.

In the CODAM cohort, as in several other studies ${ }^{47-49}$, we observed an inverse association of HDL-C with the EnD Score and CVE, which was independent of LDL-C. Despite these robust inverse associations of HDL-C with risk of atherosclerosis/CVE, pharmacological elevation of $\mathrm{HDL}-\mathrm{C}$ has failed to reduce atherosclerosis ${ }^{50}$ or cardiac events. ${ }^{3,4}$ For this reason, the attention has shifted not only from HDL-C to HDL-CEC, but also to further alternative indexes of $\mathrm{HDL}$ quantity and/or quality such as apoA-I, HDL size and HDL-P. ${ }^{51}$ As expected, we found inverse associations of apoA-I with atherosclerosis and CVD/CVE. ApoA-I is the main protein component $(70 \%)$ of $\mathrm{HDL}$. Several ways of atheroprotective mechanisms for apoA-I have been proposed and pharmacological interventions targeting apoA-I are in process (recently reviewed $i^{51,52}$ ). Also in line with our results is the current literature on HDL-P, which consistently reports an inverse association of HDL-P with atherosclerosis ${ }^{53}, \mathrm{CVE}^{54}$, future CAD risk ${ }^{53,55}$, as well as a lower rate of coronary heart disease (CHD) death. ${ }^{56}$ In our current evaluation, the association of HDL-P with the EnD Score and CVE was slightly attenuated after adjustment for TG, thereby remaining significant for CVE, but not for $\mathrm{EnD}$ Score. The inverse association of HDL size with the $\mathrm{EnD}$ Score was more strongly attenuated by adjusting for TG. These findings are consistent with the report of Harchaoui et al. ${ }^{55}$, who showed that the relationship between HDL-size and CAD risk 
was dependent on metabolic parameters, while the relationship of HDL-P with CAD was not.

Overall, HDL is a heterogeneous lipoprotein fraction encompassing particles that markedly differ in size, density, surface charge and composition. ${ }^{57}$ It exerts several atheroprotective properties, such as, anti-apoptotic, anti-oxidant and nitric oxide promoting effects. However, which atheroprotective property is the most clinically relevant one, and what subfraction of HDL exerts which property, remains unknown. Notably, the metabolic milieu may affect various properties of HDL. Therefore, we investigated whether the observed associations differed between those with normoglycemia (NGM) and those with (pre)diabetes. The inverse associations of HDL-P and HDL-size with the EnD Score in subjects with NGM were fully absent in those with (pre)diabetes. In contrast, the inverse association for HDL-C was clearly present in both NGM and (pre)diabetes. Our study population displays the typical dyslipidemic phenotype, i.e. high TG and low HDL-C, but not the decreased HDL-size and lower HDL-P, that has been shown before ${ }^{58,59}$. This suggests that in those with NGM, the protective effect of a certain number of HDL particles against development of $\mathrm{EnD}$ is better than for that same number of HDL particles in (pre)diabetes. Thus, HDL particle composition, perhaps partly reflected in HDL size, is of interest regarding its protective vascular properties. In this context, a possible compromised antioxidative capability of HDL, may be of importance. ${ }^{60}$ It has been shown before that HDL is able to reduce cytokine-induced expression of VCAM-1, ICAM-1, and E-Selectin in vitro ${ }^{61}$ and in vivo ${ }^{62}$. The observation that the inverse associations of HDL size and HDL-P are lost in (pre)diabetes subjects, suggests the loss of their antiinflammatory and endothelium-protective properties in people with impaired glucose metabolism and insulin resistance. In contrast to our observations on the EnD Score, the association of higher concentrations of HDL-C and apoA-I with less CVD was only seen in individuals with (pre)diabetes. This was an unexpected observation that to some extent may be explained by more careful monitoring by health care professionals of individuals with (pre)diabetes for signs of development of CVD.

The main strength of this study is the relatively large population, the combination of several HDL characteristics, the availability of the EnD Score and cIMT in addition to CVD/CVE, and the approximately equal numbers of individuals with NGM and (pre)diabetes, which allowed us to perform sufficiently numbered stratified analyses. 
Further, we are the first to report on the relationship of CEC with CVD/CVE in individuals with (pre)diabetes. The interpretation of our current study is limited by the fact that we measured only one functional aspect of HDL function, i.e. its CEC, which, is thought to reflect a key cardioprotective HDL functionality. We employed a wellvalidated HDL-CEC assay using human THP-1 cell-derived foam cells as cholesterol donor and apoB-depleted plasma as cholesterol acceptor. Significant positive associations of HDL-CEC with other HDL characteristics (HDL-C, apoA-I, HDL size, HDL-P) substantiate its measurement (Supplemental Figure 1). However, so far, a gold standard to measure HDL-CEC in humans does not exist and the optimal method for HDL isolation remains undecided, likely leading to discrepancies between studies. ${ }^{63}$ The Rader Assay, another HDL-CEC method using apoB depleted plasma on murine J774 cells, has been shown to correlate with CVD. ${ }^{17}$ Therefore, we remeasured HDL-CEC in a subset of 57 CODAM samples using the Rader Assay and observed a significant correlation with an intraclass correlation coefficient of 0.6 (data not shown). In our opinion, employing human THP-1 monocyte-derived macrophage foam cells for HDL-CEC measurements provides a closer approximation of the condition in human atherosclerotic plaques, due to the use of a homologous system (human cells and human apoB-depleted plasma) and the expression of all relevant efflux transporters (ATP-binding cassette transporter A1 (ABCA1), ABCG1 and scavenger receptor B1 $\left(\right.$ SR-B1)). ${ }^{41}$

Further, the associations of HDL characteristics with the two surrogate markers of atherosclerosis, EnD Score and cIMT, were not always fully concordant. This may partly be due to the fact that they represent different aspects of atherosclerosis and partly to the measurements themselves. CIMT is a widely used noninvasive measure of atherosclerosis ${ }^{64}$ and has been identified as a reliable indicator of generalized atherosclerosis. ${ }^{65}$ However, cIMT measurements are not standardized ${ }^{66,67}$ and the sonographer, image analyst as well as random error can introduce variability. ${ }^{68}$ Therefore, the difference in association of these parameters with cIMT and the EnD Score may be due to the sensitivity and variability of the cIMT measurements itself. Further, the washout period for lipid-lowering therapy was 14 days and may not have been sufficient to eliminate all their metabolic effects. However, the CODAM study includes participants with pre-existing CVD, thus 14 days were considered the maximum period for safety reasons. For the extrapolation of our findings, it should be 
considered that our study includes individuals with at least one cardiometabolic risk factors. Nonetheless, the cohort represents a large part of typical Western populations, namely middle-aged to elderly individuals with a moderately increased risk of cardiometabolic disease. Lastly, the cross-sectional approach of our study hampers a direct causal interpretation of the observed associations.

\section{Conclusion}

In summary, in a cohort of 533 patients with an elevated risk of CVD and T2DM, we observed that HDL-CEC was not associated with atherosclerosis or CVD/CVE, neither in the whole population nor in individuals with (pre)diabetes, while other HDL characteristics showed atheroprotective associations. Notably, the atheroprotective associations of HDL-size and HDL-P are lost in individuals with (pre)diabetes. The fact that, particularly in T2DM, higher apoA-I and HDL-C concentrations were related to less CVD in subjects with (pre)diabetes, independent of CVD risk factors, was an unexpected observation and might be related to more intense cardiovascular risk monitoring and awareness of those individuals.

\section{Conflict of Interest:}

The authors declare that they have no conflict of interest.

\section{Contributions:}

T.J. and M.M.J.VG performed the analyses and T.J. created the Figures and Tables. T.J., K.W. and M.M.J.vG wrote and edited the manuscript. U.J.F.T., W.A., R.P.F.D., T.V. performed HDL-CEC assays, I.C.W.A. contributed to the lipoprotein measurements, M.M.J.vG., C.J.H.K., C.D.A.S., C.G.S. are part of the CODAM steering committee, E.A.F and I.J.G were involved in setting up the study. All co-authors read and edited the manuscript.

\section{Acknowledgements:}

none 


\section{Sources of Funding:}

The Netherlands Organization for Scientific Research (940-35-034; CODAM study), Dutch Diabetes Research Foundation (98.901; CODAM study). AHA Predoctoral Fellowship (18PRE33990436; TJ), NIH grants (HL129433; HL092969; 123398; EAF, IJG, TJ). Part of the measurements were supported through grants from The Maastricht University Medical Center+ and the Biobanking and Biomolecular Resources Research Infrastructure (BBMRI) Metabolomics Consortium funded by BBMRI-NL, a research infrastructure financed by the Dutch government (NWO, 184.021.007 and 184033111). 


\section{References}

1. Rosenson, R. S. et al. Dysfunctional HDL and atherosclerotic cardiovascular disease. Nat. Rev. Cardiol. 13, 48-60 (2016).

2. Badimon, J. J. \& Santos-Gallego, C. G. HDL Dysfunction Is the Answer in the Sphinx's Riddle? J. Am. Coll. Cardiol. 66, 1486-1488 (2015).

3. Barter, P. J. et al. Effects of Torcetrapib in Patients at High Risk for Coronary Events. N. Engl. J. Med. 357, 2109-2122 (2017).

4. HPS2-THRIVE Collaborative Group. Effects of Extended-Release Niacin with Laropiprant in High-Risk Patients. N. Engl. J. Med. 371, 203-212 (2014).

5. Glomset, J. A. The plasma lecithin:cholesterol acyltransferase reaction. J. Lipid Res. 9, 155-167 (1968).

6. Santos-Gallego, C. G., Badimon, J. J. \& Rosenson, R. S. Beginning to understand high-density lipoproteins. Endocrinol. Metab. Clin. North Am. 43, 913-947 (2014).

7. Rader, D. J., Alexander, E. T., Weibel, G. L., Billheimer, J. \& Rothblat, G. H. The role of reverse cholesterol transport in animals and humans and relationship to atherosclerosis. J. Lipid Res. 50, S189-S194 (2009).

8. Tall, A. R., Yvan-Charvet, L., Terasaka, N., Pagler, T. \& Wang, N. HDL, ABC Transporters, and Cholesterol Efflux: Implications for the Treatment of Atherosclerosis. Cell Metab. 7, 365-375 (2008).

9. Out, R. et al. Combined deletion of macrophage ABCA1 and ABCG1 leads to massive lipid accumulation in tissue macrophages and distinct atherosclerosis at relatively low plasma cholesterol levels. Arterioscler. Thromb. Vasc. Biol. 28, 258-264 (2008).

10. Yvan-Charvet, L. et al. Combined deficiency of ABCA1 and ABCG1 promotes foam cell accumulation and accelerates atherosclerosis in mice. J. Clin. Invest. 117, 3900-3908 (2007).

11. Feig, J. E. et al. HDL promotes rapid atherosclerosis regression in mice and alters inflammatory properties of plaque monocyte-derived cells. Proc. Natl. Acad. Sci. 108, 7166-7171 (2011).

12. Hewing, B. et al. Effects of native and myeloperoxidase-modified apolipoprotein A-I on reverse cholesterol transport and atherosclerosis in mice. Arterioscler. Thromb. Vasc. Biol. 34, 779-789 (2014).

13. Tang, C., Houston, B. A., Storey, C. \& Leboeuf, R. C. Both STAT3 activation and cholesterol efflux contribute to the anti-inflammatory effect of apoAI/ABCA1 interaction in macrophages. J. Lipid Res. 57, 848-857 (2016).

14. Smoak, K. A. et al. Myeloid differentiation primary response protein 88 couples reverse cholesterol transport to inflammation. Cell Metab. 11, 493-502 (2010).

15. van der Vorst, E. P. C. et al. High-Density Lipoproteins Exert Pro-inflammatory Effects on Macrophages via Passive Cholesterol Depletion and PKC-NFKB/STAT1-IRF1 Signaling. Cell Metab. 25, 197-207 (2017).

16. Khera, A. V. et al. Cholesterol Efflux Capacity, High-Density Lipoprotein Function, and Atherosclerosis. N. Engl. J. Med. 364, 127-135 (2011).

17. Rohatgi, A. et al. HDL Cholesterol Efflux Capacity and Incident Cardiovascular Events. N. Engl. J. Med. 371, 2383-2393 (2014).

18. Saleheen, D. et al. Association of HDL cholesterol efflux capacity with incident coronary heart disease events: A prospective case-control study. Lancet Diabetes Endocrinol. 3, 507-513 (2015).

19. Shea, S. et al. Cholesterol Mass Efflux Capacity, Incident Cardiovascular 
Disease, and Progression of Carotid Plaque The Multi-Ethnic Study of Atherosclerosis. Arterioscler. Thromb. Vasc. Biol. 39, 89-96 (2019).

20. Li, X. M. et al. Paradoxical association of enhanced cholesterol efflux with increased incident cardiovascular risks. Arterioscler. Thromb. Vasc. Biol. 33, 1696-1705 (2013).

21. Sarwar, N. et al. Diabetes mellitus, fasting blood glucose concentration, and risk of vascular disease: A collaborative meta-analysis of 102 prospective studies. Lancet 375, 2215-2222 (2010).

22. Kubota, M. et al. Relationship between serum cholesterol efflux capacity and glucose intolerance in Japanese-Americans. J. Atheroscler. Thromb. 21, 10871097 (2014).

23. Apro, J. et al. Impaired cholesterol efflux capacity of high-density lipoprotein isolated from interstitial fluid in type 2 diabetes mellitus - brief report.

Arterioscler. Thromb. Vasc. Biol. 36, 787-791 (2016).

24. Annema, W. et al. Impaired HDL cholesterol efflux in metabolic syndrome is unrelated to glucose tolerance status: The CODAM study. Sci. Rep. 6, 1-9 (2016).

25. Low, H. et al. Advanced glycation end-products (AGEs) and functionality of reverse cholesterol transport in patients with type 2 diabetes and in mouse models. Diabetologia 55, 2513-2521 (2012).

26. Dullaart, R. P. F., Annema, W., de Boer, J. F. \& Tietge, U. J. F. Pancreatic $\beta-$ cell function relates positively to HDL functionality in well-controlled Type 2 diabetes mellitus. Atherosclerosis 222, 567-573 (2012).

27. de Vries, R. et al. Increased cholesterol efflux from cultured fibroblasts to plasma from hypertriglyceridemic type 2 diabetic patients: Roles of pre $\beta-H D L$, phospholipid transfer protein and cholesterol esterification. Atherosclerosis 196, 733-741 (2008).

28. Yassine, H. N. et al. Enhanced cholesterol efflux to HDL through the ABCA1 transporter in hypertriglyceridemia of type 2 diabetes. Metabolism. 63, 727-734 (2014).

29. Nestel, P., Hoang, A., Sviridov, D. \& Straznicky, N. Cholesterol efflux from macrophages is influenced differentially by plasmas from overweight insulinsensitive and-resistant subjects. Int. J. Obes. 36, 407-413 (2012).

30. Chamaria, S. et al. Intracoronary Imaging, Cholesterol Efflux, and Transcriptomics after Intensive Statin Treatment in Diabetes. Sci. Rep. 7, 1-13 (2017).

31. Pia Pajunen, Mikko Syvänne, Graciel. Cholesterol Efflux Capacity In Vitro Predicts the Severity and Extent of Coronary Artery Disease in Patients with and without Type 2 Diabetes. Scand. Cardiovasc. J. 35, 96-100 (2001).

32. Jacobs, M. et al. Low-grade inflammation can partly explain the association between the metabolic syndrome and either coronary artery disease or severity of peripheral arterial disease: the CODAM study. Eur. J. Clin. Invest. 39, 43744 (2009).

33. Du, H. et al. Glycemic index and glycemic load in relation to food and nutrient intake and metabolic risk factors in a Dutch population. Am. J. Clin. Nutr. 87, 655-661 (2008).

34. Deckers, J. G., Schellevis, F. G. \& Fleming, D. M. WHO diagnostic criteria as a validation tool for the diagnosis of diabetes mellitus: A study in five European countries. Eur J Gen Pr. 12, 108-113 (2006). 
35. Engelen, L. et al. The association between the-374T/A polymorphism of the receptor for advanced glycation endproducts gene and blood pressure and arterial stiffness is modified by glucose metabolism status: The Hoorn and CoDAM studies. J. Hypertens. 28, 285-293 (2010).

36. van Bussel, B. C. T. et al. Multiple Inflammatory Biomarker Detection in a Prospective Cohort Study: A Cross-Validation between Well-Established Single-Biomarker Techniques and an Electrochemiluminescense-Based MultiArray Platform. PLoS One 8, e58576 (2013).

37. Stam, F. et al. Impaired renal function is associated with markers of endothelial dysfunction and increased inflammatory activity. Nephrol. Dial. Transplant. 18, 892-898 (2003).

38. Würtz, P. et al. Quantitative Serum Nuclear Magnetic Resonance Metabolomics in Large-Scale Epidemiology: A Primer on -Omic Technologies. Am. J. Epidemiol. 186, 1084-1096 (2017).

39. Kappelle, P. J. W. H. et al. Increased LCAT activity and hyperglycaemia decrease the antioxidative functionality of HDL. Eur. J. Clin. Invest. 42, 487495 (2012).

40. Annema, W. et al. Myeloperoxidase and serum amyloid A contribute to impaired in vivo reverse cholesterol transport during the acute phase response but not group IIA secretory phospholipase A(2). J. Lipid Res. 51, 743-754 (2010).

41. Ebtehaj, S., Gruppen, E. G., Bakker, S. J. L., Dullaart, R. P. F. \& Tietge, U. J. F. HDL (High-Density Lipoprotein) Cholesterol Efflux Capacity Is Associated With Incident Cardiovascular Disease in the General Population. Arterioscler. Thromb. Vasc. Biol. 1874-1883 (2019). doi:10.1161/atvbaha.119.312645

42. van Greevenbroek, M. M. J. et al. The cross-sectional association between insulin resistance and circulating complement C3 is partly explained by plasma alanine aminotransferase, independent of central obesity and general inflammation (the CODAM study). Eur. J. Clin. Invest. 41, 372-379 (2011).

43. Wendel-Vos, G. C. W., Schuit, A. J., Saris, W. H. M. \& Kromhout, D. Reproducibility and relative validity of the short questionnaire to assess healthenhancing physical activity. J. Clin. Epidemiol. 56, 1163-1169 (2003).

44. Fisher, E. A., Feig, J. E., Hewing, B., Hazen, S. L. \& Smith, J. D. HDL Function, Dysfunction, and Reverse Cholesterol Transport. Arterioscler. Thromb. Vasc. Biol. 32, 2813-2820 (2012).

45. Mutharasan, R. K. et al. HDL efflux capacity, HDL particle size, and high-risk carotid atherosclerosis in a cohort of asymptomatic older adults: the Chicago Healthy Aging Study. J. Lipid Res. 58, 600-606 (2017).

46. De Vries et al. Cholesterol Efflux Capacity and Atherosclerosis. N Engl J Med. 1472-1475 (2011).

47. Assmann, G., Schulte, H., Von Eckardstein, A. \& Huang, Y. High-density lipoprotein cholesterol as a predictor of coronary heart disease risk. The PROCAM experience and pathophysiological implications for reverse cholesterol transport. Atherosclerosis 124, S11-S20 (1996).

48. Barter, P. et al. HDL Cholesterol, Very Low Levels of LDL Cholesterol, and Cardiovascular Events. N. Engl. J. Med. 357, 1301-1310 (2007).

49. Gordon, T., Castelli, W. P., Hjortland, M. C., Kannel, W. B. \& Dawber, T. R. High density lipoprotein as a protective factor against coronary heart disease. Am. J. Med. 62, 707-714 (1977). 
50. Nissen, S. E. et al. Effect of Torcetrapib on the Progression of Coronary Atherosclerosis. N. Engl. J. Med. 356, 1304-1316 (2007).

51. Kajani, S., Curley, S. \& McGillicuddy, F. C. Unravelling hdl-looking beyond the cholesterol surface to the quality within. Int. J. Mol. Sci. 19, 1-23 (2018).

52. Zakiev, E., Feng, M., Sukhorukov, V. \& Kontush, A. HDL-Targeting Therapeutics: Past, Present and Future. Curr. Pharm. Des. 23, (2017).

53. MacKey, R. H. et al. High-density lipoprotein cholesterol and particle concentrations, carotid atherosclerosis, and coronary events: MESA (MultiEthnic Study of Atherosclerosis). J. Am. Coll. Cardiol. 60, 508-516 (2012).

54. Parish, S. et al. Lipids and lipoproteins and risk of different vascular events in the MRC/BHF heart protection study. Circulation 125, 2469-2478 (2012).

55. Harchaoui, E. Article Annals of Internal Medicine High-Density Lipoprotein Particle Size and Concentration and. Ann. Intern. Med. 150, 84-93 (2009).

56. Kuller, L. H., Grandits, G., Cohen, J. D., Neaton, J. D. \& Prineas, R. Lipoprotein particles, insulin, adiponectin, C-reactive protein and risk of coronary heart disease among men with metabolic syndrome. Atherosclerosis 195, 122-128 (2007).

57. Rye, K.-A. \& Barter, P. J. Cardioprotective functions of HDL. J. Lipid Res. 55, 168-179 (2014).

58. Mora, S. et al. Lipoprotein particle size and concentration by nuclear magnetic resonance and incident type 2 diabetes in women. Diabetes 59, 1153-1160 (2010).

59. Festa, A. et al. Nuclear magnetic resonance lipoprotein abnormalities in prediabetic subjects in the insulin resistance atherosclerosis study. Circulation 111, 3465-3472 (2005).

60. Viktorinova, A. et al. Abnormalities in the relationship of paraoxonase 1 with HDL and apolipoprotein A1 and their possible connection to HDL dysfunctionality in type 2 diabetes. Diabetes Res. Clin. Pract. 140, 174-182 (2018).

61. Cockerill, G. W., Rye, K.-A., Gamble, J. R., Vadas, M. A. \& Barter, P. J. HighDensity Lipoproteins Inhibit Cytokine-Induced Expression of Endothelial Cell Adhesion Molecules. Arterioscler. Thromb. Vasc. Biol. 15, 1987-1994 (1995).

62. Dimayuga, P. et al. Reconstituted HDL containing human apolipoprotein A-1 reduces VCAM-1 expression and neointima formation following periadventitial cuff-induced carotid injury in apoE null mice. Biochem. Biophys. Res. Commun. 264, 465-468 (1999).

63. Triolo, M., Annema, W., Dullaart, R. P. \& Tietge, U. J. Assessing the functional properties of high-density lipoproteins: an emerging concept in cardiovascular research. Biomark. Med. 7, 457-472 (2013).

64. Bots, M. L., Evans, G. W., Tegeler, C. H. \& Meijer, R. Carotid Intima-media Thickness Measurements: Relations with Atherosclerosis, Risk of

Cardiovascular Disease and Application in Randomized Controlled Trials. Chin. Med. J. (Engl). 129, 215-226 (2016).

65. Iwakiri, T. et al. Usefulness of carotid intima-media thickness measurement as an indicator of generalized atherosclerosis: Findings from autopsy analysis. Atherosclerosis 225, 359-362 (2012).

66. Peters, S. A. E., Den Ruijter, H. M. \& Bots, M. L. Ultrasound protocols to measure carotid intima-media thickness: One size does not fit all. J. Am. Soc. Echocardiogr. 25, 1135-1137 (2012). 
67. den Ruijter, H. M. et al. Common Carotid Intima-Media Thickness

Measurements in Cardiovascular Risk Prediction: A Meta-Analysis. JAMA - J. Am. Med. Assoc. 308, 796-803 (2012).

68. De Groot, E. et al. Variance Components analysis of carotid and femoral intima-media thickness measurements. 24, 825-832 (1998). 


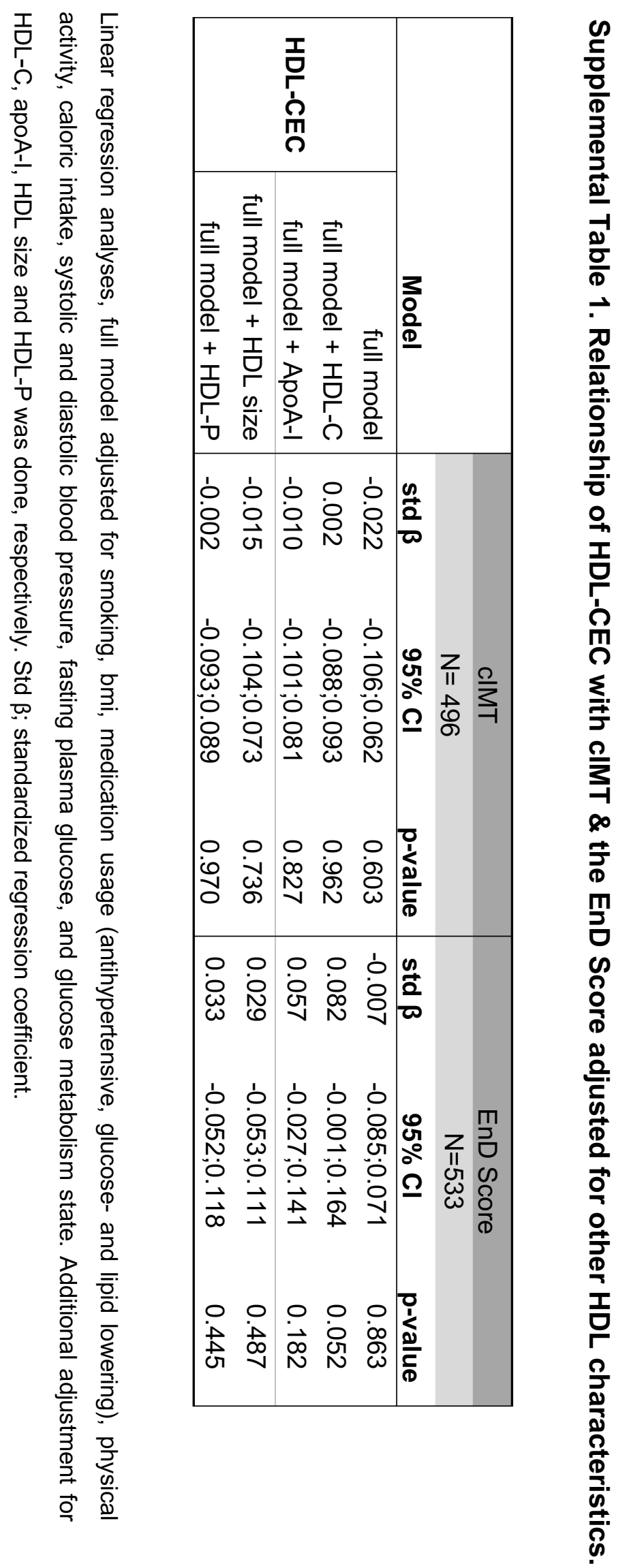




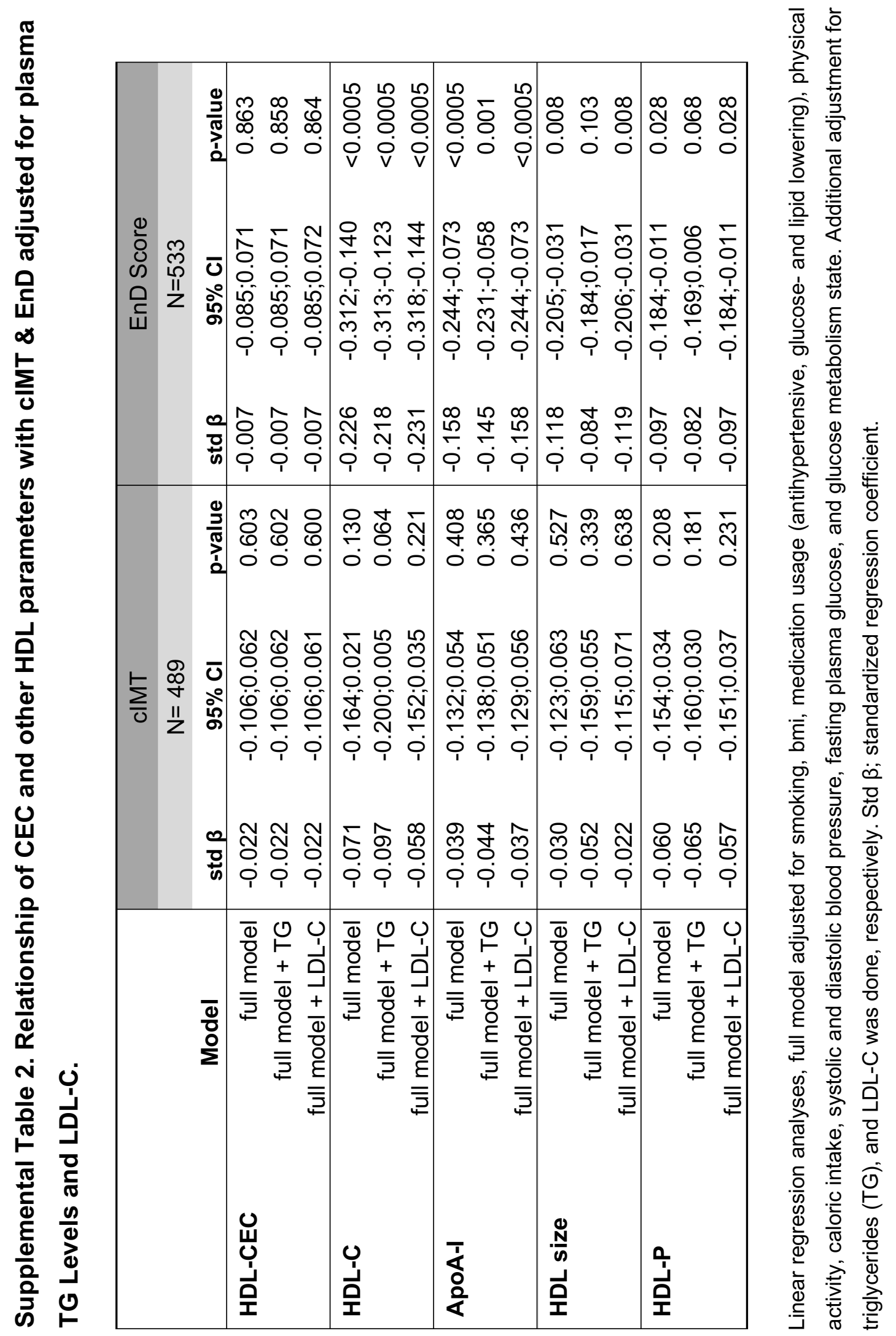




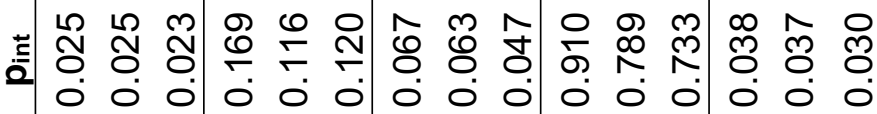

光

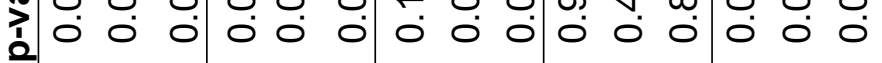

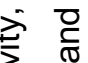

范

$\frac{\overline{0}}{\sqrt{0}}$

誉

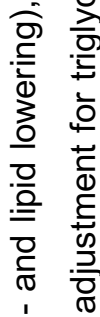

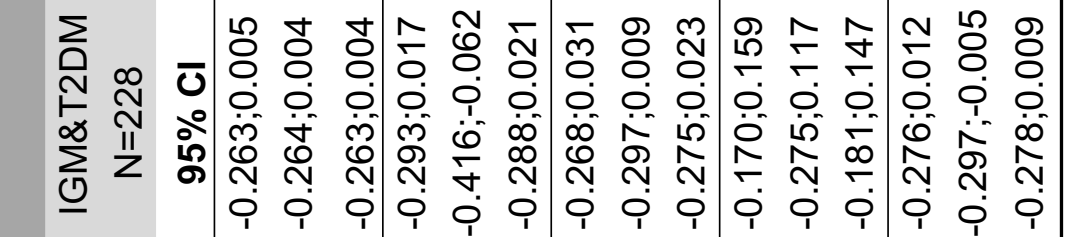

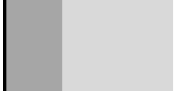

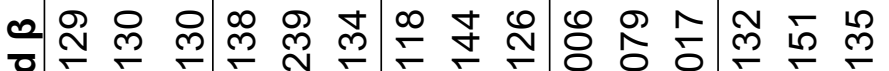
虫

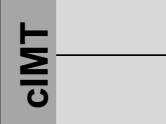

ᄂ N $\begin{array}{llllllllllllllll}0 & 0 & 0 & 0 & 0 & 0 & 0 & 0 & 0 & 0 & 0 & 0 & 0 & 0 & 0\end{array}$

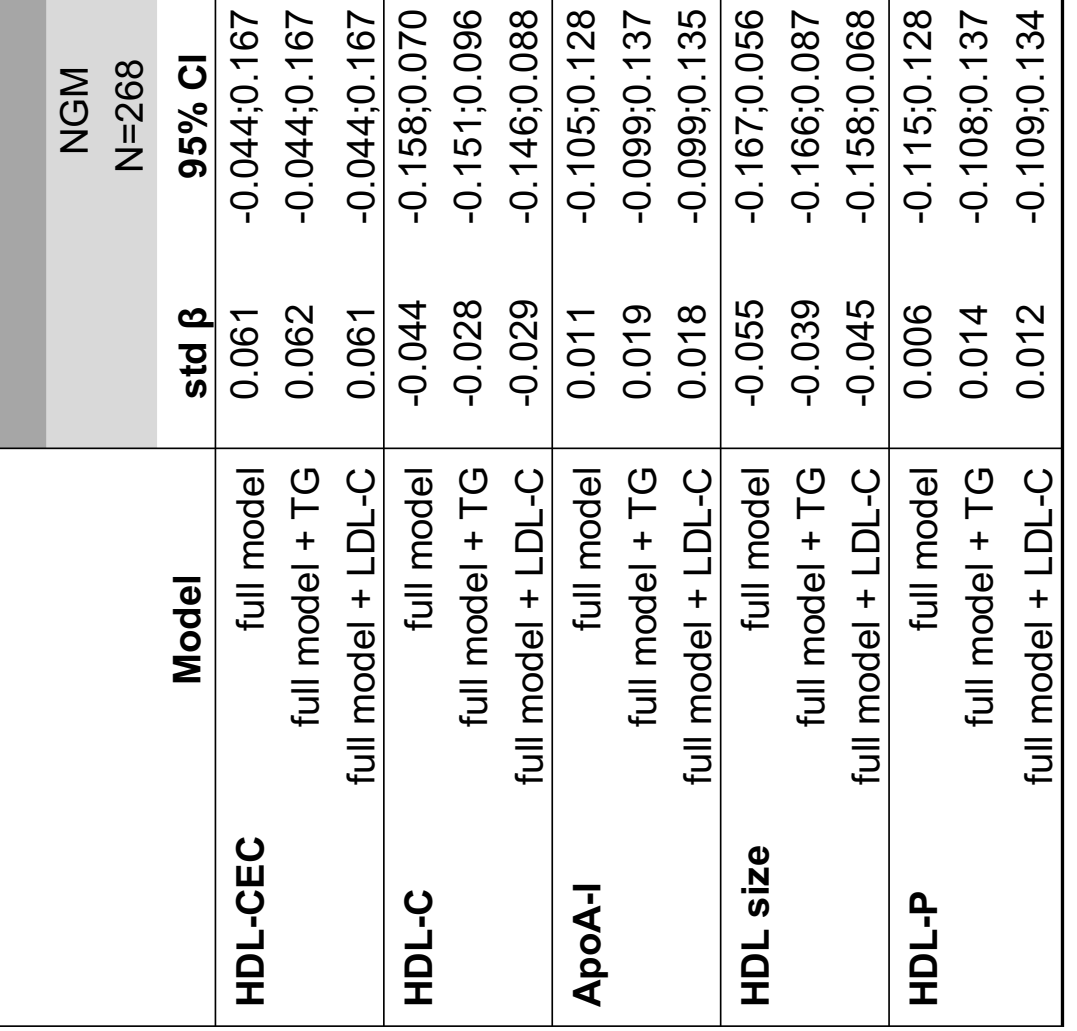

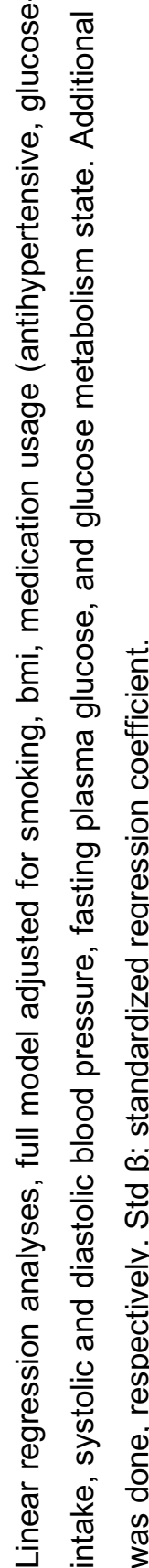




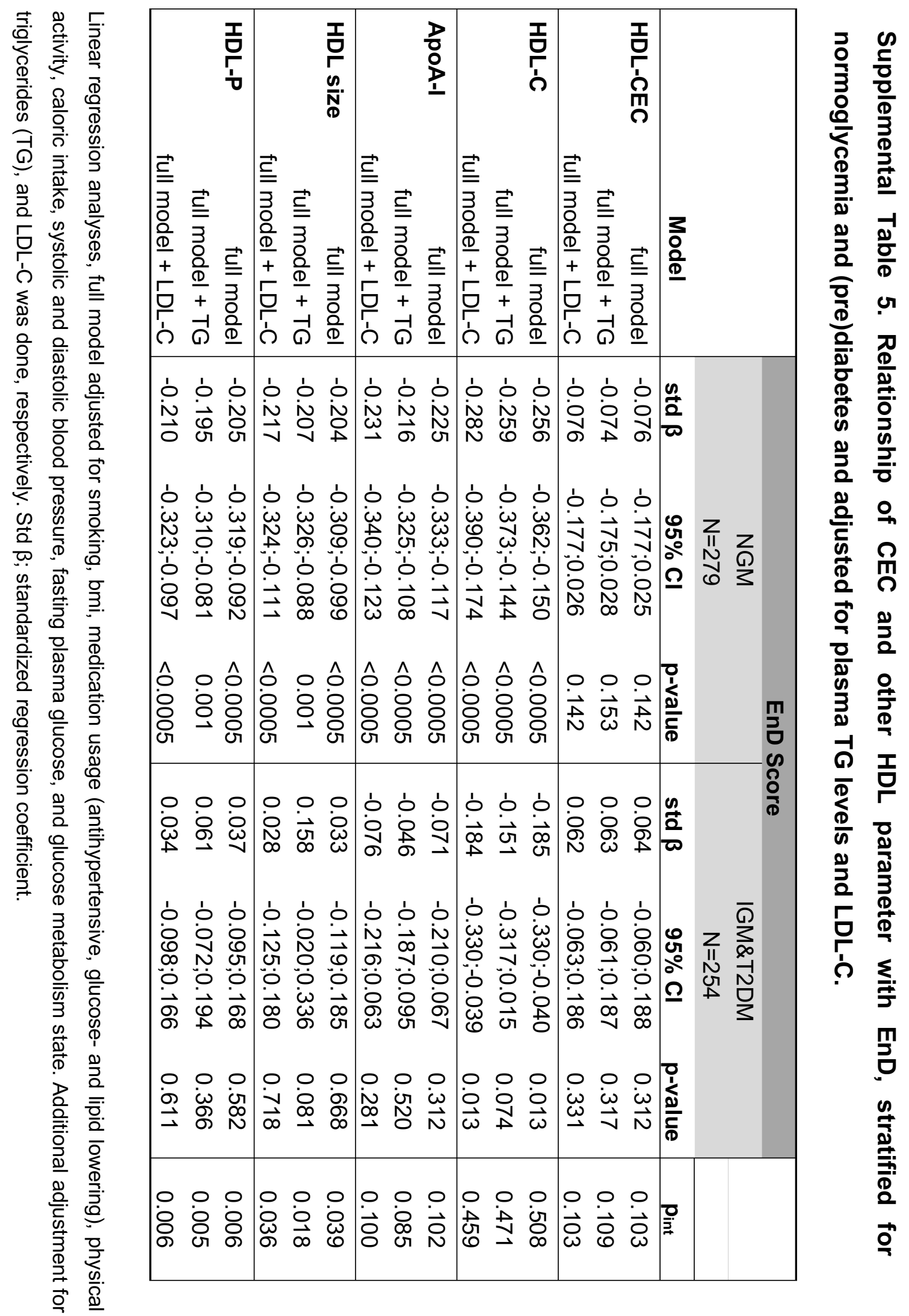




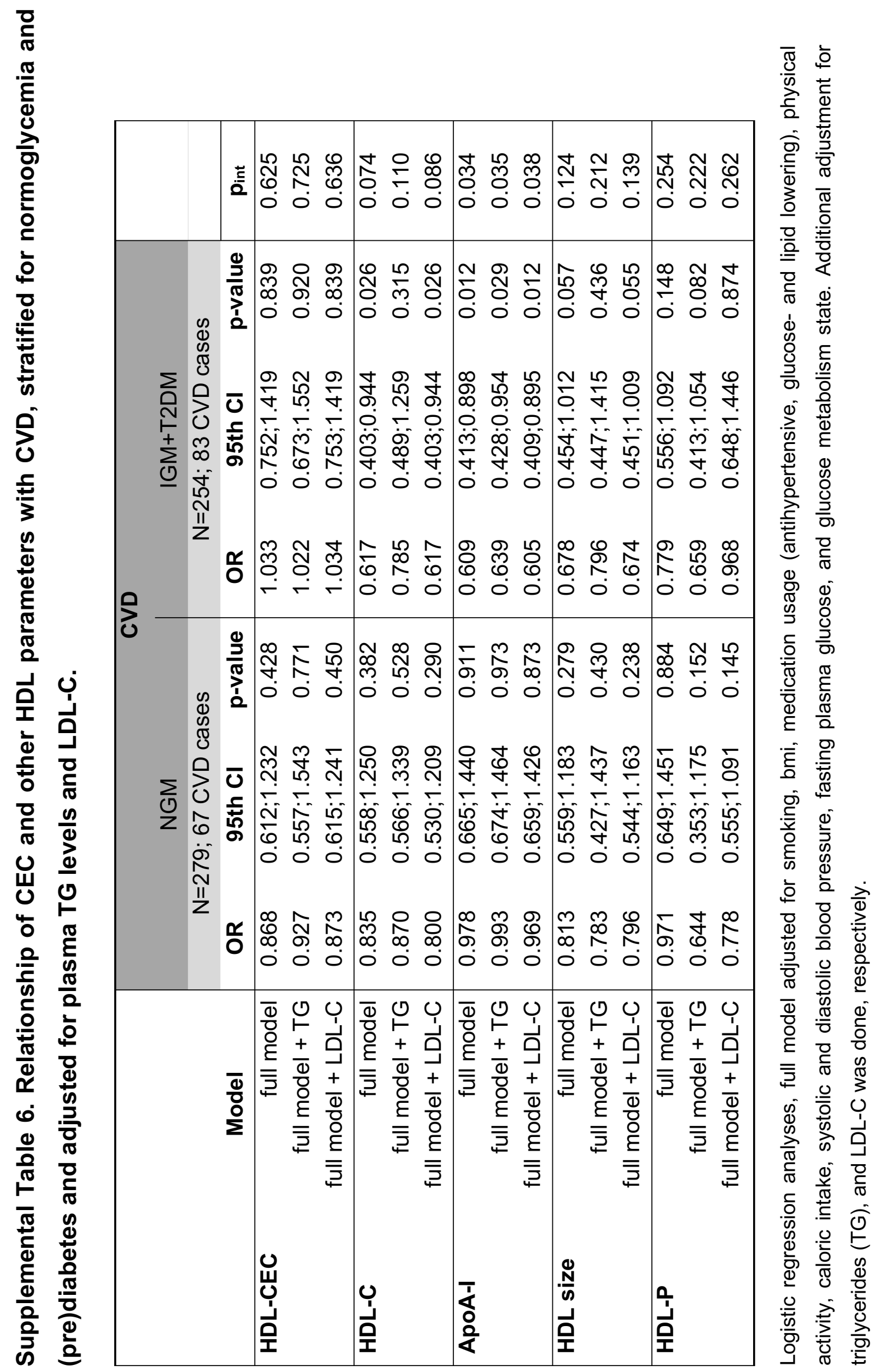


Chapter 2
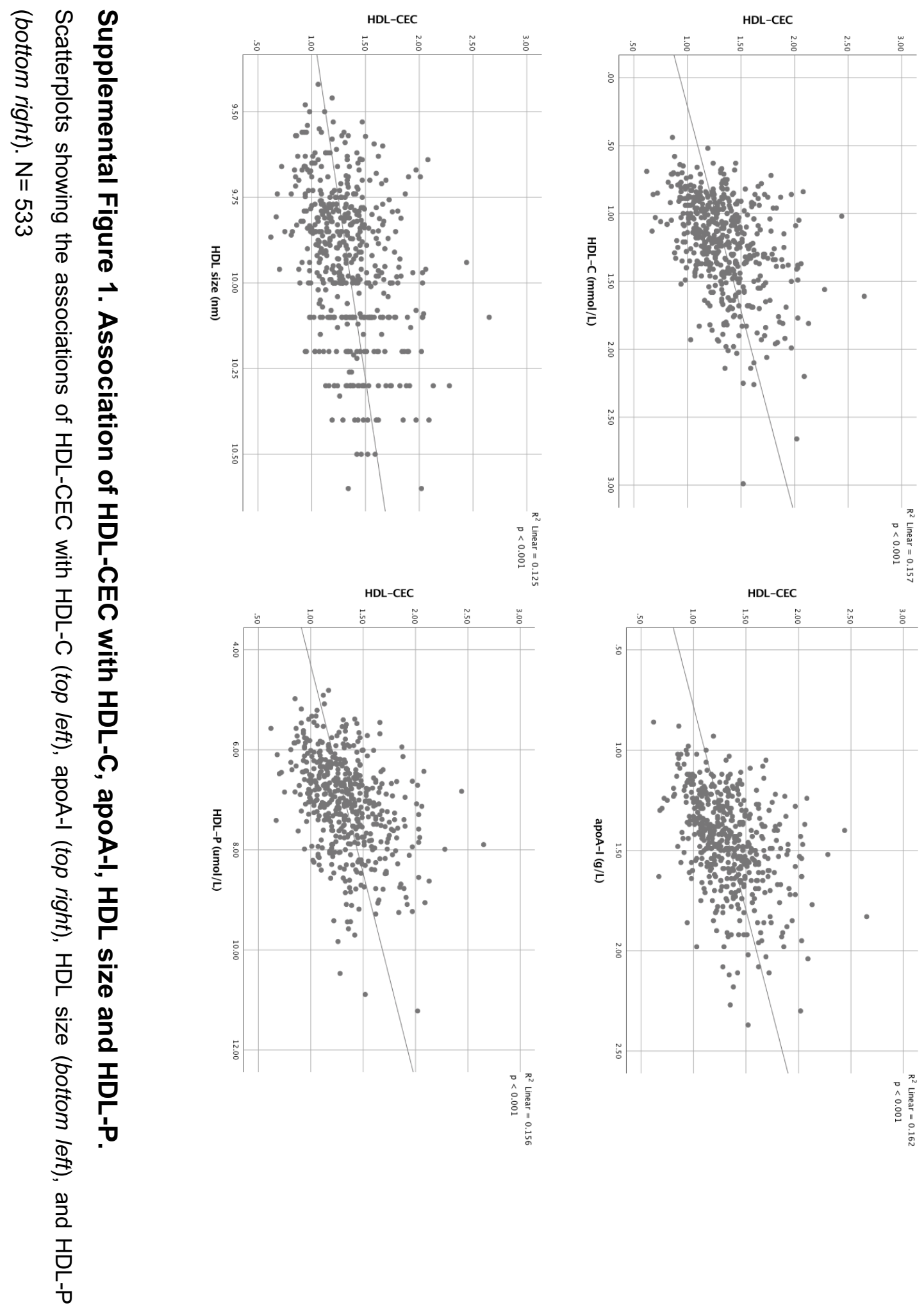


\section{Role of LpL(Lipoprotein Lipase) in Macrophage Polarization In Vitro and In Vivo}

Hye Rim Chang*, Tatjana Josefs*, Diego Scerbo, Namrata Gumaste, Yunying Hu, Lesley-Ann Huggins, Tessa J. Barrett, Stephanie Chiang, Jennifer Grossman, Svetlana Bagdasarov, Edward A. Fisher, Ira J. Goldberg

*equal contribution Arterioscler Thromb Vasc Biol. 2019 Oct;39(10):1967-1985. 


\section{Abstract}

Objective: Fatty acid uptake and oxidation characterize the metabolism of alternatively activated macrophage polarization in vitro, but the in vivo biology is less clear. We assessed the roles of LpL (lipoprotein lipase)-mediated lipid uptake in macrophage polarization in vitro and in several important tissues in vivo.

Approach and Results: We created mice with both global and myeloid-cell specific LpL deficiency. LpL deficiency in the presence of VLDL (very low-density lipoproteins) altered gene expression of bone marrow-derived macrophages and led to reduced lipid uptake but an increase in some anti- and some pro-inflammatory markers. However, LpL deficiency did not alter lipid accumulation or gene expression in circulating monocytes nor did it change the ratio of Ly6Chigh/Ly6Clow. In adipose tissue, less macrophage lipid accumulation was found with global but not myeloidspecific LpL deficiency. Neither deletion affected the expression of inflammatory genes. Global LpL deficiency also reduced the numbers of elicited peritoneal macrophages. Finally, we assessed gene expression in macrophages from atherosclerotic lesions during regression; LpL deficiency did not affect the polarity of plaque macrophages.

Conclusions: The phenotypic changes observed in macrophages upon deletion of $L p /$ in vitro is not mimicked in tissue macrophages.

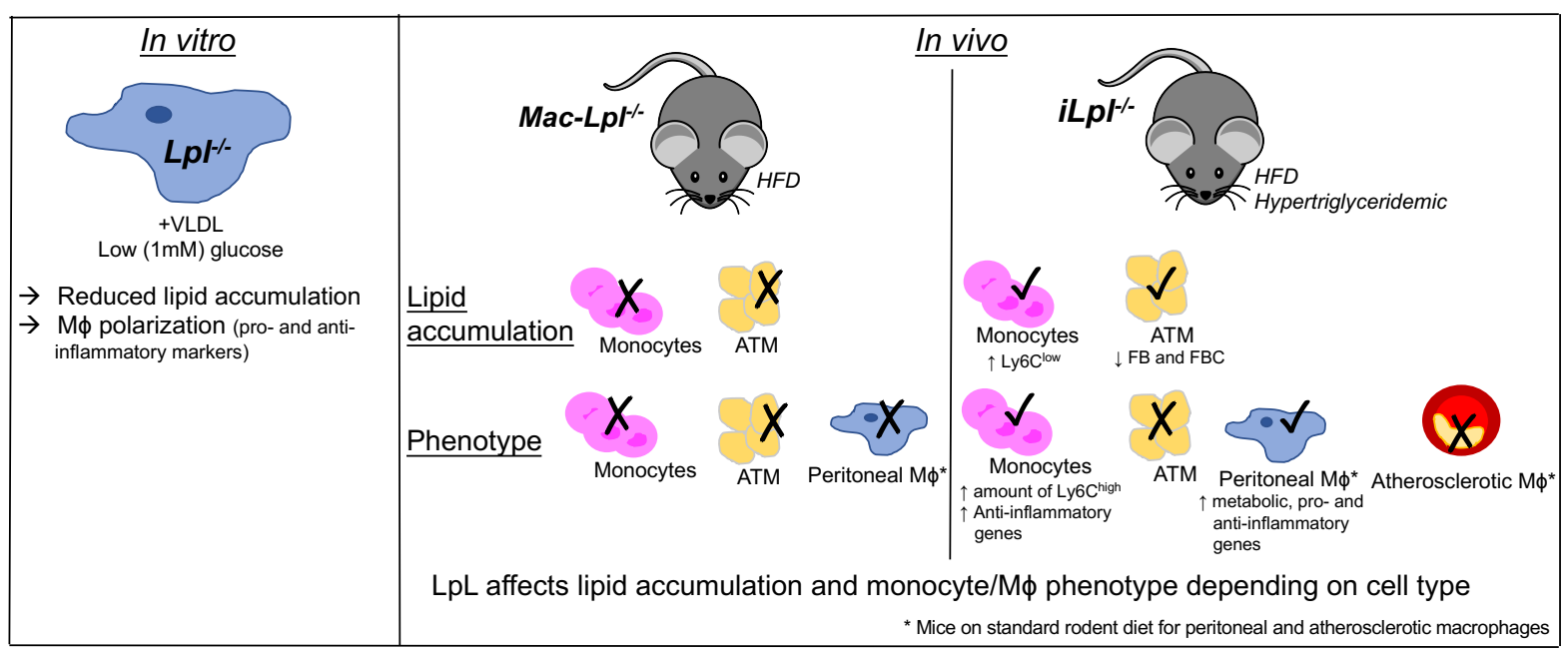




\section{Introduction}

Macrophages (Mфs) are indispensable for maintaining tissue homeostasis and defending against multiple threats as members of the innate immune system. They have essential developmental roles and regulate normal physiology as "first responders" by communicating with the host's adaptive immune system ${ }^{1}$. Although the role and phenotype of different tissue $M \phi s$ are diverse and environment-specific, the $M \phi$ phenotype is often oversimplified as a dichotomy of classically activated $M \phi s$

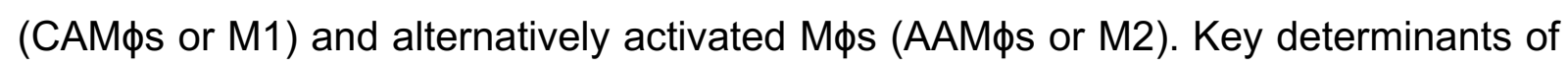
these two phenotypes are thought to include differences in the metabolic pathways used by these cells, and a number of studies, predominantly in vitro, suggest that metabolic substrate use controls $M \phi$ polarization 2,3 .

These two forms of $M \phi s$ defend against different pathogens. CAM $\phi s$ are implicated in acute bacterial infection; they are inflammatory, and their metabolic phenotype is glycolytic and hypoxia inducible factor $1 \alpha$-dependent ${ }^{3}$. AAM $\phi$ s play a significant role in parasitic infection. They utilize fatty acid oxidation (FAO) and play roles in resolving inflammation, including experimental atherosclerosis ${ }^{4}$, and in wound healing. Canonical induction of M2 polarization is mediated by signal transducer and activator of transcription 6 , which transcriptionally upregulates both peroxisome proliferatoractivated receptors (PPARs; PPARס, PPARY) and PPARy coactivator-1 beta (PGC1 $\beta$ ) 3. PPARs and PGC1 $\beta$ activation induce anti-inflammatory markers (Arg1, Ym/Chi3/3, and Fizz1/Relma), FAO-related genes (Cpt1a, Aox $)^{3,5}$, and fatty acid (FA) uptake genes such as cluster of differentiation $36(C d 36)$ and lipoprotein lipase $(L p /)^{5}$.

As noted above, AAM $\phi s$ perform oxidative phosphorylation of $F A s$ and also of glucose ${ }^{6}$ and glutamine ${ }^{7}$, with FAO being the dominant source of energy. Uptake and use of FAs have shown to be critical to conversion of $M \phi s$ to $A A M \phi s$ in vitro ${ }^{5}$, but the in vivo biology is less clear. $L \mathrm{pL}$ is the rate-limiting enzyme for the conversion of circulating triglycerides (TGs), within chylomicrons and very low-density lipoproteins (VLDL), to free FAs (FFAs). It is also responsible, in part, for regulating plasma TG levels. Further, LpL increases the association of lipoproteins with proteoglycans, often referred to as bridging, and hydrolyses circulating lipoprotein, which is critical for lipid uptake in skeletal muscle, heart, and brown adipose tissue (AT) ${ }^{8-10}$. LpL deletion reduces cellular uptake of both FFA and VLDL that occurs via non-receptor (bridging) pathways 11; in vitro studies have documented the importance of this process in monocytes and 
Mфs ${ }^{12-15}$. Notably, macrophage LpL deficiency does not affect receptor-mediated endocytosis $^{16}$. Several in vitro studies have shown that LpL-mediated VLDL lipid accumulation in monocytes induces expression of inflammatory genes such as interleukin-1 beta $(I / 1 \beta)$ and tumor necrosis factor alpha $(\operatorname{Tnf} \alpha){ }^{17,18}$. This indicates that VLDL-derived lipid uptake is inflammatory in monocytes. In contrast, LpL-mediated VLDL lipid uptake has been proposed to suppress $M \phi$ inflammation via PPARס activation ${ }^{19,20}$, suggesting that LpL-mediated lipid uptake promotes an AA phenotype. In contrast to these purported roles in driving M $\phi$ s to a less inflammatory and more reparative phenotype, $M \phi$-derived $L p L$ is atherogenic ${ }^{21}$. These data imply that pathways of lipid uptake and their effects on $M \phi$ polarization found in vitro may differ from what occurs in vivo.

Thus, we asked whether the influences of lipid uptake pathways on M polarity are microenvironment-specific. To answer this, we assessed the roles of $L p L$ in $M \phi$ polarization in vitro and in several important depots in vivo, including AT, the peritoneal cavity, and atherosclerotic plaques. Our data indicate that $L p L$ affects both lipid uptake and $\mathrm{M} \phi$ polarity. The situation in vivo is more complicated as complete LpL deficiency also causes marked hypertriglyceridemia. However, this issue was controlled for, in part, by also studying mice with a myeloid-specific $L \mathrm{pL}$ deletion. Finally, our data show that LpL exerts distinct effects in each tissue depot in vivo, an observation that cannot be easily modeled in vitro.

\section{Materials and Methods}

The authors declare that all supporting data are available within the article and its online supplementary files.

Animals: Global inducible $L p /$ knockout $\left(\mathrm{i} L \mathrm{p}^{-{ }^{-}}\right)$mice were generated by crossing $L p f^{\mathrm{fl} / f l}$ mice with $\mathrm{Tg}^{\text {CreER }}$ transgenic mice (The Jackson Laboratory) ${ }^{22}$, which harbor the tamoxifen-inducible Cre recombinase driven by the chicken beta actin promoter/enhancer coupled with the cytomegalovirus (CMV) immediate-early enhancer ( $\beta$-actin-MerCreMer). Myeloid cell-specific $L p /$ knockout (Mac- $L p{ }^{-{ }^{-}}$) mice ${ }^{21}$ were generated by crossing $L p f^{f l / f l}$ mice with $L y s M C$ re mice (The Jackson Laboratory). Cell culture: Bone marrow (BM) cells were isolated by flushing cells from the femurs or tibiae of mice. Cells were differentiated into BM-derived M $\phi s$ (BMDMs) in normal 
(5 mM) glucose DMEM with $10 \%$ Fetal Bovine Serum (FBS), $1 \%$ penicillin/streptomycin, and murine M-CSF (10 ng/mL; PeproTech) for 7 days. The BMDMs were cultured in low (1 $\mathrm{mM})$ glucose or normal $(5 \mathrm{mM})$ glucose or high (25 $\mathrm{mM}$ ) glucose DMEM for 24 hours with or without murine IL-4 (20 ng/mL; PeproTech) and/or human VLDL (100 $\mathrm{\mu g} / \mathrm{mL}$; Alfar Aesar) plus 5\% FBS for 24 hours.

Plasma Lipid Measurement: Total triglyceride (TG) and total cholesterol (TC) were measured by using Infinity Total Triglyceride Reagent (Thermo Fisher Scientific; \#TR22421) and Infinity Total Cholesterol Reagent (Thermo Fisher Scientific; \#TR13421). Non-esterified FAs (NEFAs) were measured by using Wako Diagnostic NEFA reagents (Wako Life Sciences, Inc).

Lipoprotein Fractionation: Equal amounts of plasma (70 $\mu \mathrm{l}-100 \mu \mathrm{l})$ were used for sequential density ultracentrifugation to separate VLDL $(\mathrm{d}<1.006 \mathrm{~g} / \mathrm{mL})$, low-density lipoprotein (LDL) (d=1.006-1.063 g/mL), high-density lipoprotein (HDL) (d=1.063-1.21 $\mathrm{g} / \mathrm{mL}$ ) in a TLA 100 rotor (Beckmann Instruments). Fractions were used to measure TC and TG as described above.

Glucose and Insulin Measurement: Blood glucose was measured by using OneTouch Ultra2 meter (One Touch). Plasma insulin was measured by using Mouse Ultrasensitive Insulin ELISA kit (Alpco; \#80-ISMSU-E01).

White blood cell counts: Total while blood cell counts in freshly collected mouse blood were obtained using a hematology cell counter (Oxford Science Inc.).

Blood Leukocytes: Monocytes (total and subsets) and neutrophils were identified from whole blood as previously described ${ }^{23}$.

Hematopoietic stem cells: Hematopoietic stem and progenitor cells from the BM and spleen were analyzed by flow cytometry as previously described ${ }^{23}$.

Adipose tissue macrophage (ATM) isolation: ATMs were isolated as previously described ${ }^{24}$.

Peritoneal macrophage isolation: Peritoneal exudate cells were harvested by peritoneal lavage with FACS buffer (PBS+ $0.2 \% B S A+5 \mathrm{mM}$ EDTA) at a volume of 10 $\mathrm{mL}$ per mouse. Total peritoneal exudate cells were sorted by using FACS Ariall (BD Bioscience). The gating strategy to sort large peritoneal M $\phi s$ (LPMs) and small peritoneal M $\phi$ s (SPMs) was as previously described ${ }^{25}$ and is shown in Supplemental Figure 6. 
Seahorse extracellular flux analysis: BMDMs from $L p f^{f / / f l}$ and $i L p l^{-1-}$ were seeded (300,000 cells per well) in $5 \mathrm{mM}$ glucose supplemented with $2 \%$ FBS into XF24 cell culture microplates (Seahorse Bioscience) and stimulated with IL-4 as described above. After 24 hours, oxygen consumption rates (OCR) were measured using an XFe24 instrument (Seahorse Bioscience). For cellular mitochondrial oxidation assessments, the XF Cell Mito Stress Test Kit was used according to the manufacturer's protocol.

Neutral lipid content measurement: Adipo-Red (Lonza) was used according to the manufacturer's protocol to measure intracellular lipid content in BMDMs. Freshly isolated monocytes, ATMs, and peritoneal M $\phi$ s were stained with Bodipy (1:500 dilution; Sigma) for 30 mins in dark on ice to fluorescently label intracellular lipid accumulation. Total neutral lipid content was quantified by using flow cytometry (LSRII) and analyzed by using FlowJo software.

Aortic Transplant. The aortic transplant model has been described before ${ }^{4,26-29} \mathrm{~A}$ plaque burden aortic arch from $L d / r^{\prime-}$ mice was transplanted into the recipient mice (i $L p F^{-1-}$ or $L p f^{f / f l}$ mice), which was inter-positioned with the abdominal aorta. During the transplantation, blood flow was directed through the graft. At time of the transplant, all recipient mice were approximately 22 weeks old and maintained on a normal rodent diet. All mice were sacrificed 14 days after the aortic arch transplantation. AHA guidelines for experimental atherosclerosis studies have been followed ${ }^{30}$.

Laser capture microdissection and quantitative Real Time PCR. CD68+ cells were selected from atherosclerotic plaques by laser capture microdissection. All laser capture microdissection procedures were performed under RNase-free conditions. Aortic root sections were stained with hematoxylin-eosin as previously described ${ }^{31,32}$. Immunohistochemistry and plaque assessment. Grafted arches were removed after perfusion with $10 \%$ sucrose in saline, and embedded in optimum cutting temperature (OCT, Tissue-Tek; Sakura Finetek USA) block and freezed. Serial sections at (6 $\mu \mathrm{m}$ thick) were cut and stained for CD68 (Bio-Rad MCA1957) and CD206, also known as Mannose Receptor (MR) (Bio-Rad MCA2235). Negative controls were performed using an isotype control (Rat IgG2a) instead of the primary antibody (Abcam ab18450). ImagePro Plus 7.0 software was used to determine CD68 ${ }^{+}$ and $\mathrm{MR}^{+}$areas. Overlay was done using Adobe Photoshop CC2018. 
Statistical Analyses: Data are presented as mean \pm SD. Normality test (Shapiro-Wilk normality test $(\alpha=0.05)$ and visual inspection of $Q-Q$ plots) and homogeneity of variance (Brown-Forsythe test) were performed. Depending on the results, parametric or non-parametric tests were chosen as indicated in the figure legends. ${ }^{*} p<0.05$; ${ }^{* *} p<0.01$. All calculations were performed using Microsoft Excel and Graphpad Prism 7.

\section{Results}

LpL deficiency in $M \phi s$ affects their polarization and lipid accumulation in vitro: LpL mediates the hydrolysis of VLDL-derived TG and activates PPARס ${ }^{14}$, which suppresses inflammatory pathways in cultured $M \phi s^{20}$. To assess whether $L p L$ deficiency affects VLDL-dependent lipid uptake and alternative activation (AA) under various culture conditions, BMDMs from wild type and $M \phi$-specific $L p L$ deficient mice (Lp/ffl; LysMCre, Mac-Lpl-/) were cultured with $100 \mu \mathrm{g} / \mathrm{mL}$ of human VLDL plus $5 \%$ FBS for 24 hours in low-glucose (1 mM) containing media. LpL actions enhance $M \phi s$ phagocytic function only in low glucose media ${ }^{16}$ when FAs are likely to be a more critical source of energy substrate. Efficient $L p /$ deletion in BMDMs is shown in Figure 1A. As expected, $L p L$ deficiency led to a marked reduction in FFA in the VLDLcontaining culture system (Figure 1B). Loss of LpL in M $\$$ s significantly reduced intracellular neutral lipids as measured by bodipy and Nile-Red staining (Figure 1C, 1D). To assess whether lack of $L p L$ alters known PPARס-targeted genes, we measured a lipid droplet-related gene, perilipin 2 (Plin2) and a FAO-related gene, carnitine palmitoyltransferase $1 \mathrm{~A}$ (Cpt1a). Consistent with the intracellular lipid droplet results, Plin2 and Cpt1a gene expression were decreased in Mac-Lp ${ }^{-/}$BMDMs (Figure $1 \mathrm{E}, 1 \mathrm{~F})$. In order to examine whether lack of $\mathrm{LpL}$ induces other metabolic-related gene expression as a compensatory mechanism, we measured glucose transporter 1 (Glut1/S/c2a1) and FA synthase (Fasn) mRNA levels. Lpl deficiency increased both Glut1 and Fasn mRNA expression upon VLDL treatment (Figure 1G, 1H), suggesting that lack of LpL-mediated FA uptake increases glucose uptake and de novo FA synthesis. These results demonstrate that $M \phi$-derived $L p L$ is indispensable for VLDLmediated FA uptake under a low glucose culture condition. Next, we investigated whether loss of LpL-mediated VLDL FA uptake impairs M2 polarization and/or augments M1 polarization. Although Arg1 gene expression was decreased, as had been suggested by Chawla et al. ${ }^{5}$, mRNA levels of other M2 markers, Ym1 and Mrc1, 
were increased in Mac- $L p{ }^{-/}$BMDMs in the presence of VLDL, as compared to $L p f^{f / / f l}$ BMDMs (Figure 1I, 1J, 1L). Among inflammatory genes, Tnfa was increased in MacLp ${ }^{-/}$BMDMs (Figure 1M), but Nos2 gene expression showed a trend to decreased expression in Mac-Lp ${ }^{-/}$BMDMs in the presence of VLDL(Figure 10). These results show that lack of $L \mathrm{LL}$ in $\mathrm{M} \phi$ s reduces lipid uptake and alters both inflammatory- and anti-inflammatory genes in VLDL-enriched culture conditions. However, the mixed phenotypic responses indicate a level of complexity in $M \phi$ polarization due to different transcriptional regulation within both the inflammatory and AA pathways.

Figure 1: BMDMs from $L p f^{f / f f l}$ and Mac- $L p{ }^{-/}$mice were used to measure metabolism- related and M1/M2related gene expression in the presence of human VLDL (100ug/mL). (A) The gene expression of $L p l$ in BMDMs. (B) Conditioned media FFAs concentration from cultured BMDMs from $L p{ }^{f / f / l}$ and Mac- $L p F^{--}$ mice. (C) Fluorescent staining of nuclei by DAPI and neutral lipid by bodipy dye in VLDL treated BMDMs (Blue: nuclei, Green: neutral lipids). (D) Quantification of neutral lipid content measured by Nile-red stain in VLDL treated BMDMs (RFU: Relative Fluorescent Unit). (E-F) The expression of PPARס-targeted genes (Plin2, Cpt1a) in BMDMs. (G-H) The expression of Glut1 and Fasn in Mac-Lpr-- BMDMs. (I-L) The gene expression of anti-inflammatory genes (Arg1, Ym1, Fizz1, Mrc1) in VLDL treated BMDMs. (MP) The expression of inflammatory genes (Tnfa, 11b, Nos2, Mcp1) in VLDL treated BMDMs. $\mathrm{N}=6 /$ group. ${ }^{*} p<0.05,{ }^{* *} p<0.01$. Results are represented as median with $25^{\text {th }}$ and $75^{\text {th }}$ percentiles, capped bars indicate $10^{\text {th }}$ and $90^{\text {th }}$ percentile and compared using Two-way ANOVA, Sidak's multiple comparison. 


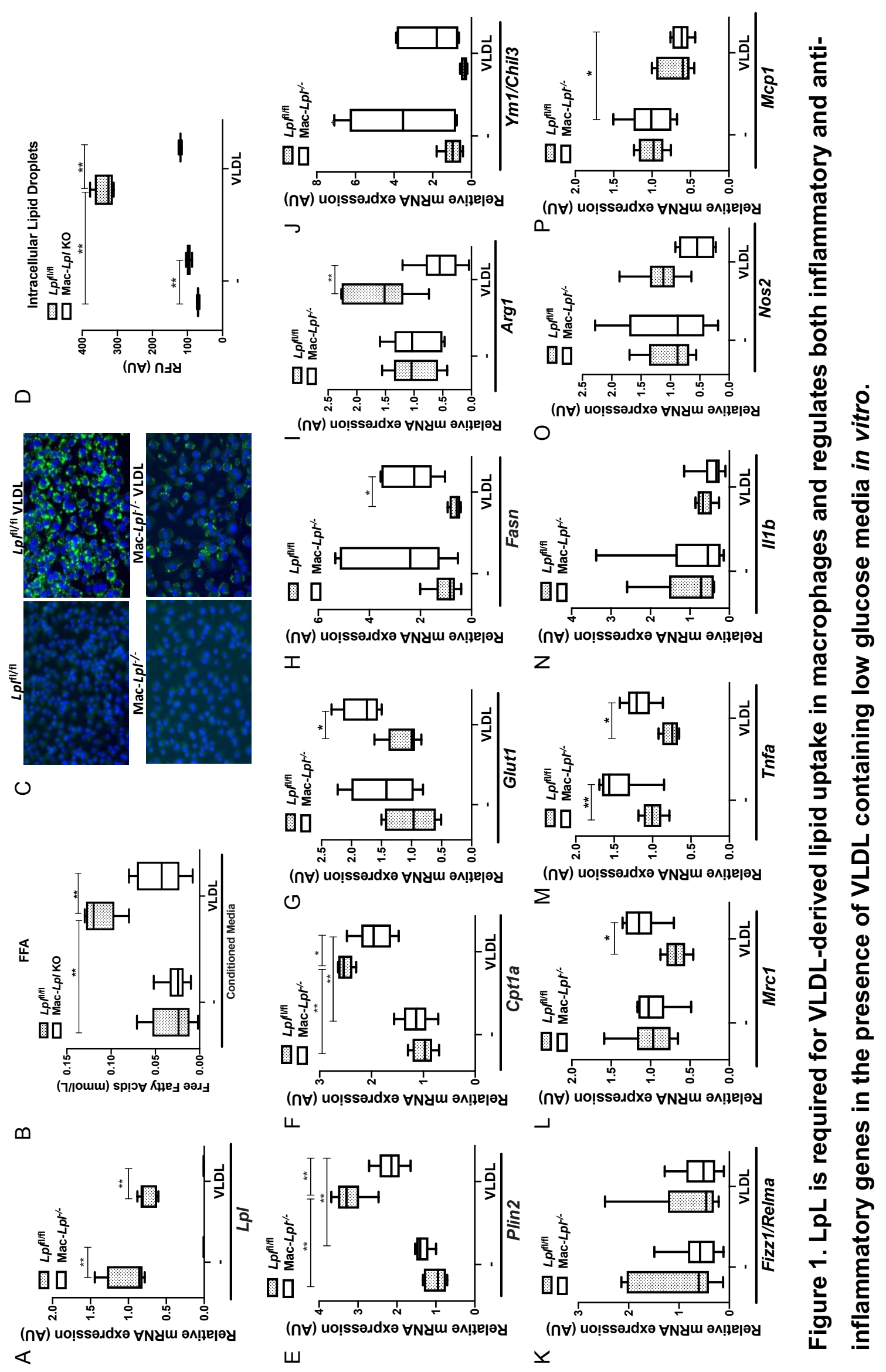


To investigate whether $L p L$ regulates $M \phi$ polarity in response to the standard inducer of AAMs in vitro, we cultured $L p f^{f / f l}$ and Mac- $L p f^{-1-}$ BMDMs with IL-4 $(20 \mathrm{ng} / \mathrm{mL})$ and IL-4 plus VLDL for 24 hours under low glucose conditions. Surprisingly, IL-4 stimuli alone increased Plin2, Cpt1a, and Fasn mRNA levels in Mac-Lpt-1 BMDMs, as compared to $L p^{f / f l \mid}$ BMDMs (Figure 2A, 2B, and 2D). Since IL-4 induces FAO ${ }^{5}$, lack of FA uptake perhaps stimulated cells to increase de novo FA synthesis. Culturing M $\phi s$ with IL-4 plus VLDL led to a significant decrease in Plin2 and Cpt1a, but a trend of increase in Glut1 and a significant 5-fold increase in Fasn gene expression in Mac-Lp ${ }^{-/}$BMDMs (Figure 2A, 2B, 2C, and 2D), similar to in vitro findings in Figure $1 \mathrm{E}-\mathrm{H}$. This suggests that in M2-like M $\phi s$, LpL-mediated VLDL-derived lipid uptake regulates PPARסtargeted genes, but lack of $\mathrm{LL}$ seem to induce metabolic compensations via de novo FA synthesis.

As expected, upon IL-4 treatment alone, lack of LpL prevented up-regulation of Arg1 (Figure 2E). Deletion of LpL did not affect Ym1, Fizz1 and Mrc1 gene expression (Figure 2F-H). Upon IL-4 and VLDL treatment, Lp/ deficiency did not affect Arg1, Ym1, and Mrc1 but dramatically increased Fizz1/Relma expression. Huang et al. demonstrated that glucose-mediated FA synthesis is necessary for M2 activation as measured by Fizz1/Relma and programmed death-ligand 1 expression ${ }^{6}$. Thus, upregulation of Fizz1 expression is probably due to glucose-mediated FA synthesis, as we found an increase in both Glut1 and Fasn mRNA levels in Mac-Lp ${ }^{-1}$ BMDMs. To test the metabolic status of $M \phi s$ in $\mathrm{LpL}$ depleted conditions, we performed OCR experiments in cultured $L p^{f / f / f}$ and Mac- $L p F^{-1-}$ BMDMs incubated with IL-4 $(20 \mathrm{ng} / \mathrm{mL})$ (Figure $2 \mathrm{I}-\mathrm{J}$ ). The results showed that OCR is increased when $L \mathrm{LL}$ is depleted, being in accord with the changes we found in Cpt1a gene expression. Overall, our data show that in $A A M \phi s, L p L$ is necessary for FA uptake, but it is not anti-inflammatory under the low glucose condition in vitro. Further, increase in Glut1 mRNA level suggests that $\mathrm{LpL}$ deficient M $\phi$ s switch to a greater use of glucose. 

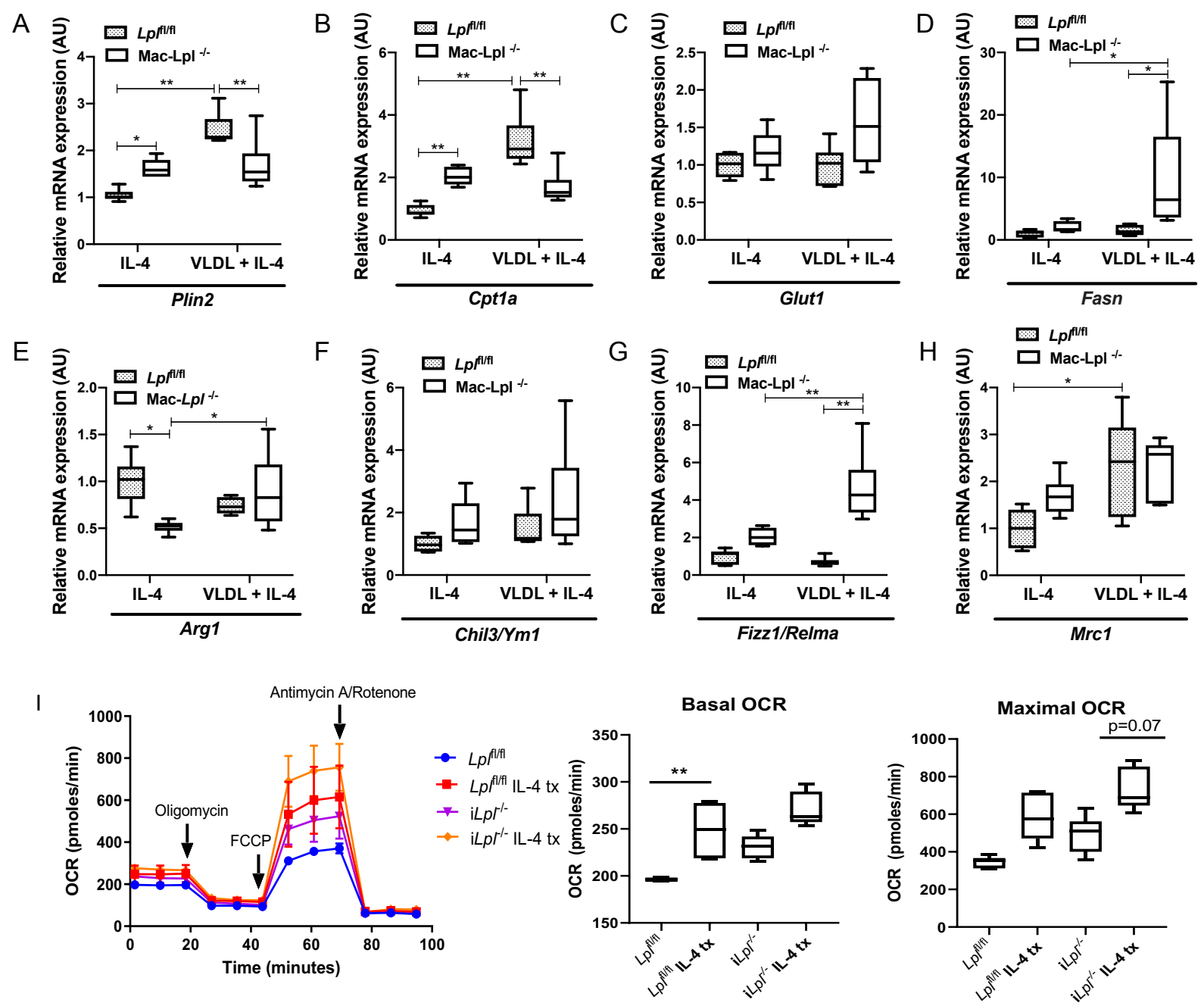

Figure 2. LpL is necessary for VLDL-derived FA uptake, but it is not antiinflammatory under low-glucose condition in vitro. BMDMs from $L p f^{f / f l}$ and Mac- $L p F^{-1-}$ mice were used to measure metabolism-related and M2-related genes in the presence of IL-4 $(20 \mathrm{ng} / \mathrm{mL})$ or IL-4 plus human VLDL. (A) The expression of a lipid droplet-related protein, Plin2, gene in BMDMs. (B) The expression of a fatty acid oxidation-related gene, Cpt1a in BMDMs. (C) The expression of a glucose transporter, Glut1 (SIc2a1), gene in BMDMs. (D)The expression of fatty acid synthase gene in BMDMs. (E-H) The expression of canonical M2 (anti-inflammatory) genes in BMDMs. (I) Oxygen consumption rate comparing IL-4 treatment in $L p f^{f / / f l}$ and Mac- $L p f^{{ }^{-}} . \mathrm{N}=3 /$ group. ${ }^{*} \mathrm{p}<0.05,{ }^{* *} \mathrm{p}<0.01$. Results are represented as median with $25^{\text {th }}$ and $75^{\text {th }}$ percentiles, capped bars indicate $10^{\text {th }}$ and $90^{\text {th }}$ percentile and compared compared using Two-way ANOVA with Sidak's multiple comparison test.

To test whether FFAs not derived from LpL-hydrolysis alter the phenotype of Mac- $L \mathrm{pl}^{-/-}$ BMDMs, we incubated BMDMs with or without oleic acid (OA) (300 $\mu \mathrm{M})$ for 24 hours. OA significantly decreased $L p /$ expression, but increased Cpt1a gene expression in $L p f^{f / / f l}$ BMDMs (Supplemental Figure 1). OA treatment also significantly lowered Mrc1 
gene expression in Mac- $L p{ }^{{ }^{--}}$BMDMs with a trend towards decreased expression in $L p f^{f / / f l}$ BMDMs. Unexpectedly, we did not find altered expression of other antiinflammatory related-genes such as Ym1/Chil3 or Fizz1/Relma, whereas inflammatory genes increased upon OA treatment (Supplemental Figure 1). Although OA treatment is generally known to induce M2 genes (Arg1, Mrc1) ${ }^{33-35}$, our data suggest that OAdependent M2 activation can be time- or dose-dependent. The pattern of gene expression in response to OA treatment was similar in $L p f^{f / f l}$ compared to Mac- $L \mathrm{pl}^{-/-}$ BMDMs, suggesting that FA uptake does regulate anti-inflammatory or inflammatory gene expression independent of $L \mathrm{pL}$ expression, whereas VLDL-derived FA requires LpL expression to invoke an inflammatory response.

In order to determine whether increasing glucose concentration from low glucose culture conditions ( $1 \mathrm{mM})$ to normal $(5 \mathrm{mM})$ affects $\mathrm{M} \phi$ polarity in vitro, we performed the same experiment with both media. In the normal glucose condition, $L p /$ deletion increased Plin2, Cpt1a, and Fasn mRNA levels (Supplemental Figure 2A, 2B and 2E) but did not alter Glut1 expression in the presence of VLDL (Supplemental Figure 2D). These data indicate that under the normal glucose condition, in vitro, M $\phi$ s mainly utilize glucose as the energy fuel as they do in vivo ${ }^{36}$; thus, lack of LpL-mediated FA uptake does not impact Glut1 expression. With regards to $M \phi$ polarity, similar to low glucose culture conditions, Arg1 was significantly decreased in Mac- $L \mathrm{pl}^{-/-}$BMDMs, whereas other typical M2 genes such as $Y m 1$ and Fizz1 were up-regulated in the presence of VLDL (Supplemental Figure 2F, 2G, and 2H). Upon IL-4 stimulation in the normal glucose condition, we found a similar pattern to the low glucose conditions, suggesting that increasing glucose concentration from $1 \mathrm{mM}$ to $5 \mathrm{mM}$ does not significantly impact $L p /$ deficient $M \phi$ polarity. The changes that we observe only matter to M2-induced conditions using VLDL and/or IL-4, as culture conditions without substrate added to the enzyme and no induction for anti-inflammation served as baseline conditions.

Since our results show that $L p l$ deficiency up-regulates Glut1 and Fasn gene expression under low glucose and up-regulates Fasn gene expression under the normal glucose culture condition, we hypothesized that glucose-mediated FA synthesis is responsible for increasing some M2-related genes ( $Y m 1$ and Fizz1). Thus, we performed the same in vitro experiment under high $(25 \mathrm{mM})$ glucose conditions to examine whether an excess amount of glucose impacts both metabolic- and M2related genes. Unlike low and normal glucose culture conditions, we found no 
significant difference between $L p /$ expressing and non-expressing cells in all M2related genes (Arg1, Ym1, Fizz1, Mrc1) in high glucose containing media (Supplemental Figure $3 \mathrm{G}, 3 \mathrm{H}, 3 \mathrm{l}$ and $3 \mathrm{~J}$ ). These data indicate that with excess glucose, M $\phi$ s mainly use glucose as their energy source; thus, ablating LpL-mediated FA uptake does not affect either metabolism or polarization.

\section{The impact of myeloid-specific $L p /$ ablation on circulating monocytes in obese}

mice: While we could reproduce many of the in vitro findings of previously reported studies ${ }^{14}, 19$, we found that these effects varied by culture condition. For this reason, we next investigated the role of $L p L$ in monocytes and $M \phi s$ in a lipid-rich microenvironment in vivo. In order to induce a hyperlipidemic condition, we used mice fed a HFD. Obesity increases circulating levels of myeloid-derived cells ${ }^{37}$. This diet also enables us to examine ATMs, which are limited in animals fed a normal rodent diet. Thus, we sought to determine whether myeloid cell-derived $\mathrm{LpL}$ regulates monocyte subpopulations (Ly6 $\mathrm{C}^{\text {hi }}$ and Ly6Clow monocytes), lipid accumulation, and phenotype of monocytes. The detailed experimental design is shown in Figure 3A. We confirmed that $L p /$ was efficiently deleted in monocytes in Mac- $L p H^{-1}$ mice (Figure $3 \mathrm{~B}$ ). Ablation of $L p /$ in myeloid-derived cells with HFD did not affect plasma glucose levels (Figure $3 \mathrm{C}$ ). As expected, levels of plasma TG and TC in VLDL, LDL, and HDL in Mac- $L p^{H^{-1}}$ mice were not significantly different from TG and TC levels in $L p f^{f / f f l}$ mice (Figure 3D, 3E). Lack of $L p L$ in monocytes with HFD did

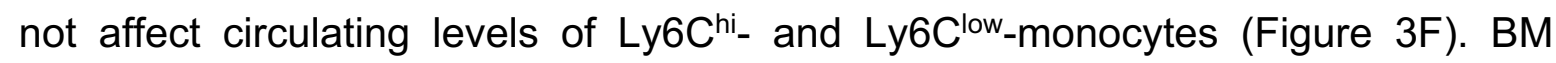
progenitors (common myeloid progenitors and granulocyte-M $\phi$ progenitors) in Mac$L p H^{-1-}$ mice were not significantly different from $L p^{f / / f l}$ mice (Supplemental Figure $4 A$ ), as circulating levels of monocytes were unaltered. Thus, differences in BM progenitors and circulating monocytes noted with normal rodent diets ${ }^{38}$ were abrogated by the HFD, which is known to stimulate BM progenitors ${ }^{37}$. Ablation of $L p l$ did not alter intracellular lipid content as measured by percent of bodipy+cells from each monocyte population (Figure 3G, 3H) and bodipy+ geometric mean fluorescent intensity (gMFI) (Figure 3l). Consistent with the intracellular lipid content, expression of Plin2 and other lipid-related genes were unaltered in Mac-L $\mathrm{F}^{-1-}$ mice (Figure $3 \mathrm{~J}$ ). Also, $L p /$ deficiency in monocytes did not lead to an increase in inflammatory gene expression (Figure 3K) and did not affect expression of anti-inflammatory-related 
genes (Figure $3 \mathrm{~L}$ ). These results indicate that myeloid cell-derived $\mathrm{LpL}$ in vivo is not required in monocytes for lipid accumulation and is not inflammatory in these cells.

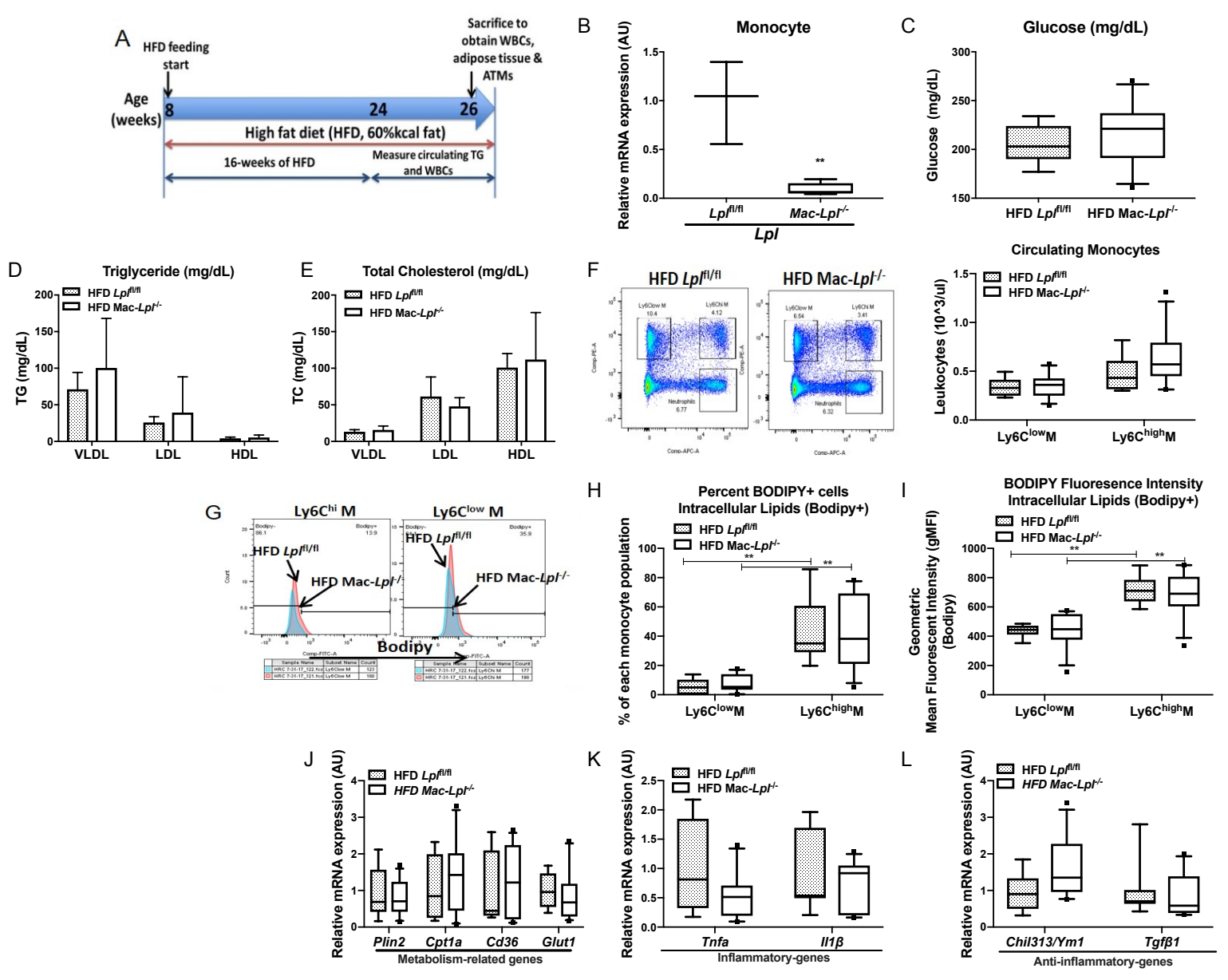

Figure 3. Myeloid-cell derived $\mathrm{LpL}$ does not affect systemic metabolism and circulating levels of monocytes, and is not required for lipid accumulation in

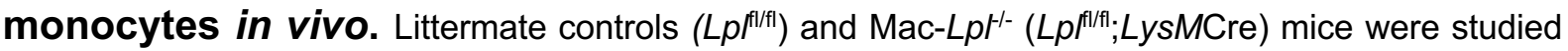
for plasma metabolic parameters, circulating levels of monocytes, and lipid content and phenotype of monocytes. (A) Experimental design: $L p f^{f / f f l}$ and Mac- $L p{ }^{-/}$mice were fed with a HFD (60\%kcal fat) from 8 weeks old of age until sacrifice (26 weeks old of age); Body weight and plasma parameters were measured between 24 weeks of age and 25 weeks of age; Circulating monocytes (Ly6C $C^{\text {hi }}$ and Ly6 $\mathrm{C}^{\text {low }}$ ), adipose tissue (PGAT, SCAT, and BAT), and adipose tissue macrophages (CD45 ${ }^{+} \mathrm{F} 4 / 80^{+} \mathrm{CD} 11 \mathrm{~b}^{+}$and $\mathrm{CD} 45^{+} \mathrm{F} 4 / 80^{+} \mathrm{CD} 11 \mathrm{~b}^{+} \mathrm{CD} 11 \mathrm{c}^{+}$) were obtained at 26 weeks old of age. (B) The expression of $L p /$ in monocytes $\left(C D 45^{+} C D 115^{+}\right)$that were isolated by fluorescence-activated cell sorting (FACS) method. (C) Plasma fasting glucose level. (D) Levels of plasma triglyceride in VLDL, LDL, and HDL fraction. (E) Levels of plasma total cholesterol in VLDL, LDL, and HDL fraction. (F) Representative flow cytometry plots of blood leukocytes and quantified number of circulating Ly6 $\mathrm{C}^{\text {hi }}$ and Ly6Clow monocyte. (G)

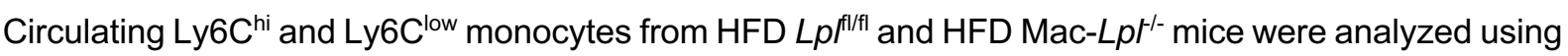


flow cytometry for neutral lipid content (BODIPY); Representative flow cytometry histogram plots are shown for BODIPY fluorescence. (H) Quantified percentage of BODIPY fluorescence in Ly6C $\mathrm{C}^{\text {hi }}$ and Ly6C ${ }^{\text {low }}$ monocytes. (I) Quantification of BODIPY geometric mean fluorescence intensity (gMFI) in Ly6C ${ }^{\text {hi }}$ and Ly6Clow monocytes. $(\mathrm{J})$ The expression of metabolism-related genes (Plin2, Cpt1a, Cd36, Glut1) in total monocytes. (K) The expression of inflammatory genes (Tnfa, II1b) in total monocytes. (L) The expression of anti-inflammatory genes $(Y m 1$ and $T g f b i)$ in total monocytes. $N=6 /$ group. ${ }^{*} \mathrm{p}<0.05$, ${ }^{* *} p<0.01$. Results are represented as mean $\pm S D(B, D, E)$ and median with $25^{\text {th }}$ and $75^{\text {th }}$ percentiles, capped bars indicate $10^{\text {th }}$ and $90^{\text {th }}$ percentile $(F-L)$ and $L p f^{f / / f l}$ and Mac- $L p H^{--}$compared using unpaired $t$-test (B-E, J-L). Two-way ANOVA with Sidak's multiple comparison test was used for (F-I).

The effect of $L p /$ deletion on ATMs: Next, we investigated whether deletion of $L p L$ in myeloid-derived cells affects ATM lipid uptake and polarity. Visceral AT-specific acute silencing of $L p /$ in ATMs using glucan-encapsulated siRNA particles showed that $L p l$ deficiency in ATMs slightly decreased ATM neutral lipid content in leptin deficient (ob/ob) mice ${ }^{39}$. However, it is less clear whether LpL affects subpopulations and polarization. Because acute $L p /$ silencing affected lipid content in ATMs in vivo ${ }^{37}$ and we found a significant reduction in lipid content of BMDMs in vitro (Figure 1), we postulated that $L \mathrm{pL}$ affects the phenotype of $M \phi s$ residing in a high-fat environment. For that reason, we first investigated the role of $L \mathrm{pL}$ in ATMs of obese mice.

ATMs are thought to consist of two major subpopulations: F4/80+CD11b (FBs) and $\mathrm{F} 4 / 80+\mathrm{CD} 11 \mathrm{~b}+\mathrm{CD} 11 \mathrm{c}+(\mathrm{FBCs})$. Although several studies described that $\mathrm{FBs}$ are less inflammatory, while FBCs are more inflammatory ${ }^{40}$, Xu et al. ${ }^{24}$ demonstrated that FBCs have more neutral lipid content, increased transcriptional profile of lysosomaldependent lipid metabolism, and are not more inflammatory than FBs. So, the data are inconsistent. In our experiments, ablation of $\mathrm{LpL}$ in myeloid-derived cells did not alter total ATM $(\mathrm{F} 4 / 80+)$ content (Figure $4 \mathrm{~A})$. Unexpectedly, we found that $L p /$ deficiency in ATMs did not lead to fewer Cd11C+ ATMs (Figure 4B, 4C) or less lipid accumulation measured by percent of bodipy+ cells (Figure 4D, 4E) and bodipy+ intensity as measured by bodipy gMFI (Figure $4 \mathrm{E}$ ). Upon $L p /$ deletion, $L p /$ deficient ATMs showed approximately $20 \%$ higher Glut1 mRNA expression than control M $\phi$ s (Figure $4 G$ ), suggesting a slight metabolic shift from FA uptake to glucose uptake in ATMs. Inflammatory and anti-inflammatory gene expression did not change (Figure $4 \mathrm{H}, 4 \mathrm{I}$ ). Our findings are similar to a previous report showing that myeloid cell-specific GLUT1 overexpression did not alter inflammatory phenotype in vivo ${ }^{36}$. Here, we illustrate that 
LpL is not necessary for the development of more lipid-laden ATMs (FBCs), intracellular lipid accumulation, and ATM polarization.

These results would not be expected if LpL-mediated lipolysis was needed to supply lipids and to activate PPARs, as occurs in other cells and tissues, such as the heart and skeletal muscle ${ }^{41}$. Therefore, lipid supply to circulating monocytes and $M \phi s$ must either come from FFAs, uptake of remnant lipoproteins that were partially hydrolyzed by LpL in other tissues, or endocytosis of TG-rich lipoproteins followed by intracellular lipolysis or de novo lipogenesis.
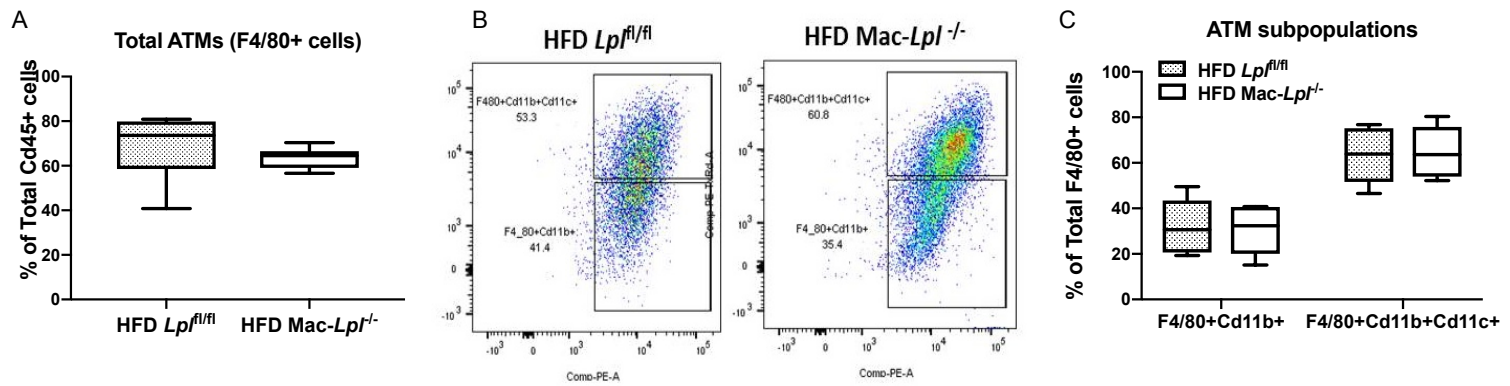

D

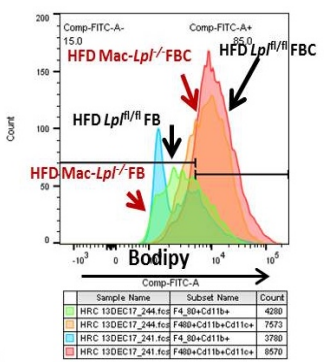

E

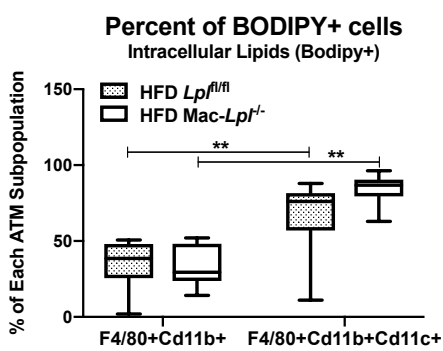

$\mathrm{H}$
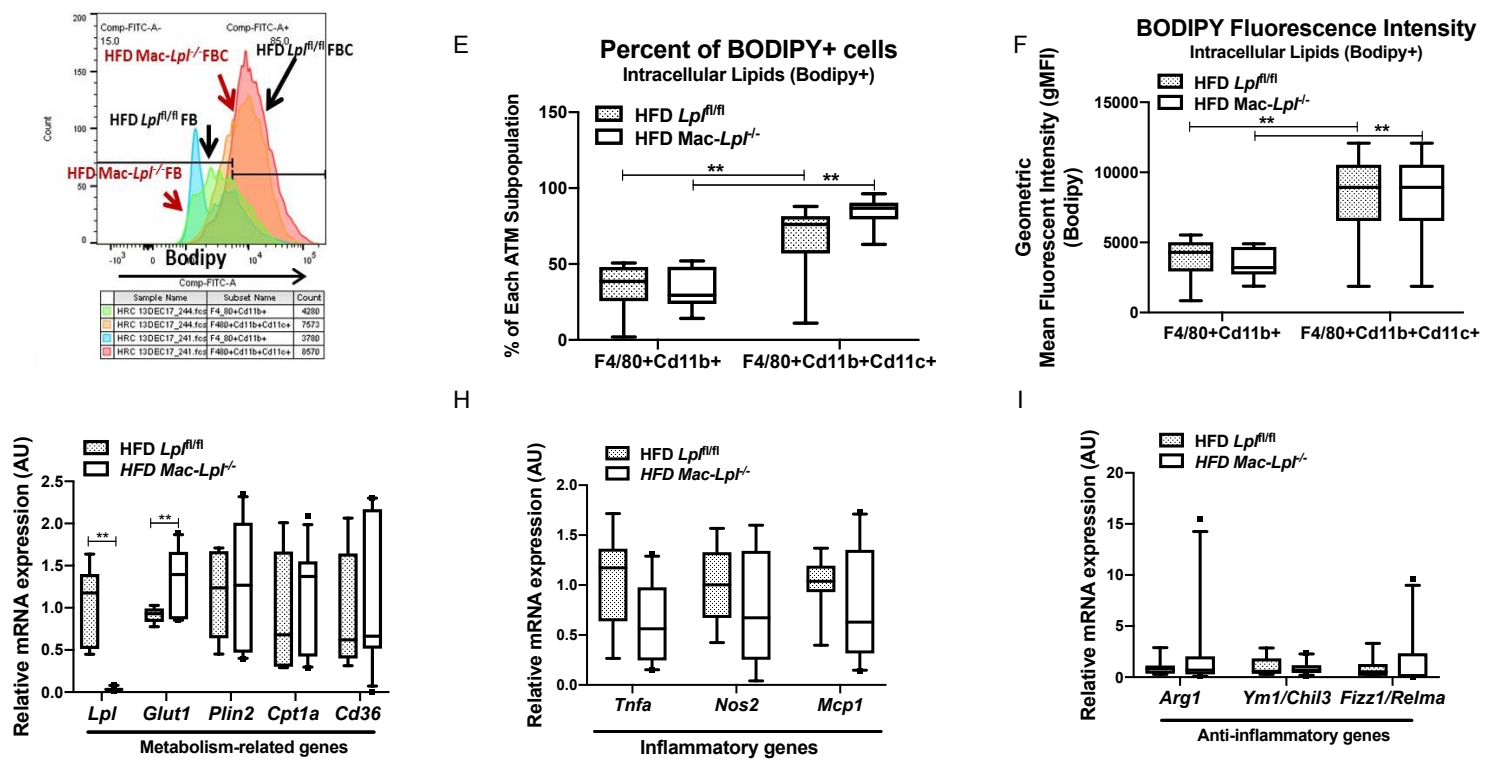

Figure 4. Myeloid-cell derived $L p L$ is not required for more lipid-laden ATM development (FBCs), intracellular lipid accumulation, and ATM polarization.

HFD fed littermate controls $\left(L p^{f / f f l}\right)$ and Mac- $L p{ }^{H^{-}}\left(L p^{f / f f l} ; L y s M C r e\right)$ mice were studied for total ATM $(\mathrm{CD} 45+\mathrm{F} 4 / 80+) \quad$ content, $\mathrm{ATM}$ subpopulation $(\mathrm{CD} 45+\mathrm{F} 4 / 80+\mathrm{Cd} 11 \mathrm{~b}+$ and $\mathrm{CD} 45+\mathrm{F} 4 / 80+\mathrm{CD} 11 \mathrm{~b}+\mathrm{CD} 11 \mathrm{c}+$ ), and ATM polarity. (A) Flow cytometry analysis of total ATMs (CD45+F4/80+). (B) Representative flow cytometry plots for ATM subpopulations; FBs $(\mathrm{CD} 45+\mathrm{F} 4 / 80+\mathrm{Cd} 11 \mathrm{~b}+)$ and FBCs $(\mathrm{CD} 45+\mathrm{F} 4 / 80+\mathrm{CD} 11 \mathrm{~b}+\mathrm{CD} 11 \mathrm{c}+)$ and quantified FBs and FBCs. (C) Quantified percentage of FBs and FBCs from CD45+F4/80+ cells. (D) Representative flow cytometry histogram plots are shown for BODIPY fluorescence in FBs and FBCs from HFD $L p f^{f / f f l}$ and HFD 
Mac- Lp ${ }^{\text {- }}$ mice. (E) Quantified percentage of BODIPY fluorescence in FBs and FBCs. (F) Quantification of BODIPY geometric mean fluorescence intensity (gMFI) in FBs and FBCs. (G) The expression of metabolism-related genes ( $L p l$, Glut1, Plin2, Cpt1a, Cd36) in FBCs. $(\mathrm{H})$ The expression of inflammatory genes (Tnfa, Nos2, Mcp1) in FBCs. (I) The expression of anti-inflammatory genes (Arg1, Ym1, Fizz1) in total FBCs. $N=6$ /group. ${ }^{*} p<0.05,{ }^{* *} p<0.01$. Results are represented as median with $25^{\text {th }}$ and $75^{\text {th }}$ percentiles, capped bars at $10^{\text {th }}$ and $90^{\text {th }}$ percentile and compared using Two-Way ANOVA, Sidak's multiple comparison test (A-F) or unpaired t-test, or Mann-Whitney Test (Glut1 Plin2, Cd36, Arg1, Ym1/Chil3, Fizz1/Relma) (G-I).

The impact of inducible total body $L p /$ deletion in myeloid-derived cells in obesity: To determine whether deletion of $\mathrm{LpL}$ in myeloid-derived cells is compensated by LpL in other tissues, we analyzed monocytes and ATMs in $i L \mathrm{p}^{-/}$and

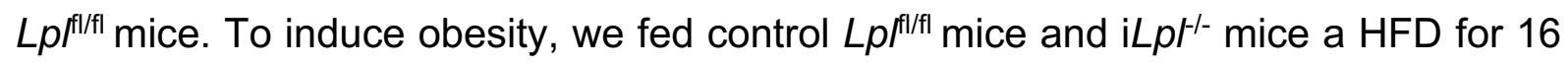
weeks. Tamoxifen was injected intraperitoneally into both groups, globally ablating $L p l$ in the experimental (i $L \mathrm{pl}^{-/}$) group (Figure $5 \mathrm{~A}$ ) ${ }^{22,42}$. We confirmed efficient $L p /$ deletion by measuring $L p /$ expression in AT (Figure $5 \mathrm{~B}$ ).

The circulating glucose level was decreased in HFD iL $\mathrm{F}^{-1-}$ mice (Figure $5 \mathrm{C}$ ), suggesting that lack of LpL-mediated lipid uptake causes an increase in glucose uptake into tissues as was found with neonatal $L p L$ deficiency ${ }^{43}$. As expected, global $L p /$ deletion also increased VLDL-derived TG levels from $88 \mathrm{mg} / \mathrm{dL}$ to $4000 \mathrm{mg} / \mathrm{mL}$ as well as LDLand HDL-TG (Figure 5D). VLDL-derived TC levels significantly increased, whereas LDL-TC and HDL-TC decreased in iLp ${ }^{-1-}$ mice (Figure 5E).

Induced loss of $L p L$ also altered circulating white bloods cells in these HFD-fed mice. Ly6 $\mathrm{C}^{\text {hi }}$ circulating monocytes were significantly increased in HFD iLpL $L^{--}$mice relative to HFD $L p f^{f / f / f l}$ mice (Figure 5F). The increase in levels of circulating Ly6 $\mathrm{C}^{\text {hi }}$ monocytes occurred with increased granulocyte-M $\mathrm{\phi}$ progenitors in bone marrow (Supplemental Figure 4D). Although percentage of bodipy+ cells in both Ly6Clow and Ly6Chigh monocytes was unaltered in HFD i $\mathrm{pr}^{-1-}$ mice (Figure 5G, 5H), we found that bodipy+ gMFI was significantly higher in Ly6C $\mathrm{C}^{\text {low }}$ monocytes as compared to those in $L p f^{\text {fl/fl }}$ mice (Figure 5I). These intracellular lipid content results indicate that although monocytes from both HFD $L p f^{f / f l}$ and $i L p L^{-1-}$ mice are fully loaded with lipid droplets, lipid droplet size and number are greater in monocytes in HFD i $L \mathrm{pr}^{-1-}$ mice. Thus, these lipids are obtained via a non-LpL-mediated lipid uptake. 
To study the transcriptional profile and determine the role of $L p L$ in monocytes in HFD $\mathrm{i} \mathrm{Lp}^{-1-}$ mice, we used RNA sequencing as an unbiased approach. As described previously, we analyzed inflammatory- and anti-inflammatory genes, as well as metabolism-related genes. An inflammatory gene, Tnfa, was significantly decreased, but $I / 1 \beta$ was unaltered in HFD iLp ${ }^{--}$mice (Figure $5 \mathrm{~K}$ ), while anti-inflammatory genes were up-regulated (Tgfbi, Ym1/Chi313) in HFD iLp/-- mice (Figure 5L). CD36 mRNA levels were significantly downregulated in HFD $\mathrm{i} L \mathrm{p}^{-/}$- mice, whereas other metabolismrelated genes showed a trend towards increased expression (Plin2, Cpt1a) or were unaffected (Figure 5J). However, KEGG pathway analysis showed that lack of $L p l$ increased oxidative phosphorylation and lysosome-related pathways (Supplemental Figure 7), suggesting that $L p /$ deficiency leads to a metabolic reprogramming by increasing endocytic TG-rich uptake for more oxidative phosphorylation. These data show that hypertriglyceridemia caused by global $L p /$ deletion with HFD induces BM progenitors to produce more Ly6 $\mathrm{C}^{\text {hi }}$ monocytes. Also, hypertriglyceridemia in HFD $\mathrm{i} L \mathrm{pF}^{\text {- }}$ mice leads to an increase of lipid content in Ly6 $\mathrm{C}^{\text {low }}$ monocytes, which induces M2 gene expression but decreases a M1 gene, Tnfa, in monocytes.

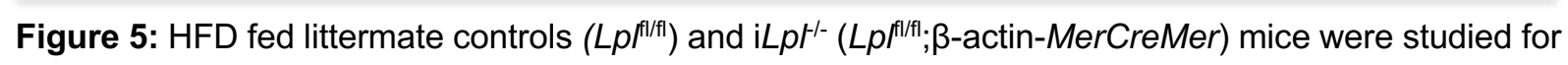
plasma metabolic parameters, circulating levels of monocytes, and lipid content and phenotype of monocytes. (A) Experimental design: $L p f^{\text {flffl }}$ and $i L p{ }^{-1}$ mice were fed with a HFD from 8 weeks old of age for until sacrifice (30 weeks old of age); Body weight and plasma parameters were measured between 24 weeks of age and 30 weeks of age; Circulating monocytes (Ly6C $C^{\text {hi }}$ and Ly6C ${ }^{\text {low }}$ ), adipose tissue (PGAT, SCAT, and BAT), and adipose tissue macrophages $\left(C D 45^{+} \mathrm{F} 4 / 80^{+} \mathrm{CD} 11 \mathrm{~b}^{+}\right.$and $\left.\mathrm{CD} 45^{+} \mathrm{F} 4 / 80^{+} \mathrm{CD} 11 \mathrm{~b}^{+} \mathrm{CD} 11 \mathrm{c}^{+}\right)$were obtained at 30 weeks old of age. (B) The expression of $\mathrm{Lpl}$ in adipose tissue ( $N=4-5 / g r o u p)$. (C) Plasma fasting glucose level ( $N=11-17 / g r o u p)$. (D) Levels of plasma triglyceride in VLDL, LDL, and HDL fraction (N=4-5/group). (E) Levels of plasma total cholesterol in VLDL, LDL, and HDL fraction (N=4-5/group). ( $F$ ) Representative flow cytometry plots of blood leukocytes and quantified number of circulating Ly6 $\mathrm{C}^{\text {hi }}$ and Ly6 $\mathrm{C}^{\text {low }}$ monocyte (N=11-17/group). (G) Circulating Ly6 $\mathrm{C}^{\mathrm{hi}}$ and $L y 6 \mathrm{C}^{\text {low }}$ monocytes from HFD $L p f^{\text {fl/fl }}$ and HFD i $L p{ }^{{ }^{-1}}$ mice were analyzed using flow cytometry for neutral lipid content (BODIPY); Representative flow cytometry histogram plots are shown for BODIPY fluorescence ( $\mathrm{N}=3-5 /$ group). ( $\mathrm{H}$ ) Quantified percentage of BODIPY fluorescence in Ly6 $\mathrm{C}^{\text {hi }}$ and Ly6 $\mathrm{C}^{\text {low }}$ monocytes ( $\mathrm{N}=3-5 /$ group). (I) Quantification of BODIPY geometric mean fluorescence intensity (gMFI) in Ly6C $\mathrm{C}^{\text {hi }}$ and Ly6C $\mathrm{C}^{\text {low }}$ monocytes (N=3-5/group). ( $\mathrm{J}$ ) The expression of metabolism-related genes (Plin2, Cpt1a, Cd36, Glut1) in total monocytes (N=4/group). (K) The expression of inflammatory genes (Tnfa, I/1b) in total monocytes ( $\mathrm{N}=4 /$ group). (L) The expression of anti-inflammatory genes ( $\mathrm{Ym} 1$ and Tgfbi) in total monocytes ( $N=4 /$ group). ${ }^{*} p<0.05,{ }^{* *} p<0.01$. Results are represented as median with $25^{\text {th }}$ and $75^{\text {th }}$ percentiles, capped bars at $10^{\text {th }}$ and $90^{\text {th }}$ percentile and compared between HFD L $f^{\text {fl/fl }}$ and HFD i $L \mathrm{pH}^{--}$using unpaired t-test. 

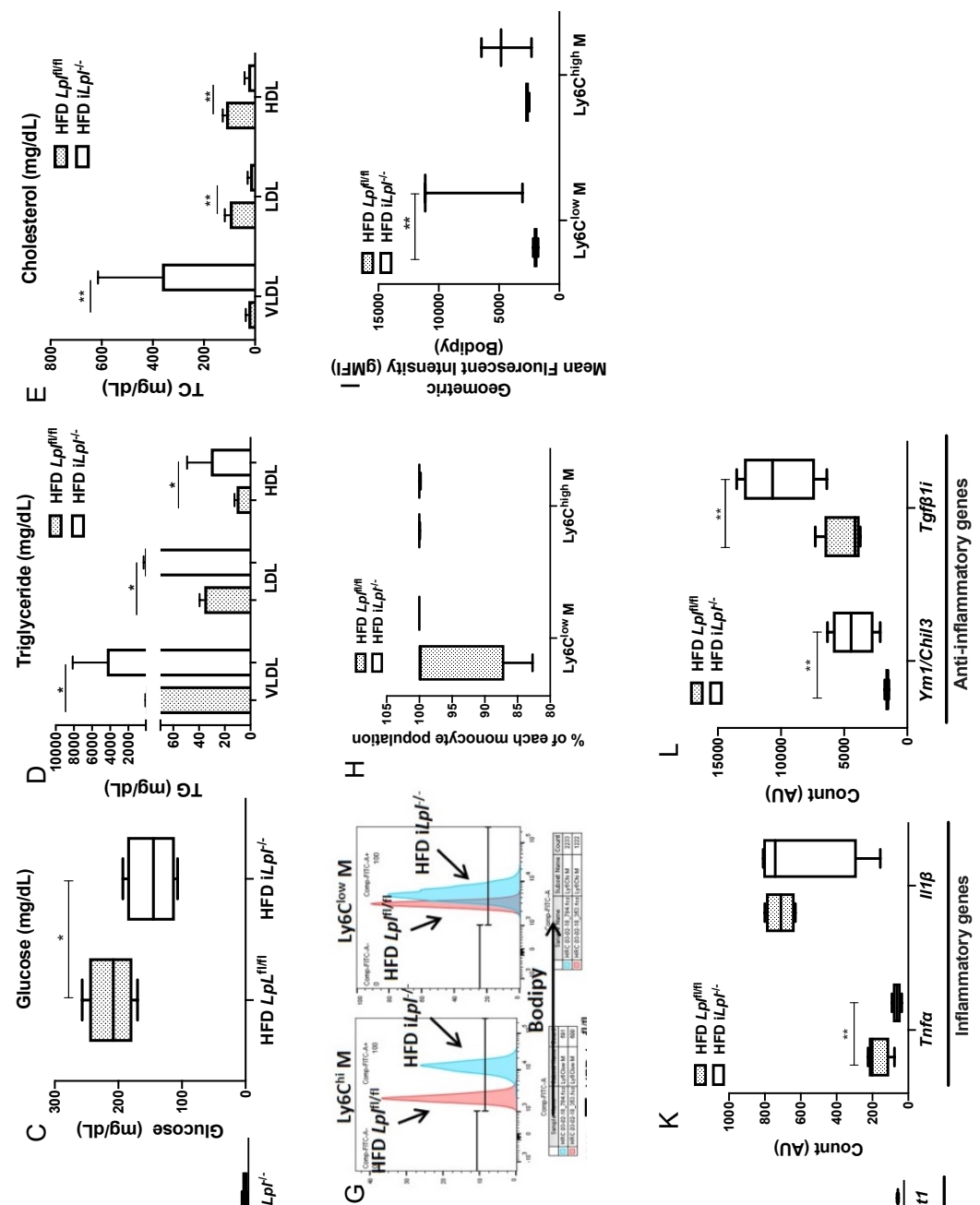

丧
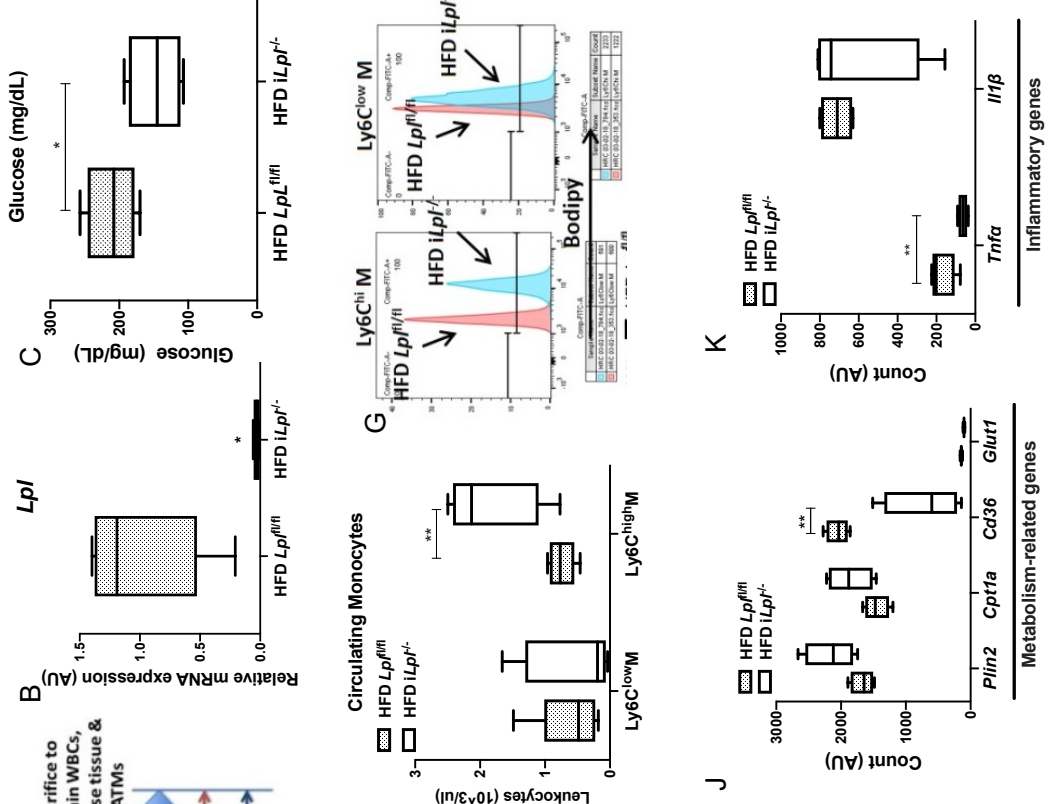

음
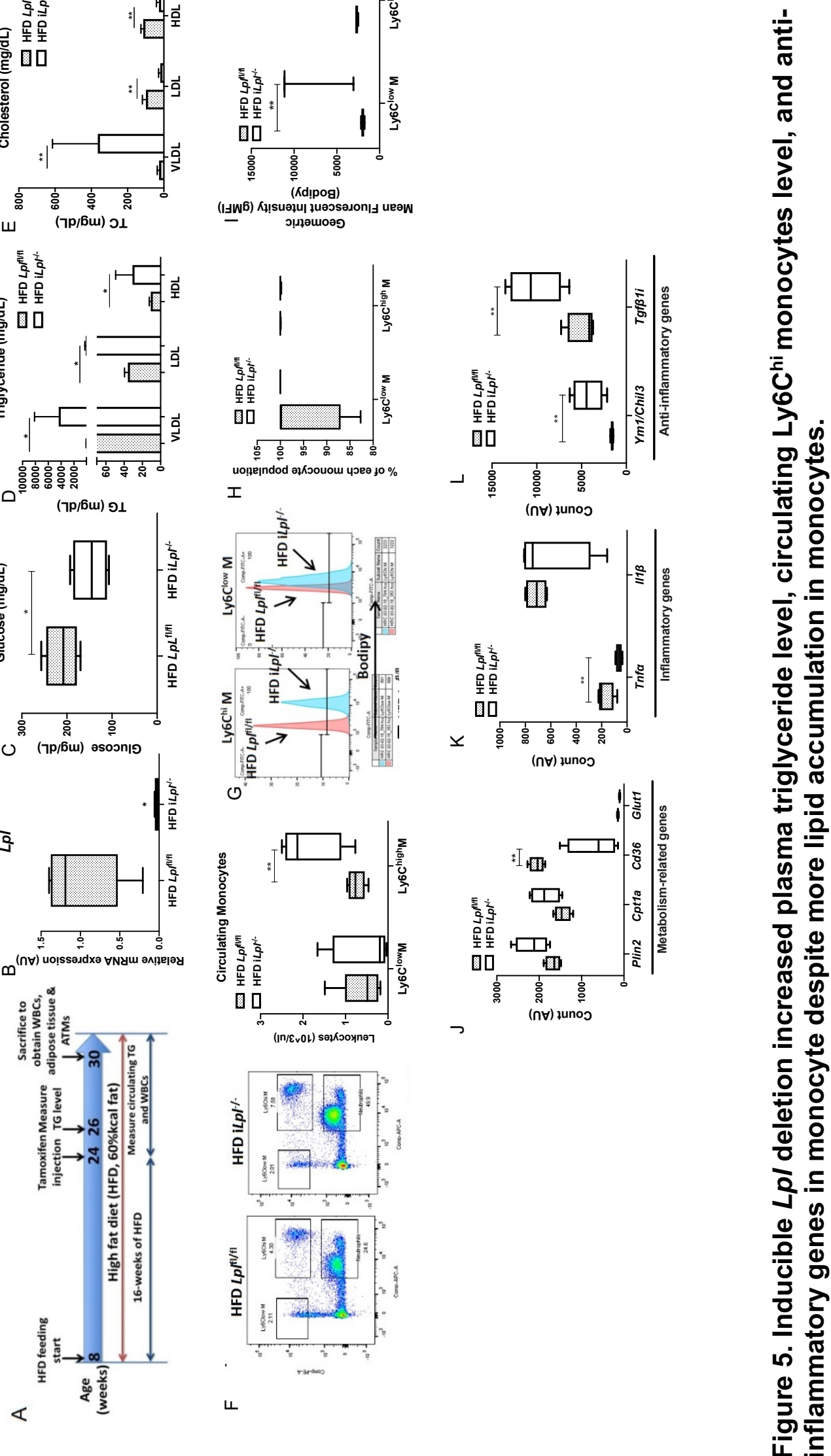


\section{The effect of inducible $L p /$ deficiency in adipose tissue macrophages in obesity:}

Because myeloid cell-derived-specific $L p /$ deficiency did not alter ATM phenotype, we postulated that this is due to developmental compensation and early action of LysMCre. We used $i \mathrm{Lpl}^{H^{-}}$mice to determine whether deleting $L p l$ after inducing obesity affects ATM subpopulation, lipid content, and phenotype. ATM $L p /$ expression was decreased $90 \%$ in $\mathrm{iLp}^{-1-}$ mice, however, total ATM (F4/80+) content did not differ between control and HFD i $\mathrm{Fp}^{-1-}$ mice (Figure $6 \mathrm{~A}$ ). With regard to subpopulations, percent of $\mathrm{FBs}$ from total $\mathrm{F} 4 / 80$ + cells was significantly higher, whereas FBCs from

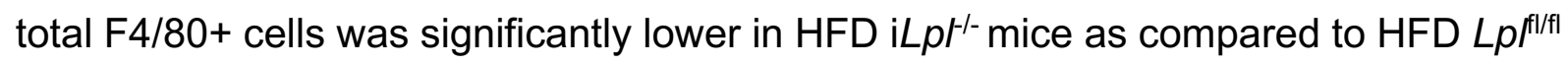
mice (Figure 6B, 6C). This suggests that $L p L$ is necessary for lipid accumulation in ATMs and important for FBC ATM development in obesity. To further examine whether Lpl deletion affects lipid content in ATMs, we measured neutral lipid content in both FBs and FBCs. Intracellular neutral lipid accumulation measured by percentage of bodipy+ cells and bodipy+ gMFI in FBs and FBCs in HFD i $L p^{H-}$-mice tended to be lower in HFD iLpH- mice despite the marked hypertriglyceridemia in these mice (Figure 6D, $6 \mathrm{E}$, and $6 \mathrm{~F}$ ). These data show that lack of $L p /$ in adipocytes decreases quantity of FBCs and prevents lipid accumulation in ATMs.

To examine whether LpL affects ATM phenotype, we measured both inflammatory and anti-inflammatory gene expression. Although Glut1 gene expression was dramatically increased in total ATMs of HFD iLpr-- mice (Figure 6G), mRNA levels of inflammatory genes (II1 $\beta$, Tnfa) tended to be lower (Figure $6 \mathrm{H}$ ). One anti-inflammatory gene, $Y \mathrm{Ym}-1$, increased, but other M2 genes (Arg-1 and Fizz-1) were unaltered (Figure 5E and 6I) in total ATMs. A FAO-related gene, Cpt1a, and other lipid-related genes (Cd36, Plin2) were not affected (Figure $6 G$ ). Overall, our data show that although lack of $L p L$ reduced the number of lipid-laden ATMs and appeared to shift M $\phi s$ to a more glucose-oxidizing phenotype (increased Glut1 mRNA level), this phenomenon did not lead to M1 phenotype in ATMs. 


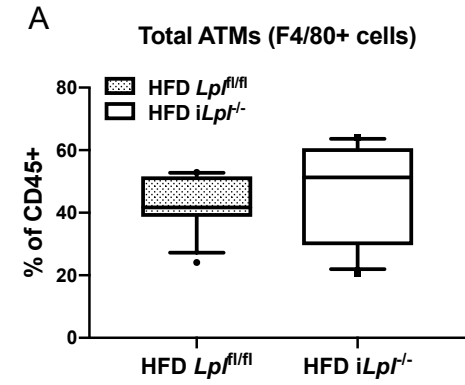

$\mathrm{D}$

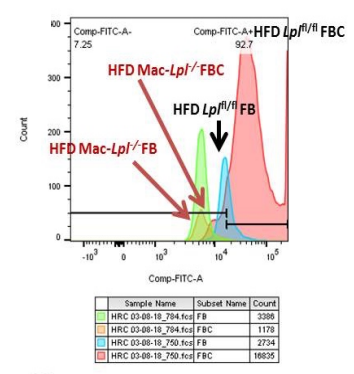

G

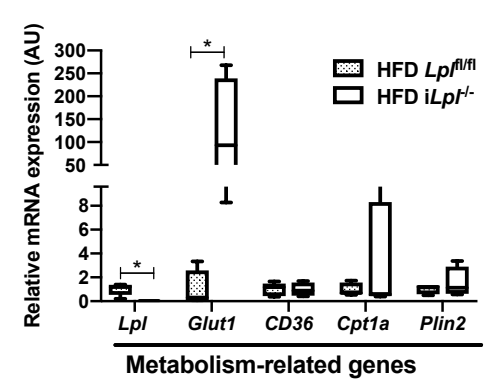

B

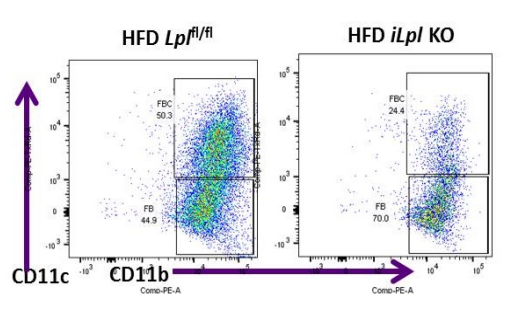

E

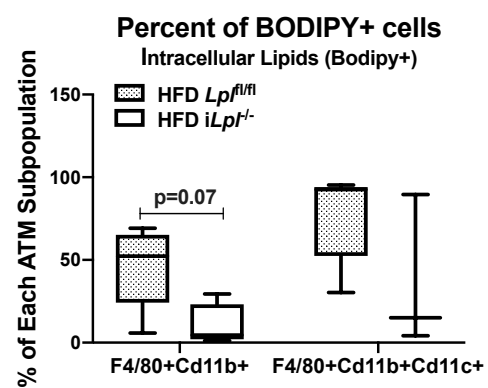

$\mathrm{H}$

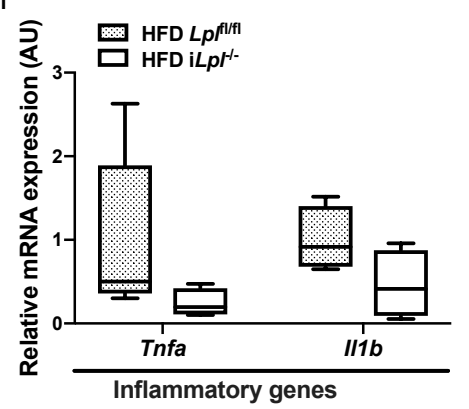

C

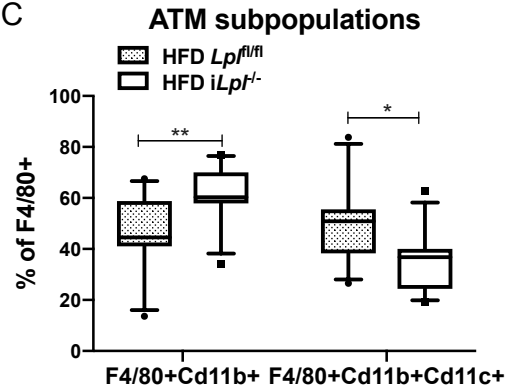

$\mathrm{F}$
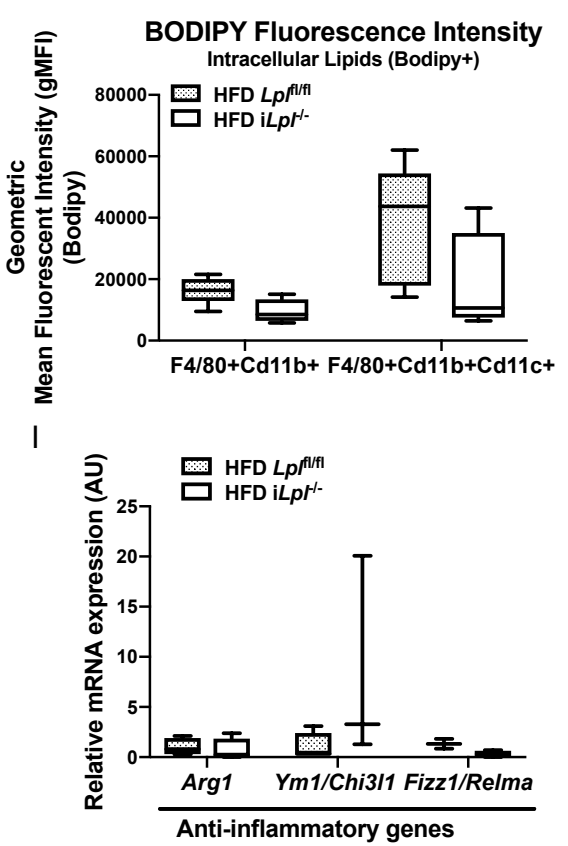

Figure 6. Inducible $L p l$ ablation decreases CD11c+ ATMs (FBCs) content, ATM lipid accumulation, but does not affect ATM polarity despite a dramatic increase

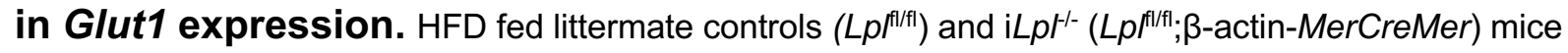
were studied for total ATM (CD45+F4/80+) content, ATM subpopulation (CD45+F4/80+Cd11b+ and $\mathrm{CD} 45+\mathrm{F} 4 / 80+\mathrm{CD} 11 \mathrm{~b}+\mathrm{CD} 11 \mathrm{c}+$ ), and ATM polarity. (A) Flow cytometry analysis of total ATMs (CD45+F4/80+). (B) Representative flow cytometry plots for ATM subpopulations; FBs $(C D 45+F 4 / 80+C d 11 b+)$ and FBCs $(C D 45+F 4 / 80+C D 11 b+C D 11 c+)$ and quantified FBs and FBCs ( $N=11-17 / g r o u p)$. (C) Quantified percentage of $F B s$ and $F B C s$ from $C D 45+F 4 / 80+$ cells. (D) Representative flow cytometry histogram plots are shown for BODIPY fluorescence in FBs and FBCs from HFD $L p^{f / / f l}$ and HFD i $L p r^{- \text {- }}$ mice (N=3-5/group). (E) Quantified percentage of BODIPY fluorescence in FBs and $\mathrm{FBCs}(\mathrm{N}=3-5 /$ group). ( $\mathrm{F})$ Quantification of BODIPY geometric mean fluorescence intensity (gMFI) in FBs and FBCs (N=3-5/group). (G) The expression of metabolism-related genes (Lpl, Glut1, Cd36, Cpt1a, Plin2) in ATMs (N=4-5/group). (H) The expression of inflammatory genes (Tnfa, I/1b) in ATMs ( $\mathrm{N}=4-5 /$ group). (I) The expression of anti-inflammatory genes (Arg1, Ym1, Fizz1) in ATMs ( $\mathrm{N}=4-$ $5 /$ group). ${ }^{*} p<0.05,{ }^{* *} p<0.01$. Results are represented as median with $25^{\text {th }}$ and $75^{\text {th }}$ percentiles, capped 
bars at $10^{\text {th }}$ and $90^{\text {th }}$ percentile and compared between $L p f^{f / / f l}$ and $i L{ }^{-/-}$using unpaired t-test $(A-F, C D 36$, Tnfa, I/1b) or Mann-Whitney Test (LpL, Glut1, Cpt1a, Plin2).

The impact of myeloid cell-specific $L p /$ deletion in peritoneal $\mathbf{M} \phi \mathrm{s}$ : Tissue-specific M $\phi$ s have distinct phenotypes and functions, depending on the tissue microenvironment. A previous report demonstrated that VLDL-derived FA uptake induces inflammation in peritoneal $M \phi s$ in vitro, suggesting that $L \mathrm{pL}$-dependent lipid uptake is inflammatory ${ }^{13}$. We next assessed whether $L p L$ affects peritoneal $M \phi$ number and polarity, both in resident $\left(\mathrm{F} 4 / 80^{\text {hi }} \mathrm{MHCll}{ }^{\text {low }}\right)$ and recruited $\left(\mathrm{F} 4 / 80^{\text {low }} \mathrm{MHCl}{ }^{\text {high }}\right)$ populations. We induced $\mathrm{M} \phi$ recruitment using zymosan $\mathrm{A}$ (100 $\mu \mathrm{g} /$ mouse; Sigma) in mice fed a normal rodent diet, as shown in Figure 7A. We found that lack of $\mathrm{LpL}$ did not affect $\mathrm{M} \phi$ accumulation in the peritoneal cavity (Figure 7B, 7C, and 7D).
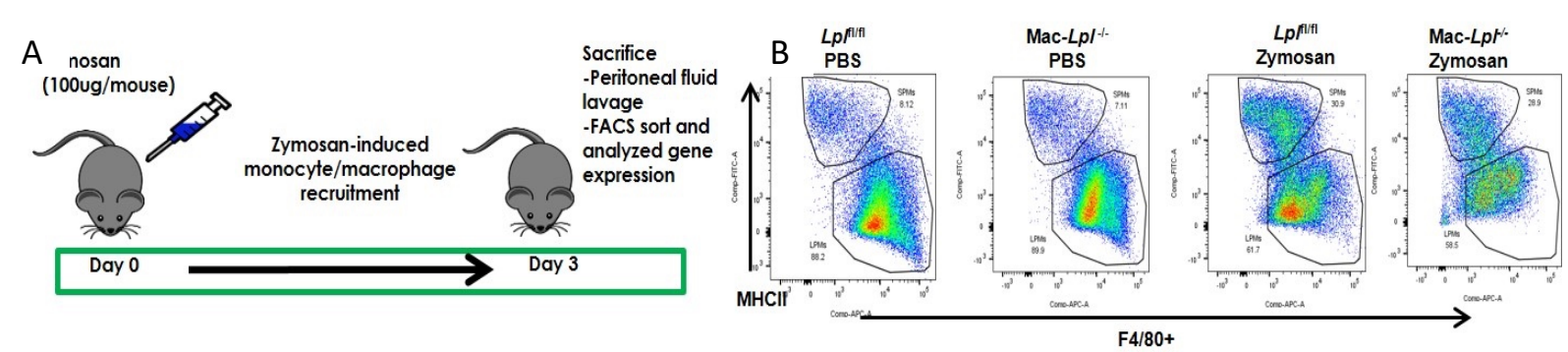

C Resident Peritoneal Mac (F4/80 ${ }^{\text {himHCIIlow) }}$
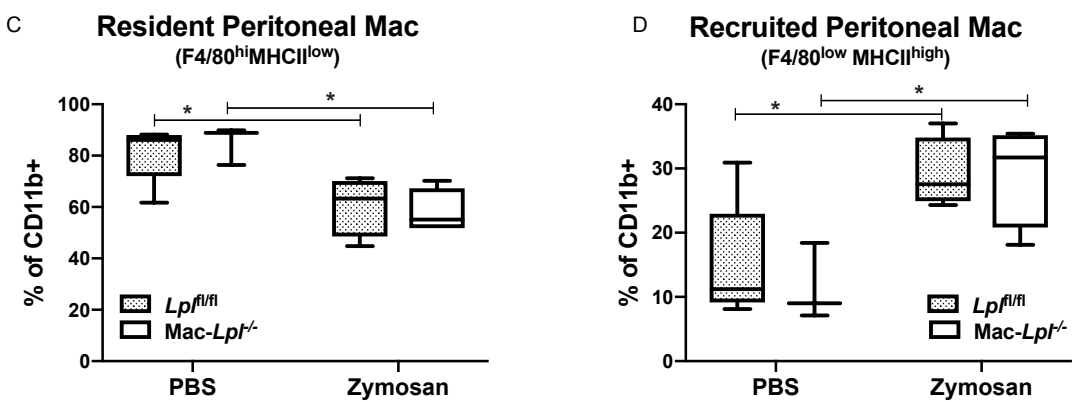

Figure 7. Myeloid-cell derived $L p /$ deficiency does not affect number of

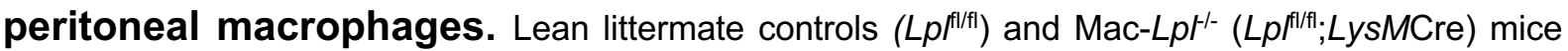
were studied for both resident peritoneal macrophages $\left(\mathrm{F} 4 / 80^{\text {high }} \mathrm{MHCl}{ }^{\text {low }}\right)$ and recruited peritoneal macrophages ( $\left.\mathrm{F} 4 / 80^{\text {low }} \mathrm{MHCI}{ }^{\text {high }}\right)$ under zymosan-induced peritonitis. (A) Experimental design:

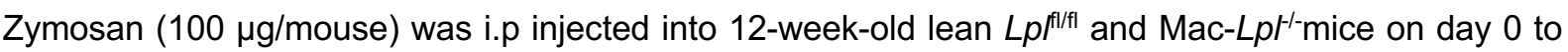
induce monocyte/macrophage recruitment to peritoneal cavity. All mice were sacrificed on day 3 for peritoneal macrophage analysis using flow cytometry. (B) Representative flow cytometry plots for peritoneal macrophages subpopulations. (C) Flow cytometry analysis of resident peritoneal macrophages. (D) Flow cytometry analysis of recruited peritoneal macrophages. $\mathrm{N}=3-5 /$ group. ${ }^{*} \mathrm{p}<0.05$. 
Results are represented as median with $25^{\text {th }}$ and $75^{\text {th }}$ percentiles, capped bars at $10^{\text {th }}$ and $90^{\text {th }}$ percentile and compared using Two-Way ANOVA with Sidak's multiple comparison test.

Glut1 mRNA expression was also significantly up-regulated in Mac-Lp ${ }^{-1-}$ LPMs as compared to $L p f^{f / f / f l} L P M s$ (Figure 8B), indicating that genetic ablation of LpL may lead to a metabolic reprogramming. However, despite an increase in Glut1, both inflammatory and anti-inflammatory genes were not significantly different in LPMs between Mac- $L p I^{-1-}$ and $L p f^{f / / 1 / 1}$ mice (Figure 8E-8I). These results show that independent of hyperlipidemic conditions, $L \mathrm{~L}$ deficiency seems to shift the energy source from FA to glucose, as measured by Glut1 expression, this metabolic reprogramming does not impact M1/M2 phenotype of tissue M $\phi s$ both in AT and the peritoneal cavity.
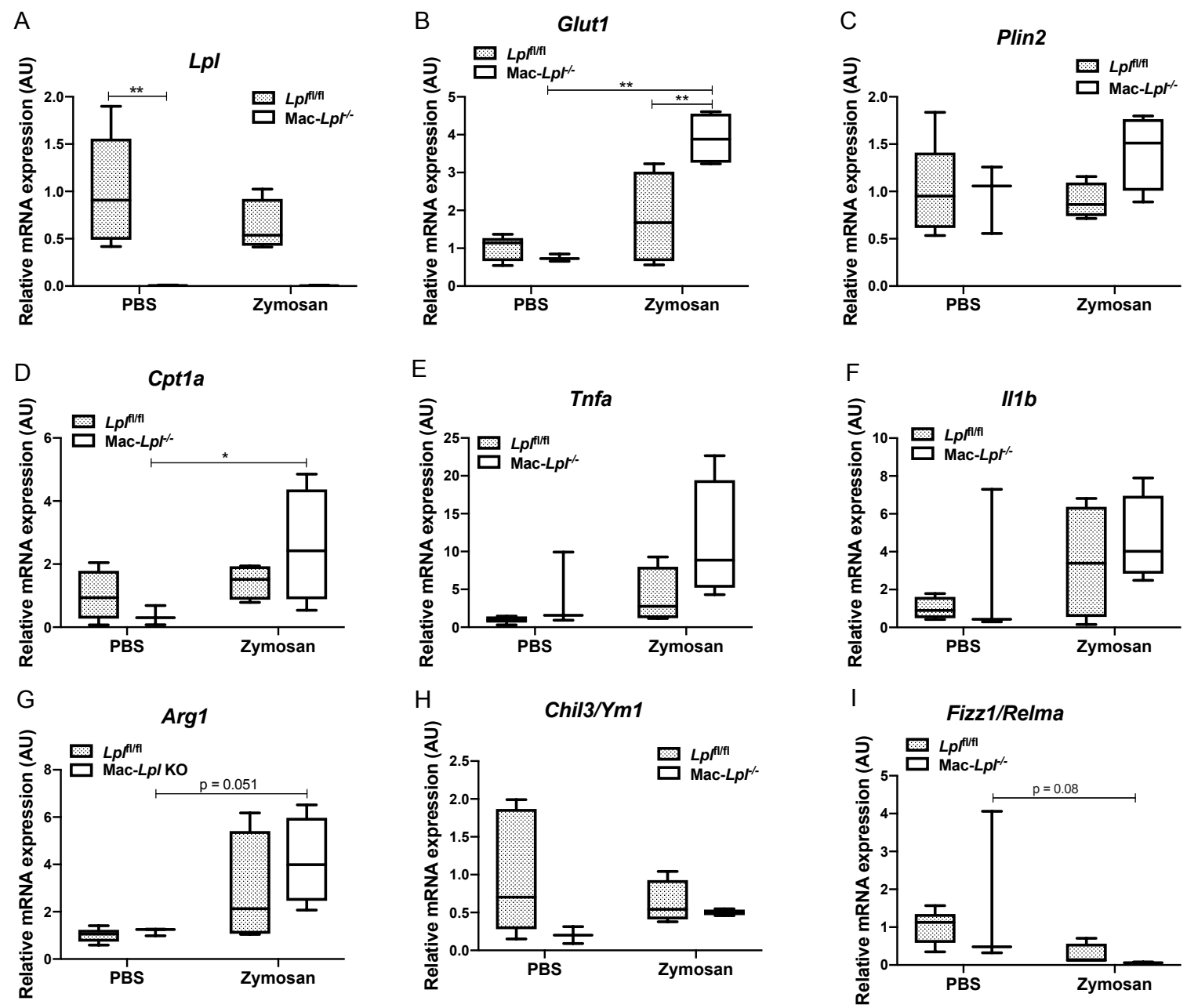

Figure 8. Myeloid-cell derived $L p /$ deficiency increases metabolic genes, but does not profoundly affect canonical inflammatory and anti-inflammatory genes in resident peritoneal macrophages under zymosan stimuli. (A) The expression of $L p l$ in resident peritoneal macrophages ( $\left.\mathrm{Cd} 45^{+} \mathrm{F} 4 / 80^{\text {high }} \mathrm{MHCll}{ }^{\text {low }}\right)$. (B) The expression of Glut1 in resident 
peritoneal macrophages. (C) The expression of Plin2 in resident peritoneal macrophages. (D) The expression of Cpt1a in resident peritoneal macrophages. (E) The expression of Tnfa in resident peritoneal macrophages. (F) The expression of $\| 11 b$ in resident peritoneal macrophages. (G) The expression of Arg1 in resident peritoneal macrophages. $(\mathrm{H})$ The expression of $Y m 1$ in resident peritoneal macrophages. (I) The expression of Fizz1 in resident peritoneal macrophages. N=3-5/group. ${ }^{*} p<0.05,{ }^{* *} p<0.01$. Results are represented as median with $25^{\text {th }}$ and $75^{\text {th }}$ percentiles, capped bars at $10^{\text {th }}$ and $90^{\text {th }}$ percentile and compared using Two-Way ANOVA with Sidak's multiple comparison test.

The effect of inducible $L p /$ deficiency in peritoneal $M \phi s$ : In contrast to Mac- $L p{ }^{-1-}$ mice, there was more than a $60 \%$ decrease in both resident and recruited peritoneal $\mathrm{M} \phi \mathrm{s}$ in i $L p \mathrm{~F}^{-/}$mice as compared to those in $L \mathrm{p}^{\mathrm{fff}}$ mice (Figure 9B, 9C, 9D). The dramatic reduction in the resident population was evident even in the control (PBS treatment) group, showing that $L p L$ is required for maintaining the tissue resident $M \phi s$ in the cavity.
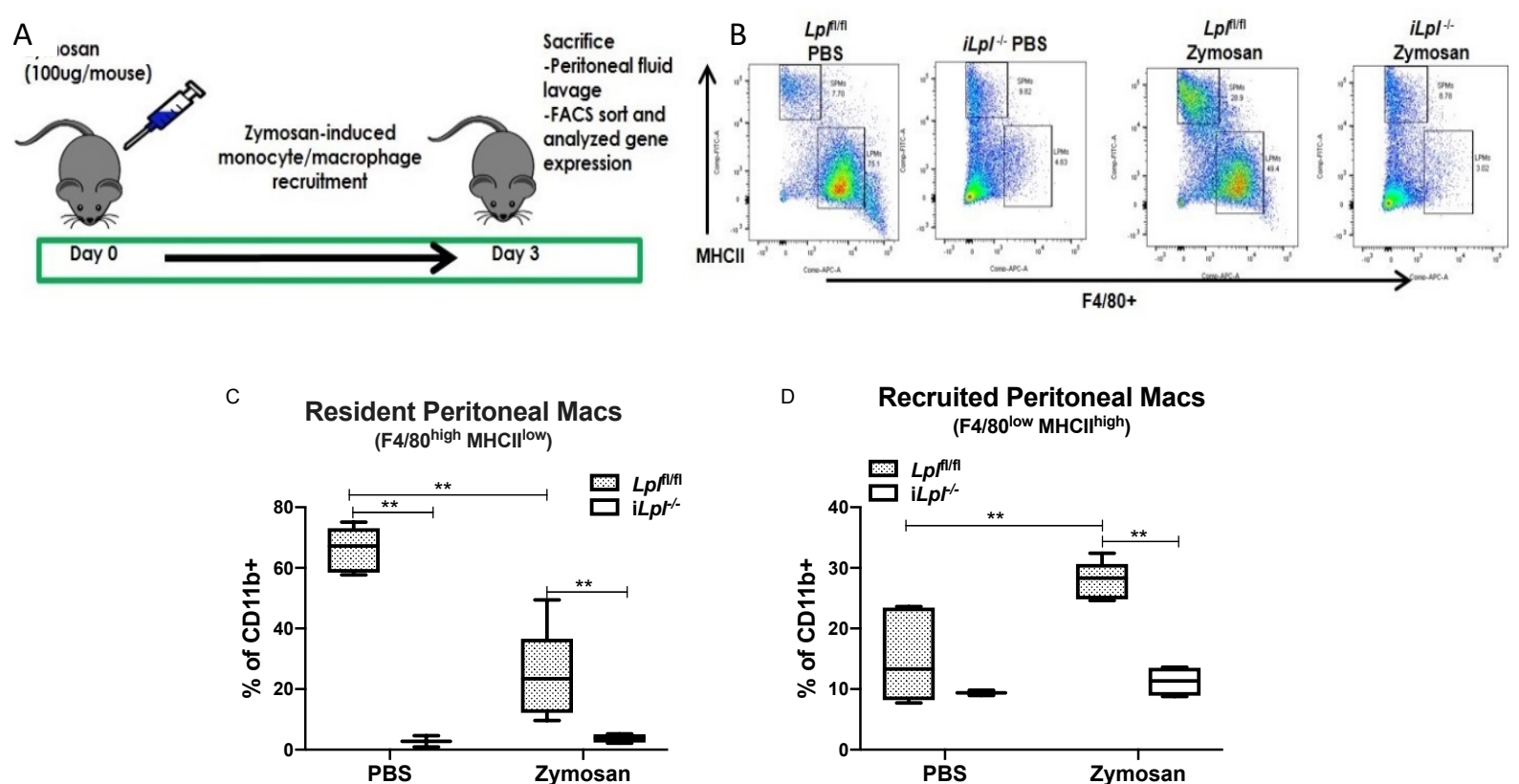

Figure 9. Inducible Lpl deletion dramatically decreases both resident and recruited peritoneal macrophages content upon zymosan stimuli. Lean littermate

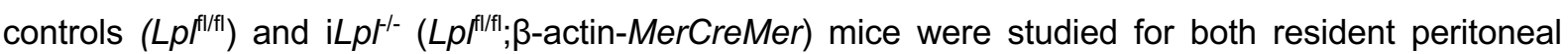
macrophages ( $\left.\mathrm{F} 4 / 80^{\text {high }} \mathrm{MHCI}{ }^{\text {low }}\right)$ and recruited peritoneal macrophages ( $\mathrm{F} 4 / 80^{\text {low }} \mathrm{MHCl}$ high $)$ under zymosan-induced peritonitis. (A) Experimental design: Zymosan (100 $\mu \mathrm{g} / \mathrm{mouse})$ was i.p injected into

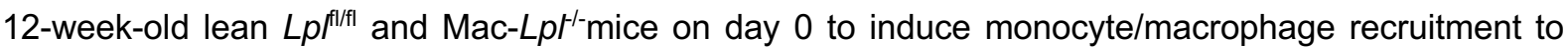
peritoneal cavity. All mice were sacrificed on day 3 for peritoneal macrophage analysis using flow cytometry. (B) Representative flow cytometry plots for peritoneal macrophages subpopulations. (C) Flow cytometry analysis of resident peritoneal macrophages. (D) Flow cytometry analysis of recruited 
peritoneal macrophages. $N=3-5 /$ group. ${ }^{*} \mathrm{p}<0.05$. Results are represented as median with $25^{\text {th }}$ and $75^{\text {th }}$ percentiles, capped bars at $10^{\text {th }}$ and $90^{\text {th }}$ percentile and compared using Two-Way ANOVA with Sidak's multiple comparison test.

These results are parallel to those found with angiopoietin-like protein 4 deficiency where greater $\mathrm{LpL}$ activity is associated with severe peritoneal inflammation ${ }^{44}$. The decrease in recruited population illustrates that $L p L$ is also important in monocyte recruitment into the peritoneal cavity upon interaction with inflammatory stimuli such as zymosan. While we were analyzing the M1/M2 gene expression profile from the resident and recruited peritoneal $M \phi s$, we were unable to obtain an optimum amount of RNA to measure those genes due to the low number of resident peritoneal $M \phi s$ (LPMS). The gene expression profile from the recruited population (SPMs), however, was analyzed. We found a pattern of increase in Glut1 expression (Figure 10B) along with significant Tnfa and $I / 1 b$ expression (Figure 10E) in $\mathrm{i} L \mathrm{~F}^{-{ }^{-}}$mice relative to $L p f^{f / f l}$ mice. When we analyzed M2 genes, Arg1 was significantly upregulated in i $\mathrm{Lp}^{-/}$mice compared to $L p f^{f f l}$ mice, but not $Y m 1$ and Fizz1 (Figure 10G, 10H, 10I). Because we measured the gene expression in the recruited population, it is difficult to make a parallel comparison with the results from the Mac-Lph ${ }^{-1}$ mice. However, the gene expression profile from total peritoneal $M \phi s$ was identical to the gene expression from the recruited population (Supplemental Figure 9). These data show that $L p L$ derived from non-myeloid cells is required for $M \phi$ development, recruitment, and polarization in peritoneal cavity. 

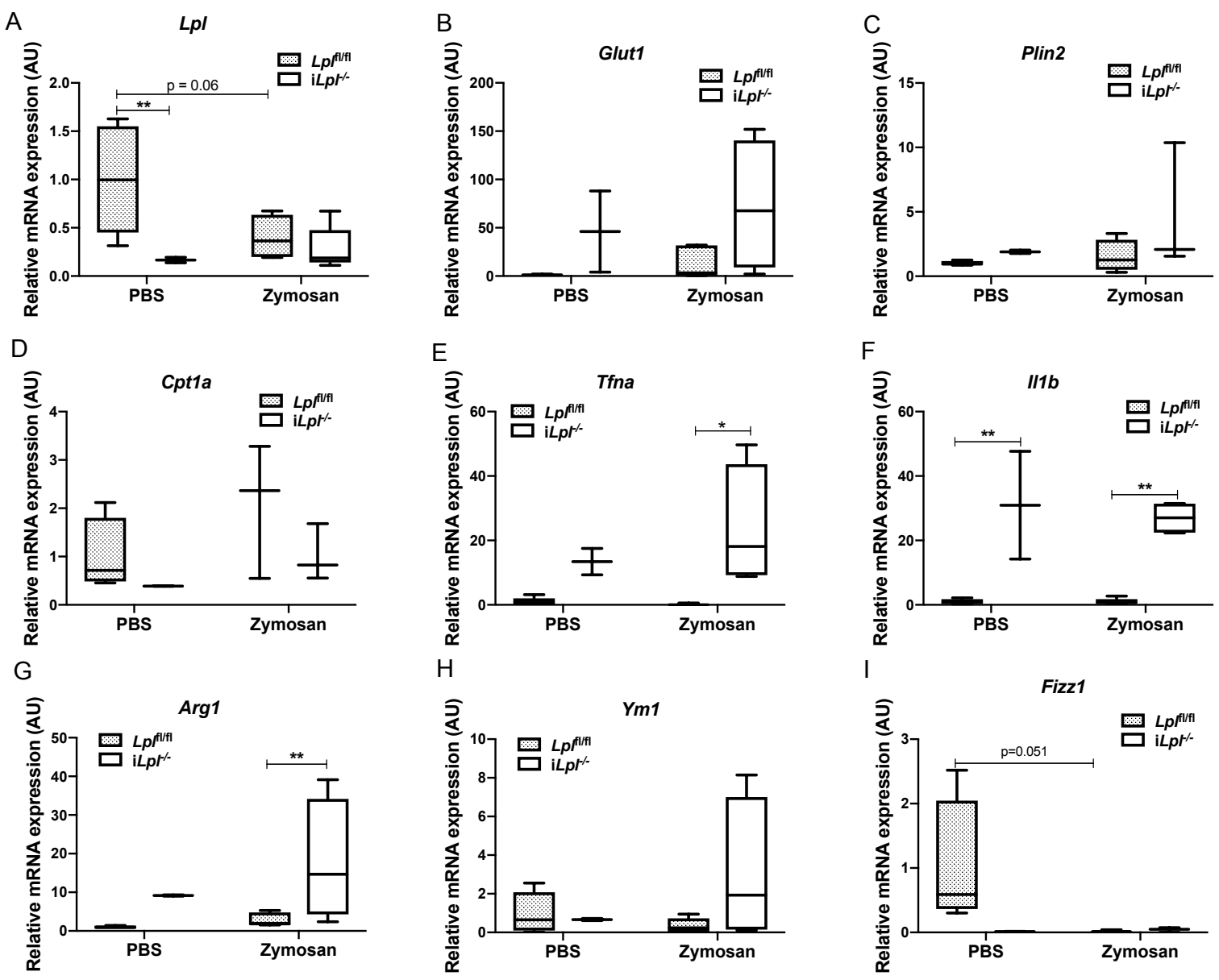

Figure 10. Inducible Lpl ablation increases metabolic-, inflammatory-, and antiinflammatory genes in recruited peritoneal macrophages upon zymosan stimuli.

(A) The expression of $L p l$ in recruited peritoneal macrophages $\left(\mathrm{Cd} 45^{+} \mathrm{F} 4 / 80^{\text {low } M H C l{ }^{\text {high }}}\right)$. (B) The expression of Glut1 in resident peritoneal macrophages. (C) The expression of Plin2 in resident peritoneal macrophages. (D) The expression of Cpt1a in resident peritoneal macrophages. (E) The expression of Tnfa in resident peritoneal macrophages. (F) The expression of $/ 11 \mathrm{~b}$ in resident peritoneal macrophages. (G) The expression of Arg1 in resident peritoneal macrophages. $(\mathrm{H})$ The expression of Ym1 in resident peritoneal macrophages. (I) The expression of Fizz1 in resident peritoneal macrophages. $N=3-5 /$ group. ${ }^{*} p<0.05,{ }^{* *} p<0.01$. Results are represented as median with $25^{\text {th }}$ and $75^{\text {th }}$ percentiles, capped bars at $10^{\text {th }}$ and $90^{\text {th }}$ percentile and compared using Two-Way ANOVA with Sidak's multiple comparison test.

The impact of inducible $L p l$ deletion on atherosclerosis plaque $M \phi s$ in the context of regression: We hypothesized that lack of $M \phi$-derived $L p L$ would prevent the polarization of newly recruited $\mathrm{M} \phi$ s to $\mathrm{M} 2$ and negatively affect regression. To test our hypothesis, we utilized a well-established aortic transplant methodology 28,29 to 
create a plaque regression microenvironment in $L p f^{f l / f l}$ and $i L{ }^{H^{--}}$mice as described in Figure 11A. Two weeks after inducing regression, we isolated CD68+ Mфs from plaques using laser capture microdissection 26,45 and measured gene expression. Consistent with the other tissue M $\phi \mathrm{s}, L p /$ deletion increased Glut1 mRNA levels (Figure $11 \mathrm{~B} ; \mathrm{p}=0.08$ ). Similar to in vivo ATMs, the expression of Plin2, Cd36, and Cpt1a genes were not altered in iL $\mathrm{pl}^{-{ }_{-}}$mice. Although we found a pattern of decrease in Mcp1 mRNA level in $i L p H^{-1-}$ mice as compared to $L p f^{f l / f l}$ mice, unaltered expression of other typical inflammatory genes (Tnfa, Ilb and Nos2) was observed (Figure 11C). In contrast to our hypothesis, the anti-inflammatory genes (Mrc1, Fizz1, //10) were not significantly decreased upon deletion of $L p l$ (Figure 11D). We confirmed our gene expression results for mannose receptor Mrc1/CD206, a representative M2 marker in mouse and human plaque $M \phi s^{4}$, by CD206 immunostaining in the regressing plaques. The quantified area of mannose receptor in $i L p I^{--}$mice was comparable with $L p f^{f / / f l}$ mice (Figure 11E), indicating that $\mathrm{LpL}$ does not affect plaque $M \phi M 2$ polarization in regression. As expected, $L p /$ deficiency had no impact on regressing plaque area (Figure 11F). Of note, despite plaque area not having changed in the regression groups compared to baseline, amount of CD68+ cells were reduced, representing atherosclerosis regression (data not shown). Overall, we found that LpL is dispensable for M2 polarization in plaque M $\phi s$ and resolution of atherosclerosis.

Figure 11: (A) Experimental design: A plaque burden aortic arch from $L d l r^{\prime-}$ mice was transplanted into the 22-week-old recipient mice (i $L p^{H^{--}}$or $L p^{f / / f l}$ mice, maintained on a standard laboratory diet), interpositioned with the abdominal aorta, and blood flow was directed through the graft. All mice were sacrificed 14 days after the aortic arch transplantation. (B) The expression of metabolism-related genes (Lpl, Glut1, Plin2, Cd36, Fsn, Cpt1a) in plaque macrophages. (C) The expression of inflammatory genes (Tnfa, I11b, Nos2, Mcp1[Cc/2]) in plaque macrophages. (D) The expression of anti-inflammatory genes (Mrc1, Fizz1, I/10) in plaque macrophages. (E) Quantification of mannose receptor (Cd206) staining in the atherosclerotic plaque (as shown with area unit, $u^{2}$, and percent of the plaque size per field). $(F)$ Quantification of plaque area. (G) Representative images for the co-localization of CD68+ and CD206+ staining and the quantification of double-positive $(C D 206+C D 68+)$ cells. $N=4-7 /$ group (baseline $n=4$, $\left.L p f^{f / / f l} n=7, i L p H^{-/} n=7\right) .{ }^{*} p<0.05$. Results are represented as median with $25^{\text {th }}$ and $75^{\text {th }}$ percentiles, capped bars at $10^{\text {th }}$ and $90^{\text {th }}$ percentile and compared using unpaired $t$-test $(L p l$, Plin2, Glut1, Cd36, Nos2, Mcp1, Fizz1/Relma, IL-10), Mann-Whitney test (Fasn, Cpta1, Mrc1), Welch's t-test (II1b, Tnfa), or One-way ANOVA with Tukey's multiple comparison (E, F). 
Chapter 3

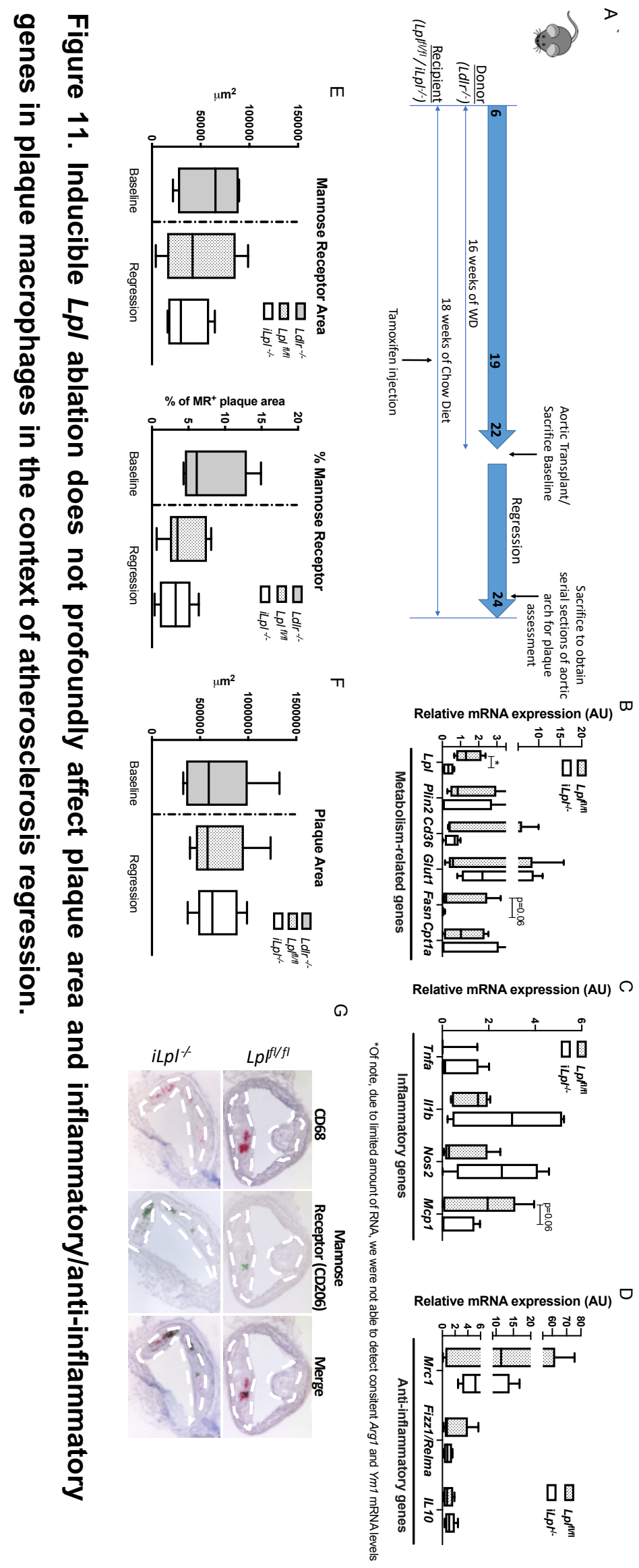




\section{Discussion}

Genetic ablation of $L p /$ in vitro and/or in vivo demonstrated distinct effects on $M \phi$ phenotype. It is widely accepted that $M \phi$ phenotype is associated with, and likely depends on, cellular differences in lipid and glucose metabolism ${ }^{3,46}$. Moreover, several in vitro studies indicate that $L \mathrm{pL}$ is a primary regulator of $M \phi$ lipid uptake ${ }^{14}$ and therefore a modulator of $M \phi$ polarity ${ }^{5,19}$. By comparing the role of this enzyme both in vitro and in in vivo, our data challenge these assumptions. We show that deleting myeloid cell-derived LpL does not alter M $\phi$ polarity in obese AT, peritoneal cavity, and regressing atherosclerotic plaques. In all depots, cellular lipid metabolism does not correlate with $M \phi$ phenotype, and the results support a model in which tissue environment plays the central role in determining how subsets of $M \phi s$ respond to changes in ability to produce FFAs from TGs.

Although we were able to create a TG-rich environment both in the circulation and in AT by feeding the mice a HFD, we also created a robust inflammatory microenvironment with high concentrations of glucose, insulin, and FFAs-so called metabolic chronic inflammation. In a human monocyte cell line (THP-1), LpL-mediated VLDL uptake induces both intracellular lipid accumulation and inflammation, as measured by $I I 1 \beta$ and Tnfa mRNA expression ${ }^{17}, 18$. However, in vivo monocytes, markers of metabolism and phenotype were unchanged in Mac- $L \mathrm{pl}^{-/-}$mice, indicating that the inflammatory microenvironment induced by a HFD in vivo adapted to compensate for the lack of LpL in monocytes. In ATMs, local FFA concentrations are likely higher than FFAs in systemic circulation due to the close proximity to adipocyte lipolysis. Thus, our findings in ATMs in Mac- $\mathrm{LP}^{-{ }^{-}}$mice-unaltered ATM subpopulation, lipid accumulation, and phenotype-are likely due to FFA uptake via FA transporters or the flip-flop pathway, endocytic TG-rich lipoprotein uptake, or accumulation of adipocyte-derived lipid vesicles ${ }^{47}$. This may explain the lack of changes in inflammatory gene expression phenotype in Mac- $\mathrm{Lp}^{H_{-}-}$mice despite increased Glut1 mRNA expression in ATMs.

Another possible interpretation is that since $L y s M$-Cre is effective starting from the development stage, ablation of $L \mathrm{pL}$ may have induced a compensatory mechanism in the early developmental stages of mice. We tested the latter hypothesis by using HFD $\mathrm{i} L \mathrm{pl}^{-1-}$ mice. We found a significant increase in levels of circulating Ly6C $\mathrm{C}^{\text {hi }}$ monocytes with a significant increase in lipid accumulation within Ly6 $\mathrm{C}^{\text {low }}$ monocytes. Because we 
did not find increased LDL receptor (Ldlr), VLDL receptor (VIdlr) (data not shown), or Glut1 gene expression level, higher neutral lipid content in Ly6Clow monocytes in HFD $\mathrm{i} L \mathrm{pH}^{-1-}$ mice is perhaps due to LpL-independent lipid uptake pathway such as FFA uptake via the flip-flop pathway, as serum FFA concentration was higher in HFD iL $\mathrm{F}^{-{ }^{-}}$ mice than in HFD $L p^{f / f l}$ mice (Supplementary Figure $4 G$ ). Interestingly, although levels of circulating Ly6 $6 \mathrm{C}^{\text {hi }}$ monocytes were higher in HFD i $L \mathrm{pr}^{-/}$mice, a typical inflammatory gene, Tnfa gene expression, was lower, but anti-inflammatory genes were higher as compared to HFD $L p f^{f / f f l}$ mice. These results show that, as previously shown by Fisher and Pearce groups ${ }^{4,48}$, Ly6C ${ }^{\text {hi }}$ monocytes have potential to become M2 M $\phi$ s.

Even though inducible LpL deletion in obese mice did not affect total ATM content $(\mathrm{F} 4 / 80+$ cells), it shifted the FB:FBC ratio. Obesity is associated with FBC populations as they accumulate more neutral lipids ${ }^{24}$, however, deleting $L p l$ in obese mice decreased FBCs and increased FBs. There was a pattern of a reduction in intracellular lipid content in FBCs and FBs $(p=0.07)$ in HFD $i L p H^{-1}$ mice, as compared to ATMs in HFD $L p^{f / / f l}$ mice. In contrast, ATMs in HFD Mac-Lpl-- mice and HFD $L \mathrm{p}^{\mathrm{fl/fl}}$ mice showed no difference in intracellular lipid content. One explanation for this is that lipid accumulation in $M \phi s$ is dependent on adipocyte-derived $L \mathrm{pL}$. Another explanation is that uptake requires systemic initial lipolysis of TG-rich lipoproteins by LpL to allow their internalization by the ATMs, e.g. via lipoprotein receptors. Lipoprotein receptor uptake of nascent TG-rich lipoproteins requires their partial lipolysis to remnant or intermediate density lipoproteins to allow optimal uptake via lipoprotein receptors. Total deficiency of $L \mathrm{pL}$ will eliminate these particles and might be the reason that systemic rather than $M \phi$ specific $L p L$ deficiency reduced $M \phi$ lipid droplets. Such a conclusion would contrast with in vitro studies showing $M \phi$ lipid accumulation from chylomicrons via LpL actions.

Although Glut1 mRNA expression was dramatically increased in ATMs in HFD iLpl-mice, this did not affect the M1/M2 phenotype in ATMs, contradicting the current dogma of $M \phi$ polarization in vitro ${ }^{2}$. The dogma is that greater glucose utilization creates inflammatory M $\phi s$. Our studies do not support this association as causative, as increased Glut1 mRNA did not create a more inflammatory phenotype in LpL deficient M $\phi$ s. Our data are consistent with the Glut1 overexpression mouse model phenotype ${ }^{36}$, which did not show more inflammation in $\mathrm{M} \phi \mathrm{s}$. Moreover, our data support a recent finding where deletion of a key enzyme involved in FAO, carnitine 
palmitoyltransferase II (Cpt2), showed no effect on M2 conversion in both BMDMs and ATMs ${ }^{49}$. Overall, our data demonstrate that myeloid cell-derived LpL is dispensable for lipid uptake and polarity, however, LpL derived from other cells/tissues are perhaps necessary for ATM lipid accumulation.

The peritoneal cavity is a unique tissue compartment in both mice and humans. In addition to its distinctive anatomical structure, the peritoneal cavity contains many types of immune cells, such as lymphocytes, M $\phi s$, granulocytes, and mesothelial cells ${ }^{50}$, suggesting a complex cytokine profile in peritoneal fluid. Under normal physiological conditions, $91 \%$ of total myeloid cells $(\mathrm{Cd} 11 \mathrm{~b}+)$ are resident M $\phi$ s (LPMs), whereas $9 \%$ of total myeloid cells $(\mathrm{Cd} 11 \mathrm{~b}+)$ are recruited M $\phi s$ (SPMs) ${ }^{51}$. Upon a 3-day period of zymosan stimulation, the LPM:SPM ratio shifts from 91:9 to approximately 60:40. We sought to determine the role of LpL-mediated lipid uptake in both LPMs and SPMs in basal and zymosan-stimulated states. We found an increase in Glut1 and Plin2 mRNA levels in Mac- $L p \mathrm{~F}^{-/-}$mice under zymosan-stimuli in the resident population. These changes in metabolic-related gene expression are similar to the Mac- $L p^{H_{-}^{-}}$BMDMs cultured under low glucose media (Figure 1). When we measured glucose concentration in peritoneal cavity, it was low (below detection threshold of the glucometer), and TG concentrations were also lower than in the circulation (Supplemental Figure 8). Although the exact concentration of glucose in peritoneal cavity is unknown, our data suggest that the peritoneal cavity microenvironment is a low glucose milieu and $L p l$ deficiency in peritoneal M $\phi s$ induces metabolic compensation. However, these changes did not profoundly affect the inflammatory or anti-inflammatory state of the cells.

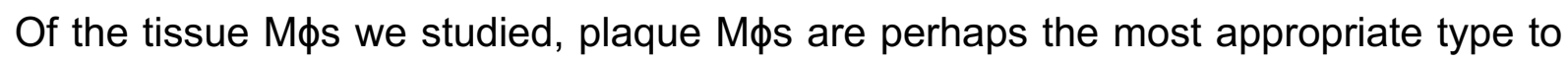
evaluate in vivo the role of $\mathrm{LpL}$ in both lipid metabolism and polarity, as the atherosclerotic lesion microenvironment offers immediate contact between $M \phi s$ and lipid particles. M $\phi$-expression of $L p L$ is atherogenic and associated with greater vascular inflammation ${ }^{21}$. The role of $M \phi$-derived $L \mathrm{pL}$ in regression, however, is yet to be defined. A recent study demonstrated that continued recruitment of inflammatory Ly6 $\mathrm{C}^{\text {hi }}$ monocytes and their polarization to the $\mathrm{M} 2$ state are required for the resolution of atherosclerotic inflammation and plaque regression ${ }^{4}$. This finding led us to question whether LpL has an impact on M2 polarization in plaque M $\phi$ s during plaque regression. We speculated that altering the most upstream pathway of lipid uptake by deleting LpL 
would affect alternative activation and resolution of atherosclerosis. However, our data show that ablation of LpL does not affect M2 conversion in plaque M $\phi$ s and thus does not change the plaque area. In fact, $L p /$ deficient $M \phi s$ from the regressing plaques had no change in the typical M2-related genes, including Mrc1 and Fizz1. This result is surprising because we observed a trend towards increased Glut1 mRNA levels in the plaque $M \phi s$, as we did in other in vivo $M \phi s$ and in vitro BMDMs. These findings again indicate a metabolic shift to glucose metabolism at least at the transcriptional level, but it neither led to an expression increase of inflammatory genes nor a decrease of antiinflammatory genes. The lack of impact on M2 conversion of plaque M $\phi s$ is perhaps due to multiple types of $M \phi s$ within the lesion area. Since atherosclerosis regression is a dynamic condition with constant recruitment of $L y 6 C^{\text {hi }}$ monocytes and retention of foam cells (well known as inflammatory), the baseline of regression condition is most likely comprised of a mix of M1/M2 M $\$$ s, as observed in human plaques ${ }^{52}$. Thus, deleting LpL may have affected both types of cells and led to no effect on the total plaque $M \phi s$ phenotype. It should be noted that, although we see a significant decrease in $\mathrm{LpL}$ expressing macrophages in $i \mathrm{~L}^{-/}$recipient mice, suggesting that the majority of $\mathrm{LpL}$ expressing macrophages from the donor has been replaced by recruited $\mathrm{Lp}^{-/}$ macrophages, we cannot exclude that there are LpL expressing macrophages left in the atherosclerosis lesions also affecting macrophage phenotype.

Overall, we discovered that, unlike previous in vitro studies, LpL-mediated FAs (PPARס ligands) are not required for $A A$ of $M \phi s$ in vivo. Our data clearly show that monocyte/tissue $M \phi$-derived $\mathrm{LpL}$ minimally regulates lipid accumulation in those cells in vivo and does not affect polarization. More importantly, $\mathrm{LpL}$ in other cells including myocytes, adipocytes, and endothelial cells ${ }^{53}$ distinctly affects lipid accumulation and the phenotypes of circulating monocytes and ATMs. Hypertriglyceridemia caused by global $L p /$ deficiency increases lipid accumulation in monocytes and shifts them to a more M2 phenotype. Global, but not M $\phi$-specific, $L p /$ deficiency in AT decreases lipid accumulation in ATMs and reduces FBC population without affecting M1/M2 polarity. Whether these findings were due to the global knockout causing changes in circulating TG-rich lipoproteins that made them reliant on $M \phi L p L$ or due to a requirement for adipose $L p L$ in the $M \phi$-lipid uptake process is unclear. Most likely, it suggests that multiple sources of lipids are responsible for creation of lipid-rich ATMs. Furthermore, our data support several recent and novel observations related to $L p L$ and cellular lipid 
metabolism. Most importantly, a LpL-rich cluster of cells has been found in the bone marrow using single cells RNA sequencing ${ }^{54}$. Thus, it is possible that $\mathrm{LpL}$ directs cellular phenotyping in ways that are exclusive of $M \phi$ lipid uptake. Within the AT, our data show that M $\phi L p L$ expression does not alter M $\phi$ lipid content and is not likely to be a major modulator of lipid uptake of the recently described adipose-derived exosomes ${ }^{47}$.

In conclusion, our current findings and the previous in vivo studies demonstrate that the dogma of M1/M2 dichotomy is only applicable in certain tissue culture conditions and clearly not in vivo physiology. The in vivo tissue microenvironment is far more complicated than culture conditions because it involves dynamic interactions between different cell types, energy sources, and many other factors.

\section{Acknowledgements}

HRC conducted all in vivo and in vitro experiments and analyzed the data presented in Figures 1-10. She primarily directed the project and wrote the manuscript. TJ performed the experiments and the data analyses for Figure 11, and she wrote the sections describing the methods related to those results. Both $\mathrm{HRC}$ and $\mathrm{TJ}$ revised the manuscript. DS, YH, LG, and TB assisted with the in vivo mouse studies. NG, SC, JG, and SB contributed to parts of the in vivo and in vitro experiments and performed qRTPCR and analyzed the data.

\section{Sources of Funding}

R01 HL135987, P01 HL092969, NYU Clinical \& Translational Science Awards Grant 1TL1 TR001447 from NIH, AHA Predoctoral Fellowship (18PRE33990436)

\section{Disclosures}

None 


\section{References}

1. Pollard JW. Trophic macrophages in development and disease. Nat Rev Immunol. 2009;9:259-270

2. Pearce EL, Pearce EJ. Metabolic pathways in immune cell activation and quiescence. Immunity. 2013;38:633-643

3. Ganeshan K, Chawla A. Metabolic regulation of immune responses. Annu Rev Immunol. 2014;32:609-634

4. Rahman K, Vengrenyuk Y, Ramsey SA, Vila NR, Girgis NM, Liu J, Gusarova V, Gromada J, Weinstock A, Moore KJ, Loke P, Fisher EA. Inflammatory ly6chi monocytes and their conversion to $\mathrm{m} 2$ macrophages drive atherosclerosis regression. J Clin Invest. 2017

5. Vats D, Mukundan L, Odegaard JI, Zhang L, Smith KL, Morel CR, Wagner RA, Greaves DR, Murray PJ, Chawla A. Oxidative metabolism and pgc-1beta attenuate macrophage-mediated inflammation. Cell Metab. 2006;4:13-24

6. Huang SC, Smith AM, Everts B, Colonna M, Pearce EL, Schilling JD, Pearce EJ. Metabolic reprogramming mediated by the mtorc2-irf4 signaling axis is essential for macrophage alternative activation. Immunity. 2016;45:817-830

7. Jha AK, Huang SC, Sergushichev A, Lampropoulou V, Ivanova Y, Loginicheva E, Chmielewski K, Stewart KM, Ashall J, Everts B, Pearce EJ, Driggers EM, Artyomov MN. Network integration of parallel metabolic and transcriptional data reveals metabolic modules that regulate macrophage polarization. Immunity. 2015;42:419-430

8. Trent CM, Yu S, Hu Y, Skoller N, Huggins LA, Homma S, Goldberg IJ. Lipoprotein lipase activity is required for cardiac lipid droplet production. J Lipid Res. 2014;55:645-658

9. Garcia-Arcos I, Hiyama Y, Drosatos K, Bharadwaj KG, Hu Y, Son NH, O'Byrne SM, Chang CL, Deckelbaum RJ, Takahashi M, Westerterp M, Obunike JC, Jiang H, Yagyu H, Blaner WS, Goldberg IJ. Adipose-specific lipoprotein lipase deficiency more profoundly affects brown than white fat biology. J Biol Chem. 2013;288:1404614058

10. Augustus A, Yagyu H, Haemmerle G, Bensadoun A, Vikramadithyan RK, Park SY, Kim JK, Zechner R, Goldberg IJ. Cardiac-specific knock-out of lipoprotein lipase alters plasma lipoprotein triglyceride metabolism and cardiac gene expression. $J$ Biol Chem. 2004;279:25050-25057

11. Obunike JC, Edwards IJ, Rumsey SC, Curtiss LK, Wagner WD, Deckelbaum RJ, Goldberg IJ. Cellular differences in lipoprotein lipase-mediated uptake of low density lipoproteins. J Biol Chem. 1994;269:13129-13135

12. den Hartigh LJ, Connolly-Rohrbach JE, Fore S, Huser TR, Rutledge JC. Fatty acids from very low-density lipoprotein lipolysis products induce lipid droplet accumulation in human monocytes. J Immunol. 2010;184:3927-3936

13. Saraswathi V, Hasty AH. The role of lipolysis in mediating the proinflammatory effects of very low density lipoproteins in mouse peritoneal macrophages. J Lipid Res. 2006;47:1406-1415

14. Chawla A, Lee CH, Barak Y, He W, Rosenfeld J, Liao D, Han J, Kang H, Evans RM. Ppardelta is a very low-density lipoprotein sensor in macrophages. Proc Natl Acad Sci U S A. 2003;100:1268-1273

15. Wu C, Jin X, Tsueng G, Afrasiabi C, Su AI. Biogps: Building your own mash-up of gene annotations and expression profiles. Nucleic Acids Res. 2016;44:D313-316 
16. Yin B, Loike JD, Kako Y, Weinstock PH, Breslow JL, Silverstein SC, Goldberg IJ. Lipoprotein lipase regulates fc receptor-mediated phagocytosis by macrophages maintained in glucose-deficient medium. J Clin Invest. 1997;100:649-657

17. den Hartigh LJ, Altman R, Norman JE, Rutledge JC. Postprandial vldl lipolysis products increase monocyte adhesion and lipid droplet formation via activation of erk2 and nfkappab. Am J Physiol Heart Circ Physiol. 2014;306:H109-120

18. Bojic LA, Sawyez CG, Telford DE, Edwards JY, Hegele RA, Huff MW. Activation of peroxisome proliferator-activated receptor delta inhibits human macrophage foam cell formation and the inflammatory response induced by very low-density lipoprotein. Arterioscler Thromb Vasc Biol. 2012;32:2919-2928

19. Lee CH, Kang K, Mehl IR, Nofsinger R, Alaynick WA, Chong LW, Rosenfeld JM, Evans RM. Peroxisome proliferator-activated receptor delta promotes very lowdensity lipoprotein-derived fatty acid catabolism in the macrophage. Proc Natl Acad Sci U S A. 2006;103:2434-2439

20. Barish GD, Atkins AR, Downes M, Olson P, Chong LW, Nelson M, Zou Y, Hwang H, Kang H, Curtiss L, Evans RM, Lee CH. Ppardelta regulates multiple proinflammatory pathways to suppress atherosclerosis. Proc Natl Acad Sci U S A. 2008;105:4271-4276

21. Takahashi M, Yagyu H, Tazoe F, Nagashima S, Ohshiro T, Okada K, Osuga J, Goldberg IJ, Ishibashi S. Macrophage lipoprotein lipase modulates the development of atherosclerosis but not adiposity. J Lipid Res. 2013;54:1124-1134

22. Bharadwaj KG, Hiyama Y, Hu Y, Huggins LA, Ramakrishnan R, Abumrad NA, Shulman GI, Blaner WS, Goldberg IJ. Chylomicron- and vldl-derived lipids enter the heart through different pathways: In vivo evidence for receptor- and nonreceptor-mediated fatty acid uptake. J Biol Chem. 2010;285:37976-37986

23. Nagareddy PR, Murphy AJ, Stirzaker RA, Hu Y, Yu S, Miller RG, Ramkhelawon B, Distel E, Westerterp M, Huang LS, Schmidt AM, Orchard TJ, Fisher EA, Tall AR, Goldberg IJ. Hyperglycemia promotes myelopoiesis and impairs the resolution of atherosclerosis. Cell Metab. 2013;17:695-708

24. Xu X, Grijalva A, Skowronski A, van Eijk M, Serlie MJ, Ferrante AW, Jr. Obesity activates a program of lysosomal-dependent lipid metabolism in adipose tissue macrophages independently of classic activation. Cell Metab. 2013;18:816-830

25. Okabe Y, Medzhitov R. Tissue-specific signals control reversible program of localization and functional polarization of macrophages. Cell. 2014;157:832-844

26. Trogan E, Choudhury RP, Dansky HM, Rong JX, Breslow JL, Fisher EA. Laser capture microdissection analysis of gene expression in macrophages from atherosclerotic lesions of apolipoprotein e-deficient mice. Proc Natl Acad Sci U S A. 2002;99:2234-2239

27. Trogan E, Fayad ZA, Itskovich VV, Aguinaldo JG, Mani V, Fallon JT, Chereshnev I, Fisher EA. Serial studies of mouse atherosclerosis by in vivo magnetic resonance imaging detect lesion regression after correction of dyslipidemia. Arterioscler Thromb Vasc Biol. 2004;24:1714-1719

28. Reis ED, Li J, Fayad ZA, Rong JX, Hansoty D, Aguinaldo JG, Fallon JT, Fisher EA. Dramatic remodeling of advanced atherosclerotic plaques of the apolipoprotein e-deficient mouse in a novel transplantation model. J Vasc Surg. 2001;34:541-547

29. Chereshnev I, Trogan E, Omerhodzic S, Itskovich V, Aguinaldo JG, Fayad ZA, Fisher EA, Reis ED. Mouse model of heterotopic aortic arch transplantation. J Surg Res. 2003;111:171-176 
30. Daugherty A, Tall AR, Daemen M, Falk E, Fisher EA, Garcia-Cardena G, Lusis AJ, Owens AP, 3rd, Rosenfeld ME, Virmani R, American Heart Association Council on Arteriosclerosis T, Vascular B, Council on Basic Cardiovascular S.

Recommendation on design, execution, and reporting of animal atherosclerosis studies: A scientific statement from the american heart association. Arterioscler Thromb Vasc Biol. 2017;37:e131-e157

31. Nunnari JJ, Zand T, Joris I, Majno G. Quantitation of oil red o staining of the aorta in hypercholesterolemic rats. Exp Mol Pathol. 1989;51:1-8

32. Feng B, Zhang D, Kuriakose G, Devlin CM, Kockx M, Tabas I. Niemann-pick c heterozygosity confers resistance to lesional necrosis and macrophage apoptosis in murine atherosclerosis. Proc Natl Acad Sci U S A. 2003;100:10423-10428

33. Camell C, Smith CW. Dietary oleic acid increases $\mathrm{m} 2$ macrophages in the mesenteric adipose tissue. PloS one. 2013;8:e75147

34. Pardo V, Gonzalez-Rodriguez A, Guijas C, Balsinde J, Valverde AM. Opposite crosstalk by oleate and palmitate on insulin signaling in hepatocytes through macrophage activation. J Biol Chem. 2015;290:11663-11677

35. Odegaard JI, Ricardo-Gonzalez RR, Red Eagle A, Vats D, Morel CR, Goforth MH, Subramanian V, Mukundan L, Ferrante AW, Chawla A. Alternative $\mathrm{m} 2$ activation of kupffer cells by ppardelta ameliorates obesity-induced insulin resistance. Cell Metab. 2008;7:496-507

36. Nishizawa T, Kanter JE, Kramer F, Barnhart S, Shen X, Vivekanandan-Giri A, Wall VZ, Kowitz J, Devaraj S, O'Brien KD, Pennathur S, Tang J, Miyaoka RS, Raines EW, Bornfeldt KE. Testing the role of myeloid cell glucose flux in inflammation and atherosclerosis. Cell Rep. 2014;7:356-365

37. Nagareddy PR, Kraakman M, Masters SL, Stirzaker RA, Gorman DJ, Grant RW, Dragoljevic D, Hong ES, Abdel-Latif A, Smyth SS, Choi SH, Korner J, Bornfeldt KE, Fisher EA, Dixit VD, Tall AR, Goldberg IJ, Murphy AJ. Adipose tissue macrophages promote myelopoiesis and monocytosis in obesity. Cell Metab. 2014;19:821-835

38. Chang CL, Garcia-Arcos I, Nyren R, Olivecrona G, Kim JY, Hu Y, Agrawal RR, Murphy AJ, Goldberg IJ, Deckelbaum RJ. Lipoprotein lipase deficiency impairs bone marrow myelopoiesis and reduces circulating monocyte levels. Arterioscler Thromb Vasc Biol. 2018;38:509-519

39. Aouadi M, Vangala P, Yawe JC, Tencerova M, Nicoloro SM, Cohen JL, Shen Y, Czech MP. Lipid storage by adipose tissue macrophages regulates systemic glucose tolerance. Am J Physiol Endocrinol Metab. 2014;307:E374-383

40. Lumeng CN, Bodzin JL, Saltiel AR. Obesity induces a phenotypic switch in adipose tissue macrophage polarization. J Clin Invest. 2007;117:175-184

41. Muoio DM, MacLean PS, Lang DB, Li S, Houmard JA, Way JM, Winegar DA, Corton JC, Dohm GL, Kraus WE. Fatty acid homeostasis and induction of lipid regulatory genes in skeletal muscles of peroxisome proliferator-activated receptor (ppar) alpha knock-out mice. Evidence for compensatory regulation by ppar delta. J Biol Chem. 2002;277:26089-26097

42. Gordts PL, Nock R, Son NH, Ramms B, Lew I, Gonzales JC, Thacker BE, Basu D, Lee RG, Mullick AE, Graham MJ, Goldberg IJ, Crooke RM, Witztum JL, Esko JD. Apoc-iii inhibits clearance of triglyceride-rich lipoproteins through ld family receptors. J Clin Invest. 2016;126:2855-2866

43. Merkel M, Weinstock PH, Chajek-Shaul T, Radner H, Yin B, Breslow JL, Goldberg IJ. Lipoprotein lipase expression exclusively in liver. A mouse model for 
metabolism in the neonatal period and during cachexia. J Clin Invest. 1998;102:893-901

44. Lichtenstein L, Mattijssen F, de Wit NJ, Georgiadi A, Hooiveld GJ, van der Meer R, He Y, Qi L, Koster A, Tamsma JT, Tan NS, Muller M, Kersten S. Angptl4 protects against severe proinflammatory effects of saturated fat by inhibiting fatty acid uptake into mesenteric lymph node macrophages. Cell Metab. 2010;12:580-592

45. Feig JE, Fisher EA. Laser capture microdissection for analysis of macrophage gene expression from atherosclerotic lesions. Methods Mol Biol. 2013;1027:123-135

46. Gordon S, Martinez FO. Alternative activation of macrophages: Mechanism and functions. Immunity. 2010;32:593-604

47. Flaherty SE, 3rd, Grijalva A, Xu X, Ables E, Nomani A, Ferrante AW, Jr. A lipaseindependent pathway of lipid release and immune modulation by adipocytes. Science. 2019;363:989-993

48. Nascimento M, Huang SC, Smith A, Everts B, Lam W, Bassity E, Gautier EL, Randolph GJ, Pearce EJ. Ly6chi monocyte recruitment is responsible for th2 associated host-protective macrophage accumulation in liver inflammation due to schistosomiasis. PLoS Pathog. 2014;10:e1004282

49. Gonzalez-Hurtado E, Lee J, Choi J, Selen Alpergin ES, Collins SL, Horton MR, Wolfgang MJ. Loss of macrophage fatty acid oxidation does not potentiate systemic metabolic dysfunction. Am J Physiol Endocrinol Metab. 2017;312:E381E393

50. Kitayama J, Emoto S, Yamaguchi H, Ishigami H, Watanabe T. Cd90+ mesotheliallike cells in peritoneal fluid promote peritoneal metastasis by forming a tumor permissive microenvironment. PloS one. 2014;9:e86516

51. Ghosn EE, Cassado AA, Govoni GR, Fukuhara T, Yang Y, Monack DM, Bortoluci KR, Almeida SR, Herzenberg LA, Herzenberg LA. Two physically, functionally, and developmentally distinct peritoneal macrophage subsets. Proc Natl Acad Sci U S A. 2010;107:2568-2573

52. Chinetti-Gbaguidi G, Colin S, Staels B. Macrophage subsets in atherosclerosis. Nat Rev Cardiol. 2015;12:10-17

53. Goldberg IJ, Eckel RH, Abumrad NA. Regulation of fatty acid uptake into tissues: Lipoprotein lipase- and cd36-mediated pathways. J Lipid Res. 2009;50 Suppl:S8690

54. Tikhonova AN, Dolgalev I, Hu H, Sivaraj KK, Hoxha E, Cuesta-Dominguez A, Pinho S, Akhmetzyanova I, Gao J, Witkowski M, Guillamot M, Gutkin MC, Zhang Y, Marier C, Diefenbach C, Kousteni S, Heguy A, Zhong H, Fooksman DR, Butler JM, Economides A, Frenette PS, Adams RH, Satija R, Tsirigos A, Aifantis I. The bone marrow microenvironment at single-cell resolution. Nature. 2019;569:222-228 


\section{Supplemental Methods}

Animals: All procedures were approved by the Institutional Animal Care and Use Committee at New York University Langone Health. Mice were maintained in a temperature-controlled $\left(25^{\circ} \mathrm{C}\right)$ facility with a $12 \mathrm{~h}$-light/dark cycle and given free access to water and food, except when fasting blood was obtained. Mice were fed a laboratory standard rodent chow diet or a high fat diet (HFD) (60\% fat kcal; Research Diet \#D12492), as indicated. Euthanasia was done by intraperitoneal injection of ketamine $100 \mathrm{mg} / \mathrm{kg}$ and xylazine $10 \mathrm{mg} / \mathrm{kg}$ followed by cervical dislocation.

Blood Leukocytes: Tail blood was collected into EDTA-lined tubes and immediately placed on ice. All subsequent steps were performed on ice. Red blood cells were lysed, and white blood cells were centrifuged, washed, and resuspended in FACS buffer. Cells were stained with a cocktail of antibodies against CD45-Alexa Fluor 450 (Invitrogen), Ly6-C/G-PerCP-Cy5.5 (BD Pharmigen), and CD115- APC (eBioscience). Monocytes were identified as CD45hiCD115hi and further identified into Ly6Chi and Ly6C ${ }^{\text {lo; }}$, neutrophils were identified as CD45hiCD115loLy6-C/Ghi (Gr-1).

Hematopoietic Stem Cells: BM was harvested from femurs and tibiae by flushing with ice cold PBS. Spleens were minced into small pieces, gently rubbed against the mesh in a cell strainer and flushed with PBS. The cell suspension was centrifuged at $500 \mathrm{~g}$ for 5 mins. The supernatant was aspirated and the cells were resuspended in red blood cell lysis buffer for 5 mins. Lysis was stopped by adding an excess amount of flow buffer, and the cell suspension was once again centrifuged and aspirated. A single cell suspension was prepared by gently pipetting up and down with $200 \mu \mathrm{l}$ of flow buffer. This was followed by incubation with a cocktail of antibodies against lineage-committed cells (B220, CD19, CD11b, CD3e, TER-119, CD2, CD8, CD4, Ly6-C/G: All FITC, eBioscience), Sca1-Pacific Blue, and ckit-APC Cy7). HSPCs were identified as lin-, Sac1+, and ckit+ (LSK), while the hematopoietic progenitor subsets were separated using antibodies to CD16/CD32 (FcyRII/III) and CD34. Common myeloid progenitors were identified as lin-, Sca1-, ckit+, CD34int, FcyRII/Illint, and granulocyte-M $\phi$ progenitors as lin-, Sca1-, ckit+, CD34int, FcyRII/IIlhi. BM M $\phi$ s were identified by negative gating against (B220, CD19, CD11b, CD3e, TER-119, CD2, CD8, CD4, Ly6 C/G: All FITC, eBioscience) and then CD115+, Gr1lo, F4/80+, CD169+, and either CD11c positive or negative. Flow cytometry was performed using an LSRII (for analysis) or FACS Aria (for sorting), with both machines running FACS DiVa software. 
All flow cytometry data were analyzed using FlowJo software (Tree Star Inc.).

Adipose Tissue Macrophage (ATM) Isolation: Freshly dissected adipose tissue was minced into fine pieces ( $<10 \mathrm{mg}$ ) and centrifuged to remove connective tissue debris. Minced tissues were digested by liberase TM (Roche) for 20 mins and centrifuged at $500 \mathrm{~g}$ for 5 mins. The pellets were collected as stromal vascular cells (SVCs), and buoyant cells were collected as adipocytes. The SVCs were resuspended in erythrocyte lysis buffer (BD Biosciences) and the erythrocyte-depleted SVCs were incubated with F4/80-APC, CD11b-PE, and CD11c-PETR for M $\phi$ purification. ATM subpopulations were separated by $F 4 / 80+C D 11 b+(F B s)$ and $F 4 / 80+C D 11 b+C D 11 c+$ (FBCs); the gating strategy is shown in Supplemental Figure $\mathrm{V}$.

Quantitative Real Time-PCR Analyses: Total RNA was extracted from cells with silica-based membrane method by using PureLink RNA Mini kit (Ambion) for BMDMs or RNeasy Micro kit (Qiagen) for FACS-sorted cells. Complementary DNAs (cDNAs) were generated with a Verso cDNA synthesis kit (Thermo Fisher), and quantitative PCR was carried out with fluorescent double-stranded DNA dye (Fast SYBR Green Mastermix; Applied Biosystems). Data were normalized to Rps3 with the $\Delta \Delta \mathrm{C}(\mathrm{t})$ method and are presented as relative transcript levels. All primers are listed in Supplemental Table I. 


\section{Supplemental Figures}

Supplemental Table I. List of primers used for quantitative PCR

\begin{tabular}{|c|c|c|}
\hline Gene & Forward (5' - 3') & Reverse (5' - 3') \\
\hline Rps3 & ATCAGAGAGTTGACCGCAGTTG & AATGAACCGAAGCACACCATAG \\
\hline Lpl & AGGTGGACATCGGAGAACTG & TCCCTAGCACAGAAGATGACC3 \\
\hline Plin2 & TCTGCGGCCATGACAAGTG & GCAGGCATAGGTATTGGCAAC \\
\hline Cpt1a & TGCACTACGGAGTCCTGCAA & GGACAACCTCCATGGCTCAG \\
\hline Glut1 & TCGTAACGAGGAGAACCG & GGCCGTGTTGACGATA \\
\hline Fasn & TCTTTCTAACAACCACCСTCTGG & CTTCACGACTCCATCACGAATG \\
\hline Cd36 & CCTTAAAGGAATCCCCGTGT & TGCATTTGCCAATGTCTAGC \\
\hline Tnf & TGGAACTGGCAGAAGAGG & AGACAGAAGAGCGTGGTG \\
\hline II1b & CAACCAACAAGTGATATTCTCCATG & GATCCACACTCTCCAGCTGCA \\
\hline Mcp1 & CCCAATGAGTAGGCTGGAGA & TCTGGACCCATTCCTTCTTG \\
\hline Nos2 & CAGCTGGGCTGTACAAACCTT & CATTGGAAGTGAAGCGTTTCG \\
\hline $\operatorname{Arg} 1$ & TGGCTTGCGAGACGTAGAC & GCTCAGGTGAATCGGCCTTTT \\
\hline$Y m 1$ & AGGCTTTGCGGTCCTGAT & CCAGCTGGTGAAGTAGCAGA \\
\hline Fizz1 & CCAATCCAGCTAACTATCCCTCC & AAGCCACAAGCACACCCAGT \\
\hline Mrc1 & TGATTACGAGCAGTGGAAGC & GTTCACCGTA-AGCCCAATTT \\
\hline II10 & CTGGACAACATACTGCTAACCG & GGGCATCACTTCTACCAGGTAA \\
\hline
\end{tabular}


A

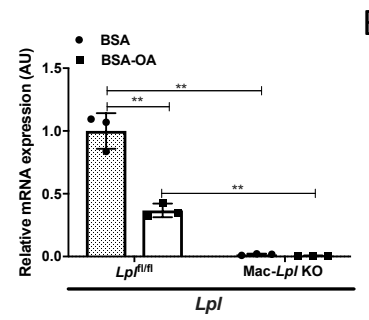

D
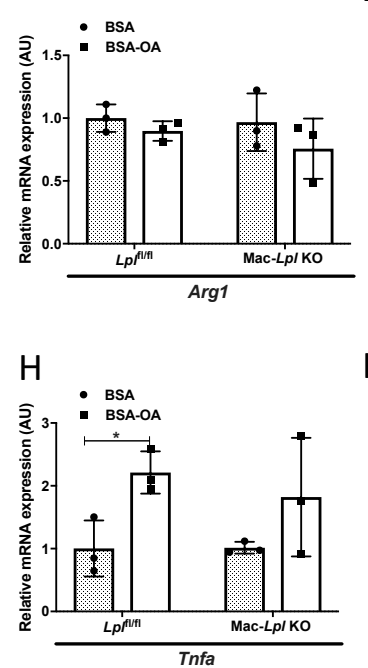

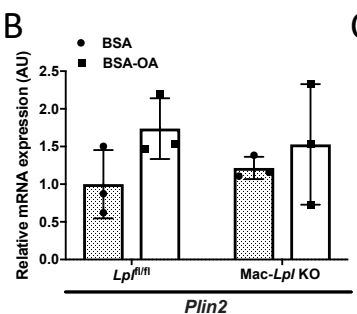

$E$
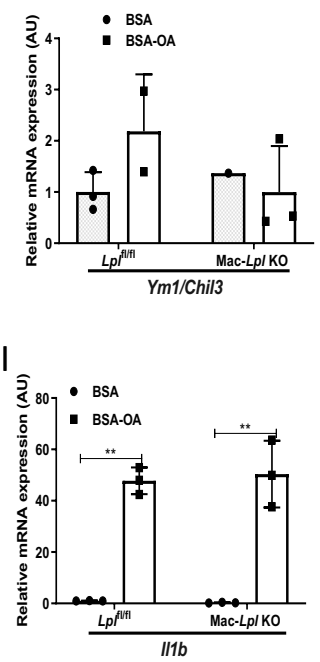

C

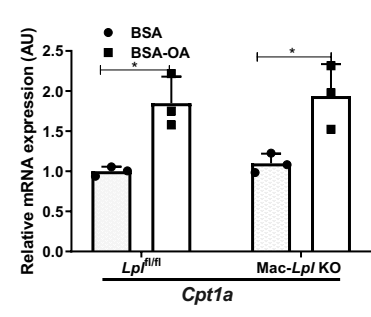

G
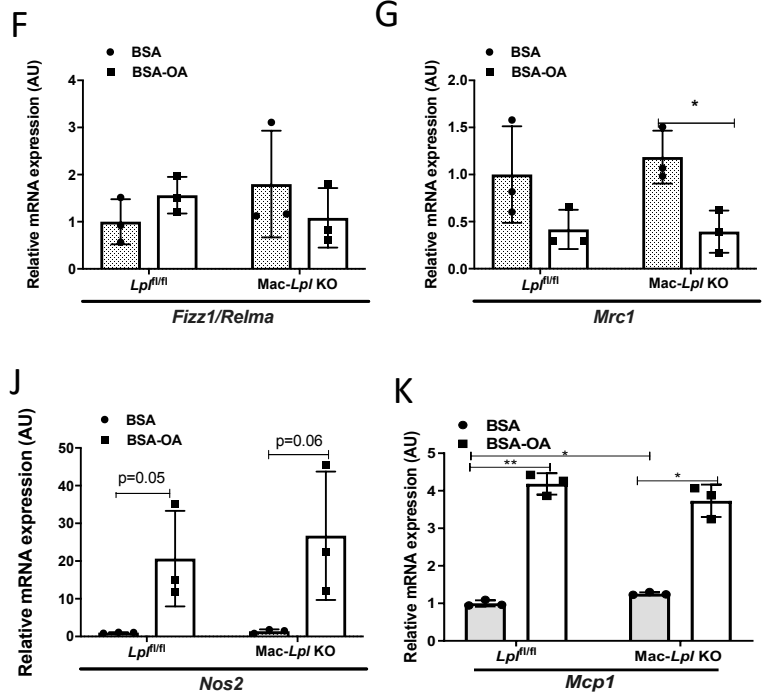

K

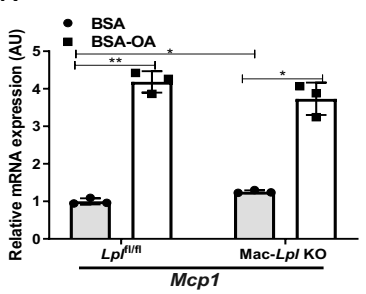

\section{Supplemental Figure I. Lpl deficiency does not affect alternative activation of} macrophages upon oleic acid treatment in vitro

BMDMs from $L p^{f / f l l}$ and Mac- $L p H^{-/}$mice were used to measure metabolism- related and M1/M2-related gene expression in the presence of oleic acid (OA). (A) The gene expression of $L p /$ in BMDMs. (B) The expression of a lipid droplet-related protein, Plin2, gene in BMDMs. (C) The expression of a fatty acid oxidation-related gene, Cpt1a in BMDMs. (D-G) gene expression of anti-inflammatory genes (Arg1, Ym1, Fizz1, Mrc1) in OA treated BMDMs. (H-K) The expression of inflammatory genes (Tnfa, 11b, Nos2, Mcp1) in OA treated BMDMs. $N=3$ /group. ${ }^{*} p<0.05,{ }^{* *} p<0.01$. Results are represented mean $\pm S D$ and compared using Two-way ANOVA, Sidak's multiple comparison. 

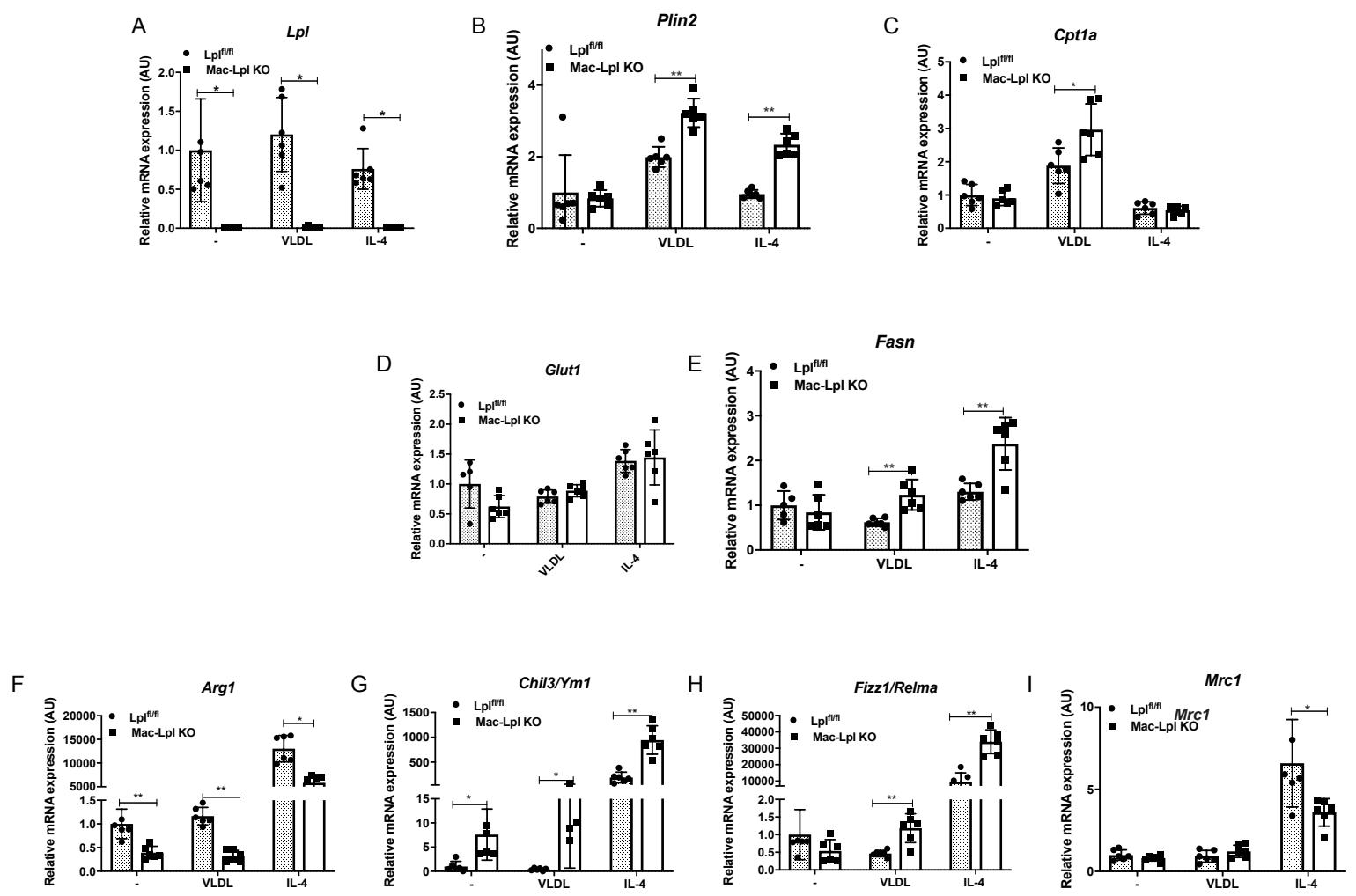

\section{Supplemental Figure II. BMDMs culture in normal glucose $(5 \mathrm{mM})$}

BMDMs from $L p f^{f / f l}$ and Mac- $L p F^{-/}$mice were used to measure metabolism- related and M1/M2-related gene expression in the presence of human VLDL $(100 \mathrm{ug} / \mathrm{mL})$, IL-4 $(20 \mathrm{ng} / \mathrm{mL})$ and normal glucose (5mM). (A) The gene expression of $L p /$ in BMDMs. (B) The expression of a lipid droplet-related protein, Plin2, gene in BMDMs. (C) The expression of a fatty acid oxidation-related gene, Cpt1a in BMDMs. (D) The expression of a glucose transporter, Glut1 (S/c2a1), gene in BMDMs. (E)The expression of fatty acid synthase gene in BMDMs. (F-I) The expression of canonical M2 (anti-inflammatory) genes in BMDMs. $N=6$ /group. ${ }^{*} p<0.05,{ }^{* *} p<0.01$. Results are represented mean $\pm S D$ and compared using Twoway ANOVA, Sidak's multiple comparison. 

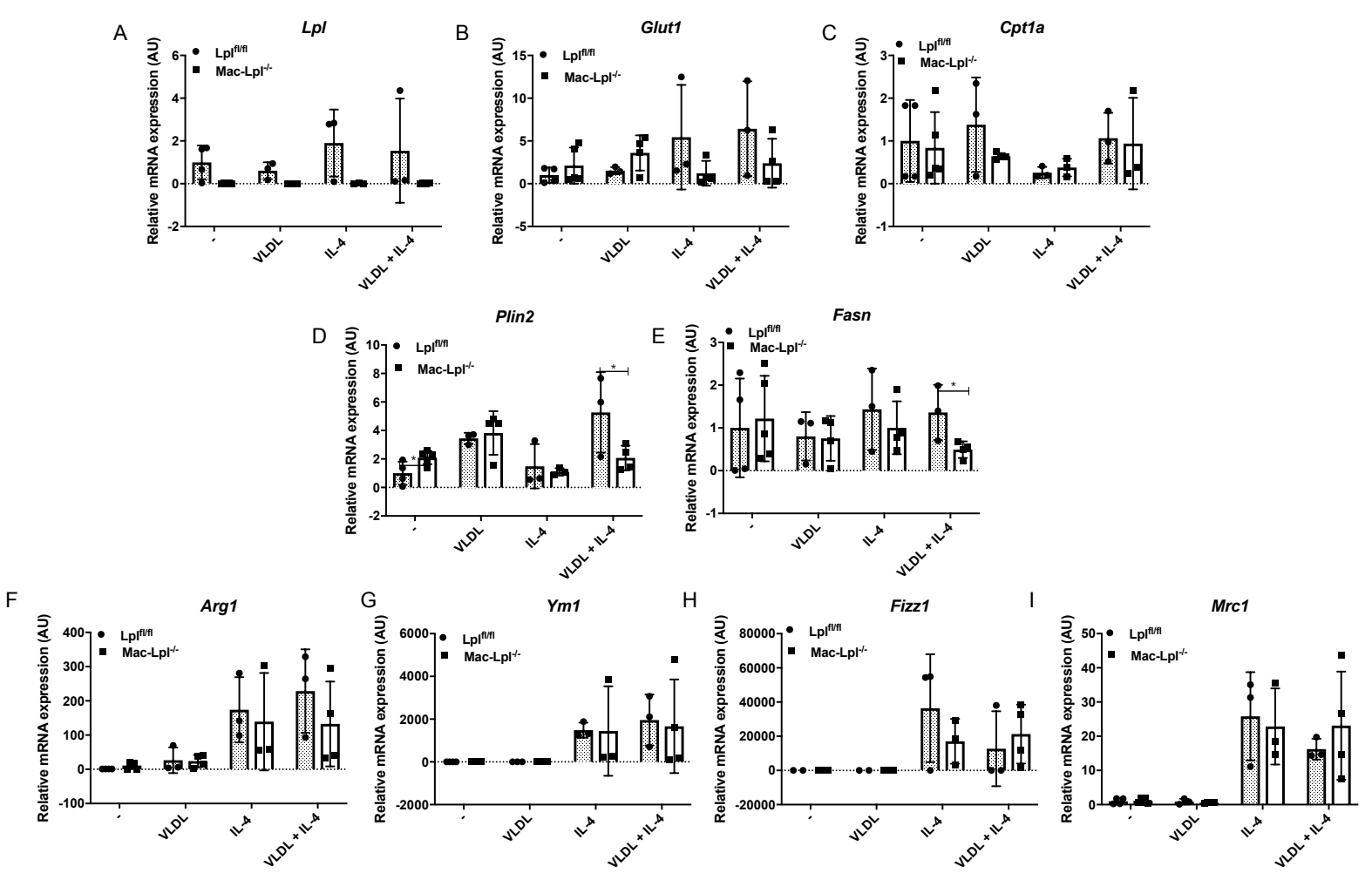

\section{Supplemental Figure III. BMDMs cultured in high $(25 \mathrm{mM})$ glucose}

BMDMs from $L p f^{f / f l}$ and Mac- $L p F^{-1-}$ mice were used to measure metabolism- related and M1/M2-related gene expression in the presence of human VLDL (100ug/mL), IL-4 $(20 \mathrm{ng} / \mathrm{mL})$ and high glucose $(25 \mathrm{mM})$. (A) The gene expression of $L p /$ in BMDMs. (B) The expression of a glucose transporter, Glut1 (SIc2a1), gene in BMDMs. (C) The expression of a fatty acid oxidation-related gene, Cpt1a in BMDMs. (D) The expression of a lipid droplet-related protein, Plin2, gene in BMDMs. (E)The expression of fatty acid synthase gene in BMDMs. (F-I) The expression of canonical M2 (anti-inflammatory) genes in BMDMs. $\mathrm{N}=3$-4/group. ${ }^{*} \mathrm{p}<0.05,{ }^{* *} \mathrm{p}<0.01$. Results are represented mean $\pm \mathrm{SD}$ and compared using Two-way ANOVA, Sidak's multiple comparison. 


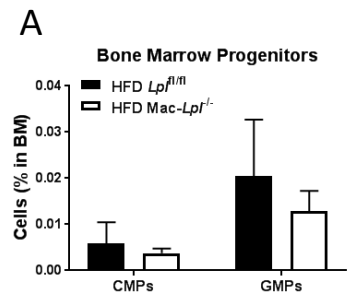

D

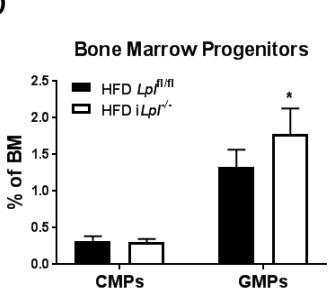

B

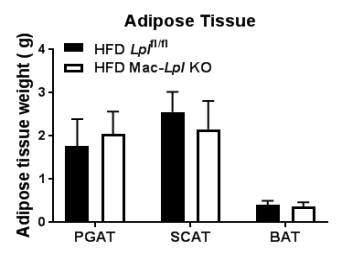

$\mathrm{F}$

C
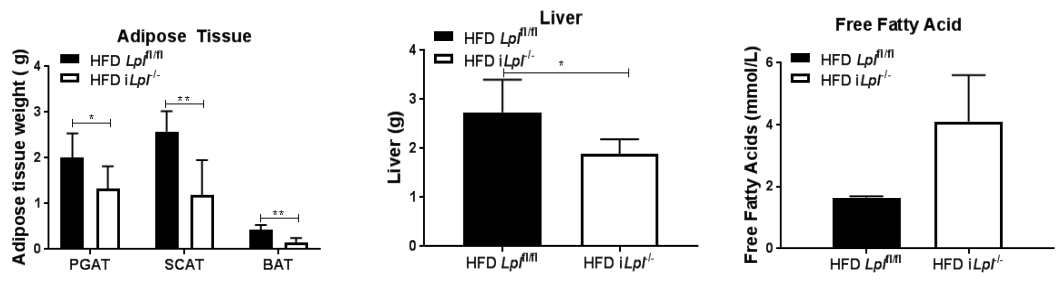

Supplemental Figure IV. Bone marrow progenitors and weight of tissue in Mac $L p I^{-/-a n d}$ i $L p I^{---}$mice fed a HFD

(A) Bone marrow progenitors in Mac- $\mathrm{LpH}^{--}$(B) Adipose tissue weight (g) in Mac- $\mathrm{LpF}^{--}$(C) Liver weight (g)

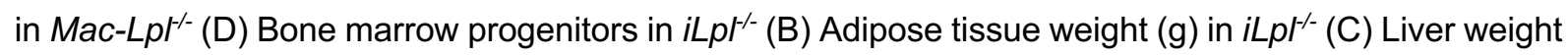

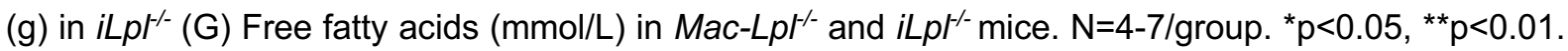
Results are represented mean \pm SD and compared using Two-way ANOVA, Sidak's multiple comparison. 


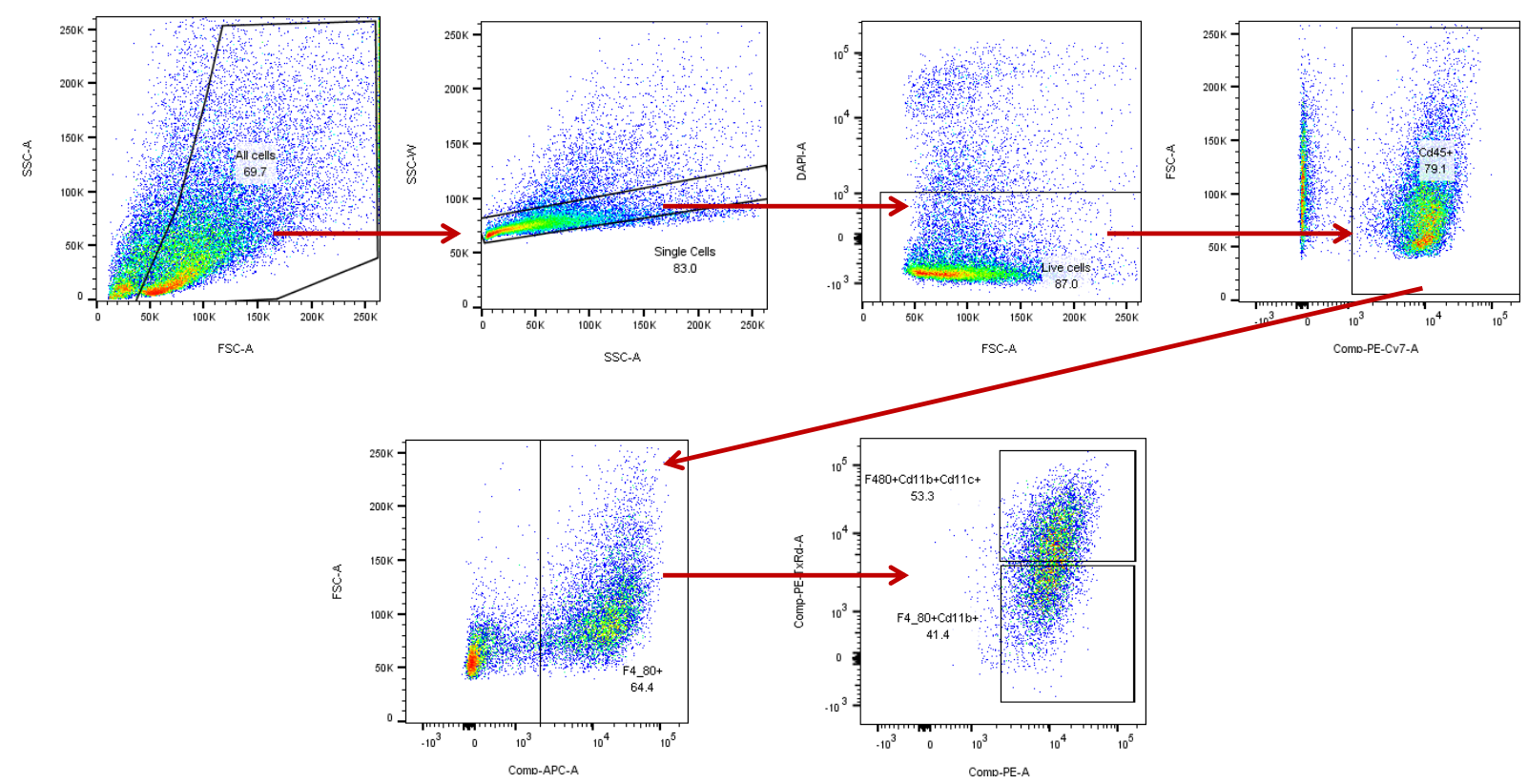

Supplemental Figure V. Adipose tissue macrophages (ATM) gating strategy 
Chapter 3

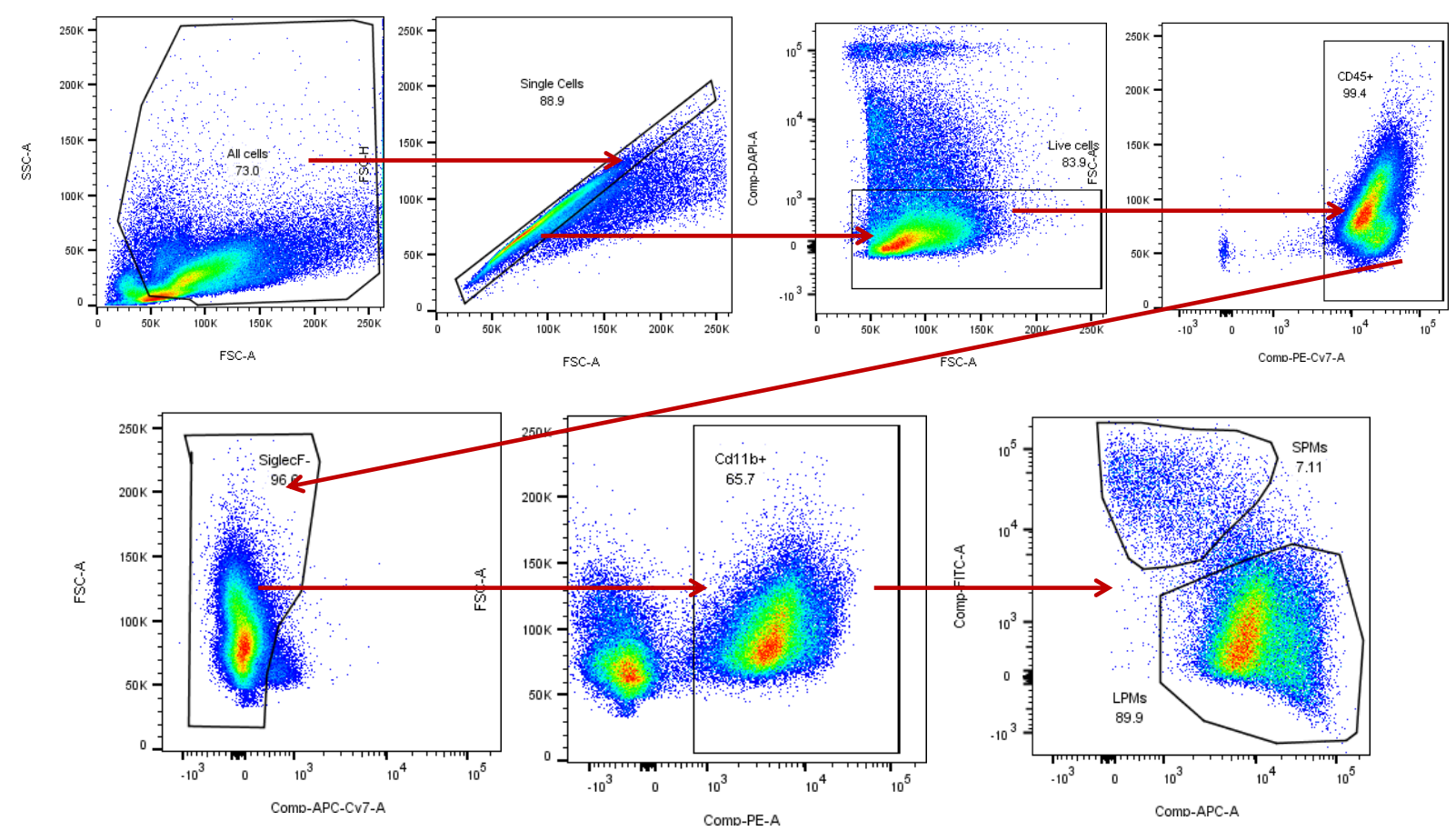

Supplemental Figure VI. Peritoneal macrophages gating strategy 

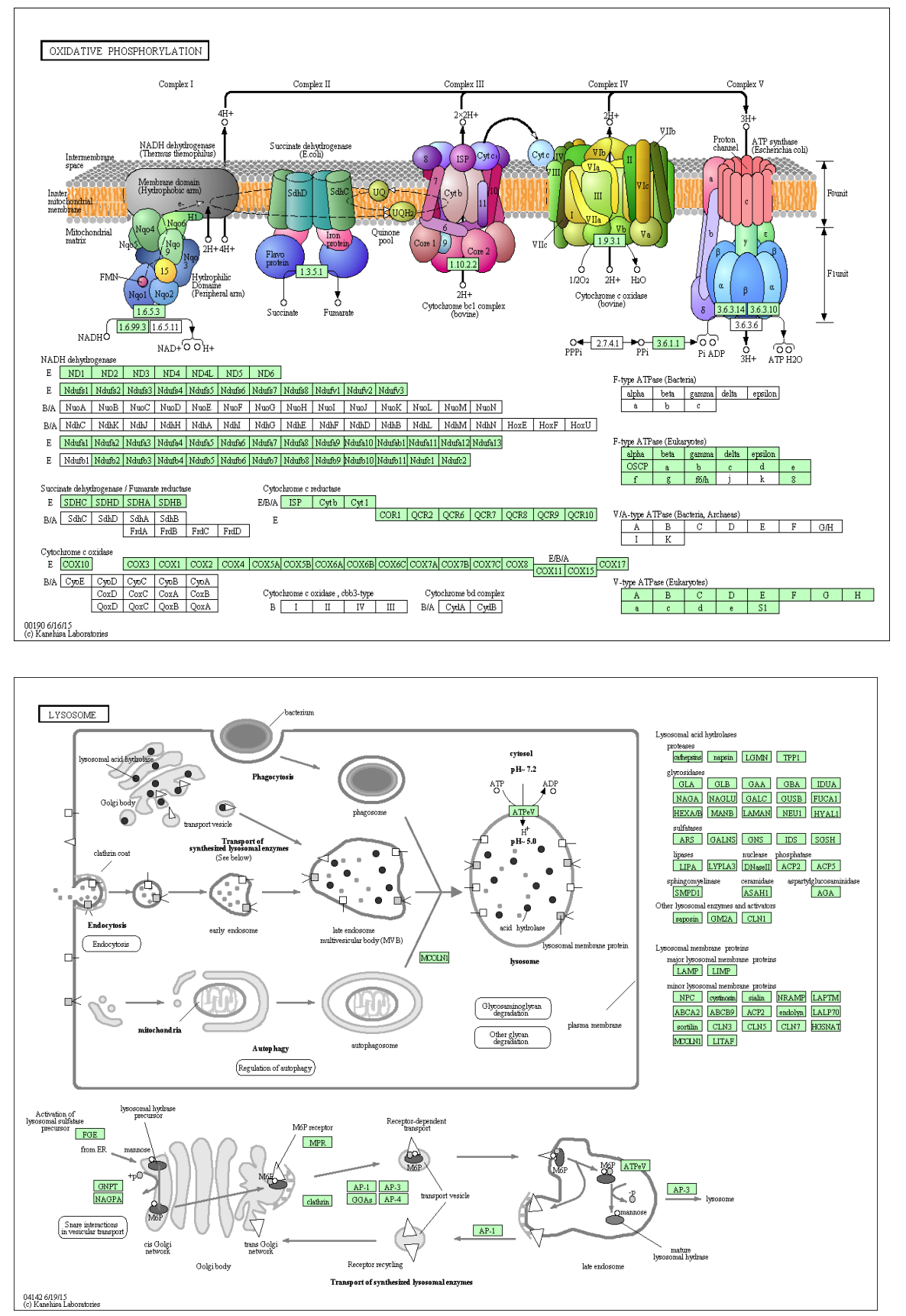

Supplemental Figure VII. KEGG Mapper Analysis- Oxidative Phosphorylation and Lysosome Pathway (Genes cut off q-value <0.05) 
Chapter 3

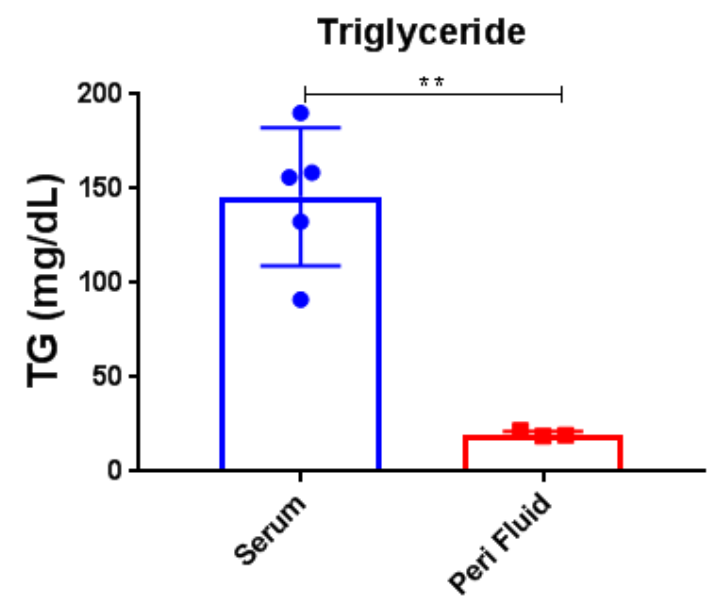

Supplemental Figure VIII. TG levels in plasma versus TG levels in peritoneal fluid 
A

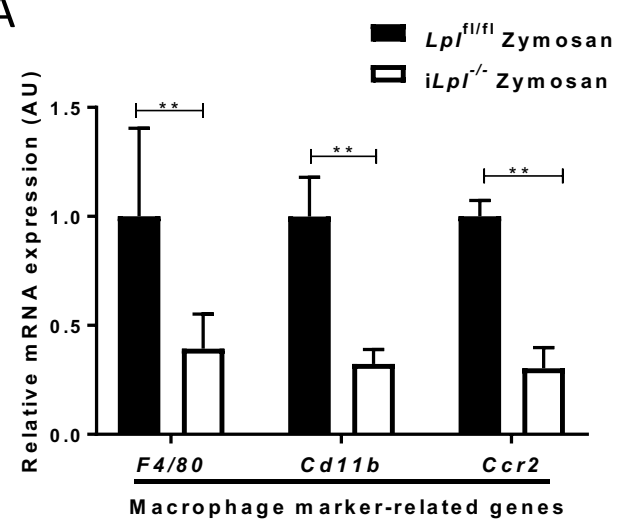

C

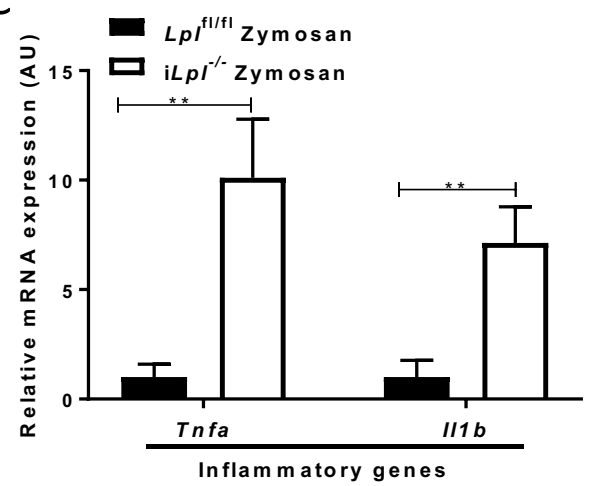

B

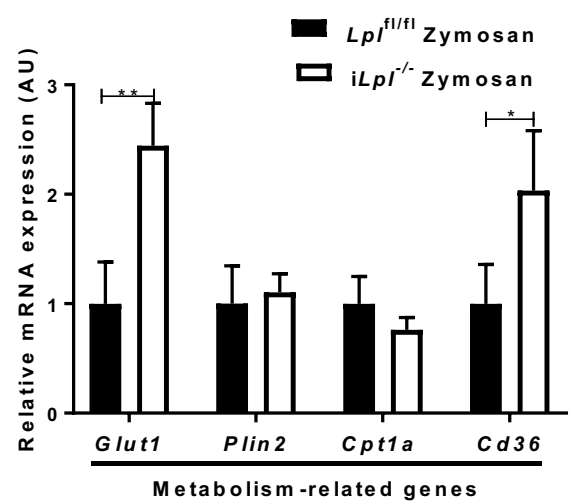

D

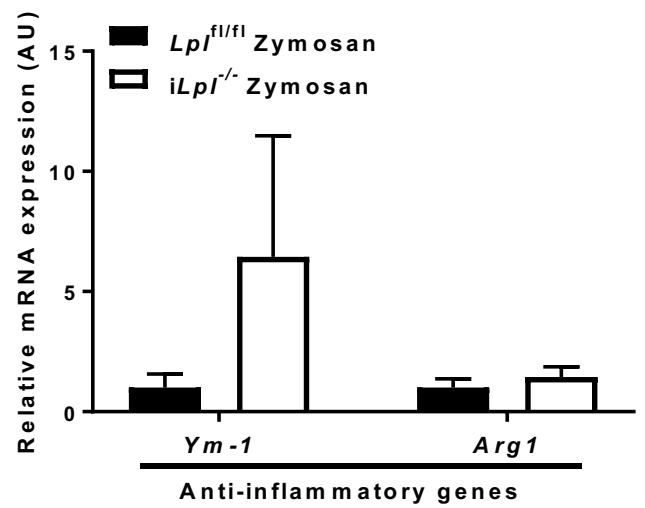

Supplemental Figure IX. Inflammatory and anti-inflammatory gene expression in total peritoneal macrophages upon zymosan stimuli

Peritoneal Macrophages from $L p f^{f / f l}$ and $i L p r^{--}$mice were used to measure metabolism- related and M1/M2-related gene expression. (A) Expression of macrophage marker related genes (F4/80, Cd11b, Ccr2). (B) The expression of metabolism-related genes (Glut1, Plin2, Cpt1a, Cd36). (C) The expression of inflammatory genes (Tnfa, II1b).(D) The expression of anti-inflammatory genes (Ym-1, Arg1). $\mathrm{N}=4 /$ group, ${ }^{*} p<0.05,{ }^{* *} \mathrm{p}<0.01$. Results are represented mean $\pm \mathrm{SD}$ and compared using Two-way ANOVA, Sidak's multiple comparison. 

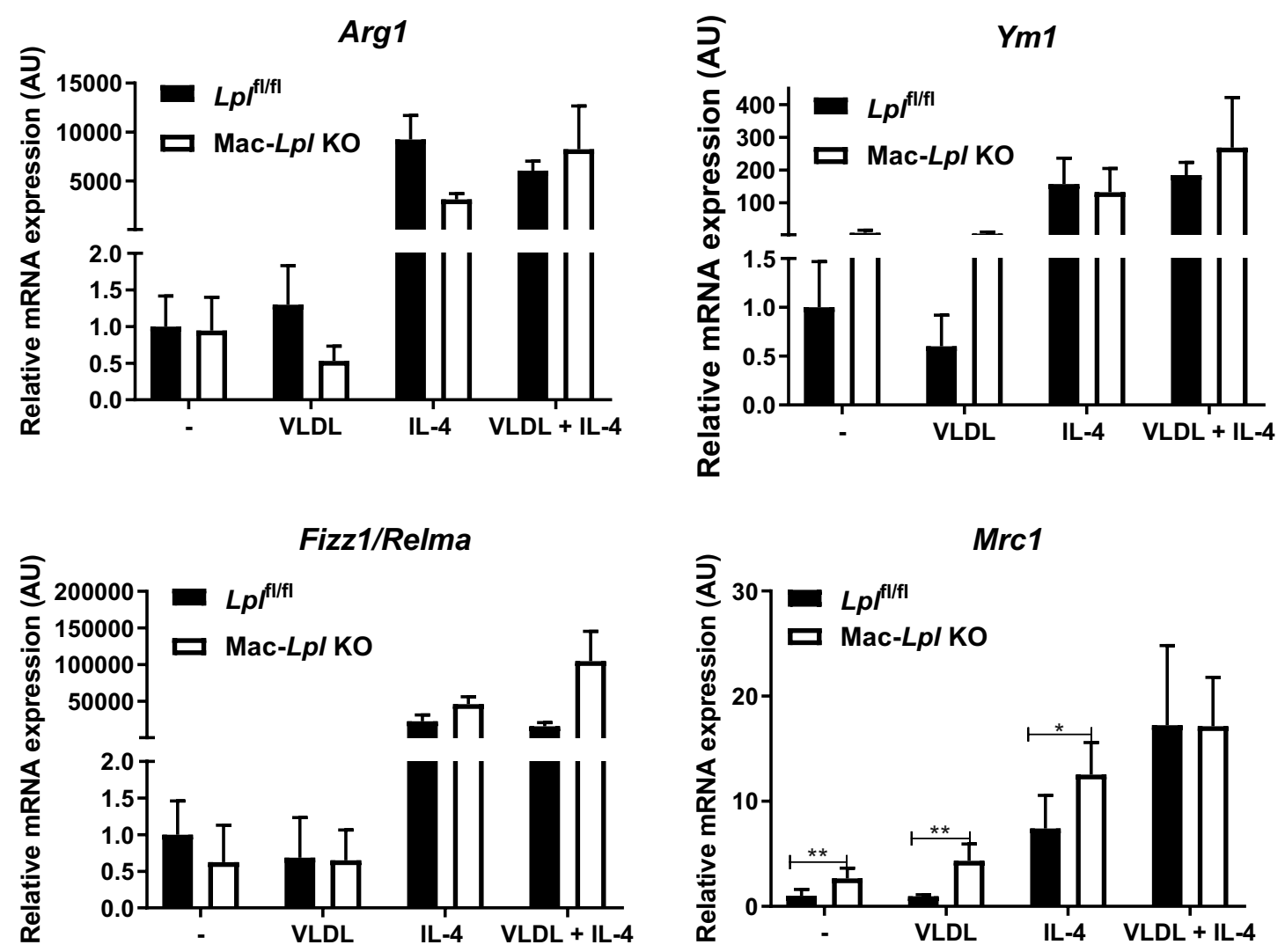

\section{Supplemental Figure X. BMDM culture in low $(1 \mathrm{mM})$ glucose}

Side-by-side comparison of anti-inflammatory genes from Figure 1 and $2 . \mathrm{N}=6 /$ group. ${ }^{*} \mathrm{p}<0.05,{ }^{* *} \mathrm{p}<0.01$. Results are represented mean \pm SD and compared using Two-way ANOVA, Sidak's multiple comparison. 

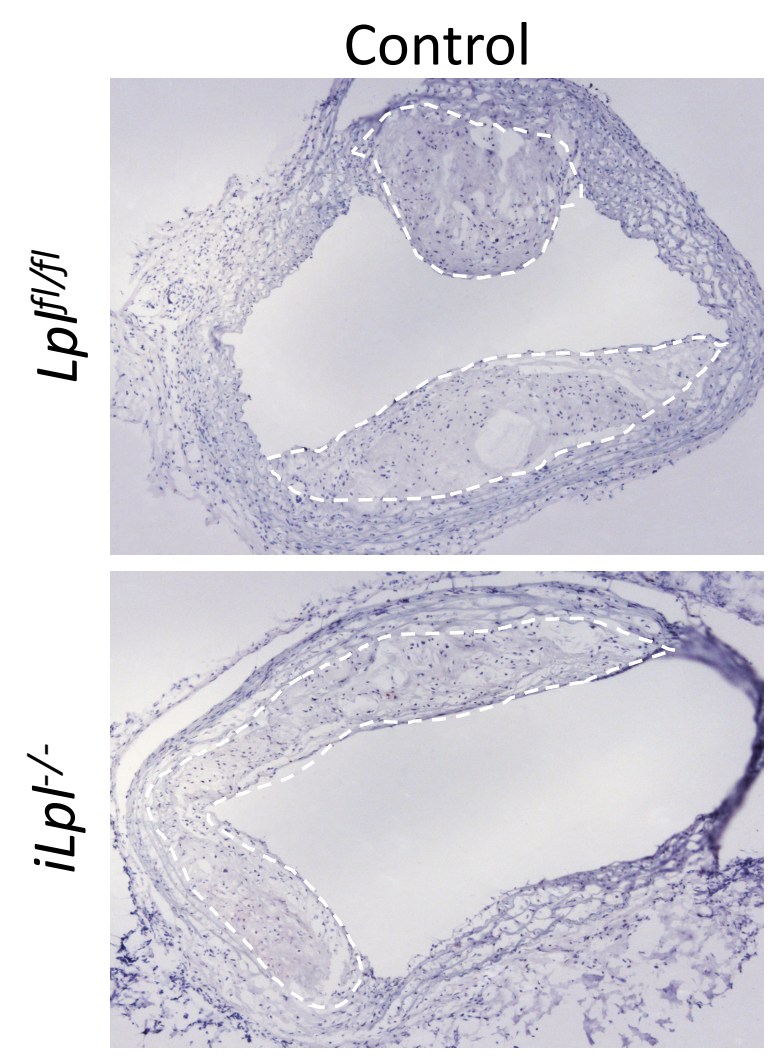

\section{Supplemental Figure XI. Control staining for CD68 and CD206}

Negative controls were performed using an isotype control (Rat lgG2a) instead of the primary antibody. 


\title{
4
}

\section{Reduced High-density Lipoprotein Cholesterol and Particle Number due to Hypertriglyceridemia does not affect Atherosclerosis Regression}

\author{
Tatjana Josefs, Debapriya Basu, Tomas Vaisar, Britt Arets, Jenny E. Kanter, Lesley-Ann Huggins, \\ Yunying $\mathrm{Hu}$, Jianhua Liu, Jay W. Heinecke, Karin E. Bornfeldt, \\ Ira J. Goldberg*, Edward A. Fisher*
}

${ }^{*}$ Equal contribution

In Revision 


\begin{abstract}
Rationale: Hypertriglyceridemia (HyperTG) and low high-density lipoprotein cholesterol (HDL-C), both of which are regulated by lipoprotein lipase (LpL) activity, associate with increased cardiovascular disease (CVD). Genetic regulators of LpL actions track with CVD risk in humans. Whether this is due to changes in HDL-C or function, or circulating triglyceride (TG) levels is unresolved.
\end{abstract}

Objective: We created HyperTG and HDL-C reduction in atherosclerotic mice to allow the assessment of how HyperTG and reduced HDL affect regression of atherosclerosis and the phenotype of plaque macrophages.

Methods and Results: Atherosclerosis regression was studied in control LpL floxed $\left(L p f^{f / f f}\right)$ mice and tamoxifen inducible whole-body LpL KO (iLp/ $\left.{ }^{-/-}\right)$mice with HyperTG $\sim 500 \mathrm{mg} / \mathrm{dL}$ and reduced HDL-C ( $\sim 50 \%$ reduction). Atherosclerosis regression was studied using two models: 1) aortic transplant, and 2) inhibition of the LDL receptor using antisense oligonucleotides followed by re-expression after sense oligonucleotide treatment. In a subset of mice, we expressed cholesterol ester transfer protein (hCETP) to humanize the relationship between apoB-lipoproteins and HDL. HDL particle number (HDL-P), cholesterol efflux capacity (CEC) and HDL proteome were measured in HyperTG mice and humans. Surprisingly, HyperTG and reduced HDL-C levels due to loss of $\mathrm{LpL}$ did not affect atherosclerosis lesion size or macrophage content (CD68+ cells) in either model. Expression of hCETP and further reduction of HDL-C did not alter lesions. Sera from iL $\mathrm{pl}^{--}$mice had a decrease in total CEC, but not ABCA1-mediated CEC. HyperTG humans, including those with LpL deficiency, had greater ABCA1-mediated CEC and total CEC per HDL-P.

Conclusion: Atherosclerosis regression is driven by LDL reduction and is not affected by HyperTG and HDL-C levels. 


\section{Introduction}

Although high-density lipoprotein cholesterol (HDL-C) levels are inversely correlated with cardiovascular disease (CVD), recent genetic studies have questioned whether this correlation reflects causation. ${ }^{1,2}$ Fasting levels of HDL-C and triglyceride (TG) are inversely related, thus it is difficult to dissect the importance of each of these lipids. Postprandial TG excursions also inversely correlate with HDL-C ${ }^{3,4}$ and some investigators consider HDL-C as a marker for average circulating TG levels. ${ }^{2}$ While several recent genome-wide association studies (GWAS) failed to directly link HDL modulating genes with CVD, genes affecting TG levels-lipoprotein lipase (LpL) and angiopoietin-like proteins 3 and 4 -do associate with clinical CVD endpoints. ${ }^{1}$

There are limited experimental models linking hypertriglyceridemia (HyperTG) and atherosclerosis, but a large series of experimental studies have documented benefits of raising HDL-C and apolipoprotein A1 (ApoA-I levels; reviewed in ${ }^{5,6}$ ). For example, preclinical studies have shown that increased expression of ApoA-I, the major protein in $\mathrm{HDL}$, reduces atherosclerosis ${ }^{6-8}$ and normalizes the defect in atherosclerosis regression that occurs with diabetes ${ }^{9}$. Some clinical studies have infused HDL or HDLlike particles and showed reduced atherosclerosis development ${ }^{10}$ with acute beneficial changes in plaque composition (e.g. reduced lipid and macrophage content) ${ }^{11}$. Methods to markedly induce ApoA-I expression in humans are not available. Other methods to increase HDL-C, such as blocking the exchange of cholesterol and TG between TG-rich lipoproteins and HDL by cholesteryl ester transfer protein (CETP) inhibition, were not beneficial when corrected for the expected benefits of LDL-C reduction by this treatment. ${ }^{12-14}$

One postulated reason for the lack of benefits with CETP inhibition in humans is that the increase in HDL-C does not reflect an increase in cholesterol efflux capacity (CEC) or reverse cholesterol transport (RCT). In humans, CEC has been touted as a better CVD risk marker than HDL-C. ${ }^{15-17}$ Moreover, the regression of lesions after marked LDL-C reduction is widely believed to rely on HDL-CEC to remove accumulated cholesterol. However, kinetic studies performed nearly 4 decades ago showed that the variation in HDL-C levels found in most humans did not affect overall cholesterol 
balance, which implied that CEC does not differ among most people. ${ }^{18}$ Thus, the role of HDL particles and their functionality in atherosclerosis are unclear.

In the present report, we tested whether reduced HDL-C due to HyperTG resulting from LpL deficiency would affect atherosclerosis regression, as well as CEC, in mice and humans. We hypothesized that LpL-deficiency would decrease CEC by reducing HDL particle numbers (HDL-P) and that this in turn would impair atherosclerosis regression. We chose to study regression, in part, because during this process, cholesterol must exit from atherosclerotic plaques, the first step of CEC. Moreover, current approaches to CVD in humans have a greater focus on the resolution of atherosclerotic coronary lesions through marked cholesterol reduction.

Our surprising results show that though LpL deficiency induced a reduction in HDL-C, the now TG-rich HDL particles maintained CEC. This likely allowed normal atherosclerosis regression and illustrates that normal repair of lesions can occur in the setting of HyperTG and reduced HDL-C.

\section{Materials and Method}

Animals: Global inducible LpL knockout mice were generated by crossing floxed $L p l\left(L p f^{f / f l}\right)$ mice ${ }^{19}$ with $\beta$-actin driven tamoxifen-inducible Cre (MerCreMer) transgenic mice (Jackson Laboratory) to obtain the $\beta$-actin-MerCreMer/LpL ${ }^{\text {flffl }}$ offspring designated as $\mathrm{i} L \mathrm{pH}^{--}$mice, as previously described. ${ }^{20}$ Mice received intraperitoneal (i.p.) injections of tamoxifen (Toronto Research Chemicals, \#T006000) or 4-hydroxytamoxifen (Tocris) in corn oil (Sigma) at a dose of $40 \mathrm{mg} / \mathrm{kg} \mathrm{BW/day} \mathrm{for} 5$ consecutive days. On the day of sacrifice, mice were anesthetized via intraperitoneal (i.p.) injection of ketamine $(100 \mathrm{mg} / \mathrm{kg})$ and xylazine $(10 \mathrm{mg} / \mathrm{kg})$. Blood was collected via cardiac puncture in EDTA-containing tubes and mice were perfused with $10 \%$ sucrose in saline solution $(0.9 \% \mathrm{NaCl})$. All procedures were approved by the Institutional Animal Care and Use Committee at New York University Langone Health approved protocol \#160907 (“Lipoprotein Lipase and ApoB”). Mice were maintained in a temperature-controlled $\left(25^{\circ} \mathrm{C}\right)$ facility with a $12 \mathrm{~h}$-light/dark cycle and given free access to water and food, except when fasting blood was obtained. Mice were fed a laboratory 
rodent chow diet or western diet (WD; 0.3\% cholesterol; Dyets Inc. D101977Gi) as indicated.

Plasma lipid measurements: Total cholesterol (TC) was measured using an enzymatic assay (Total Cholesterol E Kit, Wako Life Science, cat \# NC9138103 or Infinity Total Cholesterol Reagent, Thermo Scientific, cat \#TR13521). Plasma TG was measured using either enzymatic assay (L-Type Triglyceride M, Wako Life Science or Infinity Triglycerides, Thermo Scientific, cat \#TR2242) or a fluorometric kit (BioVision, \#K622-100).

Lipoprotein fractionation: Lipoprotein fractions were obtained by sequential density ultracentrifugation as described before ${ }^{20}$, and TC and TG measured as described above. Briefly, equal amounts of mouse plasma (70-100 $\mu \mathrm{L})$ were used for sequential density ultracentrifugation to separate very-low-density lipoprotein (VLDL $\mathrm{d}<1.006 \mathrm{~g} / \mathrm{mL})$, LDL $(\mathrm{d}=1.006-1.063 \mathrm{~g} / \mathrm{mL})$, and HDL $(\mathrm{d}=1.063-1.21 \mathrm{~g} / \mathrm{mL})$ in a TLA 100 rotor (Beckmann Instruments, Palo Alto, CA).

hCETP: hCETP expression was induced by injecting mice i.p. with either adenoassociated virus (AAV) hCETP (AAV8.TBG.PI.hCETP.SV40) or a LacZ control virus (AAV8.TBG.PI.LacZ.bGH) at a concentration of $5 \times 10^{11} \mathrm{GC}$ (genome copies)/mouse (Penn Vector Core) as done before. ${ }^{21}$ Plasma CETP activity was measured using a CETP Activity Assay Kit (Sigma Aldrich, MAK106). CETP expression in the liver was assessed by qPCR from homogenized liver samples (Primer sequence in Supplemental Table I).

Aortic transplant regression model: The aortic transplant model has been described before. $^{22-25}$ Briefly, a plaque burdened aortic arch from a hypercholesterolemic LdIr/mouse (fed a WD for 16wks) was transplanted into a normocholesterolemic recipient mouse (iLp $\mathrm{F}^{-/}$or $\left.L p /^{f / f f}\right)$, interpositioned with the abdominal aorta and blood flow was directed through the graft. All recipient mice were $\sim 22$ weeks old and maintained on standard chow diet until sacrificed 14 days after aortic transplantation. 
LDL receptor (LDLR) antisense oligonucleotide (ASO) regression model: This protocol was based on our published model. ${ }^{38}$ Briefly, GalNAc-conjugated Gen 2.5 ASO targeting mouse LDLR was developed and provided by lonis Pharmaceuticals. LDLR ASO was injected intraperitoneally at a dose of $5 \mathrm{mg} / \mathrm{kg}$ body weight once a week for 16 weeks. GalNAc-conjugated sense oligonucleotide (SO) designed to bind and inactivate the LDLR ASO was injected once i.p. at a dose of 20 $\mathrm{mg} / \mathrm{kg}$ body weight to accelerate the return of LDLR expression.

Bone marrow transplant (BMT): Bone marrow cells were isolated from C57BL/6 mice by flushing tibiae and femur bones, followed by red blood cell lysis and BM cells suspended in DMEM (Dulbecco's Modified Eagle Media, Fisher Scientific). 5*106 cells/mouse were injected retro-orbitally into 8 weeks old radiated $(10.000 \mathrm{~Gy}) \mathrm{iLpl}^{-/-}$ and $L p f^{f / f l}$, followed by 4 weeks of antibiotic treatment administered in drinking water.

Atherosclerotic plaque assessment: Aortic arches were removed after perfusion with saline $+10 \%$ sucrose, embedded in OCT (TissueTEK) and frozen at $-80^{\circ} \mathrm{C}$. Serial sections (6 $\mu \mathrm{m}$ thick) were cut using a cryostat and stained for CD68 (Bio-Rad, Cat. MCA1957) ${ }^{25}$, inducible nitric oxide synthase (iNOS; Abcam, Cat. ab209027) ${ }^{26}$ and Arginase1 (Thermo Fisher Scientific, Cat. 53-3697-82) to determine content and phenotype of macrophages. For further plaque characteristics, Movats ${ }^{27}$ and Picrosirius Red (Abcam, Cat. ab246832) ${ }^{28}$ stainings were performed as described previously. Picrosirius Red staining was visualized using a polarized light microscope (Zeiss Axio Observer) to determine collagen ${ }^{+}$areas.

Aortic roots and brachiocephalic arteries (BCAs) were removed after perfusion with PBS. The root was embedded in OCT (TissueTEK), frozen, and stored at $-80^{\circ} \mathrm{C}$. Serial sections $(6 \mu \mathrm{m})$ of roots were obtained by cryosectioning, followed by Mac- $2^{29}$ or $\mathrm{CD}^{25} 8^{25}$ immunostaining to determine macrophage content as described. BCAs were collected in $10 \%$ formalin, kept overnight at $4^{\circ} \mathrm{C}$, and stored in $70 \%$ ethanol at $4^{\circ} \mathrm{C}$ for further processing. BCAs embedded in paraffin were sectioned $(5 \mu \mathrm{m})$ and every fifth cross section stained using the Movat pentachrome method. The maximal lesion site was determined, and adjacent sections were immunostained using a Mac-2 antibody to detect lesion macrophages, as described previously. ${ }^{29}$ 
ImagePro Plus 7.0 software was used to determine $\mathrm{CD}^{+} 8^{+}, \mathrm{Mac}^{+}{ }^{+}$and collagen ${ }^{+}$area. Image J $1.51 \mathrm{r}(\mathrm{NIH})$ was used to determine iNOS ${ }^{+}$, Arginase $1^{+}$cells and divided by total cells (DAPI nuclear staining) to get percentage of total cells. Acellular areas were measured to determine necrotic core area and fibrous cap was evaluated as none, very thin (<5layers), thin (5-10 layers) and thick (>10 layers) above the necrotic core using Movats stain.

Macrophage phenotyping: CD68+ cells were selected from atherosclerotic plaques from $i L \mathrm{pl}^{-/-}$and $L p \mathrm{I}^{\mathrm{fl} / \mathrm{fl}}$ mice by laser capture microdissection (LCM) as previously described. ${ }^{30,31}$ Briefly, $6 \mu \mathrm{m}$ frozen sections from aortic arches were stained with hematoxylin-eosin and foam cells identified under a microscope and verified by positive CD68 staining. All LCM procedures were performed under RNase-free conditions. After LCM, RNA was isolated using the PicoPure Kit (Molecular Devices, Inc., Sunnyvale, CA), treated with DNase and the quality and quantity determined using an Agilent 2100 Bioanalyzer (Agilent Technologies, Santa Clara, CA). RNA was converted to cDNA and amplified using the WT-Ovation Pico RNA Amplification Kit (NuGEN, San Carlos, CA). Data were normalized to HRPT with the $\triangle \triangle \mathrm{C}(\mathrm{t})$ method and are presented as relative transcript levels. All primers are listed in Supplemental Table I.

HDL analyses: Cholesterol efflux capacity (CEC) was analyzed by methods based on methodology developed by Rothblat et al as described previously. ${ }^{32-34}$ Briefly, cAMPstimulated $\mathrm{J} 774$ cells were radiolabeled with $\left[{ }^{3} \mathrm{H}\right]$-cholesterol and incubated with ApoB depleted plasma for $4 \mathrm{~h}$. CEC was quantified as the percentage of label in the medium relative to total labelled cholesterol levels in the culture system after subtraction of values obtained without $A$ poB depleted serum. ABCA1-mediated CEC was determined by analogous method in BHK cells expressing ABCA1 under mifepristone control where the ABCA1-specific CEC represents the difference between CEC of mifepristone stimulated and non-stimulated BHK cells.

HDL particle number (HDL-P) and particle size distribution was quantified using calibrated differential ion mobility analysis. ${ }^{33,35}$ Proteome of HDL isolated by sequential density ultracentrifugation was characterized and quantified using data independent 
analysis (DIA) mass spectrometry. ${ }^{34,36}$ Proteomics data were analyzed using Reactome (https://reactome.org). ${ }^{37}$

Human subjects: Plasma was obtained from 9 donors with elevated plasma TG levels of which three were LpL deficient (Characteristics in Supplemental Table VI), and 6 control subjects with normal plasma TG levels. CEC, HDL-P and HDL proteomics analyses were performed as described above.

Statistical Analyses: Data are expressed as mean \pm SEM. Data were tested on normality and equal variance and analyzed by appropriate parametric or nonparametric test as stated in each figure. $p \leq 0.05$ was considered significant (GraphPad Prism 7).

\section{Results}

LpL deficiency increased circulating VLDL-TG and reduced HDL-C, which was further reduced with hCETP expression

At $\sim 5$ weeks of age, LdIr $r^{/-}$mice were weaned onto WD, which was continued for 16 weeks to promote advanced atherosclerotic lesion formation (baseline group; Figure 1A, Supplemental Figure IA). Plaque-bearing aortic grafts were then transplanted into $L p I^{f l / f l}$ (control) and $i L \mathrm{pl}^{-/-}$mice (HyperTG, induced 3 weeks prior to transplantation) that were normocholesterolemic. Both recipient groups had been maintained on a standard laboratory diet prior to receiving aortic transplants; this diet was continued for 2 weeks to maintain normocholesterolemia and induce plaque regression. A subset of mice was injected with an AAV hCETP $\left(5 \times 10^{11}\right.$ genome copies/mouse; Figure $\left.1 \mathrm{~A}, \mathrm{D}\right)$ to investigate whether the absence of CETP expression in mice was a factor in the effects of HyperTG.

In contrast to the hypercholesterolemic baseline/donor mice (TC $867 \pm 36$ $\mathrm{mg} / \mathrm{dL}$ ), both aortic transplant recipient groups were normocholesterolemic (TC $79 \pm$ $10 \mathrm{mg} / \mathrm{dL}$; Figure 1B). Plasma TG levels were normal in $L p f^{f / f l}$ mice ( 67 mg/dL) and, as expected, TG levels were elevated in $i L p{ }^{-/-}$mice ( $461 \mathrm{mg} / \mathrm{dL}$; Figure $\left.1 \mathrm{C}\right)$. hCETP injection did not affect TC or total TG levels but led to an expected increase in circulating CETP activity (Figure 1D). Body weights of the mice did not change due to 
the transplant procedure and were similar in all regression groups (Supplemental Figure IB). Body weight was elevated in the baseline group due to WD feeding.

The changes in lipoprotein profile due to LpL deficiency and hCETP expression are shown in Figures 1E-F. The most remarkable lipid change that occurred with hCETP and HyperTG was a marked reduction in HDL-C with smaller changes in other lipoprotein parameters. VLDL-C increased in $i L \mathrm{pl}^{/-}$compared to $L p f^{f / / f l}$ mice $\left(i L p F^{-/-} 37 \pm\right.$ 7 versus $\left.L p f^{f / f f l} 13 \pm 3 \mathrm{mg} / \mathrm{dL}, P=0.04\right)$. As $L p L$-mediated lipolysis is required for $L D L$ and HDL formation, LpL deficient mice had decreased LDL-C and HDL-C levels; HDLC was decreased from $29 \pm 2$ to $17 \pm 1 \mathrm{mg} / \mathrm{dL}(P=0.004)$ and even further reduced with hCETP expression to $9 \pm 2 \mathrm{mg} / \mathrm{dL}(P<0.0001)$. As expected, VLDL-TG was increased in $i L p F^{-/-}$compared to $L p f^{f / f f}$ mice (iLp/ ${ }^{-/} 314 \pm 40$ vs. $L p f^{f / f f l} 60 \pm 15 \mathrm{mg} / \mathrm{dL}, \mathrm{P}$ $<0.0001$, Figure 1F). Neither LDL-TG nor HDL-TG differed between $L p^{f / / f l}$ and $i L \mathrm{pl}^{-/-}$ mice, but HDL-TG significantly increased with hCETP expression to the same extent in mice with and without $L p$ / expression (average HDL-TG with hCETP $3.3 \pm 1.4 \mathrm{mg} / \mathrm{dL}$ vs. no hCETP $1.2 \pm 0.2 \mathrm{mg} / \mathrm{dL}$ ). Thus, LpL deficiency induced HyperTG and reduced LDL-C and HDL-C. hCETP reduced HDL-C even further and created TG-enriched HDL as expected from its role in humans in exchanging VLDL-TG for HDL-C. 

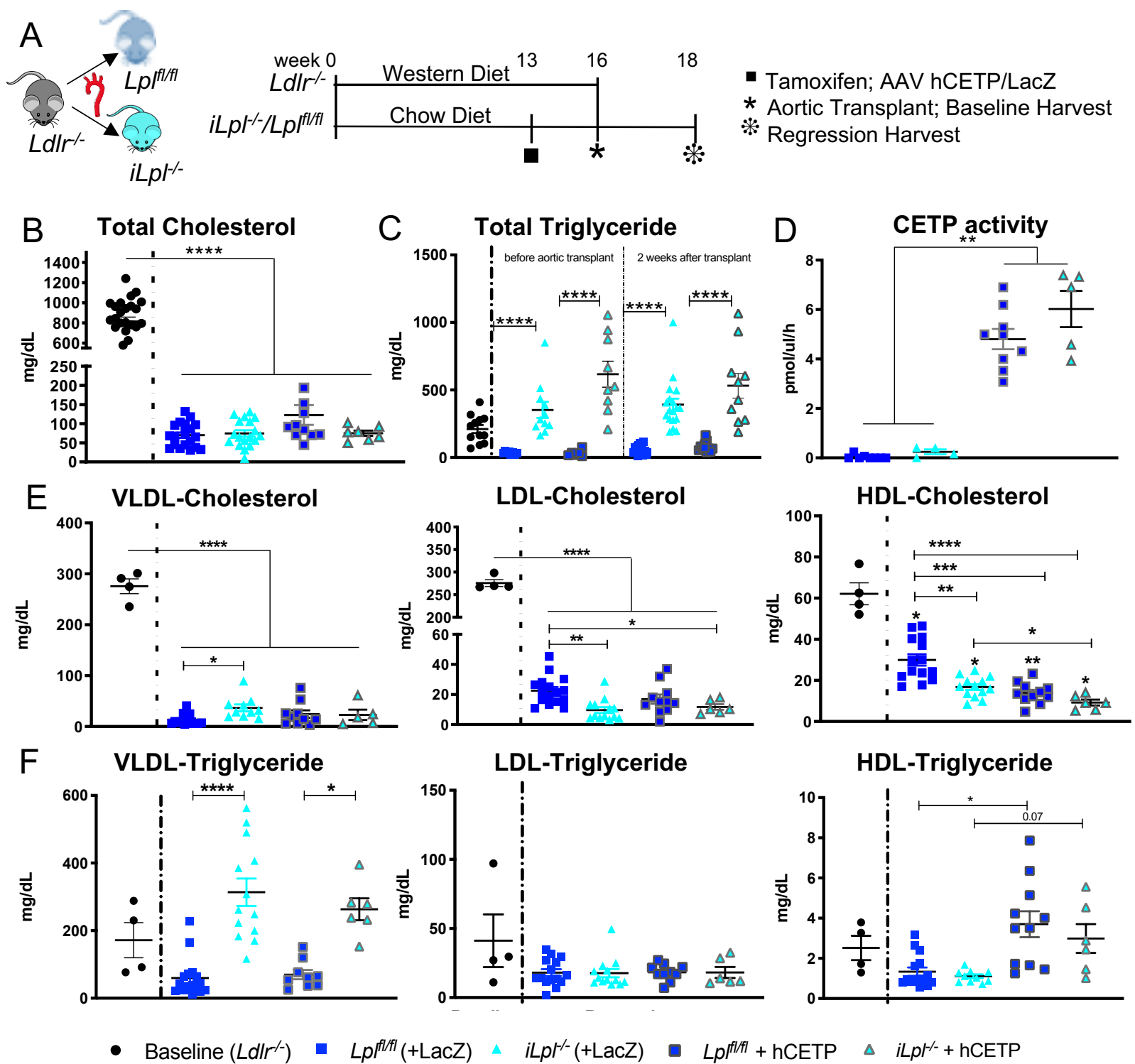

Figure 1. LpL deficiency increased VLDL-TG and reduced HDL-C, which was further reduced with hCETP expression. (A) Study Setup (B) Total plasma cholesterol levels at time of harvest (C) Total triglyceride (TG) levels in baseline and regression groups before and after the 2 weeks atherosclerosis regression period (D) CETP activity measured in circulation. Cholesterol $(E)$ and triglyceride levels $(F)$ of isolated lipoproteins. $N=5-24 /$ group; data represented as mean $\pm \mathrm{SEM},{ }^{*} \mathrm{P}<0.05,{ }^{* *} \mathrm{P}<0.01,{ }^{* * *} \mathrm{P}<0.001,{ }^{* * * *} \mathrm{P}<0.0001,1$-way ANOVA with Tukey's multiple comparison test; HDL-C and HDL-TG Welch ANOVA with Games-Howell's multiple comparison test; VLDL-TG \& LDL-TG Kruskal-Wallis Test with Dunn's multiple comparison test 
HyperTG due to global LpL deficiency does not affect atherosclerosis regression in the aortic transplant mouse model

After two-weeks of plaque exposure to normocholesterolemia (atherosclerosis regression period), lesion areas were similar in all groups (Figure 2A-B). We considered as evidence of atherosclerosis regression decreases in lesion area or macrophage content because the two parameters can be independent of each other (e.g. ${ }^{38}$ ) and either is considered to be a beneficial change.

The macrophage content (CD68+ area, Figure 2C) decreased in regression groups compared to baseline, but did not differ between mice with and without LpL/hCETP expression (baseline $21.4 \% \pm 1.3$ versus average of 4 regression groups: $11.5 \% \pm 1.4, \mathrm{P}<0.0001)$. To further evaluate the plaques, we assessed necrotic core, fibrous cap and collagen. Necrotic core (\% lesion area) did not significantly differ between groups (Figure 2A, C-D). Fibrous caps (Figure 2E) and collagen (Figure 2G) were increased compared to baseline $(P=0.052)$, but did not significantly differ between regression groups. Therefore, the favorable remodeling of the cellular composition of the plaques (in particular, reduced macrophage content, an index of the resolution of lesion inflammation) was not impaired by HyperTG and/or hCETP expression. Moreover, we could not correlate lesion area or macrophage content with the degree of HyperTG (Supplemental Figure II).

HyperTG due to non-macrophage LpL deficiency also does not affect atherosclerosis regression in the aortic transplant mouse model

LpL is also expressed by macrophages and has been shown to promote lipid uptake and foam cell formation. ${ }^{39-41}$ To exclude possible counterbalancing effects of the putative atherogenic HyperTG and atheroprotective LpL deficiency in macrophages, we performed atherosclerosis regression studies using $L p f^{f / f f l}$ and $i L p F^{-/-}$transplanted with WT bone marrow (Supplemental Figure III A-B). HyperTG mice with LpL expressing macrophages showed body weights and lipid levels similar to those in total LpL-deficient mice (Supplemental Figure III C-G). Unlike the effects of macrophage LpL to promote atherosclerosis ${ }^{39}, 42$, the number of macrophages per lesion area (\%CD68) during regression was similarly reduced in mice with and without macrophage LpL expression, showing a reduction of $>50 \%$ compared to baseline (Supplemental Figure III H-I). Thus, knocking out macrophage LpL expression did not 
alter regression. These data are similar to another study that showed LpL expression does not modify lesional macrophage phenotype in the setting of atherosclerosis regression. ${ }^{20}$
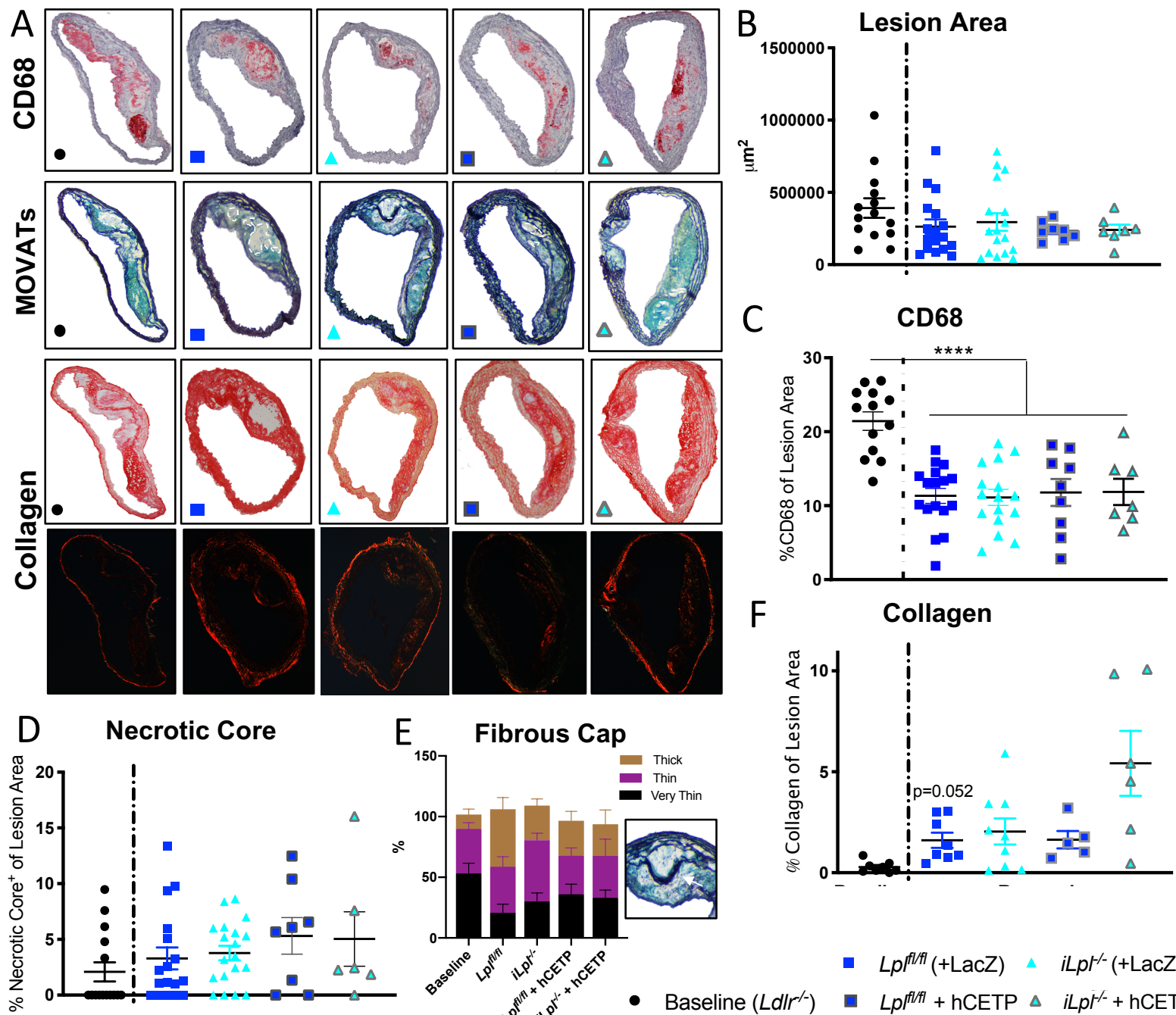

C

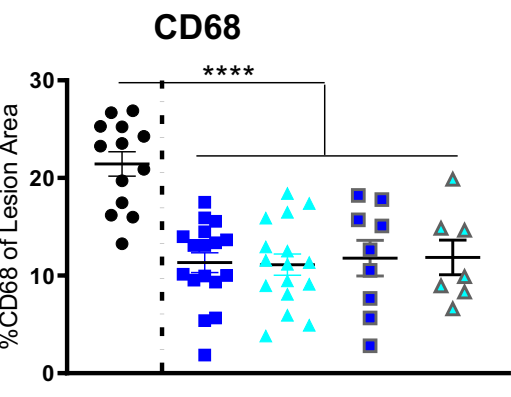

$\mathrm{F}$

Collagen

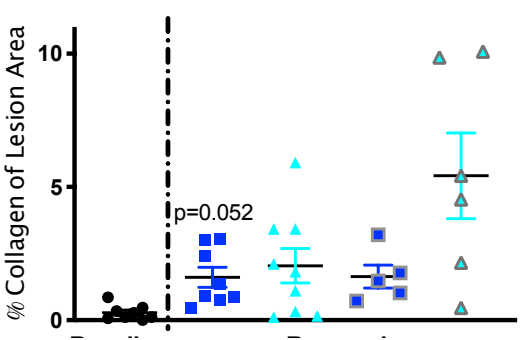

Figure 2. LpL deficiency (+/- hCETP) does not impair atherosclerosis regression.

(A) Representative stainings; CD68, MOVATs, Pico Sirius Red Staining (brightfield \& polarized light). Quantification of (B) Lesion Area $\left(\mu \mathrm{m}^{2}\right)$ and (C) CD68 Area (\% of Lesion Area ) (D) Necrotic Core ( $\%$ of Lesion Area) (E) Fibrous Cap determined by number of layers above the Necrotic Core, Thick >10layers, Thin 5-10layers, Very Thin $<5$ layers ( $F$ ) Collagen (\% of Lesion Area) $\mathrm{N}=6$-19/group, data represented as mean \pm SEM, ${ }^{*} \mathrm{P}<0.0001$, 1-way ANOVA with Tukey's multiple comparison test; $(F)$ Welch ANOVA with Games-Howell's multiple comparison test 


\section{LpL deficiency mediated HyperTG does not affect atherosclerosis regression in a non-invasive mouse model}

To confirm the results on $\mathrm{LpL}$ and atherosclerosis regression in an independent model (Figure 3A), WD-fed $L p f^{f / f l}$ and $i L p l^{-/}$(non-induced) mice were made hypercholesterolemic using LDLR ASO treatment for 16 weeks. At the end of 16 weeks, the ASO treatments stopped and one subset from each genotype was analyzed as the 'baseline'. The other subset of mice was treated with tamoxifen to induce LpL deletion at week 14 . These mice were then treated with LDLR SO at week 16 to rapidly reverse hypercholesterolemia and to induce regression. ${ }^{43}$ After another 3 weeks, these mice were studied to analyze the regressing plaques. As expected, plasma TC decreased in regression groups compared to baseline (Figure 3B), and plasma TG increased significantly in $i L p \mathrm{~F}^{-/-}$mice (Figure $3 \mathrm{C}$ ).

As with the transplant model, with marked cholesterol reduction there were no changes in total plaque areas in the aortic root (Figure 3D). There was a marked reduction in CD68\% area (Figure 3E), but HyperTG did not affect this. In parallel, we noted no changes in total plaque area in the BCAs (Figure 3F), but as in the root, there was a decrease in macrophage area (Figure 3G, $P<0.05$ ). Again, HyperTG did not affect the extent of BCA regression.

Thus, in two independent models of atherosclerosis regression, HyperTG iLp/-mice had similar changes as those in the control $L p f^{f / f l}$ mice after reversal of hypercholesterolemia, and in the LDL ASO model there was no evidence that the changes we observed were specific only to the aortic root.

\section{LpL deficiency-mediated HyperTG with local lipolysis in plaques does not affect atherosclerosis regression in a non-invasive mouse model of regression}

One possible cause for the postulated atherogenicity of HyperTG is the generation of local lipolysis products. ${ }^{44-46} \mathrm{iLpl}^{-/-}$mice would be protected from this type of injury due to generalized LpL deficiency. To determine the importance of local lipolysis we assessed atherosclerosis regression in mice with LpL expression in endothelial cells driven by a Tie2-Lpl transgene, described $\mathrm{in}^{47}$. These mice were allowed to develop atherosclerosis using the LDLR ASO protocol ${ }^{43}$ and regression was induced by reversing the hypercholesterolemia with SO. Total plasma cholesterol significantly decreased in regression groups after SO treatment (Supplemental Fig IV A). Mice with 
total LpL deficiency and the Tie2-LPL transgene had mild HyperTG compared to LpL floxed mice with the Tie2-LPL transgene (Supplemental Figure IV B). We quantified aortic root total plaque area (Supplemental Figure IV C) and percentage of plaque that was occupied by macrophages (Figure IV D) along with BCA total plaque area (Supplemental Figure IV E) and percentage of the Mac-2 positive (i.e., macrophage positive) area in BCA plaques (Supplemental Figure IV F). There were no significant differences attributable to increasing the lipolytic capacity of the endothelial cells. Thus, greater local lipolysis did not alter regression.
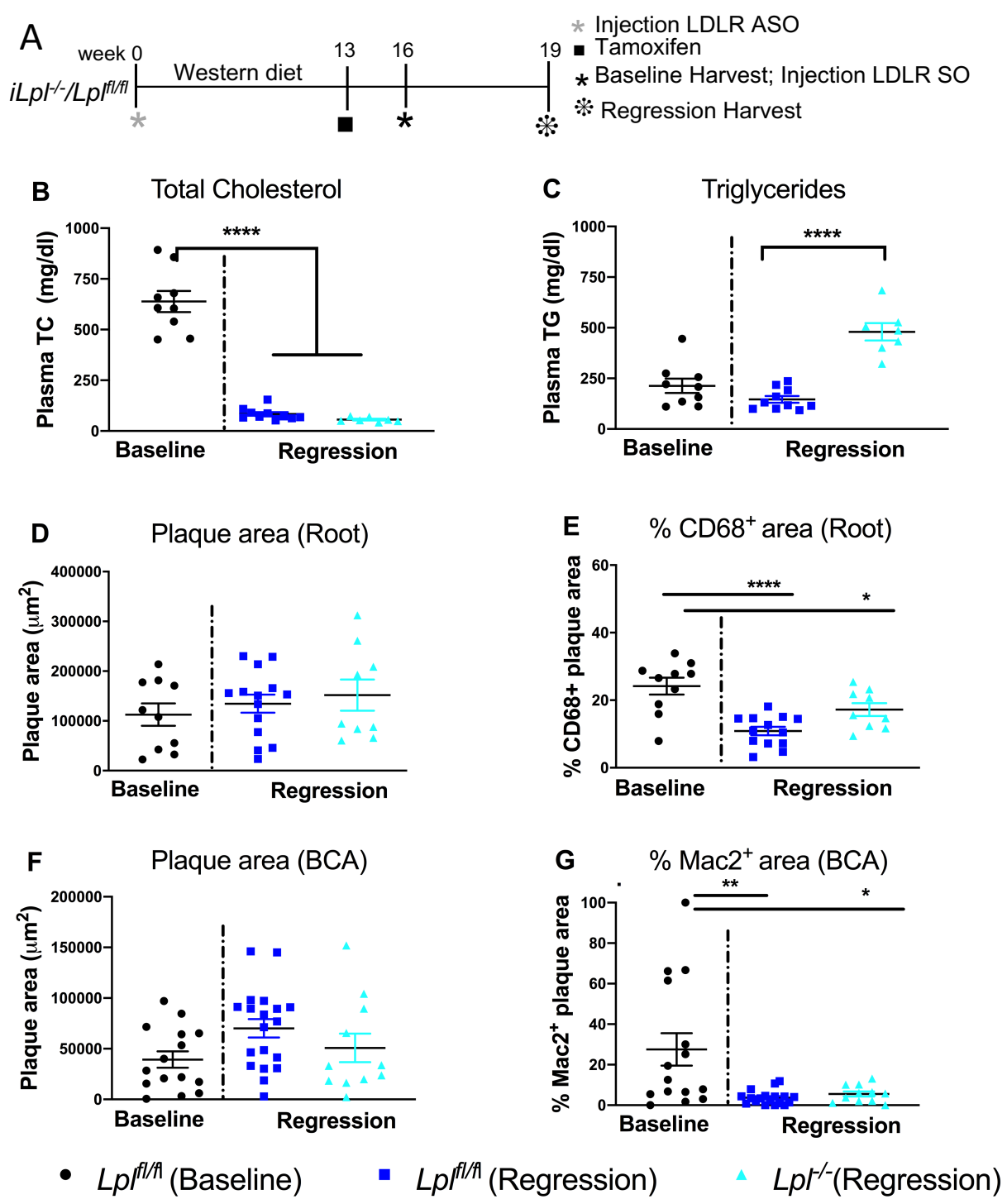

Figure 3. LpL deletion does not alter atherosclerosis regression in a noninvasive model of regression. 
(A) Study design: atherosclerosis was created in $L p f^{f / f l}$ and $i L p r^{--}$mice with LDLR antisense oligonucleotides (ASO) and western diet feeding for 16 weeks. One set of mice were analyzed at 16 weeks as the baseline group and the rest of the mice were treated with $\mathrm{SO}$ to induce regression and were analyzed after 3 weeks. The mice in regression group were also treated with tamoxifen at Week 13 to induce hypertriglyceridemia in $i \mathrm{pl}^{-{ }^{-}}$mice. (B) Plasma total cholesterol (TC) and (C) triglyceride (TG) levels in baseline and regression groups. (D) Total plaque area, (E) $\%$ of macrophages $(\mathrm{CD68}+)$ within aortic root lesions, $(F)$ total plaque area, $(G) \%$ of macrophage (Mac2+) in plaques within the BCA in the baseline and regression groups. $n=7-15$ /group, results expressed as mean \pm SEM. * $P<0.05$, ${ }^{* *} P<0.01$ and ${ }^{* * * *} P<0.0001$ using 1 -way ANOVA with Tukey's multiple comparison test.

\section{Effects of HyperTG on plaque macrophage gene expression}

Given the similarity in the results between the two regression models in terms of changes in plaque macrophage content, we wondered if there may have been altered phenotype related to HyperTG. Thus, RNA expression of isolated plaque macrophages and immunofluorescence staining were performed using samples from the transplant studies (Figure 4).

We have previously shown that transcriptomic profiling of atherosclerotic macrophages did not disclose significant differences in metabolism-related genes or pro- or anti-inflammatory genes in LpL-expressing versus LpL-deficient macrophages. ${ }^{20}$ To determine if hCETP expression affected macrophage phenotype, we isolated $\mathrm{CD} 68+$ cells by laser capture micodissection from $L p f^{f / f f l}$ and $i L \mathrm{p}^{-/}$mice expressing hCETP. In line with the previous results, we did not observe significant changes in metabolism-related genes (plin2, cd36, glut1, fasn, cpt1a), pro-inflammatory genes (tnfa, nos2, mcp1), or anti-inflammatory genes ( $m r c 1$, fizz1, il10) (Figure 4A-C). Staining of arginase-1 (a marker of M2-like macrophages, Figure 4D-E) and iNOS (a marker of M1-like macrophages, Figure 4F-G) in atherosclerotic plaques showed no differences between $L \mathrm{p}^{f / f t l}$ and $\mathrm{i} L \mathrm{pl}^{-/}$mice expressing hCETP, further confirming transcriptomic profiling results. 


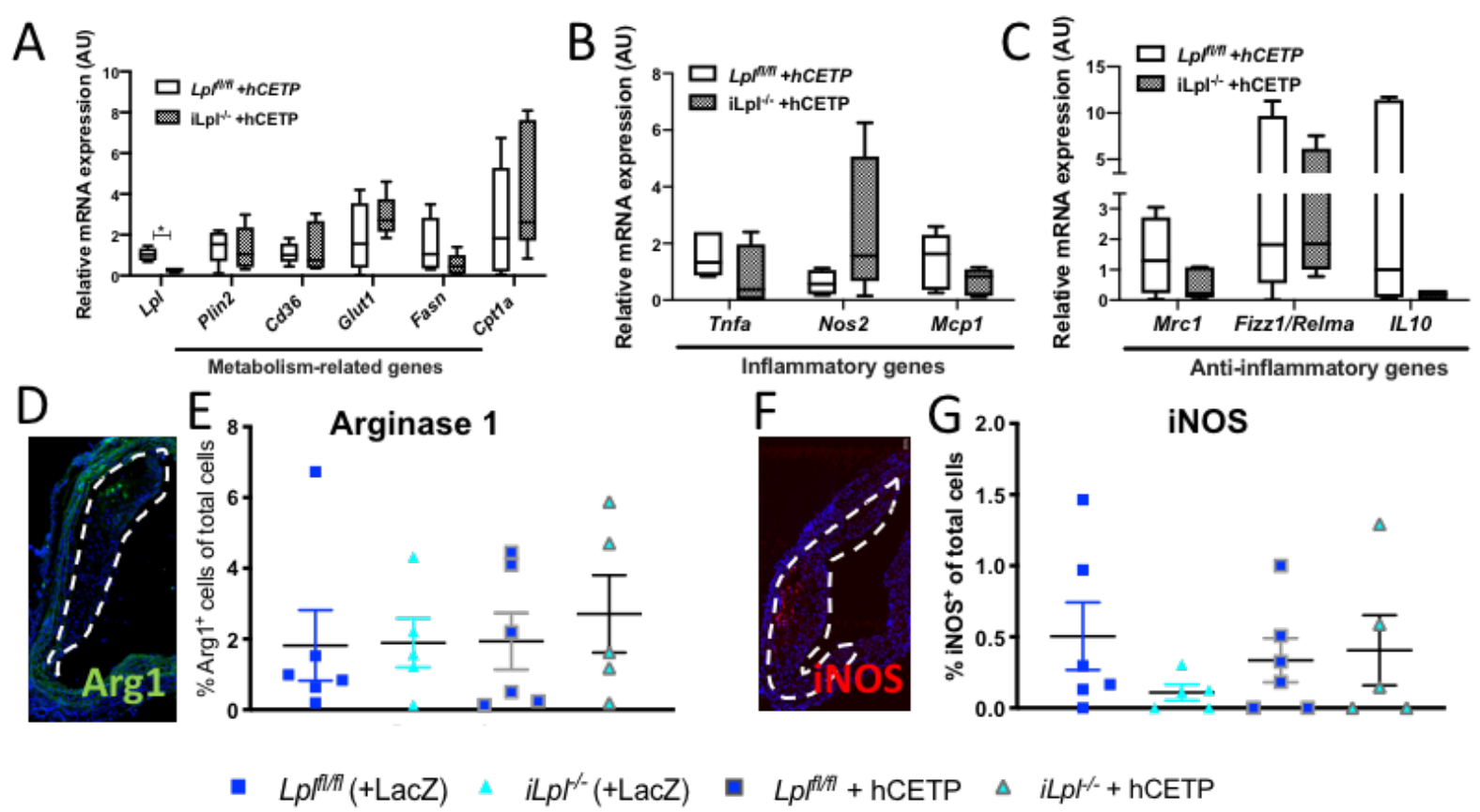

Figure 4. LpL deficiency (+/- hCETP) does not affect macrophage phenotype.

Macrophage phenotype was assessed by isolating mRNA from atherosclerotic CD68+ macrophages using Laser Capture Microdissection and mRNA expression measured for $(A)$ Metabolism-related genes (B) Inflammatory genes (C) Anti-inflammatory genes. Additionally, immunofluorescence staining of atherosclerotic arches was performed for (D-E) Arginase-1 (\%cells per total cells) and (F-G) iNOS (\%cells per total cells). $\mathrm{N}=5-6 /$ group, data represented as mean \pm SEM, * $P<0.05$, (A-C) unpaired t-test, $(E, G)$ 1-way ANOVA with Tukey's multiple comparison test

\section{LpL deficiency-mediated HyperTG reduces HDL-C and HDL-P, but does not} impair CEC

We hypothesized that regression was unchanged in our HyperTG mice because although HDL-C was reduced, CEC was maintained. To determine this, we assessed plasma levels of HDL-P and CEC. LpL deficiency leads to lower HDL-C levels in humans ${ }^{48}$ and in animal models ${ }^{49}$. We now show that $L p L$ deficiency also significantly lowers circulating HDL-P levels, which were further reduced by hCETP expression (Figure $5 \mathrm{~A}, \mathrm{P}<0.001$ ). Dividing total HDL-P into their subclasses according to size showed that the reduction was in small $(9.8-10 \mathrm{~nm})$ and medium $(10.4-11.1 \mathrm{~nm})$, but not in large $(11.9-12.6 \mathrm{~nm})$ HDL-P (Figure 5A).

We next measured CEC according the protocol developed by Rothblat and colleagues, in which radiolabeled cAMP-stimulated $\mathrm{J} 774$ cells are incubated with ApoB-depleted plasma for $4 \mathrm{~h}$, followed by quantification of labelled cholesterol in cells 
and medium. ${ }^{50}$ Total CEC and ABCA1 mediated CEC were assessed (Figure 5B). LpL deficiency decreased total CEC ( $L p f^{f / f f l} 7.5 \pm 0.4 \%$ vs. $\left.i L p /^{/-} 5.4 \pm 0.7 \%, P=0.0002\right)$, but despite the marked reduction in HDL-C, ABCA1-mediated efflux was not reduced. There was no reduction in total CEC or ABCA1-mediated efflux in mice expressing hCETP. When total CEC and ABCA1-mediated efflux on a per particle basis were calculated, there was no significant differences between $L p f^{f / f f l}$ and $i L p I^{-/-}$mice (Figure 5C). Thus, HyperTG did not produce dysfunctional HDL. hCETP and TG-enrichment of HDL (Figure 1G) increased total CEC per particle ( $L p f^{f / f f}+$ hCETP $1.30 \pm 0.06 \%$ vs. $\left.L p f^{f / f f l} 0.90 \pm 0.06 \%, P=0.018\right)$ and ABCA1-mediated efflux per particle in $L p f^{f / f f l}$ mice $\left(L p f^{f / f f}+\right.$ hCETP $8.36 \pm 0.62 \%$ vs. $\left.L p f^{f / f f l} 7.72 \pm 0.37 \%, P=0.014\right)$. Total CEC and ABCA1-mediated CEC per particle showed a trend towards an increase in $i \mathrm{~L} \mathrm{~F}^{-/}$mice expressing hCETP. These results show that despite a reduction in HDL-C and HDL-P with HyperTG, the unchanged regression correlated with maintained CEC.

\section{Effects of LpL and CETP on the HDL proteome}

We next determined how $\mathrm{LpL}$ deficiency expression altered the HDL proteome. Comparing HDL of $L p f^{f / / f l}$ with $i L{ }^{-/-}$mice, of the 133 detected proteins, 50 proteins were significantly different with 18 being down- and 32 up-regulated (Figure 5D-E, Supplemental Table II-III). Differentially detected proteins were mainly associated with the apoplipoprotein structural components, such as ApoA-I, ApoC-III, and ApoC-IV, but also included changes in PLTP and Angptl3 (Figure 5E). ApoA-I was reduced along with the reductions in HDL-C and HDL-P.

We next assessed how hCETP expression altered the proteome in $L p f^{f / f f}$ and $i L \mathrm{p}^{-/-}$mice. In mice expressing hCETP, we detected a total of 97 proteins, of which 15 proteins significantly differed (1 was down- and 14 up-regulated in iLp/ ${ }^{-/}+$hCETP; Figure 5F-G, Supplemental Table IV). Surprisingly, some of the proteins found in the HDL proteome from non-hCETP mice were not detected in hCETP-expressing mice, such as ApoA-V. Of the 133 detected proteins in non-hCETP expressing mice and 97 detected proteins in hCETP-expressing mice, eighty-five of the proteins were present in both groups. Of these 85 proteins, only 4 proteins ( 1 down- and 3 up-regulated) were significantly different with hCETP expression, showing that hCETP has modest effects on the mouse HDL proteome (Supplemental Table V). 

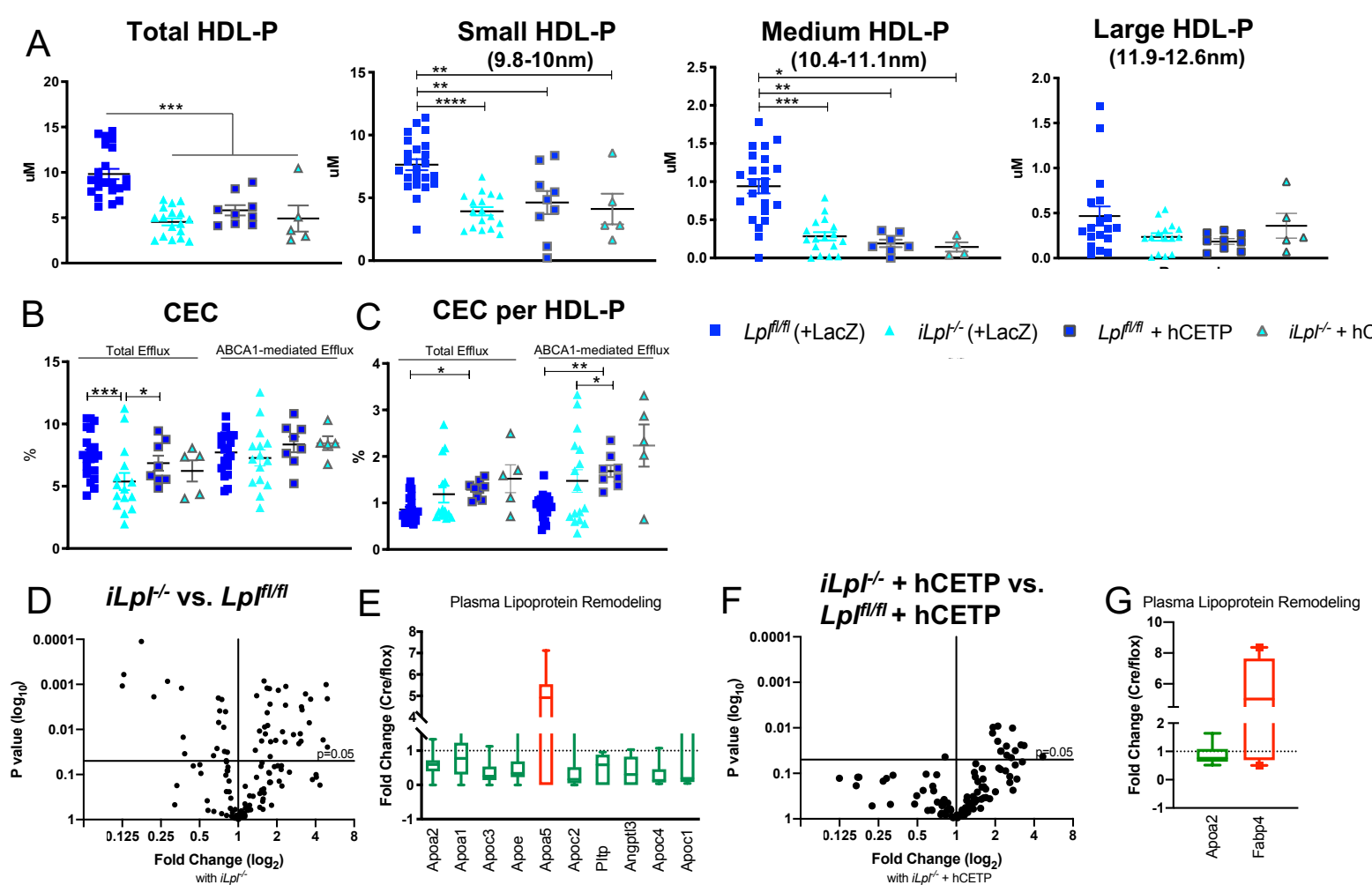

Figure 5. LpL deficiency decreases HDL-P, changes HDL Proteome, but does not change CEC with minor effects of hCETP.

(A) Total HDL-P and its subfractions (B) Total and ABCA1-mediated HDL Cholesterol Efflux Capacity (CEC) (C) Total and ABCA1-mediated CEC per HDL-P (D) Volcano blot of HDL Proteomics in $i L p F^{-/}$versus $L p f^{f / / f l}$ (top panel) and $i L p F^{-/}+$hCETP vs. Lpfl/f + hCETP (bottom panel) (E) Total number of Proteins changed including the number of downregulated (green) and upregulated $(\mathrm{red})$ proteins $(\mathrm{F})$ Main regulated proteins are part of plasma lipoprotein $(\mathrm{G})$ Proteins that significantly change in $i L p F^{--}$versus $L p f^{f / f}$ mice due to hCETP expression. Proteomics normalized to spiked apoA1; complete list of proteins can be found in Supplemental Table II-V. $\mathrm{N}=4-24$ (Proteomics: $L p f^{f / / f l} \mathrm{n}=8, i L p \mathrm{~F}^{-/} \mathrm{n}=8, L p f^{f / f}+\mathrm{hCETP} \mathrm{n}=17, i L p \mathrm{~F}^{-/}+\mathrm{hCETP} \mathrm{n}=10$ ), ${ }^{*} \mathrm{P}<0.05$, ${ }^{* *} \mathrm{P}<0.01,{ }^{* * *} \mathrm{P}<0.001,{ }^{* * *} \mathrm{P}<0.0001,1$-way ANOVA with Tukey's multiple comparison test

\section{Subjects with HyperTG also show decreased HDL-P number, changes in the HDL proteome, and no impairment in total CEC}

To extend our mouse data to humans, we measured HDL-P and CEC in HyperTG patients of which three were diagnosed with LpL deficiency (full characteristics are shown in Supplemental Table VI). Average plasma TG levels were $\sim 750 \mathrm{mg} / \mathrm{dL}$ and total cholesterol levels were $\sim 380 \mathrm{mg} / \mathrm{dL}$ (Figure $6 \mathrm{~A}$ ). HyperTG led to a reduced number of HDL-P (Figure 6B, $P<0.0001$ ), which was mainly due to reduction in medium and large HDL-P, but not small HDL-P. This differs from the mouse data showing a 
reduction in predominantly small HDL-P, but not large HDL-P, and is likely due to species differences in HDL metabolism(e.g. $\left.{ }^{51}\right)$. As in the humanized mouse data (+hCETP, Figure 5B-C), total CEC did not change, meaning that CEC per HDL-P was significantly increased $(P<0.01)$, suggesting an improvement in HDL functionality. This increase was primarily due to an increase in the ABCA1-mediated efflux pathway (Figure 6C-D).

We next assessed HDL proteomics in the human samples. HDL proteomic analysis detected a total of 90 proteins (Figure 6E, Supplemental Table VII). Similar to our humanized mouse data, we found changes in HDL-associated apolipoproteins and PLTP (Figure 6F). Notably, the results of LpL deficient patients are similar to those of HyperTG patients. Taking the human and mouse results together, HyperTG leads to reduction in HDL-P without the creation of intrinsically dysfunctional HDL measured as CEC, while proteomic data mainly reflect the observed changes in HDL associated with LpL deficiency/HyperTG, such as reduced ApoA-I.
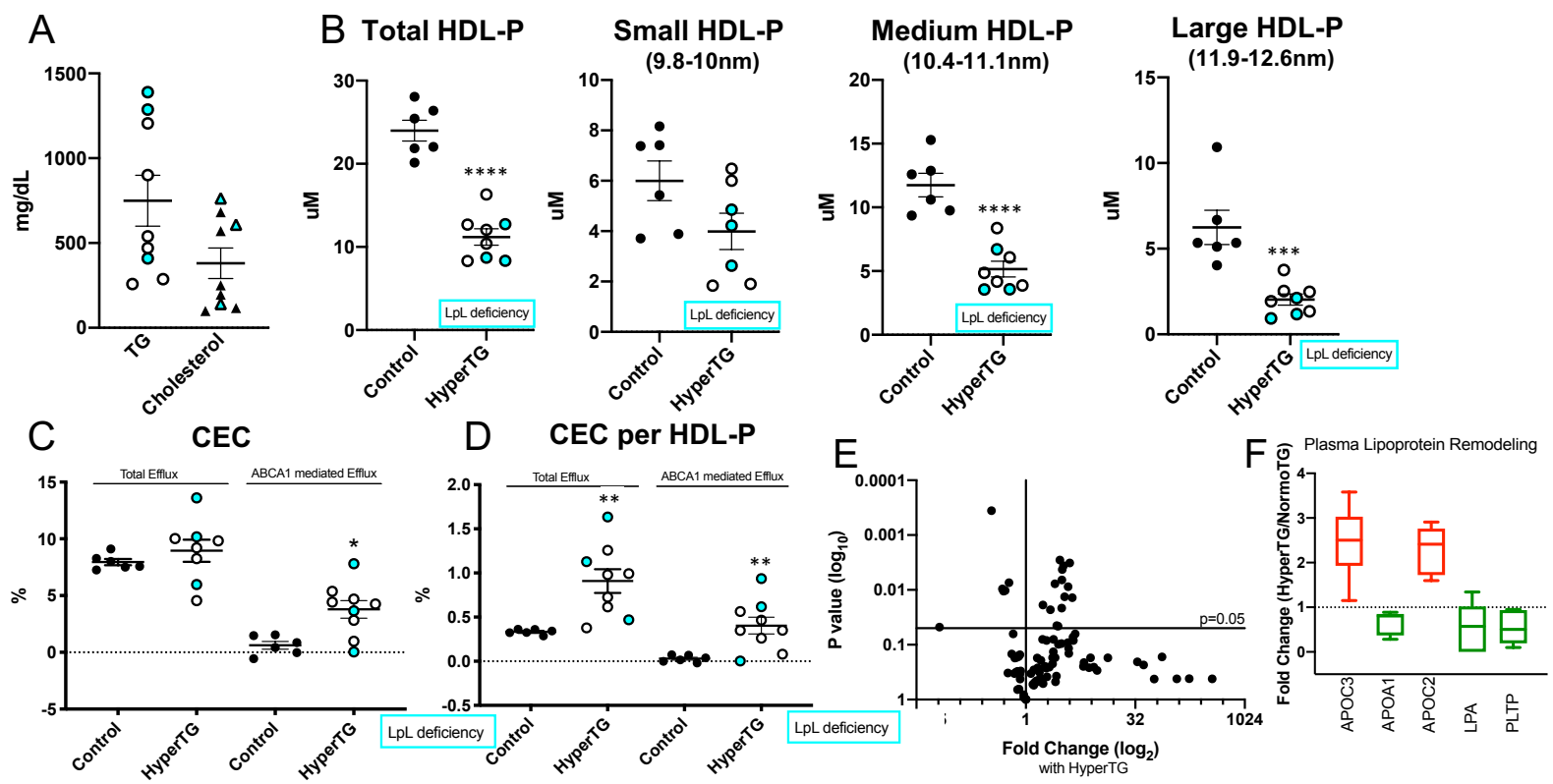

Figure 6. Subjects with HyperTG, incl. LpL deficient subjects show decreased HDL-P, but no impairment in CEC.

(A) Plasma TG and Cholesterol levels (mg/dL). (B) total HDL-P and HDL-P subsets. (C) Total and ABCA1-mediated CEC (\%). (D) CEC per HDL-P (E) Volcanoplot of HDL Proteomics showing changes with HyperTG. (F) Main regulated proteins are part of plasma lipoprotein remodeling pathway. White-filled fots refer to HyperTG patients, blue filled dots refer to L $\mathrm{pL}$ deficient patients. Proteomics normalized to spiked ApoA-I; complete list of proteins can be found in Supplemental 
Table VI. N=6 (Control), N=9 (HyperTG), ${ }^{*} \mathrm{P}<0.05,{ }^{* *} \mathrm{P}<0.01,{ }^{* * * *} \mathrm{P}<0.0001$, students unpaired t-test; (C, D) Mann-Whitney test

\section{Discussion}

Our study shows that despite reductions in HDL-C and HDL-P, LpL deficiencymediated HyperTG did not affect atherosclerosis resolution as assessed by the macrophage content in plaques in aortic arches, roots and BCAs. Furthermore, LpL-deficiency did not affect macrophage inflammation (as judged by M1/M2 phenotypes) or plaque stability judged indirectly by composition (necrotic core, collagen and fibrous cap) in aortic arches in mice. These results are surprising, as HDL-C and HDL-P have been shown to be inversely associated with CVD (reviewed in $^{2}$ ). However, despite a reduction in HDL-C, HDL-P, and CEC, total CEC per particle did not change in HyperTG mice, and was increased in HyperTG humans. Thus, HDL function (CEC) was maintained in the presence of severe HyperTG. This suggests that the inverse association of HDL-C and CVD is not necessarily due to changes in CEC.

Because mice do not have CETP, the HDL-C and HDL-P reductions were likely due to reduced LpL-mediated lipid and protein transfer from TG-rich lipoproteins to HDL. ${ }^{52}$ LpL deficiency led to a change in the HDL-P size distribution with reduced levels of small- and medium-sized particles. In the mouse the combination of reduced lipolysis generated HDL components along with more rapid loss in the kidney might have led to this reduction in smaller HDL-P. With expression of CETP, resulting in TG-rich HDL, HDL-C and HDL-P were further reduced. Along with the reduction in HDL-C and HDL-P, ApoA-I was significantly reduced in mice and humans with HyperTG. Despite marked reductions in $\mathrm{HDL}$, atherosclerosis regression proceeded normally. The reduction in lesional macrophage content was similar in the HyperTG/low HDL and control mice. Thus, despite increased circulating TG levels and marked reductions in HDL-C, atherosclerosis regression proceeded normally and is evidence that LDL-C reduction overrode changes in macrophage $\mathrm{LpL}$ expression and $\mathrm{HDL}$, as discussed below.

Recent clinical data have implicated HDL function rather than HDL-C as a marker for CVD risk. ${ }^{15-17}$ Unsuccessful lowering of CVD mortality in HDL-raising trials using CETP 
inhibition have been explained as a failure to increase reverse cholesterol transport, and perhaps CEC. ${ }^{13}$ Although HyperTG mouse plasma displayed, albeit limited, lower CEC, this reduction was less than what would be expected due to reduced HDL-P. In fact, when corrected for particle number, there was no indication that HDL was dysfunctional. HyperTG-associated reduction in CEC was lost with hCETP expression, as CEC actually increased per HDL-P. Therefore, HyperTG did not reduce HDL function and also did not affect atherosclerosis regression in mice.

In humans, HyperTG is inversely correlated with HDL-C ${ }^{1}$. Therefore, HyperTG was expected to lead to reduced CEC. In agreement with our mouse data, however, HyperTG increased CEC per HDL-P in our human samples. These data confirm that despite the reduction in HDL-P, HDL is still functional in the HyperTG environment. Together, our results indicate that HDL enrichment with TG over cholesterol does not affect function.

In contrast to these results, increasing HDL-P in mice by transgenic overexpression of ApoA-I improved atherosclerosis regression ${ }^{6,9}$. This raises the question why we do not detect impaired regression when HDL-P and ApoA-I are reduced. Perhaps ApoA-I has some function that is exclusive of its actions on HDL to affect CEC. HDL likely has multiple actions in vivo, such as reducing inflammation ${ }^{53}$ and blood coagulation ${ }^{54}$. These CEC-independent functions might be improved by marked increases in HDL. Such other actions of HDL rather than its effects on CEC would be consistent with its relationship to CVD and in agreement with clinical data showing similar overall cholesterol balances of humans with high and low HDL-C levels. ${ }^{18}$ Alternatively, the level of total CEC in the mice with fewer HDL-P particles may have still been sufficient to prevent a greater accumulation of cholesterol in plaque macrophages. For example, in $a p o E^{-/-}$mice we have previously shown that the naturally low level of HDL-P in that model was sufficient to remove the excess free cholesterol from plaques when ACAT was inhibited. ${ }^{55}$

It should also be considered that the effects on regression by changes in HDL-P were likely attenuated by the preservation of ABCA1-mediated cholesterol efflux that we observed. As Rothblat and colleagues have shown ${ }^{56}$, as macrophages get 
progressively loaded by cholesterol, akin to their state in the baseline atherosclerotic plaques, the majority of efflux shifts from the aqueous diffusion pathway to being mediated by ABCA1. Finally, we should also note that despite the decrease in the ABCA1-meditor ApoA-I, other mediators not captured by our HDL measurements could have contributed to preservation of ABCA1-mediated efflux. These could be, among others, pre- $\beta \mathrm{HDL}^{57}$, non-HDL ApoA-IV ${ }^{58}$, or plasminogen ${ }^{59}$.

Part of HyperTG's atherogenicity has been postulated to depend on LpL-mediated lipolysis and the creation of atherogenic remnant particles. Mutations in the LpL gene and heterozygous LpL deficiency, resulting in elevated TG levels, associate with increased CVD risk (OR 1.51, 95\% Cl: 1.39-1.64 per 1-SD increase in TG). ${ }^{60}$ Homozygous LpL-deficient patients have been viewed as atherosclerosis resistant due to the defect on atherogenic remnant production despite their TG reaching levels $>1000 \mathrm{mg} / \mathrm{dL}$. However, more recently, a number of $\mathrm{LpL}$ deficient patients with atherosclerotic CVD have been described. ${ }^{61,62}$ Because the risk of CVD with complete LpL deficiency is unclear our animal data are especially germane. Our global LpL-deficient mice had mild-to-moderate plasma TG levels (approx. 500mg/dL). This level, while lower than many LpL-deficient patients, is not dissimilar from that found in LpL-deficient patients on a very restricted diet that might be comparable to a mouse chow diet. However, on a high fat diet, TG levels of $i L \mathrm{p}^{-/-}$mice increased to $>1,000$ $\mathrm{mg} / \mathrm{dL}$. Thus, the failure to detect differences in regression in these mice is consistent with the hypothesis that nascent TG-rich lipoproteins are not atherogenic.

Regulation of lipolysis and generation of local lipolysis products depends both on LpL activity and circulating TG levels, which provide substrate for the reaction. For this reason, it has been postulated that the combination of partial LpL loss and HyperTG causes high concentrations of liberated toxic lipids or the generation of more atherogenic remnant lipoproteins. Therefore, we also created mice expressing additional LpL in arteries using the Tie2 promoter, which primarily expresses in endothelial cells. Because this transgene was bred onto the LpL knockout background, greater lipolysis along the arterial wall would be expected. This transgene also did not affect atherosclerosis regression. Therefore, neither circulating nascent TG-rich 
lipoproteins nor increasing the capacity of their hydrolysis along the artery wall prevented vascular repair (i.e., regression), in the setting of a marked reduction in LDL- C.

In addition to the points discussed already, our mouse model of LpL deficiency leads to several changes exclusive of circulating HDL-C and TG that should be considered. Macrophages are the only white blood cells that express LpL and are the major source of $L p L$ in atherosclerotic plaques. Whether $L p L$ in atherosclerotic plaques is pro- or anti-atherogenic is still debated. In vitro, macrophage LpL appears to have atherogenic functions, because it creates atherogenic remnant lipoproteins, which are rapidly internalized by macrophages. ${ }^{63}$ Macrophage-specific LpL knockout in vivo reduced atherosclerosis ${ }^{39,40}$, indicating a role of $\mathrm{LpL}$ produced by these cells which is exclusive of changes in circulating lipoproteins.

In contrast to these presumed inflammatory effects of macrophage LpL, fatty acids provided via lipolysis were reported to activate PPAR ${ }^{64}$ and convert macrophages to a less inflammatory phenotype. ${ }^{41,65,66}$ Our recent published data ${ }^{20}$, as well as the present study, however, showed that the transcriptomic profile of regressing atherosclerotic macrophages did not differ between macrophages expressing LpL and LpL-deficient macrophages. Since macrophage LpL has been implicated in in foam cell formation ${ }^{39-41}$, loss of macrophage LpL could have opposite and counterbalancing effects on atherogenesis. To exclude the possibility that we protected our mouse model from possible atherogenic effects of HyperTG, we created HyperTG mice with macrophages expressing LpL. Again, we observed intact atherosclerosis regression. Therefore, macrophage-LpL-mediated lipolysis in atherosclerotic plaques does not impede in HyperTG mice the resolution of atherosclerotic lesions.

In conclusion, our study shows that a reduction in HDL-C and HDL-P associated with LpL-deficiency and CETP expression does not affect atherosclerosis regression. Furthermore, we show that the lipoprotein alterations caused by these interventions, including the TG-enrichment of HDL, does not affect CEC per particle. As already noted, perhaps the lack of effects on atherosclerosis is explained by the continued level of sufficient HDL function, especially through the ABCA1 pathway. Furthermore, 
our data do not support a toxic effect of TG-rich lipoproteins on regression. We expect that these data reflect the human situation in which patients on high doses of LDL-lowering medications show clinical benefit, regardless of their circulating TG levels.

\section{Acknowledgements}

The Authors would like to acknowledge Dr. Joseph Witztum for helpful discussions and advice and thank Dr. Daniel J. Rader for the approval to use the AAV CETP.

\section{Source of Funding}

Funding was provided by American Heart Association (Predoctoral Fellowship 18PRE33990436, TJ), and the NIH (P01 HL092969, EAF, IJG, JWH, KB; P01 HL131481, RO1 HL045095, RO1 HL073029, R01 HL084312, R01 HL129433, EAF, IJG).

\section{Conflicts of Interest}

None. 


\section{References}

1. Dron JS and Hegele RA. Genetics of Triglycerides and the Risk of Atherosclerosis. Curr Atheroscler Rep. 2017;19:31.

2. Ouimet M, Barrett TJ and Fisher EA. HDL and Reverse Cholesterol Transport. Circ Res. 2019;124:1505-1518.

3. Zilversmit DB. Atherogenesis: a postprandial phenomenon. Circulation. 1979;60:473-85.

4. Ebenbichler CF, Kirchmair R, Egger C and Patsch JR. Postprandial state and atherosclerosis. Curr Opin Lipidol. 1995;6:286-90.

5. Feig JE, Rong JX, Shamir R, Sanson M, Vengrenyuk Y, Liu J, Rayner K, Moore K, Garabedian M and Fisher EA. HDL promotes rapid atherosclerosis regression in mice and alters inflammatory properties of plaque monocyte-derived cells. Proc Natl Acad Sci US A. 2011;108:7166-71.

6. Hewing B, Parathath S, Barrett T, Chung WK, Astudillo YM, Hamada T, Ramkhelawon B, Tallant TC, Yusufishaq MS, Didonato JA, Huang Y, Buffa J, Berisha SZ, Smith JD, Hazen SL and Fisher EA. Effects of native and myeloperoxidase-modified apolipoprotein a-I on reverse cholesterol transport and atherosclerosis in mice. Arterioscler Thromb Vasc Biol. 2014;34:779-89.

7. Plump AS, Scott CJ and Breslow JL. Human apolipoprotein A-I gene expression increases high density lipoprotein and suppresses atherosclerosis in the apolipoprotein E-deficient mouse. Proc Natl Acad Sci U S A. 1994;91:9607-11.

8. Rubin EM, Krauss RM, Spangler EA, Verstuyft JG and Clift SM. Inhibition of early atherogenesis in transgenic mice by human apolipoprotein AI. Nature. 1991;353:265-7. 9. Barrett TJ, Distel E, Murphy AJ, Hu J, Garshick MS, Ogando Y, Liu J, Vaisar T, Heinecke JW, Berger JS, Goldberg IJ and Fisher EA. Apolipoprotein AI) Promotes Atherosclerosis Regression in Diabetic Mice by Suppressing Myelopoiesis and Plaque Inflammation. Circulation. 2019;140:1170-1184.

10. Nissen SE, Tsunoda T, Tuzcu EM, Schoenhagen P, Cooper CJ, Yasin M, Eaton GM, Lauer MA, Sheldon WS, Grines CL, Halpern S, Crowe T, Blankenship JC and Kerensky R. Effect of recombinant ApoA-I Milano on coronary atherosclerosis in patients with acute coronary syndromes: a randomized controlled trial. JAMA. 2003;290:2292-300. 11. Shaw JA, Bobik A, Murphy A, Kanellakis P, Blombery P, Mukhamedova N, Woollard K, Lyon S, Sviridov D and Dart AM. Infusion of reconstituted high-density lipoprotein leads to acute changes in human atherosclerotic plaque. Circ Res. 2008;103:1084-91.

12. Group HTC, Landray MJ, Haynes R, Hopewell JC, Parish S, Aung T, Tomson J, Wallendszus K, Craig M, Jiang L, Collins R and Armitage J. Effects of extended-release niacin with laropiprant in high-risk patients. N Engl J Med. 2014;371:203-12.

13. Barter PJ, Brewer HB, Jr., Chapman MJ, Hennekens CH, Rader DJ and Tall AR. Cholesteryl ester transfer protein: a novel target for raising HDL and inhibiting atherosclerosis. Arterioscler Thromb Vasc Biol. 2003;23:160-7.

14. Group HTRC, Bowman L, Hopewell JC, Chen F, Wallendszus K, Stevens W, Collins R, Wiviott SD, Cannon CP, Braunwald E, Sammons E and Landray MJ. Effects of Anacetrapib in Patients with Atherosclerotic Vascular Disease. N Engl J Med. 2017;377:1217-1227. 
15. Rohatgi A, Khera A, Berry JD, Givens EG, Ayers CR, Wedin KE, Neeland IJ, Yuhanna IS, Rader DR, de Lemos JA and Shaul PW. HDL cholesterol efflux capacity and incident cardiovascular events. $N$ Engl J Med. 2014;371:2383-93.

16. Saleheen D, Scott R, Javad S, Zhao W, Rodrigues A, Picataggi A, Lukmanova D, Mucksavage ML, Luben R, Billheimer J, Kastelein JJ, Boekholdt SM, Khaw KT, Wareham N and Rader DJ. Association of HDL cholesterol efflux capacity with incident coronary heart disease events: a prospective case-control study. Lancet Diabetes Endocrinol. 2015;3:507-13.

17. Shea S, Stein JH, Jorgensen NW, McClelland RL, Tascau L, Shrager S, Heinecke JW, Yvan-Charvet L and Tall AR. Cholesterol Mass Efflux Capacity, Incident Cardiovascular Disease, and Progression of Carotid Plaque. Arterioscler Thromb Vasc Biol. 2019;39:8996.

18. Blum CB, Dell RB, Palmer RH, Ramakrishnan R, Seplowitz AH and Goodman DS. Relationship of the parameters of body cholesterol metabolism with plasma levels of HDL cholesterol and the major HDL apoproteins. J Lipid Res. 1985;26:1079-88.

19. Augustus A, Yagyu H, Haemmerle G, Bensadoun A, Vikramadithyan RK, Park SY, Kim JK, Zechner R and Goldberg IJ. Cardiac-specific knock-out of lipoprotein lipase alters plasma lipoprotein triglyceride metabolism and cardiac gene expression. Journal of Biological Chemistry. 2004;279:25050-25057.

20. Chang HR, Josefs T, Scerbo D, Gumaste N, Hu Y, Huggins L-a, Barett T, Chiang S, Grossman J, Bagdasarov S, Fisher EA and Goldberg IJ. Role of LpL ( Lipoprotein Lipase ) in Macrophage Polarization in vitro and in vivo. ATVB. 2019:1-19.

21. Tanigawa H, Billheimer JT, Tohyama J, Zhang Y, Rothblat G and Rader DJ. Expression of cholesteryl ester transfer protein in mice promotes macrophage reverse cholesterol transport. Circulation. 2007;116:1267-73.

22. Reis ED, Li J, Fayad ZA, Rong JX, Hansoty D, Aguinaldo JG, Fallon JT and Fisher EA. Dramatic remodeling of advanced atherosclerotic plaques of the apolipoprotein Edeficient mouse in a novel transplantation model. Journal of vascular surgery. 2001;34:541-7.

23. Chereshnev I, Trogan E, Omerhodzic S, Itskovich V, Aguinaldo JG, Fayad ZA, Fisher EA and Reis ED. Mouse model of heterotopic aortic arch transplantation.J Surg Res. 2003;111:171-6.

24. Trogan E, Fayad ZA, Itskovich VV, Aguinaldo JG, Mani V, Fallon JT, Chereshnev I and Fisher EA. Serial studies of mouse atherosclerosis by in vivo magnetic resonance imaging detect lesion regression after correction of dyslipidemia. Arterioscler Thromb Vasc Biol. 2004;24:1714-9.

25. Rahman K, Vengrenyuk Y, Ramsey SA, Vila NR, Girgis NM, Liu J, Gusarova V, Gromada J, Weinstock A, Moore KJ, Loke P and Fisher EA. Inflammatory Ly6Chi monocytes and their conversion to M2 macrophages drive atherosclerosis regression.J Clin Invest. 2017;127:2904-2915.

26. Josefs T, Barrett TJ, Brown EJ, Quezada A, Wu X, Voisin M, Amengual J and Fisher EA. Neutrophil extracellular traps promote macrophage inflammation and impair atherosclerosis resolution in diabetic mice. JCI Insight. 2020;5.

27. Kanter JE, Kramer F, Barnhart S, Duggan JM, Shimizu-Albergine M, Kothari V, Chait A, Bouman SD, Hamerman JA, Hansen BF, Olsen GS and Bornfeldt KE. A novel strategy to prevent advanced atherosclerosis and lower blood glucose in a mouse model of metabolic syndrome. Diabetes. 2018;67:946-959. 
28. Yuan C, Hu J, Parathath S, Grauer L, Cassella CB, Bagdasarov S, Goldberg IJ, Ramasamy R and Fisher EA. Human Aldose Reductase Expression Prevents Atherosclerosis Regression in Diabetic Mice. Diabetes. 2018;67:1880-1891.

29. Johansson F, Kramer F, Barnhart S, Kanter JE, Vaisar T, Merrill RD, Geng L, Oka K, Chan L, Chait A, Heinecke JW and Bornfeldt KE. Type 1 diabetes promotes disruption of advanced atherosclerotic lesions in LDL receptor-deficient mice. Proc Natl Acad Sci US A. 2008;105:2082-7.

30. Trogan E and Fisher EA. Laser capture microdissection for analysis of macrophage gene expression from atherosclerotic lesions. Methods Mol Biol. 2005;293:221-31.

31. Feig JE and Fisher EA. Laser capture microdissection for analysis of macrophage gene expression from atherosclerotic lesions. Methods Mol Biol. 2013;1027:123-35.

32. Rubinow KB, Vaisar T, Chao JH, Heinecke JW and Page ST. Sex steroids mediate discrete effects on HDL cholesterol efflux capacity and particle concentration in healthy men. J Clin Lipidol. 2018;12:1072-1082.

33. Ronsein GE, Hutchins PM, Isquith D, Vaisar T, Zhao XQ and Heinecke JW. Niacin Therapy Increases High-Density Lipoprotein Particles and Total Cholesterol Efflux Capacity But Not ABCA1-Specific Cholesterol Efflux in Statin-Treated Subjects. Arterioscler Thromb Vasc Biol. 2016;36:404-11.

34. Vaisar T, Kanter JE, Wimberger J, Irwin AD, Gauthier J, Wolfson E, Bahnam V, Wu IH, Shah H, Keenan HA, Greenbaum CJ, King GL, Heinecke JW and Bornfeldt KE. High Concentration of Medium-Sized HDL Particles and Enrichment in HDL Paraoxonase 1 Associate With Protection From Vascular Complications in People With Long-standing Type 1 Diabetes. Diabetes Care. 2020;43:178-186.

35. Hutchins PM, Ronsein GE, Monette JS, Pamir N, Wimberger J, He Y, Anantharamaiah GM, Kim DS, Ranchalis JE, Jarvik GP, Vaisar T and Heinecke JW. Quantification of HDL particle concentration by calibrated ion mobility analysis. Clin Chem. 2014;60:1393-401.

36. Vaisar T, Tang C, Babenko I, Hutchins P, Wimberger J, Suffredini AF and Heinecke JW. Inflammatory remodeling of the HDL proteome impairs cholesterol efflux capacity. $J$ Lipid Res. 2015;56:1519-30.

37. Fabregat A, Jupe S, Matthews L, Sidiropoulos K, Gillespie M, Garapati P, Haw R, Jassal B, Korninger F, May B, Milacic M, Roca CD, Rothfels K, Sevilla C, Shamovsky V, Shorser S, Varusai T, Viteri G, Weiser J, Wu G, Stein L, Hermjakob H and D'Eustachio P. The Reactome Pathway Knowledgebase. Nucleic Acids Res. 2018;46:D649-D655.

38. Parathath S, Grauer L, Huang LS, Sanson M, Distel E, Goldberg IJ and Fisher EA. Diabetes adversely affects macrophages during atherosclerotic plaque regression in mice. Diabetes. 2011;60:1759-69.

39. Babaev VR, Fazio S, Gleaves LA, Carter KJ, Semenkovich CF and Linton MF. Macrophage lipoprotein lipase promotes foam cell formation and atherosclerosis in vivo. J Clin Invest. 1999;103:1697-705.

40. Van Eck M, Zimmermann R, Groot PH, Zechner R and Van Berkel TJ. Role of macrophage-derived lipoprotein lipase in lipoprotein metabolism and atherosclerosis. Arterioscler Thromb Vasc Biol. 2000;20:E53-62.

41. Lindqvist P, Ostlund-Lindqvist AM, Witztum JL, Steinberg D and Little JA. The role of lipoprotein lipase in the metabolism of triglyceride-rich lipoproteins by macrophages. J Biol Chem. 1983;258:9086-92. 
42. Takahashi M, Yagyu H, Tazoe F, Nagashima S, Ohshiro T, Okada K, Osuga J, Goldberg IJ and Ishibashi S. Macrophage lipoprotein lipase modulates the development of atherosclerosis but not adiposity. J Lipid Res. 2013;54:1124-34.

43. Basu D, Hu Y, Huggins LA, Mullick AE, Graham MJ, Wietecha T, Barnhart S, Mogul A, Pfeiffer K, Zirlik A, Fisher EA, Bornfeldt KE, Willecke F and Goldberg IJ. Novel Reversible Model of Atherosclerosis and Regression Using Oligonucleotide Regulation of the LDL Receptor. Circ Res. 2018;122:560-567.

44. Wang L, Gill R, Pedersen TL, Higgins LJ, Newman JW and Rutledge JC.

Triglyceride-rich lipoprotein lipolysis releases neutral and oxidized FFAs that induce endothelial cell inflammation. J Lipid Res. 2009;50:204-13.

45. Zilversmit DB. Role of triglyceride-rich lipoproteins in atherogenesis. Ann $N Y$ Acad Sci. 1976;275:138-44.

46. Goldberg IJ, Eckel RH and McPherson R. Triglycerides and heart disease: still a hypothesis? Arterioscler Thromb Vasc Biol. 2011;31:1716-25.

47. Takahashi M, Hiyama Y, Yokoyama M, Yu S, Hu Y, Melford K, Bensadoun A and Goldberg IJ. In vivo arterial lipoprotein lipase expression augments inflammatory responses and impairs vascular dilatation. Arterioscler Thromb Vasc Biol. 2008;28:45562.

48. Babirak SP, Iverius PH, Fujimoto WY and Brunzell JD. Detection and characterization of the heterozygote state for lipoprotein lipase deficiency. Arteriosclerosis. 1989;9:326-34.

49. Goldberg IJ, Blaner WS, Vanni TM, Moukides M and Ramakrishnan R. Role of lipoprotein lipase in the regulation of high density lipoprotein apolipoprotein metabolism. Studies in normal and lipoprotein lipase-inhibited monkeys. J Clin Invest. 1990;86:463-73.

50. Khera AV, Cuchel M, de la Llera-Moya M, Rodrigues A, Burke MF, Jafri K, French BC, Phillips JA, Mucksavage ML, Wilensky RL, Mohler ER, Rothblat GH and Rader DJ. Cholesterol efflux capacity, high-density lipoprotein function, and atherosclerosis. $N$ Engl J Med. 2011;364:127-35.

51. Walsh A, Ito Y and Breslow JL. High levels of human apolipoprotein A-I in transgenic mice result in increased plasma levels of small high density lipoprotein (HDL) particles comparable to human HDL3. J Biol Chem. 1989;264:6488-94.

52. Bharadwaj KG, Hiyama Y, Hu Y, Huggins LA, Ramakrishnan R, Abumrad NA, Shulman GI, Blaner WS and Goldberg IJ. Chylomicron- and VLDL-derived lipids enter the heart through different pathways: in vivo evidence for receptor- and non-receptormediated fatty acid uptake. J Biol Chem. 2010;285:37976-86.

53. Cockerill GW, Rye KA, Gamble JR, Vadas MA and Barter PJ. High-density lipoproteins inhibit cytokine-induced expression of endothelial cell adhesion molecules. Arterioscler Thromb Vasc Biol. 1995;15:1987-94.

54. Mineo C, Deguchi H, Griffin JH and Shaul PW. Endothelial and antithrombotic actions of HDL. Circ Res. 2006;98:1352-64.

55. Rong JX, Blachford C, Feig JE, Bander I, Mayne J, Kusunoki J, Miller C, Davis M, Wilson M, Dehn S, Thorp E, Tabas I, Taubman MB, Rudel LL and Fisher EA. ACAT inhibition reduces the progression of preexisting, advanced atherosclerotic mouse lesions without plaque or systemic toxicity. Arterioscler Thromb Vasc Biol. 2013;33:4-12. 56. Adorni MP, Zimetti F, Billheimer JT, Wang N, Rader DJ, Phillips MC and Rothblat GH. The roles of different pathways in the release of cholesterol from macrophages. $J$ Lipid Res. 2007;48:2453-62. 
57. Favari E, Lee M, Calabresi L, Franceschini G, Zimetti F, Bernini F and Kovanen PT. Depletion of pre-beta-high density lipoprotein by human chymase impairs ATP-binding cassette transporter A1- but not scavenger receptor class B type I-mediated lipid efflux to high density lipoprotein. J Biol Chem. 2004;279:9930-6.

58. Remaley AT, Stonik JA, Demosky SJ, Neufeld EB, Bocharov AV, Vishnyakova TG, Eggerman TL, Patterson AP, Duverger NJ, Santamarina-Fojo S and Brewer HB, Jr. Apolipoprotein specificity for lipid efflux by the human ABCAI transporter. Biochem Biophys Res Commun. 2001;280:818-23.

59. Pamir N, Hutchins PM, Ronsein GE, Wei H, Tang C, Das R, Vaisar T, Plow E, Schuster V, Koschinsky ML, Reardon CA, Weinberg R, Dichek DA, Marcovina S, Getz GS and Heinecke JW. Plasminogen promotes cholesterol efflux by the ABCA1 pathway. JCI Insight. 2017;2.

60. Khera AV, Won HH, Peloso GM, O'Dushlaine C, Liu D, Stitziel NO, Natarajan P, Nomura A, Emdin CA, Gupta N, Borecki IB, Asselta R, Duga S, Merlini PA, Correa A, Kessler T, Wilson JG, Bown MJ, Hall AS, Braund PS, Carey DJ, Murray MF, Kirchner HL, Leader JB, Lavage DR, Manus JN, Hartzel DN, Samani NJ, Schunkert H, Marrugat J, Elosua R, McPherson R, Farrall M, Watkins H, Lander ES, Rader DJ, Danesh J, Ardissino D, Gabriel S, Willer C, Abecasis GR, Saleheen D, Dewey FE, Kathiresan S, Myocardial Infarction Genetics Consortium DSGCEC and Global Lipids Genetics C. Association of Rare and Common Variation in the Lipoprotein Lipase Gene With Coronary Artery Disease. JAMA. 2017;317:937-946.

61. Benlian P, De Gennes JL, Foubert L, Zhang H, Gagne SE and Hayden M. Premature atherosclerosis in patients with familial chylomicronemia caused by mutations in the lipoprotein lipase gene. $N$ Engl J Med. 1996;335:848-54.

62. Saika Y, Sakai N, Takahashi M, Maruyama T, Kihara S, Ouchi N, Ishigami M, Hiraoka H, Nakamura T, Yamashita S and Matsuzawa Y. Novel LPL mutation (L303F) found in a patient associated with coronary artery disease and severe systemic atherosclerosis. Eur J Clin Invest. 2003;33:216-22.

63. Mead JR, Irvine SA and Ramji DP. Lipoprotein lipase: structure, function, regulation, and role in disease. J Mol Med (Berl). 2002;80:753-69.

64. Orasanu G, Ziouzenkova O, Devchand PR, Nehra V, Hamdy O, Horton ES and Plutzky J. The peroxisome proliferator-activated receptor-gamma agonist pioglitazone represses inflammation in a peroxisome proliferator-activated receptor-alphadependent manner in vitro and in vivo in mice. J Am Coll Cardiol. 2008;52:869-81. 65. Huang SC, Everts B, Ivanova Y, O'Sullivan D, Nascimento M, Smith AM, Beatty W, Love-Gregory L, Lam WY, O'Neill CM, Yan C, Du H, Abumrad NA, Urban JF, Jr., Artyomov MN, Pearce EL and Pearce EJ. Cell-intrinsic lysosomal lipolysis is essential for alternative activation of macrophages. Nature immunology. 2014;15:846-55.

66. Vats D, Mukundan L, Odegaard JI, Zhang L, Smith KL, Morel CR, Wagner RA, Greaves DR, Murray PJ and Chawla A. Oxidative metabolism and PGC-1beta attenuate macrophage-mediated inflammation. Cell metabolism. 2006;4:13-24. 


\section{Supplemental}

A

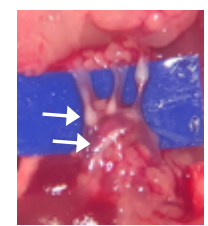

Baseline

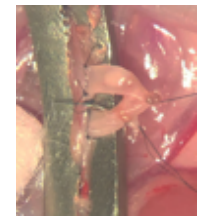

Aortic Transplant
B

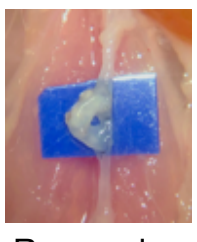

Regression

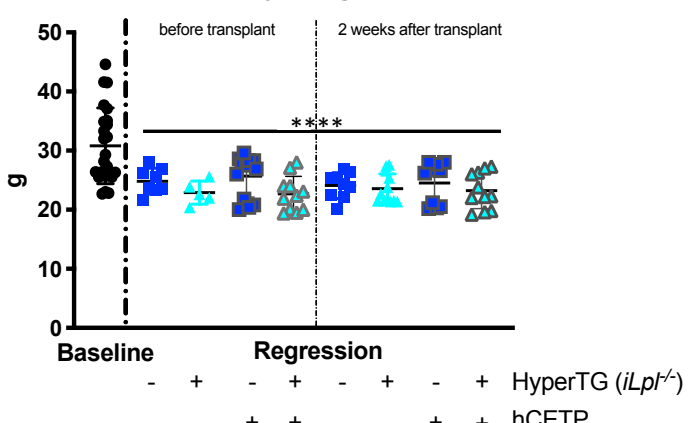

\section{Supplemental Figure I}

(A) Representative pictures of the aortic arch in the donor mouse (left), during aortic transplantation (middle) and after two weeks of atherosclerosis regression (right). Arrows indicate atherosclerotic lesions (B) Body weight in baseline and regression groups before and after the 2 weeks atherosclerosis regression period. $\mathrm{N}=5$-24/group; data represented as mean \pm SEM, ${ }^{* * * *} \mathrm{P}<0.0001,1$-way ANOVA with Tukey‘s multiple comparison test 

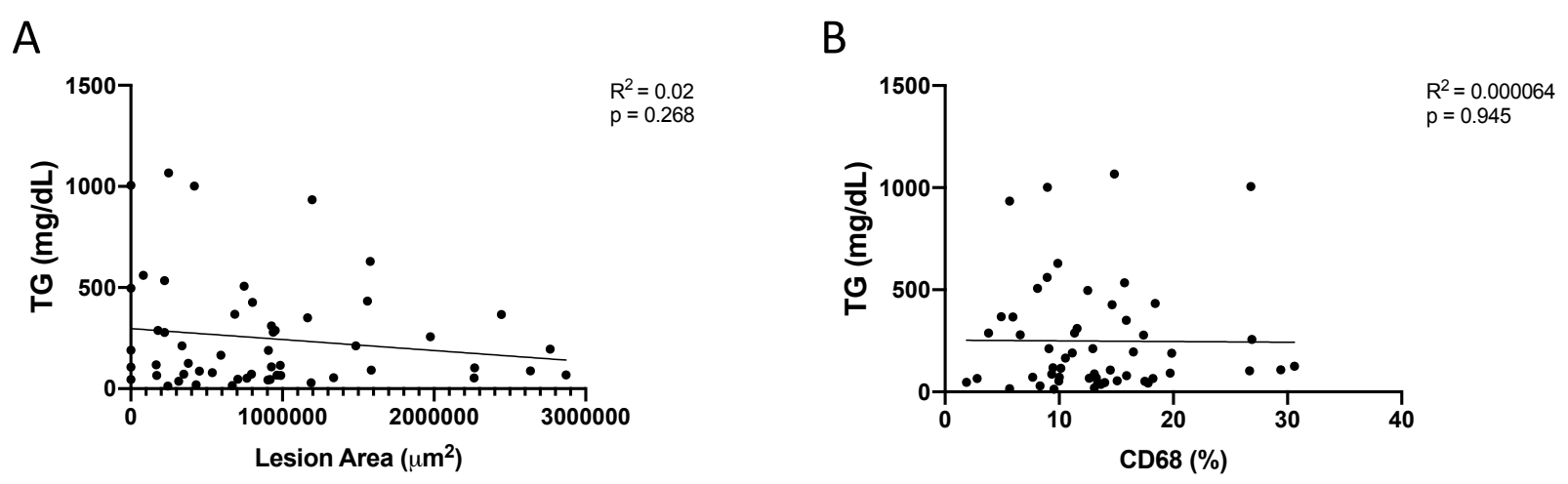

\section{Supplemental Figure II}

Correlation of plasma TG levels with (A) lesion size and (B) \%CD68 
A

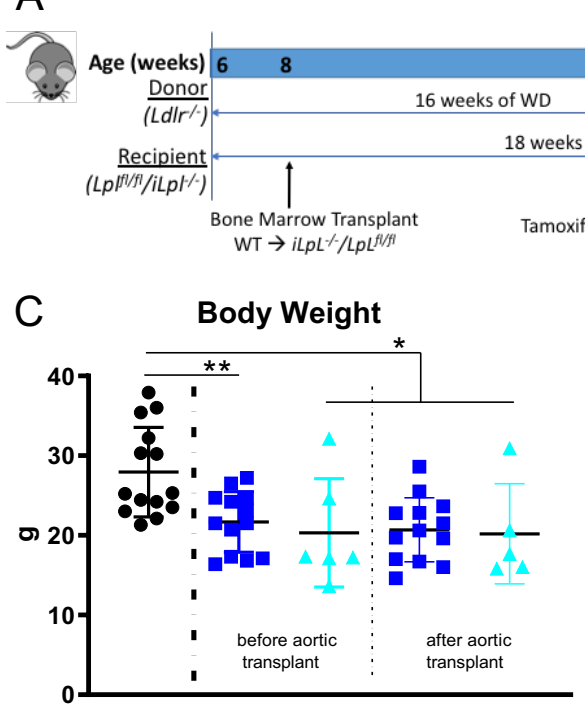

F VLDL-Cholesterol
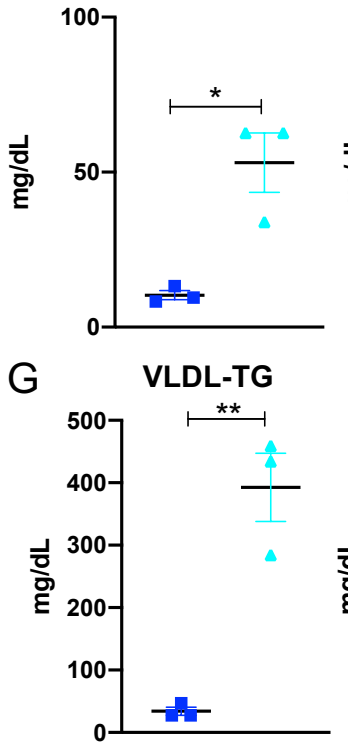

LDL-Cholesterol

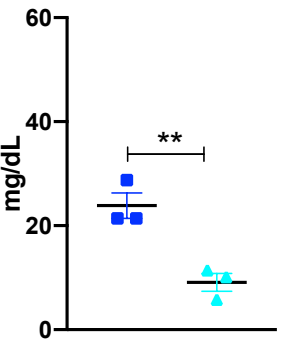

LDL-TG

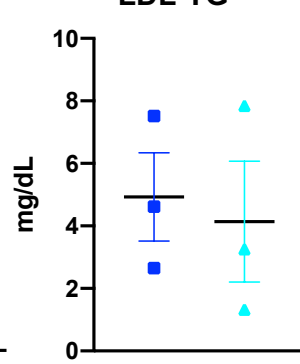

- Baseline $\left(L d l r^{\prime-}\right)$
B

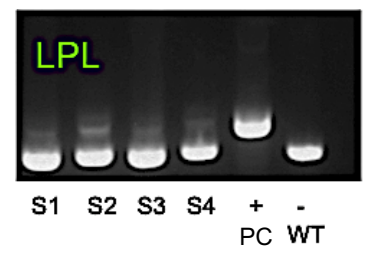

D Total Cholesterol

E Total Triglyceride
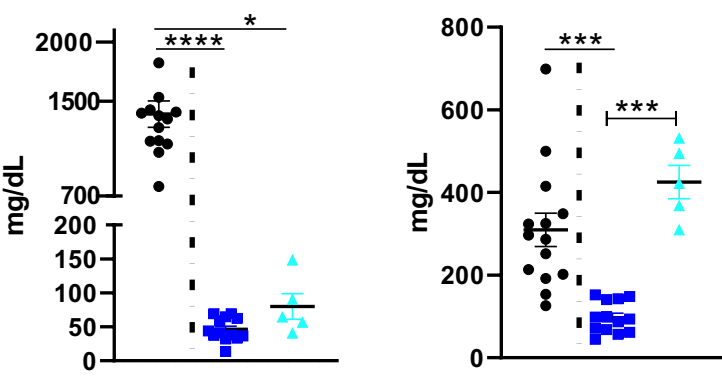

HDL-Cholesterol H
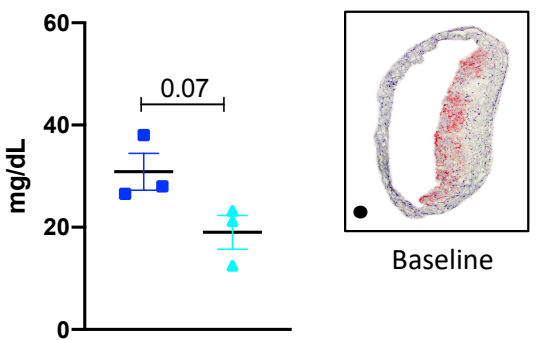

Baseline
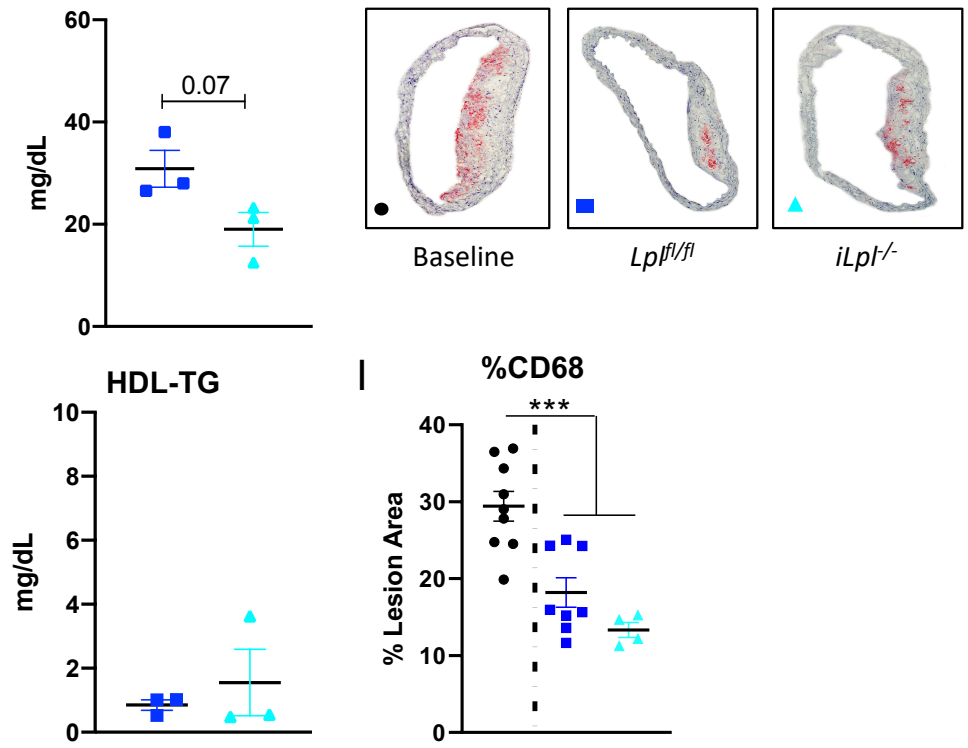

\section{Supplemental Figure III}

$i L p L^{-/}$with $L p L$ expressing macrophages do not impair atherosclerosis regression.

(A) Study setup (B) Genotyping using blood DNA confirming BMT (C) body weight in baseline and regression groups before and after the 2 weeks atherosclerosis regression period (D) Plasma Cholesterol (E) Plasma TG. Cholesterol $(F)$ and triglyceride levels $(G)$ of isolated lipoproteins $(H)$ CD68 staining (I) CD68 area of lesion size (\%). $N=4-14$ /group; data represented as mean \pm SEM, ${ }^{*} \mathrm{P}<0.05,{ }^{* *} \mathrm{P}<0.01,{ }^{* * *} \mathrm{P}<0.001,{ }^{* * *} \mathrm{P}<0.0001,1$-way ANOVA with Tukey's multiple comparison test $(C, I)$, Kruskal-Wallis Test $(D, E)$, students unpaired t-test $(F-G)$. 

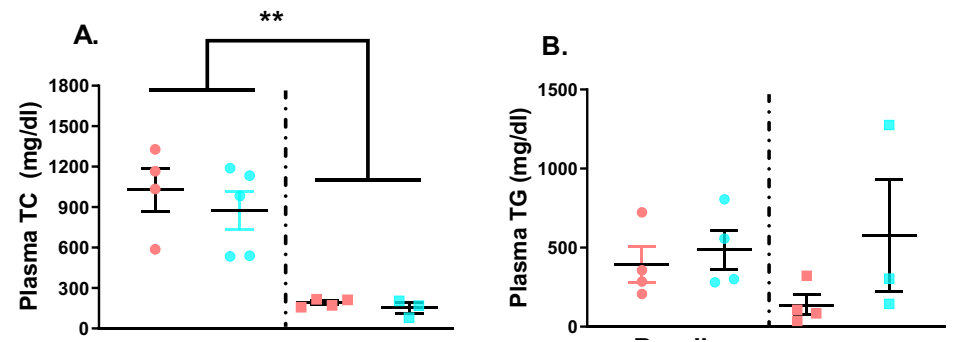

C.

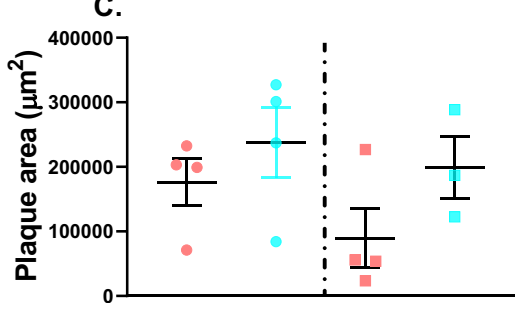

E.

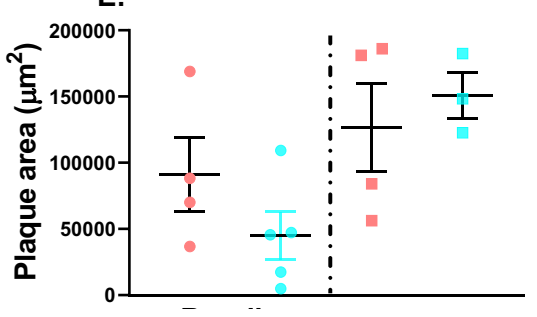

D.
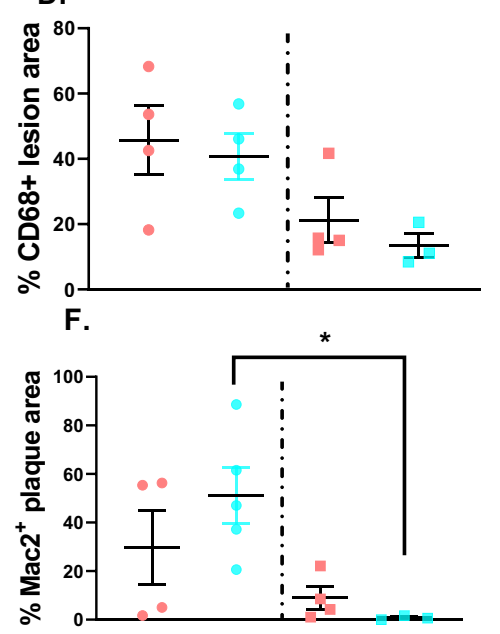

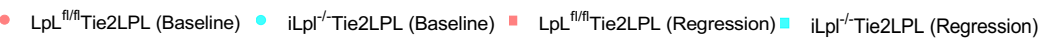

\section{Supplemental Figure IV. Hypertriglyceridemia and enhanced endothelial LPL} expression does not alter atherosclerosis regression in a non-invasive model of regression. Atherosclerosis was created in $L p f^{f / f l}$ Tie2LPL and $i L p{ }^{--}$Tie2LPL mice with LDLR antisense oligonucleotides (ASO) and western diet feeding for 16 weeks. One set of mice were analyzed at 16 weeks as the baseline group and the rest of the mice were treated with SO to induce regression and were analyzed after 3 weeks. The mice in regression group were also treated with tamoxifen at Week 13 to induce hypertriglyceridemia in $\mathrm{iLp}^{-/}$Tie2LPL mice. Plasma (A) total cholesterol (TC) and (B) triglyceride (TG) levels in baseline and regression groups. (C) Total plaque area, (D) \% of macrophages (CD68+) within aortic root lesions, $(E)$ total plaque area, $(F) \%$ of macrophage (Mac2+) in plaques within the $\mathrm{BCA}$ in the baseline and regression groups. $n=3-5$ /group, results expressed as mean \pm SEM. ${ }^{*} \mathrm{P}<0.05$, ${ }^{* *} \mathrm{P}<0.01$ using 1 -way ANOVA with Tukey's multiple comparison test. 
Chapter 4

\section{Supplemental Table I}

List of primer used for qPCR

\begin{tabular}{|c|c|c|}
\hline Gene & Forward (5' - 3') & Reverse (5' - 3') \\
\hline Lpl & AGGTGGACATCGGAGAACTG & TCCCTAGCACAGAAGATGACC3 \\
\hline Plin2 & TCTGCGGCCATGACAAGTG & GCAGGCATAGGTATTGGCAAC \\
\hline Cpt1a & TGCACTACGGAGTCCTGCAA & GGACAACCTCCATGGCTCAG \\
\hline Glut1 & TCGTAACGAGGAGAACCG & GGCCGTGTTGACGATA \\
\hline Fasn & TCTTTCTAACAACCACССТCTGG & CTTCACGACTCCATCACGAATG \\
\hline $\mathrm{Cd} 36$ & ССTTAAAGGAATCCCCGTGT & TGCATTTGCCAATGTCTAGC \\
\hline Tnf & TGGAACTGGCAGAAGAGG & AGACAGAAGAGCGTGGTG \\
\hline Mcp1 & CCCAATGAGTAGGCTGGAGA & TCTGGACCCATTCCTTCTTG \\
\hline Nos2 & CAGCTGGGCTGTACAAACCTT & CATTGGAAGTGAAGCGTTTCG \\
\hline Fizz1 & CCAATCCAGCTAACTATCCСТCC & AAGCCACAAGCACACCCAGT \\
\hline Mrc1 & TGATTACGAGCAGTGGAAGC & GTTCACCGTA-AGCCCAATTT \\
\hline II10 & CTGGACAACATACTGCTAACCG & GGGCATCACTTCTACCAGGTAA \\
\hline
\end{tabular}




\section{Supplemental Table II \\ Overview of detected Proteins}

\begin{tabular}{|c|}
\hline Protein.Gene \\
\hline Actb \\
\hline Agt \\
\hline Ahsg \\
\hline Alb \\
\hline Angpt|3 \\
\hline Antxr2 \\
\hline Apoa2 \\
\hline Apoa4 \\
\hline Apob \\
\hline Apoc1 \\
\hline Apoc2 \\
\hline Apoc3 \\
\hline Apod \\
\hline Apoe \\
\hline Apon \\
\hline Arhgdia \\
\hline $\mathrm{B} 2 \mathrm{~m}$ \\
\hline Blvrb \\
\hline Bpifa2 \\
\hline C3 \\
\hline cab \\
\hline Ca1 \\
\hline $\mathrm{Ca} 2$ \\
\hline Cd97 \\
\hline $\mathrm{Cfl} 1$ \\
\hline $\mathrm{Clu}$ \\
\hline $\mathrm{Cp}$ \\
\hline Ctsd \\
\hline $\mathrm{Dbi}$ \\
\hline Dmkn \\
\hline Dsc3 \\
\hline Dsp \\
\hline Fabp4 \\
\hline Fga \\
\hline $\mathrm{Fgb}$ \\
\hline Gc \\
\hline Gm20425 \\
\hline Gm8909 \\
\hline Gpld1 \\
\hline Gpx3 \\
\hline Gpx5 \\
\hline H2-L \\
\hline H2-Q10 \\
\hline H2-Q4 \\
\hline H2-Q7 \\
\hline Hba \\
\hline Hbbt1 \\
\hline Icam1 \\
\hline Igfals \\
\hline $\mathrm{Ighm}$ \\
\hline IGKC \\
\hline Igkv14-126 \\
\hline Itih4 \\
\hline K1C10_HUMAN \\
\hline K22E_HUMAN \\
\hline Kng1 \\
\hline Krt1 \\
\hline Krt10 \\
\hline Krt13 \\
\hline Krt14 \\
\hline Krt5 \\
\hline Krt72 \\
\hline KRT9 \\
\hline Krt90 \\
\hline Lcat \\
\hline Mb \\
\hline Mug1 \\
\hline Mup18 \\
\hline Mup20 \\
\hline Napsa \\
\hline Pcyox1 \\
\hline Pfn1 \\
\hline Pla2g7 \\
\hline PItp \\
\hline Pm20d1 \\
\hline Pon1 \\
\hline Ppia \\
\hline Ppic \\
\hline Prdx2 \\
\hline Psap \\
\hline Pzp \\
\hline Qsox1 \\
\hline Rbp4 \\
\hline Saa1 \\
\hline Saa2 \\
\hline Saa4 \\
\hline Sell \\
\hline Serpina1a \\
\hline Serpina1b \\
\hline Serpina1e \\
\hline Serpinazk \\
\hline Serpinf2 \\
\hline Tfrc \\
\hline Thbs1 \\
\hline Ttr \\
\hline Vcam1 \\
\hline Vtn \\
\hline
\end{tabular}


Chapter 4

Supplemental Table III

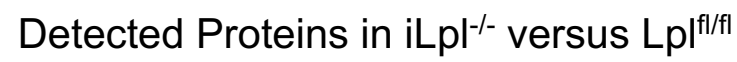
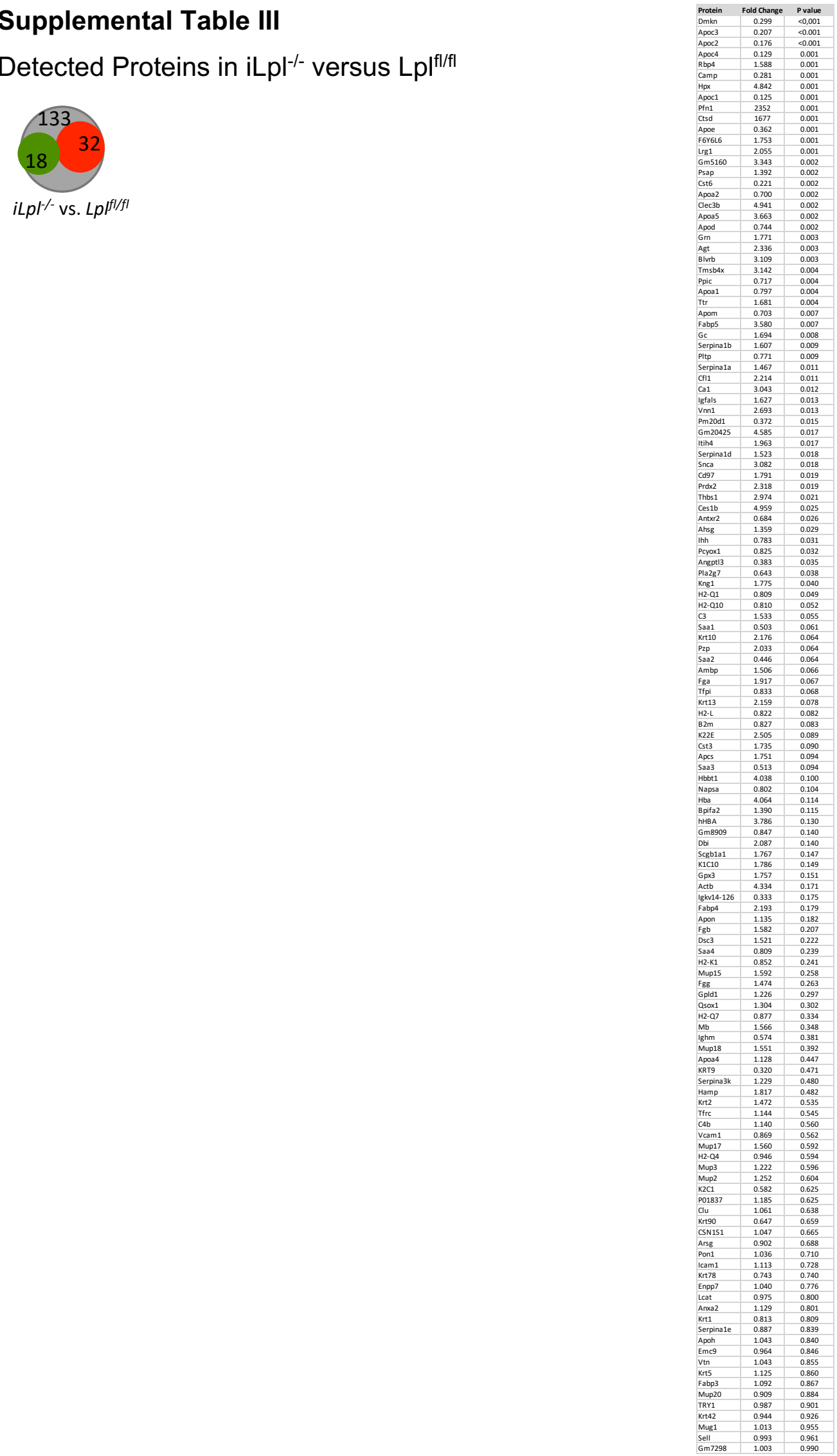


\section{Supplemental Table IV}

Detected Proteins in iL $\mathrm{pl}^{-/-}+\mathrm{hCETP}$ versus Lpl $\mathrm{f}^{\mathrm{fl} / \mathrm{fl}}+\mathrm{hCETP}$

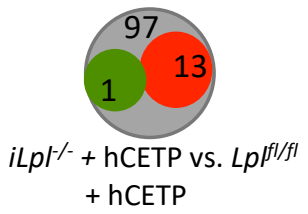

\begin{tabular}{|c|}
\hline Protein \\
\hline Pfn1 \\
\hline $\mathrm{Dbi}$ \\
\hline Arhgdia \\
\hline Fga \\
\hline Dsc3 \\
\hline Hbbt1 \\
\hline Kng1 \\
\hline Blvrb \\
\hline Serpinf2 \\
\hline Prdx2 \\
\hline Fabp4 \\
\hline Ca1 \\
\hline Pzp \\
\hline Apoa2 \\
\hline Itih4 \\
\hline Thbs1 \\
\hline Ppic \\
\hline Cfl1 \\
\hline Serpina3k \\
\hline Serpina1b \\
\hline IGKC \\
\hline Hba \\
\hline Apoa4 \\
\hline Gpld1 \\
\hline Apoa1 \\
\hline Bpifa2 \\
\hline Ppia \\
\hline Ttr \\
\hline Tfrc \\
\hline Qsox1 \\
\hline Igfals \\
\hline Pla2g7 \\
\hline $\mathrm{Fgb}$ \\
\hline Gm20425 \\
\hline Vcam1 \\
\hline Serpina1a \\
\hline $\mathrm{Ca} 2$ \\
\hline Cd97 \\
\hline Pon1 \\
\hline Gpx5 \\
\hline Gpx3 \\
\hline $\mathrm{Mb}$ \\
\hline $\mathrm{Cp}$ \\
\hline C4b \\
\hline Apoc2 \\
\hline Pm20d1 \\
\hline Gc \\
\hline Antxr2 \\
\hline Apoe \\
\hline $\mathrm{B} 2 \mathrm{~m}$ \\
\hline Mup18 \\
\hline Saa2 \\
\hline Mup20 \\
\hline $\mathrm{Ighm}$ \\
\hline Apoc3 \\
\hline Saa1 \\
\hline Agt \\
\hline Lcat \\
\hline Dmkn \\
\hline H2-Q7 \\
\hline KRT9 \\
\hline Napsa \\
\hline Dsp \\
\hline C3 \\
\hline Icam1 \\
\hline Krt14 \\
\hline Serpina1e \\
\hline $\mathrm{Clu}$ \\
\hline Actb \\
\hline H2-L \\
\hline Pltp \\
\hline Apon \\
\hline Ahsg \\
\hline H2-Q4 \\
\hline Igkv14-126 \\
\hline Gm8909 \\
\hline Apod \\
\hline Krt13 \\
\hline Apoc1 \\
\hline Pcyox1 \\
\hline Krt72 \\
\hline Krt5 \\
\hline Mug1 \\
\hline H2-Q10 \\
\hline K1C10_HUMAN \\
\hline Krt90 \\
\hline Krt1 \\
\hline Psap \\
\hline TRYPS \\
\hline Krt10 \\
\hline Angptl3 \\
\hline Rbp4 \\
\hline Saa4 \\
\hline K22E_HUMAN \\
\hline Vtn \\
\hline Sell \\
\hline Ctsd \\
\hline
\end{tabular}




\section{Supplemental Table V}

Overlapping Proteins Supplemental Table II and III

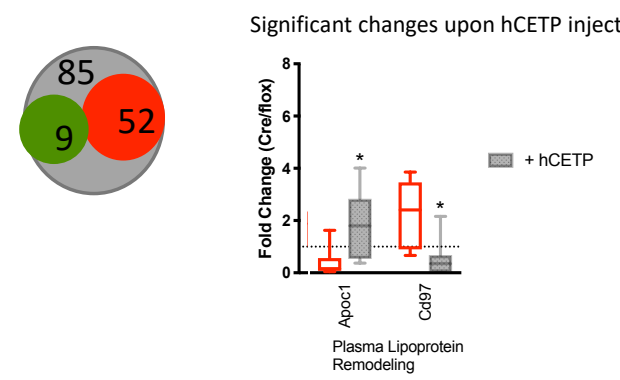

Total of 85 proteins were detected in both proteomic data sets. 9 of them were significantly downregulated, 52 upregulated. They were associated with lipoprotein remodeling.

\begin{tabular}{|c|c|c|c|}
\hline & Cre vs Flox & Cre vs flox hCETP & \\
\hline Protein & Fold Change & Fold Change & Pvalue \\
\hline Qsox1 & 1.304 & 0.172 & 0.001 \\
\hline Cd97 & 1.791 & 0.168 & 0.002 \\
\hline Apoc1 & 0.125 & 1.110 & 0.019 \\
\hline Thbs1 & 2.974 & 3.245 & 0.032 \\
\hline Ppic & 0.717 & 1.414 & 0.047 \\
\hline Apoc3 & 0.206 & 0.806 & 0.058 \\
\hline Tfrc & 1.143 & 0.176 & 0.064 \\
\hline H2-L & 0.821 & 1.178 & 0.088 \\
\hline Serpina3k & 1.228 & 2.132 & 0.121 \\
\hline Apon & 1.135 & 1.284 & 0.149 \\
\hline Pltp & 0.771 & 1.544 & 0.163 \\
\hline Serpina1a & 1.466 & 1.468 & 0.183 \\
\hline Actb & 4.334 & 0.223 & 0.188 \\
\hline Dmkn & 0.298 & 0.713 & 0.195 \\
\hline $\mathrm{Fgb}$ & 1.581 & 1.616 & 0.197 \\
\hline H2-Q10 & 0.810 & 1.102 & 0.198 \\
\hline $\mathrm{H} 2-\mathrm{Q} 4$ & 0.946 & 0.899 & 0.200 \\
\hline Gm8909 & 0.847 & 1.164 & 0.210 \\
\hline Apoc2 & 0.176 & 0.669 & 0.211 \\
\hline Rbp4 & 1.587 & 1.128 & 0.214 \\
\hline Ahsg & 1.358 & 1.192 & 0.215 \\
\hline Sell & 0.992 & 0,952 & 0.223 \\
\hline Apod & 0.744 & 0.849 & 0.238 \\
\hline Krt5 & 1.124 & 0.602 & 0.238 \\
\hline Gc & 1.693 & 1.459 & 0.252 \\
\hline Saa2 & 0.446 & 1.634 & 0.253 \\
\hline Mup20 & 0.909 & 1.812 & 0.256 \\
\hline Cfl1 & 2.214 & 2.861 & 0.274 \\
\hline Bpifa2 & 1.389 & 0.324 & 0.286 \\
\hline $\mathrm{Dbi}$ & 2.087 & 1.925 & 0.295 \\
\hline Apoa4 & 1.128 & 1.383 & 0.300 \\
\hline Krt10 & 2.175 & 0.826 & 0.303 \\
\hline Saa1 & 0.503 & 1.438 & 0.304 \\
\hline $\mathrm{Mb}$ & 1.566 & 1.692 & 0.305 \\
\hline Pfn1 & 2.352 & 2.095 & 0.305 \\
\hline Apoe & 0.362 & 1.352 & 0.326 \\
\hline B2m & 0.827 & 1.214 & 0.328 \\
\hline Ca1 & 3.042 & 2.462 & 0.328 \\
\hline KRT9 & 0.320 & 1.306 & 0.330 \\
\hline Mug1 & 1.012 & 1.226 & 0.334 \\
\hline $\mathrm{H} 2-\mathrm{Q} 7$ & 0.876 & 1.388 & 0.338 \\
\hline Serpina1e & 0.887 & 1.253 & 0.352 \\
\hline Vtn & 1.043 & 0.939 & 0.359 \\
\hline Napsa & 0.801 & 0.859 & 0.368 \\
\hline Pzp & 2.033 & 4.668 & 0.383 \\
\hline Krt1 & 0.813 & 0.829 & 0.397 \\
\hline Dsc3 & 1.521 & 2.094 & 0.401 \\
\hline $\mathrm{Hba}$ & 4.063 & 2.407 & 0.405 \\
\hline Fga & 1.916 & 1.907 & 0.415 \\
\hline Serpina $1 b$ & 1.607 & 1.587 & 0.426 \\
\hline Gpld1 & 1.226 & 0.583 & 0.434 \\
\hline Psap & 1.392 & 0.759 & 0.437 \\
\hline Krt90 & 0.646 & 0.822 & 0.449 \\
\hline Agt & 2.336 & 1.204 & 0.454 \\
\hline Antxr2 & 0.684 & 0.494 & 0.456 \\
\hline Blvrb & 3.108 & 2.448 & 0.463 \\
\hline C3 & 1.533 & 0.805 & 0.466 \\
\hline Igfals & 1.626 & 0.124 & 0.469 \\
\hline Ctsd & 1.677 & 1.025 & 0.472 \\
\hline Gm20425 & 4.584 & 2.947 & 0.486 \\
\hline Kng1 & 1.774 & 3.343 & 0.488 \\
\hline Krt13 & 2.158 & 0.644 & 0.504 \\
\hline Pm20d1 & 0.372 & 2.010 & 0.507 \\
\hline Vcam1 & 0.869 & 0.271 & 0.508 \\
\hline Pcyox1 & 0.824 & 0.908 & 0.508 \\
\hline Fabp4 & 2.192 & 2.208 & 0.515 \\
\hline C4b & 1.140 & 0.554 & 0.526 \\
\hline Apoa2 & 0.700 & 0.818 & 0.553 \\
\hline Saa4 & 0.809 & 1.054 & 0.571 \\
\hline Itih4 & 1.963 & 2.669 & 0.587 \\
\hline Angpt|3 & 0.383 & 1.155 & 0.629 \\
\hline Icam1 & 1.112 & 0.476 & 0.642 \\
\hline Ttr & 1.681 & 1.404 & 0.673 \\
\hline Mup18 & 1.551 & 1.697 & 0.716 \\
\hline $\mathrm{Clu}$ & 1.060 & 0.775 & 0.716 \\
\hline Lcat & 0.974 & 0.792 & 0.752 \\
\hline Gpx3 & 1.756 & 1.580 & 0.783 \\
\hline Prdx2 & 2.318 & 2.220 & 0.802 \\
\hline Hbbt1 & 4.038 & 3.166 & 0.820 \\
\hline Igkv14-126 & 0.333 & 1.357 & 0.854 \\
\hline Pon1 & 1.036 & 0.658 & 0.900 \\
\hline Apoa1 & 0.797 & 0.705 & 0.914 \\
\hline Pla2g7 & 0.643 & 0.288 & 0.925 \\
\hline Ighm & 0.574 & 1.564 & 0.989 \\
\hline
\end{tabular}




\section{Supplemental Table VI}

Patient characteristics

\begin{tabular}{|llc|}
\hline Patient & Diagnosis & LpL deficient \\
& 1 HyperTG, Pancreatitis & \\
& 2 LCAT Deficient & \\
& 3 HyperTG & \\
& 4 HyperTG & $X$ \\
5 Hyperlipidemia & \\
6 HyperTG, Pancreatitis, T1D, Ketoacidosis & $X$ \\
7 No Pancreatitis, Good TGs, On Meds & \\
8 HyperTG & \\
9 Recurring Pancreatitis, DM & \\
\hline
\end{tabular}




\section{Supplemental Table VII}

\section{HyperTG versus Control}

90 detected proteins in human samples, 6 being significantly downregulated, 14 upregulated with HyperTG.

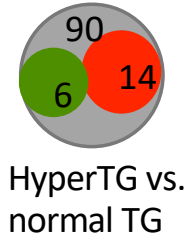

\begin{tabular}{|c|c|c|}
\hline Protein & Fold change & P-value \\
\hline ANTXR2 & 0.336 & $<0.001$ \\
\hline ORM1 & 2.940 & 0.003 \\
\hline GC & 4.023 & 0.003 \\
\hline $\mathrm{FGG}$ & 3.300 & 0.004 \\
\hline FGB & 3.164 & 0.004 \\
\hline SERPINA1 & 3.128 & 0.007 \\
\hline APOA1 & 0.586 & 0.007 \\
\hline APOC2 & 2.509 & 0.008 \\
\hline FGA & 3.798 & 0.009 \\
\hline MENT & 0.476 & 0.010 \\
\hline CD44 & 0.519 & 0.010 \\
\hline PLTP & 0.494 & 0.011 \\
\hline $\mathrm{A} 1 \mathrm{BG}$ & 3.403 & 0.013 \\
\hline SERPINF1 & 4.354 & 0.014 \\
\hline APOH & 1.718 & 0.019 \\
\hline AHSG & 3.104 & 0.021 \\
\hline AZGP1 & 2.171 & 0.023 \\
\hline ALB & 4.670 & 0.039 \\
\hline APOC3 & 2.666 & 0.045 \\
\hline PPBP & 2.769 & 0.046 \\
\hline LPA & 0.065 & 0.048 \\
\hline APOA2 & 0.669 & 0.063 \\
\hline $\mathrm{C} 1 \mathrm{R}$ & 4.764 & 0.064 \\
\hline DEFA1 & 4.460 & 0.073 \\
\hline APOC4 & 2.399 & 0.080 \\
\hline IGHA1 & 1.696 & 0.080 \\
\hline$\pi R$ & 1.927 & 0.083 \\
\hline IGHG1 & 4.492 & 0.084 \\
\hline IGKC & 3.779 & 0.091 \\
\hline PLA2G7 & 3.085 & 0.095 \\
\hline S100A8 & 2.797 & 0.098 \\
\hline CST3 & 3.877 & 0.116 \\
\hline APOE & 2.382 & 0.134 \\
\hline RBP4 & 3.897 & 0.143 \\
\hline FGFBP2 & 0.638 & 0.147 \\
\hline APOD & 0.831 & 0.152 \\
\hline VTN & 0.761 & 0.162 \\
\hline APOM & 0.798 & 0.164 \\
\hline CA1 & 74.468 & 0.166 \\
\hline CLU & 0.745 & 0.171 \\
\hline APOA4 & 2.524 & 0.172 \\
\hline KRT10 & 2.325 & 0.172 \\
\hline APOA5 & 7.754 & 0.173 \\
\hline C4BPA & 13.435 & 0.173 \\
\hline SAA4 & 0.707 & 0.174 \\
\hline APOL1 & 1.436 & 0.175 \\
\hline APMAP & 1.608 & 0.192 \\
\hline CA2 & 34.141 & 0.204 \\
\hline A2M & 6.285 & 0.211 \\
\hline KRT2 & 2.327 & 0.224 \\
\hline SAA1 & 41.561 & 0.234 \\
\hline CAMP & 1.832 & 0.249 \\
\hline AMBP & 6.197 & 0.249 \\
\hline KRT5 & 1.876 & 0.254 \\
\hline KRT1 & 2.102 & 0.256 \\
\hline IGHM & 8.624 & 0.257 \\
\hline B2M & 7.417 & 0.262 \\
\hline LCAT & 1.455 & 0.263 \\
\hline KRT14 & 1.901 & 0.267 \\
\hline APOC1 & 1.288 & 0.288 \\
\hline TF & 9.556 & 0.294 \\
\hline PCYOX1 & 0.847 & 0.301 \\
\hline CD99 & 0.769 & 0.306 \\
\hline MADCAM1 & 0.732 & 0.316 \\
\hline S100A7 & 1.533 & 0.318 \\
\hline $\mathrm{SHH}$ & 0.566 & 0.327 \\
\hline PSAP & 0.697 & 0.335 \\
\hline C3 & 1.170 & 0.335 \\
\hline PON3 & 0.827 & 0.342 \\
\hline IGHG2 & 2.638 & 0.374 \\
\hline KRT9 & 1.953 & 0.384 \\
\hline HBA1 & 175.992 & 0.415 \\
\hline HBB & 365.166 & 0.417 \\
\hline HBD & 119.098 & 0.420 \\
\hline PON1 & 0.832 & 0.421 \\
\hline HBZ & 58.339 & 0.422 \\
\hline ANXA2P2 & 1.663 & 0.423 \\
\hline KRT75 & 1.534 & 0.428 \\
\hline HP & 1.392 & 0.456 \\
\hline KRT6A & 1.532 & 0.470 \\
\hline HLA-A & 2.551 & 0.473 \\
\hline DCD & 1.321 & 0.486 \\
\hline KRT16 & 1.724 & 0.510 \\
\hline C4A & 1.251 & 0.528 \\
\hline HPR & 1.282 & 0.549 \\
\hline LTF & 0.806 & 0.643 \\
\hline SFTPB & 0.768 & 0.657 \\
\hline POTEF & 0.934 & 0.818 \\
\hline KRT17 & 0.936 & 0.916 \\
\hline PFN1 & 1.011 & 0.986 \\
\hline C3orf85 & 0.998 & 0.996 \\
\hline
\end{tabular}




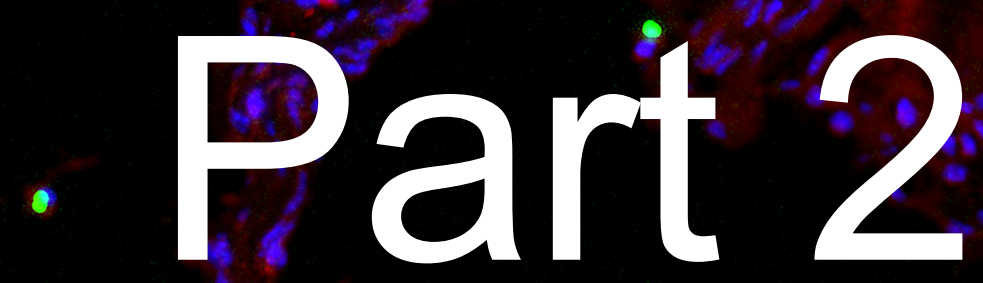

Hyperglycemia

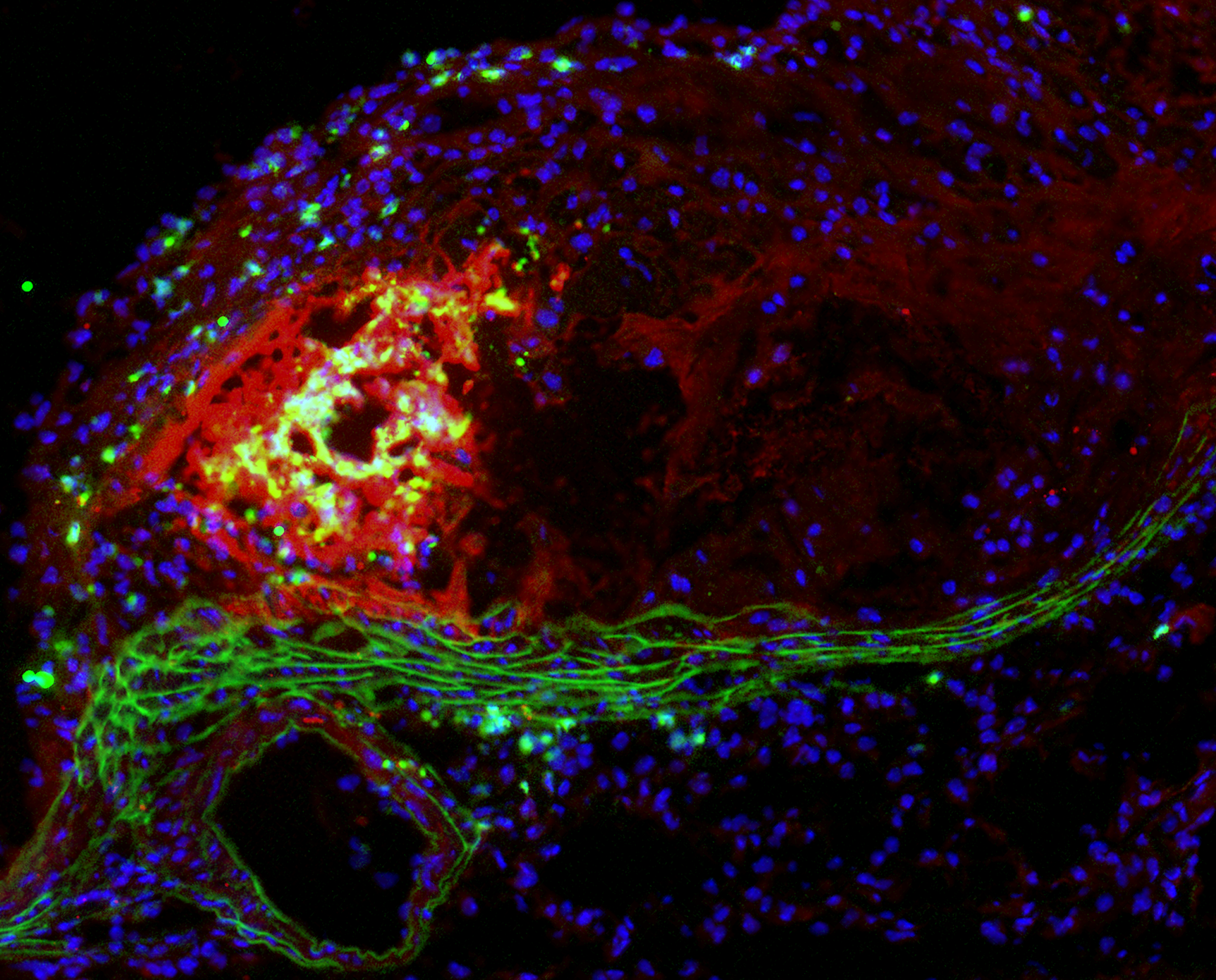




\section{Neutrophil Extracellular Traps (NETs) Promote Macrophage Inflammation and Impair Atherosclerosis Resolution in Diabetic Mice}

Tatjana Josefs* ${ }^{*}$ Tessa J. Barrett*, Emily Brown, Alexandra Quezada, Xiaoyun Wu, Maud Voisin,
Jaume Amengual, Edward A. Fisher

* Equal contribution

JCI Insight. 2020;5(7):e134796 


\section{Abstract}

Neutrophil extracellular traps (NETs) promote inflammation and atherosclerosis progression. NETs are increased in diabetes and impair the resolution of inflammation during wound healing. Atherosclerosis resolution, a process resembling wound healing, is also impaired in diabetes. Thus, we hypothesized that NETs impede atherosclerosis resolution in diabetes by increasing plaque inflammation. Indeed, transcriptomic profiling of plaque macrophages from NET positive and negative areas in low-density lipoprotein receptor-deficient $\left(L d / r^{\prime-}\right)$ mice revealed inflammasome and glycolysis pathway upregulation, indicating a heightened inflammatory phenotype. We found that NETs decline during atherosclerosis resolution, which was induced by reducing hyperlipidemia in non-diabetic mice, but they persist in diabetes, exacerbating macrophage inflammation and impairing resolution. In diabetic mice deoxyribonuclease 1 (DNase1) treatment reduced plaque NETs content and macrophage inflammation, promoting atherosclerosis resolution after lipid-lowering. Given that humans with diabetes also exhibit impaired atherosclerosis resolution with lipid-lowering, these data suggest that NETs contribute to the increased cardiovascular disease risk in this population and are a potential therapeutic target. 


\section{Introduction}

Cardiovascular disease (CVD) and diabetes are both associated with leukocytosis (1, 2). We and others have previously reported that hyperglycemia in mice impaired the resolution of atherosclerosis after lipid-lowering $(3,4)$. Hyperglycemia also stimulated myelopoiesis in the mice, which increased circulating levels of monocytes and neutrophils and their entry into plaques (5-7). While the contribution of monocytederived macrophages to atherogenesis has been extensively studied, less is known about neutrophils. Accumulating evidence indicates that neutrophils are also important protagonists in plaque progression (8). Their ability to form neutrophil extracellular traps (NETs) (9), structures found in human and mouse plaques (10-12), is of increasing interest in the context of atherogenesis due to the proinflammatory nature of NETs (13).

First reported in 2004 (14), NETosis is a process in which, in response to pathogens and sterile inflammatory stimuli, neutrophils release cytosolic and nuclear material forming a net-like extracellular structure (15). Relevant to atherogenesis, stimulators of NETosis include cholesterol crystals, oxidized low-density lipoprotein, oxysterols, platelets, and various chemokines (9). NETosis is not only stimulated by inflammation but can also promote it (9), for example, by increasing monocyte recruitment to inflamed sites and triggering macrophages to release reactive oxygen species and proinflammatory cytokines (16). The effects of NETs on plaque macrophages are thought to involve activation of their inflammasomes, but direct evidence in vivo to support this is scant (17).

In mice, genetic and pharmacological inhibition of NETosis reduces plaque size, inflammation, and features of instability $(10,18)$. In humans, circulating NET biomarkers positively correlate with atherosclerotic plaque size, and are independently associated with clinical severity of CVD and the incidence of major adverse cardiac events $(19,20)$. One clinical population in which NETs are hypothesized to be particularly atherogenic is those with diabetes. Compared to people without diabetes, those with diabetes exhibit increased CVD risk and impaired atherosclerosis resolution, or plaque regression, after lipid-lowering therapy $(21,22)$. That NETs could be an important basis of these findings is supported by studies in which circulating 
NET biomarkers are correlated with both CVD and diabetes severity (23). Factors promoting NETosis in diabetes include both neutrophilia, and hyperglycemia, which primes neutrophils for NET formation (24) and provides an increased substrate for NETosis, which is glucose dependent (25).

To date, CVD clinical studies have addressed the role of NETs only in the progression of atherosclerosis. Given that most patients, including those with diabetes, commence primary or secondary CVD prevention (typically lipid-lowering) after the formation of plaques, resolution of atherosclerosis is an important, and largely unmet, clinical goal. The information presented above suggested to us that the failure of lipid-lowering therapies to fully reduce CVD risk in patients with diabetes, or to maximally resolve atherosclerosis in mice and patients with diabetes (reviewed in (26-28)), represented adverse consequences of the increased plaque level of NETs in this metabolic setting (25). This phenomenon would be similar to NETs impairing wound healing in diabetes (24).

Thus, we have investigated the effect of NETs on atherosclerosis resolution in mice with and without diabetes, using deoxyribonuclease 1 (DNase1) as a tool to deplete NETs from plaques (as in (10)). NETs had a direct proinflammatory influence on plaque macrophages, while DNase1-mediated depletion was associated with dampening of macrophage inflammation and improved atherosclerosis resolution after lipid-lowering despite ongoing hyperglycemia. Given that DNase1 has approved applications in other human diseases (e.g., cystic fibrosis), the results also suggest a new mechanistic approach to improving atherosclerosis resolution in high-risk patients with diabetes, which remains an important clinical goal.

\section{Materials and Methods}

Animals \& Atherosclerosis Regression Study: Ldlr ${ }^{-/}$mice (Jackson Laboratory) were fed western diet $(0.3 \%$ cholesterol; Dyets Inc. D101977Gi) for 16 weeks (progression), followed by 4-weeks (regression) on chow diet. Streptozotocin (STZ; Sigma Aldrich, cat. S0130-500MG; 0.05mg/g body weight) or sodium citrate (control) was injected daily for 5 days intraperitoneal (i.p.) just prior to regression to induce diabetes, as described previously (7). During the four-week regression period, DNase1 
$(62.5 \mu \mathrm{g} /$ mouse Dornase Alfa, Genentech, Inc.) in $0.9 \% \mathrm{NaCl}$ or $0.9 \% \mathrm{NaCl}$ only (control) was injected i.p. every other day. At the day of sacrifice, mice were anesthetized via intraperitoneal (i.p.) injection of ketamine $(100 \mathrm{mg} / \mathrm{kg}$ ) and xylazine $(10 \mathrm{mg} / \mathrm{kg})$. Blood was collected via cardiac puncture in EDTA-containing tubes and mice perfused with $10 \%$ sucrose in saline solution $(0.9 \% \mathrm{NaCl})$. Collected aortic roots were embedded in optimal cutting temperature (OCT) compound and frozen at $-80^{\circ} \mathrm{C}$. All procedures were conducted under NYU Animal Care and Use Committee approved protocol \#160725 (Creating glucose-responsive cardiovascular complications in the mouse).

Plasma Lipid and Glucose Measurements: Total cholesterol was measured using Total Cholesterol E Kit (Wako Life Science, cat. NC9138103). Plasma glucose levels were determined using the Contour Blood Glucose Monitoring System (Bayer).

Flow Cytometry: Total white blood cell counts were measured from freshly collected mouse blood using a hematology cell counter (Oxford Science Inc.). Red blood cells were lysed with buffer (Sigma-Aldrich), and cells stained with CD45 Pe/Cy7 (BioLegend, Cat. 103114), CD115 PE (BioLegend, Cat. 135505), and APC Ly-6G/Ly-6C (Gr-1, BioLegend, Cat. 108412). Neutrophils were identified by flow cytometry using LSRII analyzer and analyzed using FlowJo v10.

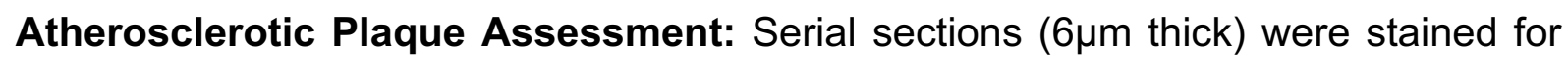
CD68 (rat anti-mouse CD68, Bio-Rad, Cat. MCA1957), as done before (29). Briefly, sections were fixed and permeabilized with $100 \%$ ice-cold acetone, blocked, and stained with a rat anti-mouse CD68 antibody (Bio-Rad, cat. MCA1957) followed by an incubation with a biotinylated rabbit anti-rat $\lg G$ secondary antibody (Vector Laboratories, cat. BA4000), and visualized using the Vectastain ABC kit (Fisher Scientific, Cat. NC9313719). Slides were counterstained with hematoxylin/eosin (Sigma), dehydrated in an ethanol gradient and xylene (Fisher Scientific, NH), and mounted with coverslips using Permount (Fisher Scientific). NETs were identified using colocalization immunofluorescence staining of the lymphocyte antigen 6 complex locus G6D (Ly6G; BD Bioscience, cat. 551459), myeloperoxidase (MPO) (Abcam, cat. ab90812), and citrullinated H3 (H3Cit; Abcam, cat. ab5103). Tissue was fixed in 
formalin, permeabilized using $0.5 \%$ Triton-X, followed by incubation of primary $A b \mathrm{o} / \mathrm{n}$ at $4^{\circ} \mathrm{C}$ and stained with the secondary antibodies for $2 \mathrm{~h}$ (Thermo Fisher Scientific, cat. A21434 \& A21245). The same protocol was used to stain for NLR family pyrin domaincontaining protein 3 (NLRP3; Abcam, cat. ab4207) and Caspase-1 (Millipore, cat. AB1871; secondary antibodies: Thermo Fisher Scientific, cat. A-11055 \& A-31573). Inducible nitric oxide synthase (iNOS) staining was done on formalin-fixed tissue, permeabilized using $0.05 \%$ Tween and $1 \%$ Triton-X, followed by incubation with a conjugated iNOS antibody (Abcam, cat. Ab209027) incubated o/n at $4^{\circ} \mathrm{C}$. Collagen content was determined by Picosirius Red staining as done before (30). NETs and iNOS stainings were imaged using Hamatsu NanoZoomer 2.0; a Leica $\mathrm{SP}_{5}$ Confocal Microscope was used to obtain images of NLRP3 and Caspase1 staining. Collagen was visualized using polarized light microscopy (Zeiss Axio Observer). ImagePro Plus 7.0 software was used to determine plaque, CD68+, collagen and necrotic core areas. $\mathrm{NET}^{+}$area, NLRP3 ${ }^{+}, \mathrm{Caspase}^{+}$, and $\mathrm{NOS}^{+}$cells were determined by Image J 1.51r. These data were then divided by the plaque area of the section to get the \% positive for NETs or CD68. NET+ macrophages were calculated by dividing \%CD68 by $\%$ NETs. NLRP3 ${ }^{+}$, Caspase $-1^{+}$, and iNOS $^{+}$cells were divided by total cells (DAPI nuclear statining) to get \%total cells.

Cholesterol crystal images were acquired using an Axioscan microscope (Carl Zeiss, Jena, Germany) using a 10x air objective. Cholesterol crystal images were captured using polarized light and represented as \% plaque area.

Laser Capture Microscopy and RNA-seq: CD68 ${ }^{+}$cells were selected from plaques by laser capture microdissection (LCM) as previously described (31), and all LCM procedures were performed under RNase-free conditions. Briefly, aortic root sections were stained with hematoxylin-eosin, and foam cells identified under a microscope and verified by positive CD68 staining. Following LCM, RNA was isolated using the PicoPure Kit (Molecular Devices, Inc., Sunnyvale, CA), treated with DNase and the quality and quantity determined using an Agilent 2100 Bioanalyzer (Agilent Technologies, Santa Clara, CA). RNA was amplified (Automated Nugen Ovation Trio Low Input RNA (500pg)), sequenced (HiSeq 4000 Paired-End, Illumina), and sequencing results analyzed using Ingenuity Pathway Analysis (IPA; Qiagen). Cutoffs 
of -0.5 to $0.5 \log 2$ fold change yielded 7337 analyses-ready molecules of which 4046 were down- and 3291 up-regulated in NET+ versus NET- areas. The data discussed in this report have been deposited in NCBI's Gene Expression Omnibus (32) and are accessible through GEO Series accession number GSE145200 (https://www.ncbi.nlm.nih.gov/geo/query/acc.cgi?acc=GSE145200).

To obtain transcripts of bone-marrow derived macrophages (BMDM), bone marrow cells were isolated by flushing cells from the femurs and tibiae of wild-type C57BL6 mice. Cells were differentiated into BMDMs in $4.5 \mathrm{~g} / \mathrm{L}$ glucose DMEM (Lonza) with $20 \%$ fetal bovine serum (FBS), 1\% penicillin/streptomycin, and murine M-CSF (10 ng/mL; \#315-02, PeproTech) at $37^{\circ} \mathrm{C}$ and $5 \% \mathrm{CO}_{2}$ for 7 days. Cells were lysed with Buffer RLT (Qiagen) containing $10 \%$ betamercaptoethanol and the Norgen Animal Tissue RNA Purification Kit (\#25700, Norgen Biotek) was used to isolate RNA from cells. The Dynabeads mRNA Direct Purification Kit (\#61011, ThermoFisher Scientific) was used to isolate mRNA transcripts using Poly-A selection, and cDNA synthesis was performed using SuperScript II Reverse Transcriptase (\#18064014, ThermoFisher Scientific). Libraries were prepared from $50 \mathrm{ng}$ of each cDNA sample using the Nextera DNA library prep kit (\#FC-121-1031, Illumina). Samples were sequenced on the Illumina HiSeq 2000 using 50 paired-end reads.

In the case of vascular smooth muscle cells (VSMC), cells were prepared from wildtype C57BL6 mice as in (33), and the RNA isolated and sequenced as described above.

Statistics: Data are expressed as mean \pm SEM. Data were tested for normality and equal variance and analyzed by the appropriate parametric or non-parametric test in GraphPad Prism 7 as stated in each figure. $P \leq 0.05$ was considered significant.

\section{Results}

NET formation is associated with a proinflammatory macrophage phenotype

Although NETs have been associated with macrophage activation and potentiating plaque formation (10), their molecular effects on plaque macrophage phenotype in vivo have not been directly determined. To address this gap, we collected macrophages 
(CD68+ cells) in NET positive (NET+) and NET negative (NET-) atherosclerotic plaque areas (Figure 1A, Supplemental Figure 1A) by laser capture microdissection (LCM) in Ldlr $r^{-}$mice after 16 weeks of western diet feeding. Collected macrophages provided material for profiling by RNA sequencing to characterize the phenotypical features of macrophages in NET+ areas in atherosclerotic plaques (Figure 1B, C).

Consistent with a proinflammatory phenotype, glycolysis and inflammasome pathways were enriched in macrophages isolated from NET+ areas relative to those in NETregions (Figure 1D, E). Confirmation of inflammasome activation was achieved by immunohistochemical staining for NLRP3 and Caspase-1, and quantification of their localization in NET+ versus NET- macrophage regions (Figure 1F). Inflammasome activation was found to be significantly associated with macrophages present in NET+ areas $(P=0.03$, Figure $1 \mathrm{G})$. In addition to the enrichment in inflammasome-related genes in the NET+ areas, the enrichment of glycolytic markers also indicates a switch to a proinflammatory M1-like macrophage phenotype (34). Indeed, based on standard markers, the CD68+ cells from NET+ vs. NET- areas were enriched in M1 (3.6-fold increased, inos), and suppression of M2 (2-fold decrease (Cd206)) characteristics. Further, immunofluorescence staining of iNOS confirmed increased proinflammatory macrophages in NET+ plaque areas $(P=0.055$, Figure $1 \mathrm{H})$. 
A.

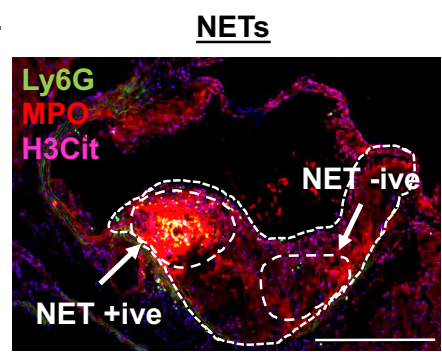

Macrophages

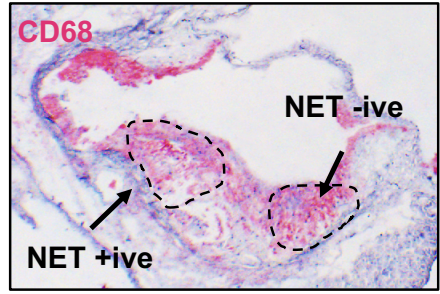

B.

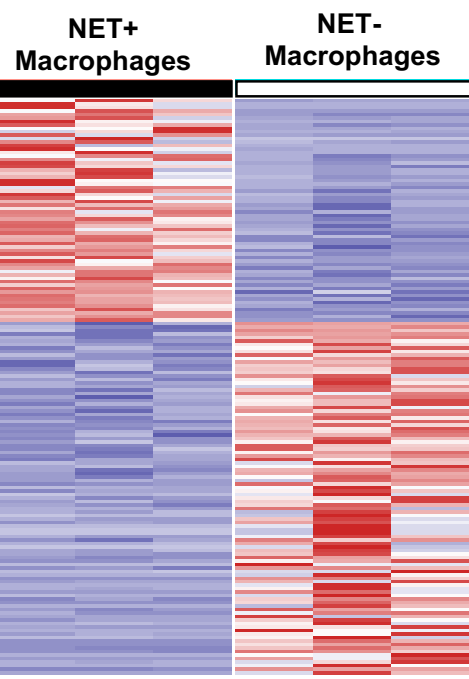

C.

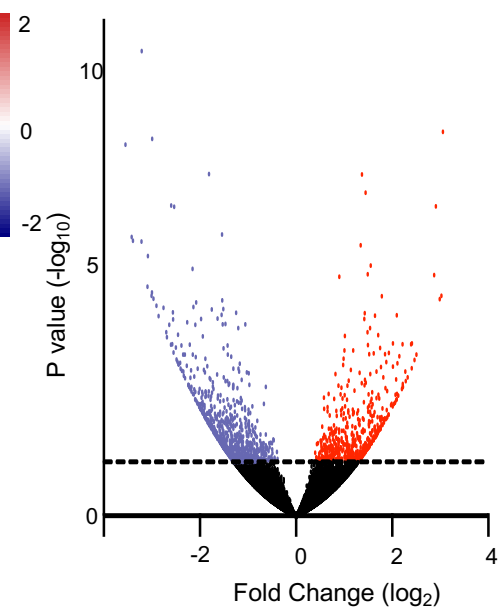

D. Glycolysis

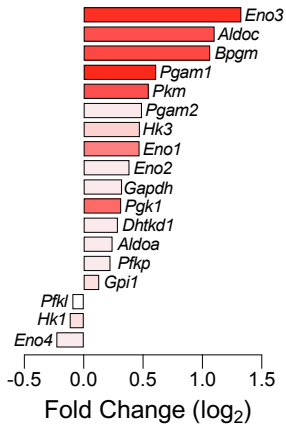

E. Inflammasome

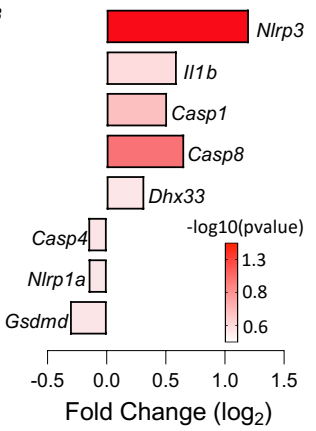

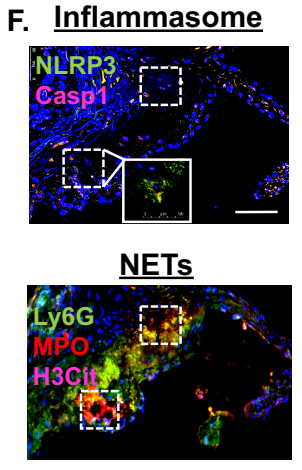

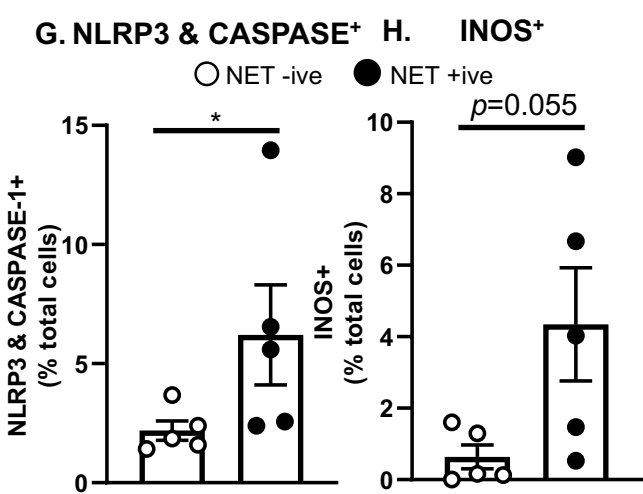

Figure 1. Neutrophil extracellular traps (NETs) in atherosclerotic plaques skew macrophages to a proinflammatory phenotype.

(A) Representative atherosclerotic plaque section stained for neutrophil extracellular traps (NETs) as determined by colocalization of myeloperoxidase (MPO), the lymphocyte antigen 6 complex locus G6D (Ly6G), citrullinated histone H3 (H3Cit) and macrophages (CD68). CD68+ cells were collected via laser capture microdissection (LCM) in NET positive (NET+) and NET negative (NET-

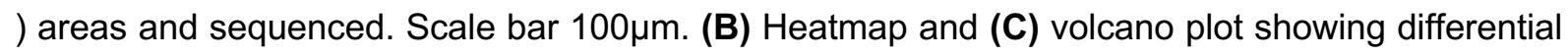
gene expression in NET+ versus NET- macrophages. (D) Upregulation of the glycolysis and (E) inflammasome pathways in NET+ compared to NET- macrophages determined using Ingenuity Pathway Analysis. (F) Immunofluorescence staining and (G) quantification confirming the inflammasome pathway activation (NLR family pyrin domain containing protein $3\left(\mathrm{NLRP}^{+}\right)$, caspase $1^{+}$) in NETs+ area (bottom), scale bar $50 \mu \mathrm{m}$. (H) Quantification of iNOS immunofluorescence staining showing an increase in iNOS in NET+ versus NET- Area (\%DAPI). $\mathrm{N}=3$-5/group, ${ }^{*} \mathrm{P}<0.05$ using unpaired t-test. 
To exclude the possibility that the RNA from CD68+ cells selected from NET+ areas was contaminated by neutrophil RNA, we compared the reads for CD68 to that of the neutrophil markers $\mathrm{Ly} 6 \mathrm{~g}, \mathrm{Mpo}$ and neutrophil elastase $(\mathrm{Ne})$. Counts for Cd68 were 7500 -fold greater than that of neutrophil transcripts, confirming the specificity of our approach (Supplemental Figure 1B). Additionally, to exclude the possibility of significant contamination with smooth muscle cells (SMC), which can take on characteristics of macrophages when cholesterol-loaded in vitro (35) or in atherosclerotic plaques (36-38), we compared the transcript expression of Cd68 to the myeloid-specific transcript Ptpc (Cd45) and the smooth muscle cell markers myosin heavy chain-11 (Myh11) and calponin 1 (Cnn1) using the expression values from RNA seq data obtained in the present study, and in primary murine bone marrow-derived macrophage (BMDMs) and murine vascular SMCs. As shown in Supplemental Figure $1 \mathrm{C}$, the $C d 45 / C d 68$ ratio was comparable between the laser captured CD68+ cells and BMDMs, whereas Cd45 was undetectable in murine vascular SMCs. Further, compared to laser captured CD68+ cells, the Myh11/Cd68 and Cnn1/CD68 ratios are approximately 12 -fold higher in vascular SMCs. Overall, these data show that the data in Figure 1 are not confounded to a significant extent by contamination by other cell types and provide further support that NETs skew macrophages towards a proinflammatory phenotype in atherosclerotic plaques.

Clearing NETs can overcome impaired atherosclerosis resolution in diabetic mice Neutrophils isolated from diabetic mice and humans are more prone to form NETs, with NET formation leading to impaired wound healing (24). We have also shown that even with lipid-lowering, maximal atherosclerosis resolution requires reduced macrophage inflammation (29). Thus, we hypothesized that the expected increase in plaque NETs in diabetic mice would impair atherosclerosis resolution due to their proinflammatory effects on macrophages (Figure 1), and that resolution in diabetic mice would improve by reducing NETs.

To test this, $L d I r^{\prime-}$ mice were fed a Western diet for 16 weeks to generate advanced atherosclerotic lesions, and atherosclerosis resolution was induced, as in a previous study (5), by feeding the mice a chow diet for four weeks, which resulted in decreased plasma lipid levels (Figure 2A; Table 1). A subset of mice was made diabetic by 
injection of streptozotocin. Since NETs are predominantly degraded by exonucleases (39), mice received DNase1 or vehicle injections every other day during the resolution period. In total, we compared baseline (mice sacrificed after 16 weeks on a western diet) and four regression groups: control, control + DNase1, STZ-induced diabetes, and diabetes + DNase1.

Table 1. Total plasma cholesterol and blood glucose levels

\begin{tabular}{l|c|c} 
& $\begin{array}{c}\text { Cholesterol } \\
(\mathrm{mg} / \mathrm{dL})\end{array}$ & $\begin{array}{c}\text { Blood Glucose } \\
(\mathrm{mg} / \mathrm{dL})\end{array}$ \\
\hline Baseline & $1000 \pm 327.7$ & $108.6 \pm 32.3^{\circ}$ \\
\cline { 2 - 3 } Regression & $221.6 \pm 71.1^{*}$ & $123.5 \pm 31.3^{\circ}$ \\
\cline { 2 - 3 } Regression + DNase1 & $216.4 \pm 28.5^{*}$ & $175.0 \pm 26.7^{\circ}$ \\
\cline { 2 - 3 } Diabetes & $325.1 \pm 83.4^{*}$ & $450.5 \pm 79.5$ \\
\cline { 2 - 3 } Diabetes + DNase1 & $323.3 \pm 96.4^{*}$ & $453.8 \pm 99.1$
\end{tabular}

Data shown as mean $\pm S D, n=5$-13/group, 1-way ANOVA, ${ }^{*}<<0.0001$ compared to Baseline; ${ }^{\circ} \mathrm{P}<0.0001$ compared to Diabetes

Neither STZ nor DNase1 affected total plasma cholesterol levels in the four resolution groups, with the levels in each significantly reduced when compared to baseline mice (Table 1). Plasma glucose levels were increased in the STZ groups only (Table 1). To evaluate NET content, we performed standard NET staining (MPO, $\mathrm{H} 3 \mathrm{Cit}$ and the neutrophil marker Ly6G; Figure 2B) and quantified NETs as \% plaque area (Figure 2C). Strikingly, in the non-diabetic mice, we observed a decrease in total plaque NETs area in the control and DNase 1 resolution groups versus baseline $(5.7 \%$ vs. $\sim 1.5 \%$ of plaque area), showing that NETs can resolve spontaneously in a non-hyperlipidemic environment. As expected, NETs did not resolve spontaneously in hyperglycemic conditions, but, notably, we observed a substantial decrease in NET content in the diabetes + DNase 1 group ( $7.0 \%$ vs. $2.3 \%$ of plaque area, or a $68 \%$ reduction). 
A.

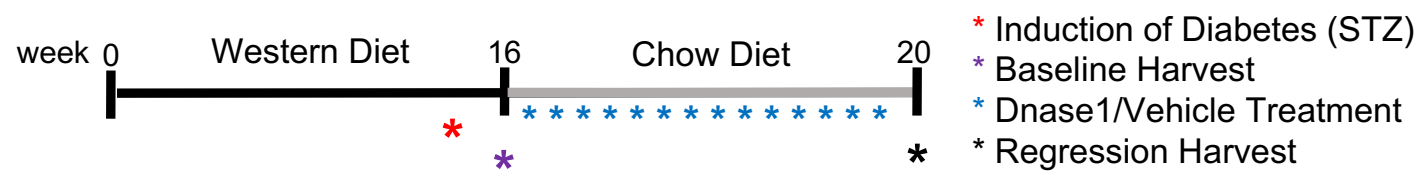

B.

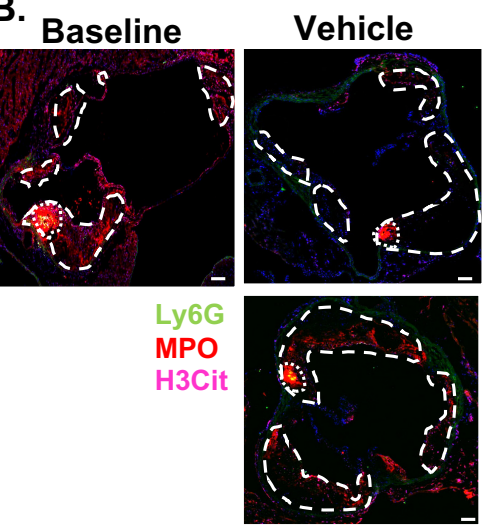

DNase1

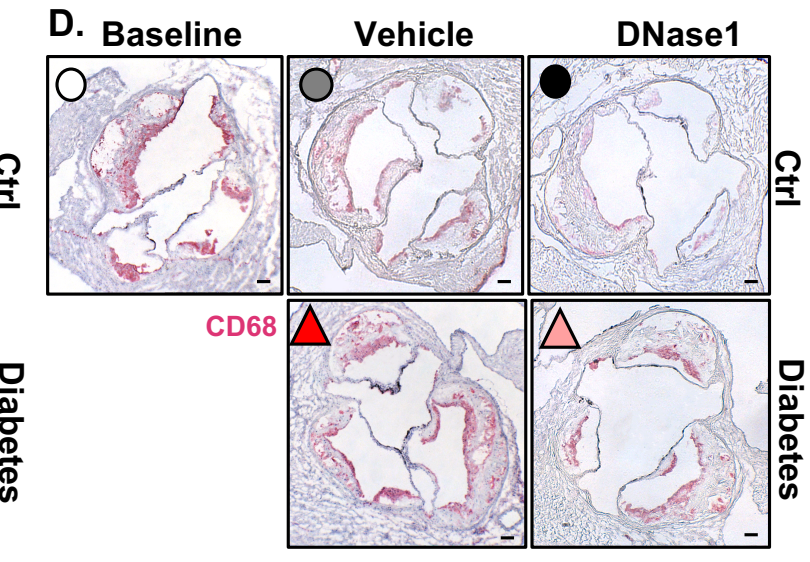

C.

NETs
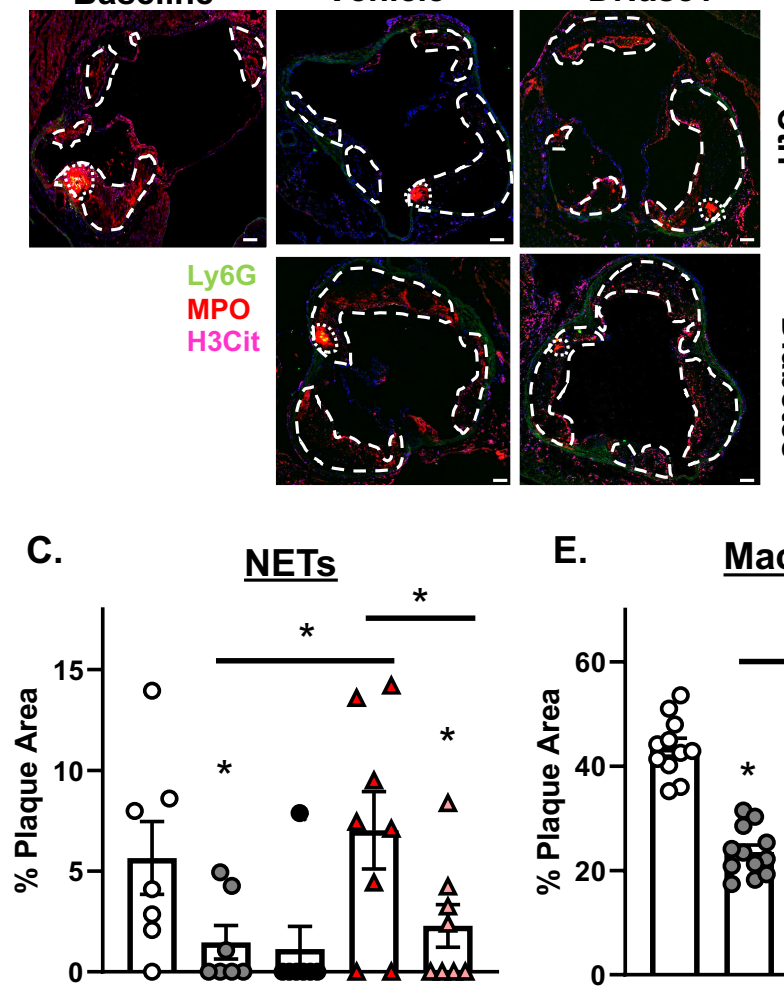

E.

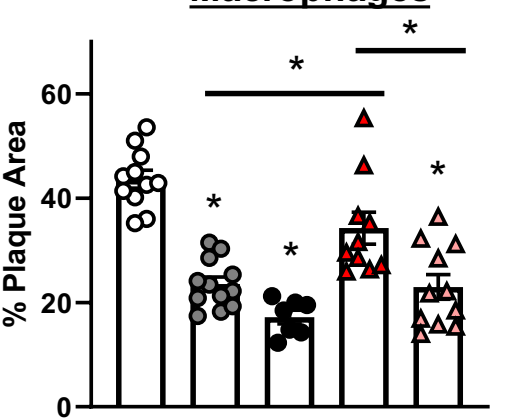

OBaseline OReg. Reg. + DNase1 $\triangle$ Diabetes $\triangle$ Diabetes + DNase1

Figure 2. DNase1 treatment promotes atherosclerosis regression in diabetic mice.

(A) Male $L d l r^{\prime}$ - mice were fed a western diet for 16 weeks to develop baseline plaques. At week 15 a subset of mice received streptozocin injections to induce diabetes. At week 16 a subset of mice was harvested for baseline measures, and all other mice were switched to a chow diet to induce resolution of plaques. These mice were then split into 2 groups, and over a 4-week period received either DNase1 (62.5ug/mouse) or vehicle injections every other day. At week 20, mouse tissues were harvested for plaque analyses. (B) NETs staining and (C) quantification in aortic roots, as determined by composite staining of myeloperoxidase (MPO), the lymphocyte antigen 6 complex locus G6D (Ly6G) and citrullinated histone H3 (H3Cit); scale bar $100 \mu \mathrm{m}$. (D) Representative pictures and (E) quantification of CD68 staining as a marker of plaque macrophage content. Scale bar $100 \mu \mathrm{m}$. Data are shown as mean $\pm \mathrm{SEM}, \mathrm{N}=7$-12/group * $\mathrm{P}<0.05$, 1-way ANOVA with Tukey's multiple comparison test. 
To determine if NETs content correlated negatively with atherosclerosis resolution, CD68+ macrophages were quantified and represented as the percentage of plaque area (\%CD68; Figure 2D-E). As expected, we found a significant decrease in lesion \%CD68 in control mice compared to the baseline group (43.6 \% vs. $24.1 \%)$. Atherosclerosis resolution was unchanged in control mice receiving DNase1, consistent with the spontaneous loss of NETs in those mice. In diabetic mice, the reduction of \%CD68 immunostaining was impaired (43.6 \% vs. $35.2 \%$ ), as expected (3). Notably, DNase1 treatment in diabetic mice showed significantly enhanced atherosclerosis resolution (35.2\% vs. $23.0 \%$ ), which was comparable to that of control mice.

To determine the relationships among the relative contributions to plaque characteristics by macrophages (above), extracellular matrix (as assessed by collagen) and necrotic core, additional plaque analyses were performed. As shown in Figure $3 \mathrm{~A}$, there was a significant reduction in plaque size in all of the resolution groups (Figure $3 \mathrm{~A}$ ) compared to baseline. Collagen content tended to increase relative to baseline, with statistical significance achieved in the resolution control group (Figure 3B). The necrotic core plaque areas were found to be similar in the baseline and the diabetes resolution groups, and significantly lower in the other resolution groups, including DNase1 treated diabetic mice (Figure 3C).

OBaseline OReg. Reg. + DNase1 $\triangle$ Diabetes $\triangle$ Diabetes + DNase1
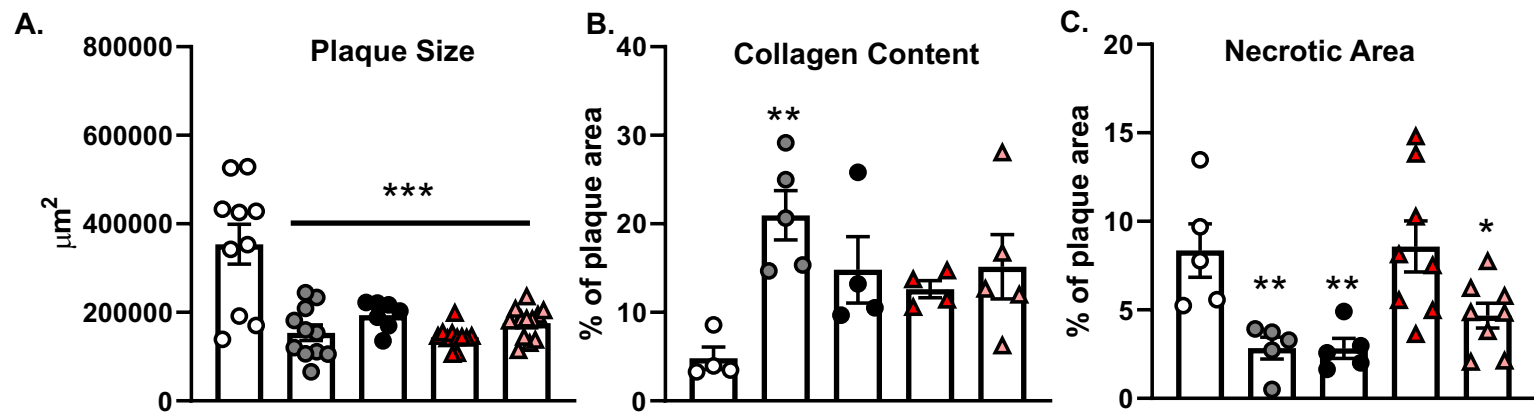

Figure 3. Effects of DNase1 treatment on plaque size, collagen content, and necrotic area in atherosclerotic plaques.

More analyses of mice described in Figure 2: Quantification of (A) plaque size, (B) Sirius red staining to determine collagen content and (C) plaque necrotic area. Data are shown as mean \pm SEM, N=4-12/group *P<0.05, ${ }^{* *} \mathrm{P}<0.005,{ }^{* * *} \mathrm{P}<0.001$ 1-way ANOVA with Dunnett's multiple comparison test compared to the baseline $(A, B)$ or diabetes $(C)$ group. 
DNase1 treatment reduces NET-induced plaque macrophage inflammation and promotes atherosclerosis resolution in diabetic mice

The results in Figure 2 showed DNase 1 treatment was able to overcome the impairment of atherosclerosis resolution by diabetes. Because a prominent feature of macrophages in NET+ areas is the induction of the inflammasome and other metabolic and transcriptomic changes characteristic of inflammation (Figure 1), we hypothesized that the benefits of NET clearance in diabetic mice (Figure 2) were associated with a reduction in the inflammatory phenotype of plaque macrophages. Consistent with this, the percentage of macrophages associated with plaque NETs was significantly decreased by $90 \%$, from 2.7 to $0.3 \%$ in the DNAse 1 treated diabetic mice compared to diabetic mice with no treatment (Figure 4A-B). To support this hypothesis more directly, we performed immunofluorescence staining for the inflmmasome markers NLRP3 and Caspase-1 (Figure 4C). Indeed, NLRP3 and Caspase-1 positive cells were significantly increased in diabetic mice, compared to the control group, and decreased with DNase1 treatment. Additionally, the increase in plaques of diabetic mice of the inflammatory macrophage marker iNOS was reversed with DNase1 treatment (Figure 4D). As we previously reported $(6,7)$, there was a significant increase in circulating neutrophils in diabetes. There was also a trend towards increased plaque neutrophils (Supplemental Figure 2A, B), with a suggestion that DNase1 reduced both increases. 

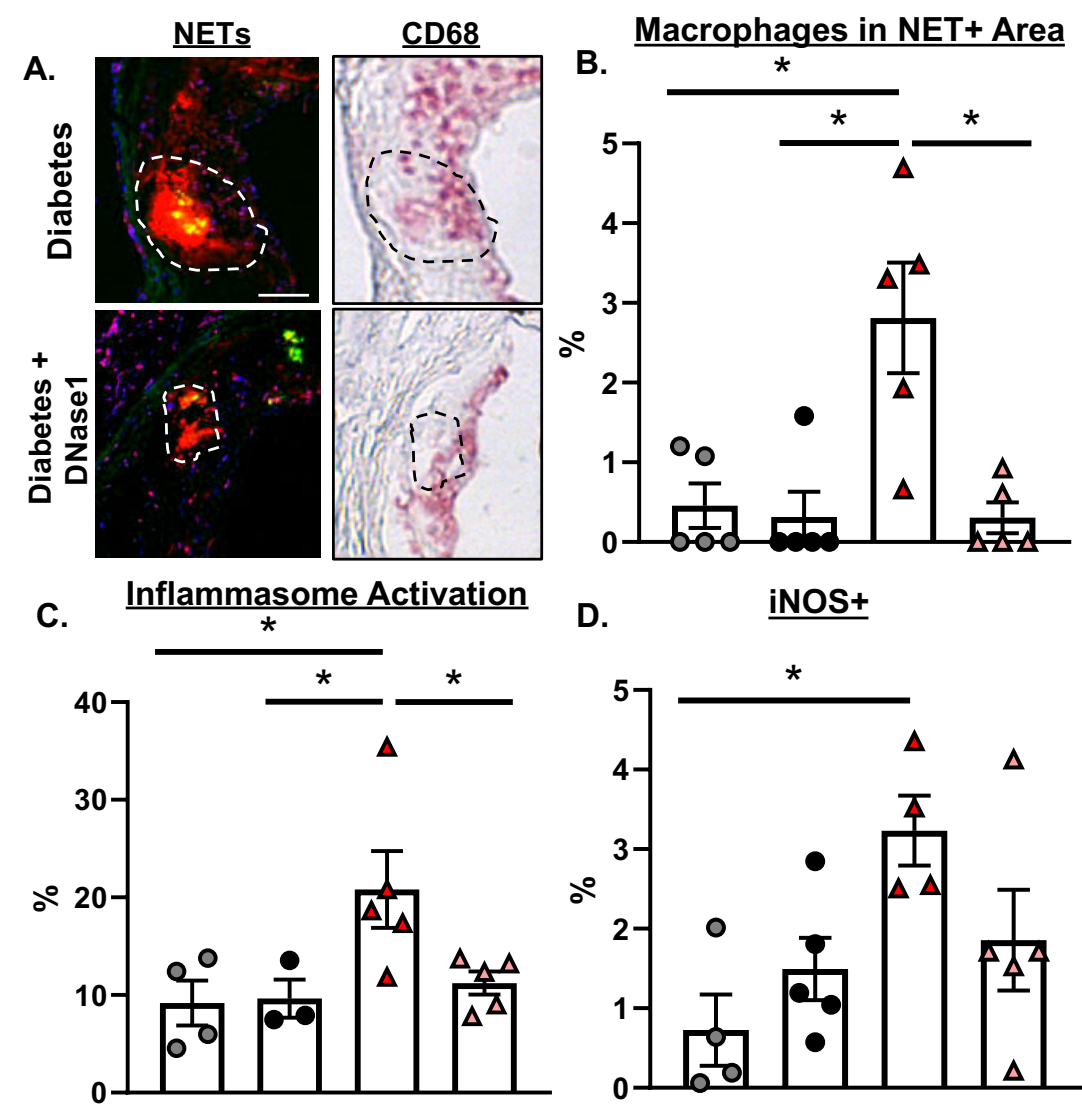

D. iNOS+

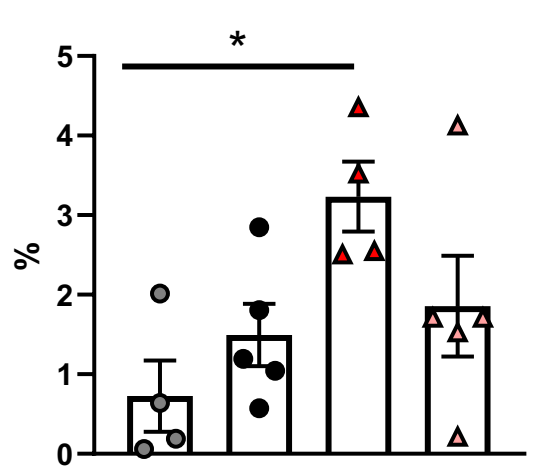

OReg. Reg. + DNase1 $\triangle$ Diabetes $\triangle$ Diabetes + DNase1

Figure 4. DNase1 treatment promotes atherosclerosis resolution in diabetic mice by reducing NETs-induced plaque macrophage inflammation.

More analyses of mice described in Figure 2: (A) Overlapping NET (left panel) and CD68 staining (right panel) in diabetic mice versus diabetic mice treated with DNase1, scale bar $50 \mu \mathrm{m}$. (B) Amount of macrophages (\%) co-localized with NETs. (C) Quantification of NLRP3 ${ }^{+}$, caspase- $1^{+}$ cells, and, (D) iNos ${ }^{+}$cells, all as \% DAPI staining of plaques. Data are shown as mean \pm SEM, N=35/group, * $\mathrm{P}<0.05,1$-Way ANOVA with Tukey's multiple comparison test.

Cholesterol crystals have been shown to be an inflammasome activator in atherosclerosis (40). Thus, we examined whether they were associated with the macrophage inflammation we observed and if DNase1 treatment affected their plaque content. Cholesterol crystals in atherosclerotic plaques were detected using polarized light (Figure $5 \mathrm{~A}$ ). As shown in Figure $5 \mathrm{~B}$, in the resolution groups, the cholesterol crystal content was $\sim 70 \%$ greater $(P=0.01)$ in the diabetes $(2.2 \%)$ vs. control mice $(1.3 \%)$. Interestingly, cholesterol crystals tended to be decreased in the diabetes + DNase1 compared to diabetes group $(P=0.06)$. 
A. Vehicle
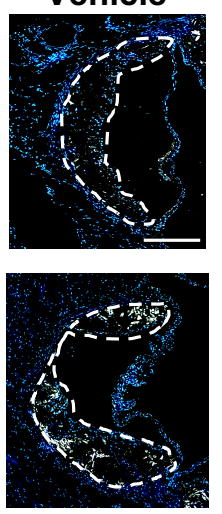

OReg.
DNase1
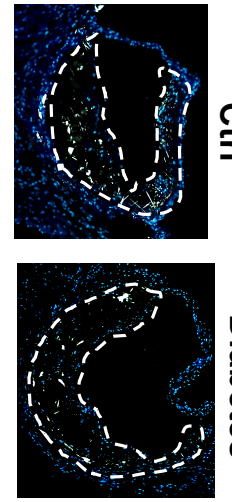

Reg. + DNase1
B. Cholesterol crystals

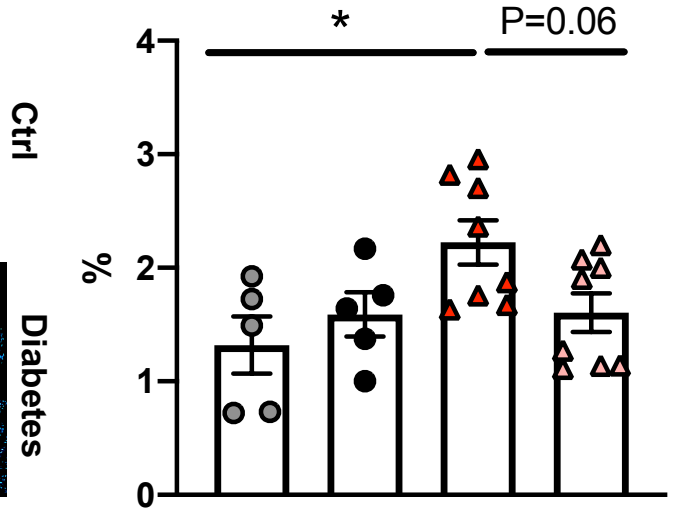

$\triangle$ Diabetes $\triangle$ Diabetes + DNase1

Figure 5. Effects of diabetes and DNAse1 treatment on cholesterol crystal content in resolving plaques.

More analyses of mice described in Figure 2: (A) Cholesterol crystal visualization (white), and (B) quantification (as \% plaque area) in atherosclerotic plaques with DAPI (blue) as counterstain; scale bar $100 \mu \mathrm{m}$. Data are shown as mean $\pm \mathrm{SEM}, \mathrm{N}=5$-8/group, ${ }^{*} \mathrm{P}<0.05$, 1 -Way ANOVA with Tukey's multiple comparison test.

\section{Discussion}

NETs are found in human and mouse plaques, and in mice are associated with increased atherosclerosis progression (10-12). They have also been shown to impair wound healing in diabetic mice (24). Though considered to be generally inflammatory, how NETs contribute to macrophage activation is incompletely understood (17). The present study addresses this gap in knowledge. By isolation of macrophages from NET positive and negative plaque areas, we identify that NETs induce a proinflammatory "M1-like" macrophage phenotype, as evidenced at the molecular level by enriched transcripts characteristic of glycolysis and inflammasome activation and at the protein level by increases in relevant immunohistochemical markers. We also detected enhanced NET presence in diabetic plaques, consistent with a study in nonatherosclerotic mice that diabetes primes neutrophils for NET production (24).

The NET-macrophage interaction is a likely contributing factor to persistent macrophage inflammation in the plaques of diabetic mice even after lipid-lowering. This is supported by our current study, which finds a reduction in plaque inflammation and proinflammatory macrophages in lesions of diabetic mice following the degradation of 
NETs with DNase1 in conjunction with lipid-lowering. Further, we provide evidence that the resolution of plaque NETs in diabetic mice promotes a more stable appearance, as noted by a decrease in plaque necrotic area following DNase1 treatment. These data are consistent with the association between NET formation and human plaque instability $(12,41)$.

DNase1 reduces NETs by degradation of the chromatin fibers that comprise their backbone (14), which usually, but not always, reduces inflammation and disease severity in several models (42). Based on the results in the present study, we can add the resolution of atherosclerosis in diabetes to the list of conditions that are improved by reducing NET content. In addition to the direct effects in plaques by the reduction of NETs (e.g., reduced inflammasome activation in plaque macrophages), there are probably indirect benefits as well. For example, inflammation in plaques also increases neutrophil recruitment, further amplifying inflammation (43). This reasoning likely provides the logical link between the reduction in plaque inflammation in diabetic mice (Figure 4) with the data suggesting that the increased circulating and plaque neutrophil levels in the diabetic mice were partially suppressed by DNase1 treatment (Supplemental Figure 2).

The present results showing a powerful effect of NETs on macrophage inflammasome activation led us to consider this finding in the context of another mediator of inflammasome activation in atherosclerosis, namely cholesterol crystals (40). The current understanding is that the inflammasome pathway requires two signals for full activation. Warnatsch et al. (10) have shown that NETs are a priming cue, with the second signal being cholesterol crystals (9). Furthermore, crystal formation and inflammasome activation were recently shown by Westerterp et al. to be promoted by reduced cholesterol efflux via ATP-binding cassette transporter A1/G1 (ABCA1/ABCG1) (44). Additionally, cholesterol crystals are not only the second signal for inflammasome activation, but they also stimulate NETosis (10).

As shown in Figure 5, cholesterol crystals were increased in diabetic mice relative to the other resolution groups, which is consistent with hyperglycemia reducing cholesterol efflux in macrophages and their precursors by decreasing ABCA1/ABCG1 
expression (e.g., $(6,7)$ ). Taken together, our and the published data imply that cholesterol crystals could play a dual role in atherosclerosis, namely to promote NETosis, which provides multiple priming cues (first signals) for macrophage inflammasome activation, and also to serve as the second signal to complete the activation process in the primed macrophages. DNase1 treatment, by clearance of NETs, would reduce both types of macrophage inflammasome signals. This results in less macrophage inflammation, which we have shown is required to promote maximal atherosclerosis resolution after lipid-lowering (29).

In summary, we have shown for the first time the molecular impact of NETs on plaque macrophages, especially on their inflammasome and glycolytic pathways. We also show that NETs formed during atherosclerosis progression in non-diabetic mice can spontaneously resolve upon the correction of hyperlipidemia. In contrast, they persist in the setting of diabetes and impair atherosclerosis resolution, likely due to their activation of macrophages and exacerbation of the general inflammatory environment in the plaque. Notably, DNase1 was an effective treatment to improve atherosclerosis resolution in the face of ongoing hyperglycemia. Because the rate of CV disease remains higher in people with versus without diabetes after similar lipid-lowering, the results suggest not only a basis for this but also the therapeutic potential of NET reduction, especially in those whose diabetes is not well controlled.

\section{Author Contributions}

T.J., T.J.B. and E.A.F. designed experiments. T.J. and T.J.B performed the experiments and analyses. E.B. assisted with the RNA seq analyses. A.Q. helped with the analysis performed in Figure 2. X.W. and J.A. performed cholesterol crystal visualization and quantification. M.V. added BMDM sequencing data. T.J., T.J.B., J.A., E.A.F wrote and edited the manuscript.

\section{Acknowledgments}

We appreciate the technical contributions of Yoscar Ogando and Felix Zhou. The RNA sequencing was done by the Genome Technology Center, a shared resource that is partially supported by the Cancer Center Support Grant P30CA016087 at the Laura and Isaac Permutter Cancer Center. 
Funding was provided by American Heart Association (18PRE33990436, TJ; 18CDA34110203AHA, TJB, 16SDG27550012, JA), the American Society of Hematology (18-A0-00-1001884, TJB), and the NIH (HL147252, JA; and for EAF, TJ, TJB, R01 DK095684, R01 HL117226, R01 HL084312, R01 HL129433, P01 HL092969, and P01 HL131481).

\section{Conflicts of Interest}

Based on a review of the criteria of $\mathrm{JCl}$ Insight, the only author with a disclosure is Edward A. Fisher, who reports receiving income $>\$ 10,000$ from being an expert witness on behalf of Amgen (PCSK9 antibodies) and Dr. Reddy's Laboratories (eicosapentaenoic acid). 


\section{References}

1. Grau AJ, Boddy AW, Dukovic DA, Buggle F, Lichy C, Brandt T, et al. Leukocyte count as an independent predictor of recurrent ischemic events. Stroke. 2004;35(5):1147-52.

2. Sweetnam PM, Thomas HF, Yarnell JW, Baker IA, and Elwood PC. Total and differential leukocyte counts as predictors of ischemic heart disease: the Caerphilly and Speedwell studies. Am J Epidemiol. 1997;145(5):416-21.

3. Parathath S, Grauer L, Huang L-S, Sanson M, Goldberg I, and Fisher E. Diabetes mellitus adversely affects macrophages during atherosclerotic plaque regression in mice. Diabetes. 2011;60:1759-69.

4. Gaudreault N, Kumar N, Olivas VR, Eberle D, Stephens K, and Raffai RL. Hyperglycemia impairs atherosclerosis regression in mice. Am J Pathol. 2013;183(6):1981-92.

5. Nagareddy PR, Murphy AJ, Stirzaker RA, Hu Y, Yu S, Miller RG, et al. Hyperglycemia promotes myelopoiesis and impairs the resolution of atherosclerosis. Cell Metab. 2013;17(5):695-708.

6. Barrett TJ, Distel E, Murphy AJ, Hu J, Garshick MS, Ogando Y, et al. Apolipoprotein AI) Promotes Atherosclerosis Regression in Diabetic Mice by Suppressing Myelopoiesis and Plaque Inflammation. Circulation. 2019;140(14):1170-84.

7. Distel E, Barrett TJ, Chung K, Girgis NM, Parathath S, Essau CC, et al. miR33 inhibition overcomes deleterious effects of diabetes mellitus on atherosclerosis plaque regression in mice. Circ Res. 2014;115(9):759-69.

8. Doring Y, Drechsler M, Soehnlein 0, and Weber C. Neutrophils in atherosclerosis: from mice to man. Arterioscler Thromb Vasc Biol. 2015;35(2):288-95.

9. Van Avondt K, Maegdefessel L, and Soehnlein O. Therapeutic Targeting of Neutrophil Extracellular Traps in Atherogenic Inflammation. Thromb Haemost. 2019;119(4):542-52.

10. Warnatsch A, Ioannou M, Wang Q, and Papayannopoulos V. Inflammation. Neutrophil extracellular traps license macrophages for cytokine production in atherosclerosis. Science. 2015;349(6245):316-20.

11. Megens RT, Vijayan S, Lievens D, Doring Y, van Zandvoort MA, Grommes J, et al. Presence of luminal neutrophil extracellular traps in atherosclerosis. Thromb Haemost. 2012;107(3):597-8.

12. Quillard T, Araujo HA, Franck G, Shvartz E, Sukhova G, and Libby P. TLR2 and neutrophils potentiate endothelial stress, apoptosis and detachment: implications for superficial erosion. Eur Heart J. 2015;36(22):1394-404.

13. Castanheira FVS, and Kubes P. Neutrophils and NETs in modulating acute and chronic inflammation. Blood. 2019;133(20):2178-85.

14. Brinkmann V, Reichard U, Goosmann C, Fauler B, Uhlemann Y, Weiss DS, et al. Neutrophil extracellular traps kill bacteria. Science. 2004;303(5663):1532-5.

15. Qi H, Yang S, and Zhang L. Neutrophil Extracellular Traps and Endothelial Dysfunction in Atherosclerosis and Thrombosis. Front Immunol. 2017;8:928.

16. Soehnlein O, Zernecke A, Eriksson EE, Rothfuchs AG, Pham CT, Herwald H, et al. Neutrophil secretion products pave the way for inflammatory monocytes. Blood. 2008;112(4):1461-71. 
17. Soehnlein O, Ortega-Gomez A, Doring Y, and Weber C. Neutrophil-macrophage interplay in atherosclerosis: protease-mediated cytokine processing versus NET release. Thromb Haemost. 2015;114(4):866-7.

18. Knight JS, Luo W, O'Dell AA, Yalavarthi S, Zhao W, Subramanian V, et al. Peptidylarginine deiminase inhibition reduces vascular damage and modulates innate immune responses in murine models of atherosclerosis. Circ Res. 2014;114(6):947-56.

19. Borissoff JI, Joosen IA, Versteylen MO, Brill A, Fuchs TA, Savchenko AS, et al. Elevated levels of circulating DNA and chromatin are independently associated with severe coronary atherosclerosis and a prothrombotic state. Arterioscler Thromb Vasc Biol. 2013;33(8):2032-40.

20. Rodondi N, Marques-Vidal P, Butler J, Sutton-Tyrrell K, Cornuz J, Satterfield S, et al. Markers of atherosclerosis and inflammation for prediction of coronary heart disease in older adults. Am J Epidemiol. 2010;171(5):540-9.

21. Armitage J, and Bowman L. Cardiovascular outcomes among participants with diabetes in the recent large statin trials. Curr Opin Lipidol. 2004;15(4):439-46.

22. Nicholls SJ, Tuzcu EM, Kalidindi S, Wolski K, Moon KW, Sipahi I, et al. Effect of diabetes on progression of coronary atherosclerosis and arterial remodeling: a pooled analysis of 5 intravascular ultrasound trials. J Am Coll Cardiol. 2008;52(4):255-62.

23. Menegazzo L, Ciciliot S, Poncina N, Mazzucato M, Persano M, Bonora B, et al. NETosis is induced by high glucose and associated with type 2 diabetes. Acta Diabetol. 2015;52(3):497-503.

24. Wong SL, Demers M, Martinod K, Gallant M, Wang Y, Goldfine AB, et al. Diabetes primes neutrophils to undergo NETosis, which impairs wound healing. Nat Med. 2015;21(7):815-9.

25. Rodriguez-Espinosa 0, Rojas-Espinosa O, Moreno-Altamirano MM, Lopez-Villegas EO, and Sanchez-Garcia FJ. Metabolic requirements for neutrophil extracellular traps formation. Immunology. 2015;145(2):213-24.

26. de Ferranti SD, de Boer IH, Fonseca V, Fox CS, Golden SH, Lavie CJ, et al. Type 1 diabetes mellitus and cardiovascular disease: a scientific statement from the American Heart Association and American Diabetes Association. Circulation. 2014;130(13):1110-30.

27. Ibrahim M, Tuomilehto J, Aschner P, Beseler L, Cahn A, Eckel RH, et al. Global status of diabetes prevention and prospects for action: A consensus statement. Diabetes Metab Res Rev. 2018;34(6):e3021.

28. Barrett TJ, Murphy AJ, Goldberg IJ, and Fisher EA. Diabetes-mediated myelopoiesis and the relationship to cardiovascular risk. Ann N Y Acad Sci. 2017;1402(1):31-42.

29. Rahman K, Vengrenyuk Y, Ramsey SA, Vila NR, Girgis NM, Liu J, et al. Inflammatory Ly6Chi monocytes and their conversion to M2 macrophages drive atherosclerosis regression. J Clin Invest. 2017;127(8):2904-15.

30. Yuan C, Hu J, Parathath S, Grauer L, Cassella CB, Bagdasarov S, et al. Human Aldose Reductase Expression Prevents Atherosclerosis Regression in Diabetic Mice. Diabetes. 2018;67(9):1880-91.

31. Trogan E, Choudhury RP, Dansky HM, Rong JX, Breslow JL, and Fisher EA. Laser capture microdissection analysis of gene expression in macrophages from 
atherosclerotic lesions of apolipoprotein E-deficient mice. Proc Natl Acad Sci U S A. 2002;99(4):2234-9.

32. Edgar R, Domrachev M, and Lash AE. Gene Expression Omnibus: NCBI gene expression and hybridization array data repository. Nucleic Acids Res. 2002;30(1):207-10.

33. Vengrenyuk Y, Nishi H, Long X, Ouimet M, Savji N, Martinez FO, et al. Cholesterol loading reprograms the microRNA-143/145-myocardin axis to convert aortic smooth muscle cells to a dysfunctional macrophage-like phenotype. Arterioscler Thromb Vasc Biol. 2015;35(3):535-46.

34. Buck MD, Sowell RT, Kaech SM, and Pearce EL. Metabolic Instruction of Immunity. Cell. 2017;169(4):570-86.

35. Rong JX, Shapiro M, Trogan E, and Fisher EA. Transdifferentiation of mouse aortic smooth muscle cells to a macrophage-like state after cholesterol loading. Proc Natl Acad Sci U S A. 2003;100(23):13531-6.

36. Feil S, Fehrenbacher B, Lukowski R, Essmann F, Schulze-Osthoff K, Schaller M, et al. Transdifferentiation of vascular smooth muscle cells to macrophage-like cells during atherogenesis. Circ Res. 2014;115(7):662-7.

37. Allahverdian S, Chehroudi AC, McManus BM, Abraham T, and Francis GA. Contribution of intimal smooth muscle cells to cholesterol accumulation and macrophage-like cells in human atherosclerosis. Circulation. 2014;129(15):15519.

38. Shankman LS, Gomez D, Cherepanova OA, Salmon M, Alencar GF, Haskins RM, et al. KLF4-dependent phenotypic modulation of smooth muscle cells has a key role in atherosclerotic plaque pathogenesis. Nat Med. 2015;21(6):628-37.

39. Fuchs TA, Abed U, Goosmann C, Hurwitz R, Schulze I, Wahn V, et al. Novel cell death program leads to neutrophil extracellular traps. The Journal of cell biology. 2007;176(2):231-41.

40. Duewell P, Kono H, Rayner KJ, Sirois CM, Vladimer G, Bauernfeind FG, et al. NLRP3 inflammasomes are required for atherogenesis and activated by cholesterol crystals. Nature. 2010;464(7293):1357-61.

41. Pertiwi KR, van der Wal AC, Pabittei DR, Mackaaij C, van Leeuwen MB, Li X, et al. Neutrophil Extracellular Traps Participate in All Different Types of Thrombotic and Haemorrhagic Complications of Coronary Atherosclerosis. Thromb Haemost. 2018;118(6):1078-87.

42. Jorch SK, and Kubes P. An emerging role for neutrophil extracellular traps in noninfectious disease. Nat Med. 2017;23(3):279-87.

43. Miller LS, O'Connell RM, Gutierrez MA, Pietras EM, Shahangian A, Gross CE, et al. MyD88 mediates neutrophil recruitment initiated by IL-1R but not TLR2 activation in immunity against Staphylococcus aureus. Immunity. 2006;24(1):7991.

44. Westerterp M, Fotakis P, Ouimet M, Bochem AE, Zhang H, Molusky MM, et al. Cholesterol Efflux Pathways Suppress Inflammasome Activation, NETosis, and Atherogenesis. Circulation. 2018;138(9):898-912. 


\section{Supplemental}

A.

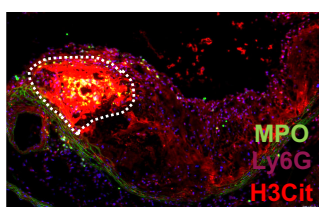

Merge (NETs)
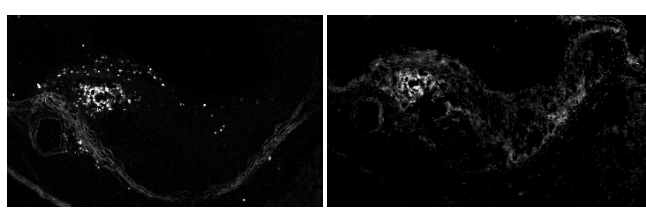

Ly6G

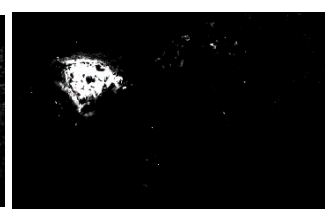

H3Cit

B.

c.
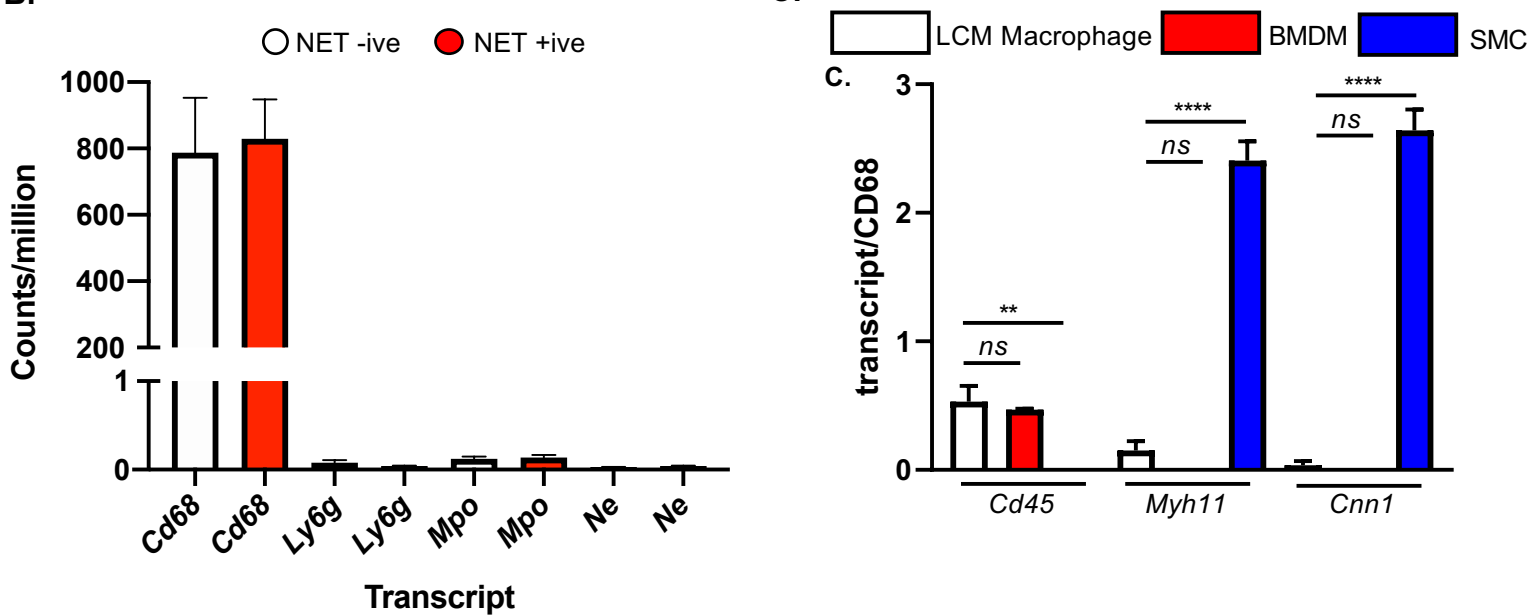

\section{Supplemental Figure 1}

(A) Atherosclerotic plaque section stained for neutrophil extracellular traps (NETs) as determined by colocalization of MPO (left), Ly6G (middle), H3Cit (right). (B) Macrophage (Cd68) and neutrophil ( $L y 6 g, M p o$, neutrophil elastase $(\mathrm{Ne})$ ) markers detected with RNA sequencing (counts/million). (C) Expression of macrophage (Cd45) and smooth muscle cell (Myosin Heavy Chain 11, Myh11; Calponin 1, Cnn1) transcripts in isolated LCM macrophages, murine bone marrow-derived macrophages (BMDM)s and murine vascular smooth muscle cells (SMC)s, as determined by RNAseq and represented as transcript/CD68 ratio. ${ }^{* *} \mathrm{P}<0.005,{ }^{* * *} \mathrm{P}<0.0001$ 1-way ANOVA with Dunnett's multiple comparison test, $n=3 /$ cell type. 
OReg. $\triangle$ Reg + DNase1 $\triangle$ Diabetes $\triangle$ Diabetes + DNase1

A.

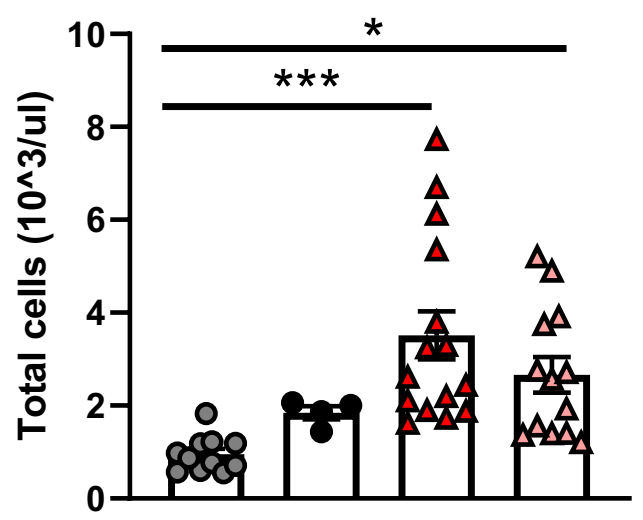

B.

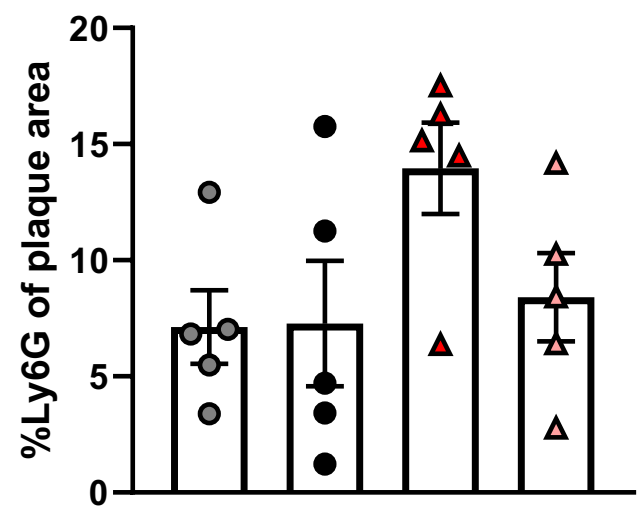

\section{Supplemental Figure 2}

(A) Circulating neutrophil counts and (B) plaque neutrophil contents. Data are shown as mean \pm SEM, $N=4-15 /$ group ${ }^{*} \mathrm{P}<0.05$, ${ }^{* * *} \mathrm{P}<0.0011$-way ANOVA with Dunnett's multiple comparison test. 


\section{6}

Loss of DNase1, DNase1L3 or both does not significantly change atherosclerotic neutrophil extracellular traps (NETs) content, or affect atherosclerosis

Tatjana Josefs, Tarik Zahr, Lee Serpas, Kristiaan Wouters, Boris Reizis, Edward A. Fisher

Pilot Study 


\begin{abstract}
Rationale: Neutrophil extracellular traps (NETs) are a pro-inflammatory component of atherosclerotic plaques. NETs can be cleared by endogenous endonucleases. Until now, it remains unclear whether the endonucleases deoxyribonuclease 1 (DNase1) and DNase1 Like 3 (DNase1L3) affect NET formation in atherosclerotic plaques and influence plaque progression and regression. We hypothesized that 1) single-deficiency of these DNases increases NET formation, with the most prominent effects in the double-deficient mice, and, 2) the increase in NETs enhances atherosclerosis progression and impairs regression.
\end{abstract}

Objective: To investigate the role of DNase1 and DNase1L3 in atherosclerosis progression and regression

Approach \& Results: After the progression period of 20 weeks on western diet, plasma cholesterol levels were relatively low $(\sim 600 \mathrm{mg} / \mathrm{dL})$, especially in the $D N a s e 1 L 3^{-/}$mice $(\sim 470 \mathrm{mg} / \mathrm{dL})$. Compared to control (1.5 $\pm 1.0 \%$ plaque area) animals, NET formation tended to be increased in the double-deficient mice $(3.3 \pm 1.4 \%$ plaque area) and was unaffected in DNase 1/- mice (0.7 $\pm 0.3 \%$ plaque area). NET formation was absent in DNase $1 \mathrm{~L}^{-/-}$mice $(0.0 \pm 0.0 \%$ plaque area). After the regression period of 4 weeks on chow diet, plasma cholesterol dropped to $\sim 100 \mathrm{mg} / \mathrm{dL}$ without affecting NET area and plaque size. Macrophage content (\%plaque area) significantly decreased during regression, but we did not find differences between single- or double-deficient mice compared to control.

Conclusion: Loss of DNase1, DNase1L3 or both does not significantly change NET content, worsen atherosclerosis progression or impairs regression. Notably, NET formation was limited likely due to rather low cholesterol level leading to insignificant results. Future studies need to be performed using a more severe atherosclerosis model and higher $\mathrm{n}$. 


\section{Introduction}

Two major endonucleases are present in the circulation and are members of the deoxyribonuclease 1 (DNase1) protein family, i.e. DNase1 and DNase1 Like 3 (DNase1L3). DNase1 is mainly expressed by exocrine cells of salivary glands, kidney and gut. ${ }^{1}$ DNase1L3 is expressed in immune cells, specifically in dendritic cells and macrophages, but also in Kupffer and gastrointestinal cells. ${ }^{2,3}$ DNases are critical for DNA degradation and are required for healthy development and function. The role of these endonucleases in autoimmune and inflammatory diseases has been intensively studied, given their ability to degrade neutrophil extracellular traps (NETs).

NETs are web-like structures expelled by neutrophils upon activation by pathogens or sterile inflammatory signals (e.g. autoantibodies, oxLDL). ${ }^{4-7}$ These NETs consist of cytosolic and nuclear material including histones, neutrophil elastase (NE), myeloperoxidase (MPO), proteinase 3 (PR3), cathepsin and gelatinase and are part of the hosts' immune defense. ${ }^{4}$ However, NETs are implicated in several noninfectious diseases, such as Lupus or atherosclerosis and therefore, clearance by endonucleases might prevent inadvertent effects of prolonged NETs exposure. In vitro experiments showed that DNase1 or DNase1L3 can degrade NETs. ${ }^{8,9}$ Given the NETs composition, DNase1 seems to be more likely the main endonuclease for NET degradation as DNase1 only works in combination with proteases (e.g. neutrophil elastase), while DNase1L3 is inhibited by proteases. Additionally, DNase1 preferentially cleaves free DNA, while DNase1L3 targets DNA-protein complexes, e.g. nucleosomes. In line, DNase1 has been reported to be important for NET degradation in lupus erythematous (SLE) patients and is linked to its pathogenesis. ${ }^{9}$ Further, mutations in DNase1, leading to decreased DNase1 activity in the circulation, are associated with SLE severity. ${ }^{10,11}$

Next to autoimmune diseases, increasing evidence showed that NETs also play a pro-inflammatory role in atherosclerosis. ${ }^{12,13}$ Atherosclerosis is a lipid-driven chronic inflammatory disease and the underlying pathology of cardiovascular diseases (CVDs) causing the most deaths worldwide. Administering DNase1 in atherosclerotic apoE-/mice fed a high-fat ${ }^{14}$ or a high cholesterol ${ }^{15}$ diet was reported to lower atherosclerosis plaque size. In contrast, another study showed no benefit of DNase1 administration on atherosclerosis progression in apoE $E^{-/}$mice fed a high-fat diet for 4 months. ${ }^{16}$ In a recent single-cell RNA seq study of atherosclerotic macrophages, Dnase1L3 was 
present in progressing, but not regressing plaques. Unexpectedly, DNase1L3 was associated with a proinflammatory interferon response, suggesting a harmful role of DNase1 1 3 in athersclerosis ${ }^{17}$, instead of the anti-inflammatory role via clearing NETs. However, a study by Jiménez-Alcázar et al. using DNase $1^{-/-}$and DNase $1 \mathrm{~L}^{- \text {- }}$ single as well as double KO (DKO) mice showed that only the double-deficiency led to vascular occlusion while both single $\mathrm{KO}$ were able to prevent it. ${ }^{18}$ This was in line with increased NET formation in the double compared to the single KO, indicating a detrimental role for NETs in atherothrombotic disease and further, shows that DNase1 as well as DNase1L3 can clear NETs.

Current treatment of atherosclerosis involves lipid-lowering therapy, but is only preventing a fraction of recurrent events in survivors of myocardial infarction..$^{19,20}$ It is thought that inflammation resolution is needed to get the most benefit of lipid-lowering therapies. ${ }^{21,22}$ Hence, degradation of NETs using endonucleases is a potential therapeutic target to decrease inflammation and thereby overcome the residual risk of CVD after lipid-lowering therapy. DNase1 is clinically already used to treat cystic fibrosis ${ }^{23}$ and shows no toxicity, and thus represents a possible therapeutic approach. However, as noted above, current published results on DNases in atherosclerosis are controversial and still scant, and further research is needed to elucidate the role of the two endonucleases in atherosclerosis.

To assess which endonuclease plays the main role in clearing NETs in atherosclerotic plaques, we used three different knock-outs - DNase1, DNase1L3 and a DKO. We hypothesized that knocking out either endonuclease would increase NET formation, with the most prominent effect in the double-deficient mice, and thereby enhance atherosclerosis progression and impair regression. Surprisingly, we did not find differences in NET content between the sufficient and single-deficiency models, but detected a trend towards increased NET formation in the double-deficient model. In line with no significant differences in NET content, loss of DNase1, DNase1L3 or both did not lead to increased progression or impaired regression measured as plaque area and macrophage content. Notably, NET formation was limited likely due to rather low cholesterol levels leading to insignificant results. These outcomes suggest that a stronger or an additional stimulus, such as higher cholesterol levels than we achieved or hyperglycemia, is needed for enhanced NET formation leading to adverse effects on atherosclerosis. Nonetheless, this pilot-study gives first hints on the role of different 
endonucleases in atherosclerosis and an increase in $\mathrm{n}$ is needed to draw final conclusions.

\section{Materials and Methods}

Animals and Atherosclerosis Studies: The Dnase $1 \mathrm{~L}^{-/-}$mouse model on C57BL/6 (B6) background [Dnase1L3 ${ }^{\text {LacZ/LacZ }]}$ has been described previously. ${ }^{24}$ Mice carrying a targeted allele of Dnase1 (Dnase 1m1.1(KOMP)VIcg) on B6 background were obtained from the Knockout Mouse Project and crossed with WT B6 and Dnase $1 \mathrm{~L}^{\mathrm{O}^{-/}}$mice to obtain Dnase $1^{-/-}$and Dnase $1^{-/-} / D n a s e 1 L 3^{-/-}$mice, respectively. C57BL/6J (WT; Jackson Laboratory, stock No: 000664).

Mice of both gender were injected with a gain of function mutant of proprotein convertase subtilisin/kexin type 9 (PCSK9)-encoding adeno-associated vector (AAVmPCSK9; AAV.8TBGmPCSK9D377Y) as done before ${ }^{25}$ and fed a western diet (WD; 0.3\% cholesterol, Dyets Inc. D101977Gi) for 20 weeks (progression). To induce regression, mice were injected with apolipoprotein $B$ antisense oligo nucleotides (ApoB ASO, Ionis Pharmaceuticals) and fed a chow diet for 4 weeks. AAV PCSK9 was injected $1 \mathrm{x}$ at a concentration of $1 * 10^{12}$ viral particles/mouse. ApoB ASO was injected $1 \mathrm{x}$ at a concentration of $50 \mu \mathrm{g} / \mathrm{g}$ body weight (BW).

At day of sacrifice, mice were anesthetized via intraperitoneal (i.p.) injection of ketamine $(100 \mathrm{mg} / \mathrm{kg})$ and xylazine $(10 \mathrm{mg} / \mathrm{kg})$. Blood was collected via cardiac puncture in EDTA-containing tubes and mice perfused with $10 \%$ sucrose in saline solution $(0.9 \% \mathrm{NaCl})$. Collected aortic roots were embedded in optimal cutting temperature $(\mathrm{OCT})$ compound and frozen at $-80^{\circ} \mathrm{C}$.

Plasma Lipid Measurement: Total Cholesterol was measured using Total Cholesterol E Kit (Wako Life Science, cat \# NC9138103).

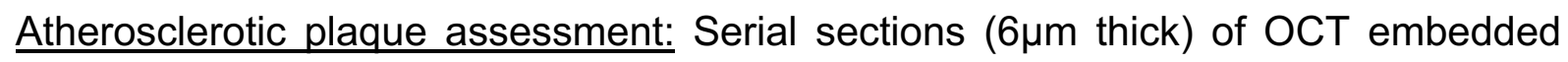
roots were stained for CD68 (rat anti-mouse CD68, Bio-Rad, Cat. MCA1957) as done before $^{26}$. Briefly, sections were fixed and permeabilized with $100 \%$ ice-cold acetone, blocked, and stained with anti-CD68 antibody followed by an incubation with biotinylated anti-rat IgG secondary antibody (Vector Laboratories, CA), and visualized using the Vectastain ABC kit (Vector Laboratories). Slides were counterstained with 
hematoxylin/eosin (Sigma), dehydrated in an ethanol gradient and xylene (Fisher Scientific, NH), and mounted with coverslips using Permount (Fisher Scientific). NETs were identified using colocalization immunofluorescence staining of Ly6G (BD Bioscience, Cat.551459), MPO (Abcam, Cat. ab90812), and citrullinated H3 (H3Cit; Abcam, Cat. ab5103). Tissue was fixed in Formalin, permeabilized using $0.5 \%$ Triton-X, followed by incubation of primary $A b$ o/n at $4^{\circ} \mathrm{C}$ and stained with the secondary antibodies for $2 \mathrm{~h}$ (Thermo Fisher Scientific, Cat. A21434 \& A21245). Stained lesions were imaged (Nikon Eclipse) and lesion area and $\mathrm{CD}^{2} 8^{+}$areas determined using ImagePro Plus software (Micro Optical Solutions). NETs staining was imaged using Hamatsu NanoZoomer 2.0 and $\mathrm{NET}^{+}$area determined using Image J $1.51 \mathrm{r}$.

Statistics: Data are expressed as mean \pm SEM. Data were tested on normality and equal variance and analyzed by appropriate parametric or non-parametric test as stated in each figure, $P \leq 0.05$ was considered significant (GraphPad Prism 7).

No gender differences were observed.

\section{Results and Discussion}

Loss of DNase1, DNase1L3 or both does not significantly change atherosclerotic NET content

DNase 1 has been shown to efficiently clear NETs and its activity inversely associates with SLE severity ${ }^{9-11}$, but how DNases affect atherosclerosis still needs to be determined. DNase 1//DNase1 $13^{-1-}$ mice have been shown to have a higher NET content compared to single-deficient models in atherothrombosis ${ }^{18}$, but no data on atherosclerosis are available. To address this gap, we used WT (control), DNase $1^{1 /}$, DNase $1 \mathrm{~L}^{-/}$, and DNase ${ }^{1-/ D N a s e} 1 \mathrm{~L}^{-/-}$mice injected with $10^{12}$ viral particles/mouse PCSK9 to increase plasma cholesterol levels (Study Setup: Figure 1A). After an atherosclerosis progression period for 20 weeks on western diet (WD, 0.3\% cholesterol), we induced atherosclerosis regression by injecting $50 \mu \mathrm{g} / \mathrm{g}$ (of body weight; BW) apoB ASO and placed the mice on chow diet for 4 weeks. BW did not differ between groups and compared to control, neither during progression or regression (Figure 1B, Supplemental Table1). Cholesterol levels during atherosclerosis progression were lower in the DNase $1 \mathrm{~L}^{-/-}$mice $(468.2 \pm 39.61 \mathrm{mg} / \mathrm{dL})$ 
compared to the other groups (WT $638.2 \pm 51.5$, DNase1/ $721.3 \pm 87.2$, DNase ${ }^{-/}$ $/ D N a s e 1 \mathrm{~L}^{-/-} 618.1 \pm 64.8 ; \mathrm{mg} / \mathrm{dL}$ ). We saw the same pattern during regression (WT

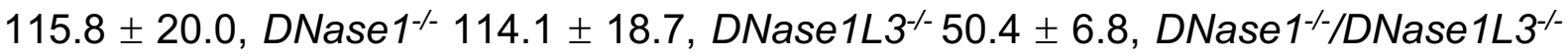
$101.8 \pm 11.7 ; \mathrm{mg} / \mathrm{dL})$.

To evaluate NET content, we performed NETs staining (MPO, Ly6G, H3Cit; Figure 1D) and quantified NETs as \% of total plaque area (Figure 1E). NETs did not significantly differ between groups, neither during progression or regression. DNase1L3 deficient mice did not develop NETs during progression, while DNase1 deficient mice $(0.7 \pm 0.3 \%$ plaque area) as well as the double-deficient mice $(3.3 \pm 1.4$ $\%$ plaque area) did. At first glance, these results could be interpreted in the sense that DNase1 dominates over DNase1L3 in clearing NETs and that DNase1L3 might even promote NET formation. However, control mice expressing both endonucleases DNase1 and DNase1L3 - had the same amount of NETs as DNase1 deficient mice. With DNase1 clearing NETs and DNase1L3 promoting NETs, we would have expected to see decreased NET formation in control compared to DNase1 deficient mice. Further, the trend towards increased NET formation in the double-deficient model compared to control $(3.3 \pm 1.4$ versus $1.5 \pm 1.0 \%$ plaque area), suggests that both DNases can clear NETs. This is in contrast to DNase1L3 promoting NET formation and would assume NET formation to be higher than 0 in DNase $1 \mathrm{~L}^{-/-}$mice. Overall, interpretation of the data should be done with caution, as our study has rather low cholesterol levels (WT $\sim 600 \mathrm{mg} / \mathrm{dL})$, especially in the DNase $1 \mathrm{~L}^{-/}$mice $(\sim 470 \mathrm{mg} / \mathrm{dL})$. This was unexpected since we did observe higher cholesterol levels $(\sim 1200 \mathrm{mg} / \mathrm{dL})$ using PCSK9 before ${ }^{25}$, and could be the result of a change in animal facility and staff. Therefore, the lack of NETs in DNase $1 \mathrm{~L}^{-/-}$mice could be due the relatively low cholesterol levels rather than the higher efficiency of DNase1 or DNase1L3 promoting NETs. The less severe mouse model might have also led to not being able to recapitulate the NETs resolving effect during regression as shown before using $\mathrm{LdIr}^{\mathrm{L}}$ mice on WD for 16 weeks. The NET content after the progression period in those two studies differed by $\sim 4$-fold (WT + AAV PCSK9 20wks WD: 1.4\%plaque area; LdIr/16wks WD: 5.7\%plaque area). Hence, NET formation was likely not high enough to measure differences in NET content between progression and regression. In contrast, another study showed NET formation $<1 \%$ in $L d L r^{-}$mice after 12 weeks on WD 
despite cholesterol levels $\sim 1000 \mathrm{mg} / \mathrm{dL}$, suggesting that NETs formation is independent of cholesterol levels. ${ }^{27}$

Our results propose that neither endonuclease - DNase1 nor DNase1L3 dominates over the other in clearing NETs in atherosclerotic plaques. Further, enhanced NET formation and stronger differences might have been observed with a more severe atherosclerosis model and increased $n$ numbers are necessary for definitive determination.

A
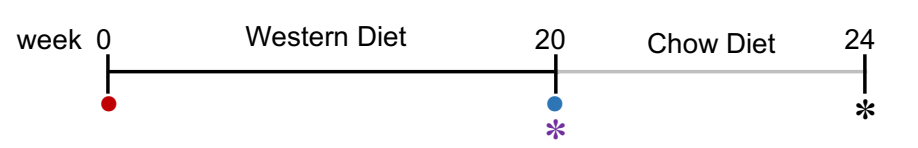

- PCSK9

- ApoB ASO

* Harvest Progression

* Harvest Regression

B
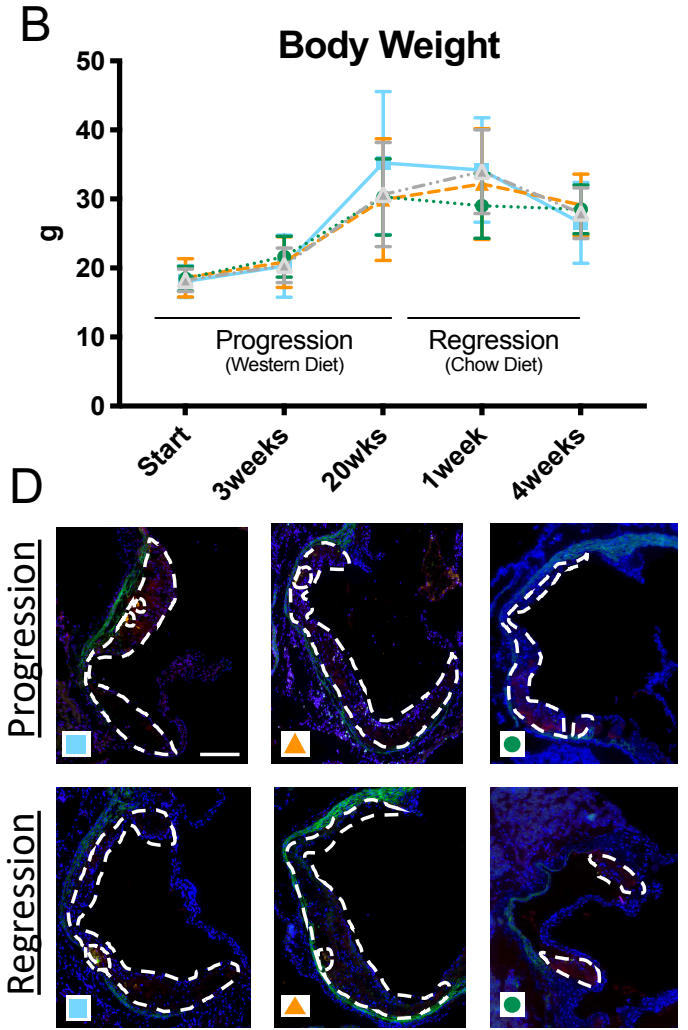

C

$$
\begin{aligned}
& \text { Plasma Cholesterol } \\
& 1500
\end{aligned}
$$

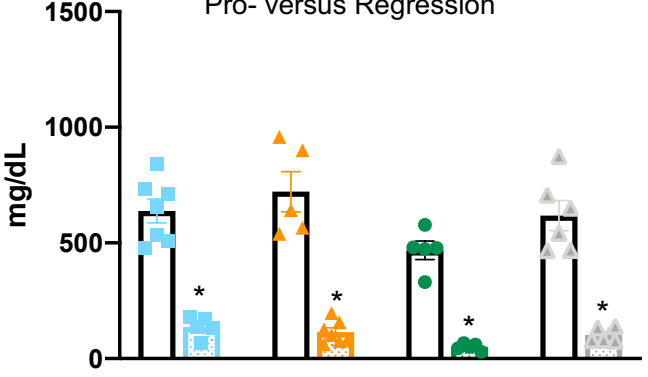

$E$

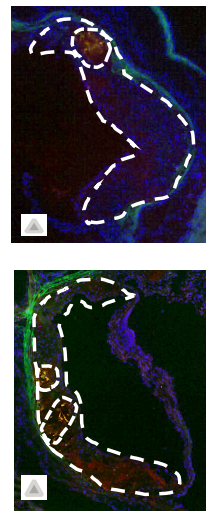

\%NETs

Pro- versus Regression

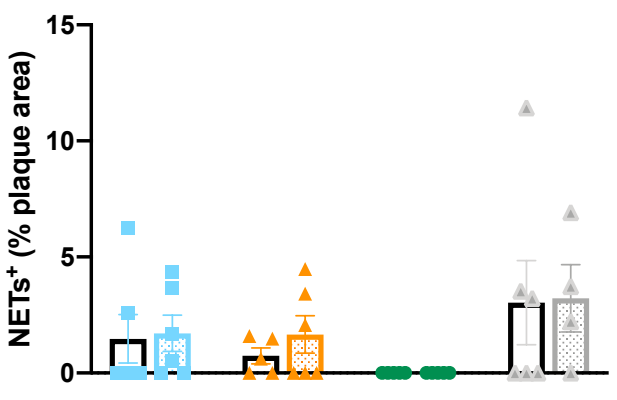

WT $\triangle$ DNase1\% ODNase1L3\% $\triangle$ DNase1\%/DNase1L3\%

Figure 1. Loss of DNase1, DNase1L3 or both does not significantly change atherosclerotic NET content

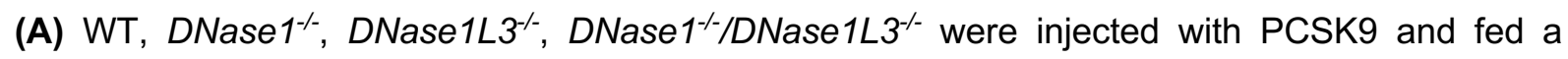
western diet for 20 weeks to develop plaques. At week 20, a subset of mice was harvested for the progression group, all other mice received $1 \mathrm{x}$ ApoB ASO injection and were fed a chow diet for 4 weeks to induce plaque regression. (B) Body weight (g) and (C) Plasma Cholesterol Levels (mg/dL) at time of harvest. (D) NETs staining (scale bar $100 \mu \mathrm{m}$ ) and (E) quantification in aortic roots as 
determined by composite staining of myeloperoxidase (MPO), Ly6G and citrullinated Histone 3 (H3Cit). Data is presented as mean $\pm \mathrm{SEM}, \mathrm{N}=4$-6/group ${ }^{*} \mathrm{P}<0.05,1$-way ANOVA with Tukey's multiple comparison test (C), Kruskal-Wallis test (E).

\section{Loss of DNase1, DNase1L3 or both does not affect atherosclerosis}

To determine if NET content and the slight increase in the DKO mice had an impact on atherosclerosis, we measured plaque area $\left(\mu \mathrm{m}^{2}\right.$, Figure $\left.2 \mathrm{~A}-\mathrm{B}\right)$ and macrophage content represented as percentage of plaque area (\%CD68; Figure 2C). Plaque area was similar in each group during progression and did not decrease during regression (Figure 2B). Plaque area in DNase $1 \mathrm{~L}^{-/-}$mice was significantly lower compared to control and DNase1- mice, likely due to lower cholesterol levels (Figure 1C). As expected, macrophage content (\%CD68) significantly decreased during atherosclerosis regression (Figure $2 \mathrm{C}$ ). We did not observe significant differences between groups, but a trend towards impaired regression in the DKO ( 49\% reduction) compared to the single KO ( 60\% DNase $1^{--}, \sim 80 \%$ DNase $\left.1 \mathrm{~L}^{--/}\right)$, which is in line with NET content. However, the control group also showed a $\sim 44 \%$ reduction in macrophage content and we did not observe a correlation between \%CD68 and NETs (data not shown). 
A CD68 $\mapsto 100 \mu m$

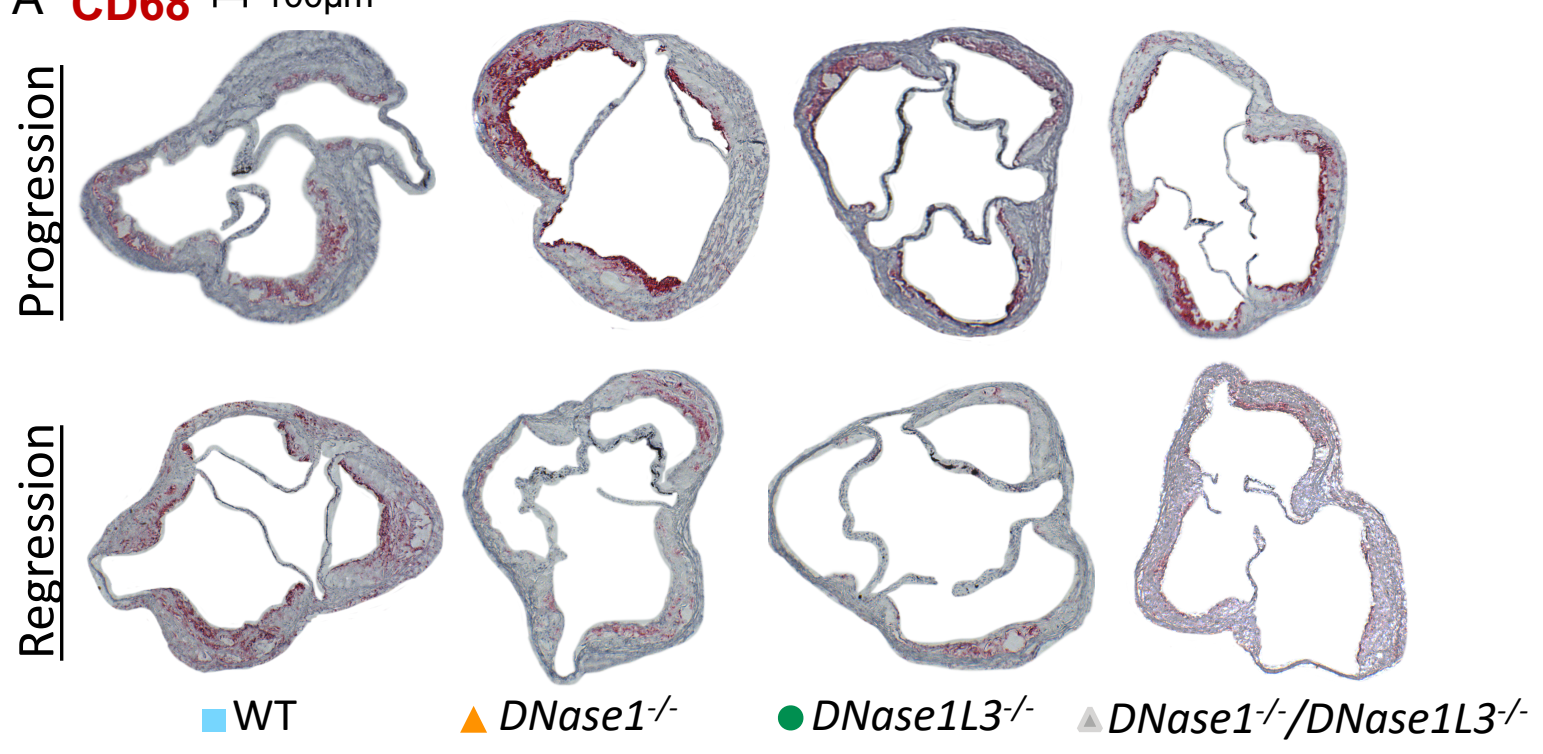

B

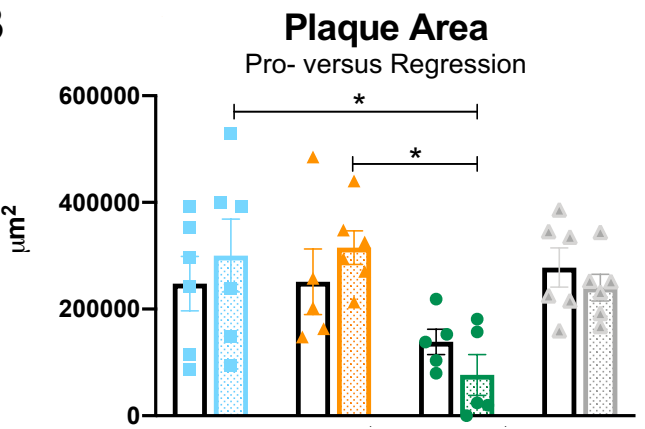

C

$\%$ CD68

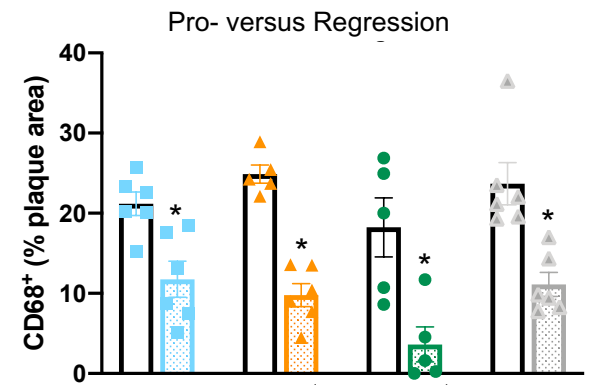

Figure 2. Loss of DNase1, DNase1L3 or both does not affect atherosclerosis

(A) Representative pictures and quantification of (B) plaque area $\left(\mu \mathrm{m}^{2}\right)$ and (C) $\mathrm{CD}^{+} 8^{+}$area (\%plaque area). Scale bar $100 \mu \mathrm{m}$. Data is shown as mean \pm SEM, $N=5-6 /$ group ${ }^{*} \mathrm{P}<0.05$, 1 -way ANOVA with Tukey's multiple comparison test.

In conclusion, this pilot study shows that loss of DNase1, DNase1L3 or both does not significantly change NET content, worsen atherosclerosis progression or impair atherosclerosis regression. The trend towards higher NET formation in the doubledeficient model suggests that with a stronger stimulus, atherosclerosis will be more likely affected compared to the fully sufficient and single-deficient models. To confirm the results of our study, $\mathrm{n}$ number should be increased. Further, adding an additional stimulus, such as hyperglycemia, which is known to increase NET formation and impair atherosclerosis regression, is of interest. 


\section{References}

1 Takeshita, H. et al. Mouse deoxyribonuclease I (DNase I): biochemical and immunological characterization, cDNA structure and tissue distribution. Biochem Mol Biol Int 42, 65-75, doi:10.1080/15216549700202441 (1997).

2 Liu, Q. Y. et al. DNaseY: a rat DNaseI-like gene coding for a constitutively expressed chromatin-bound endonuclease. Biochemistry 37, 10134-10143, doi:10.1021/bi9800597 (1998).

3 Keyel, P. A. Dnases in health and disease. Dev Biol 429, 1-11, doi:10.1016/j.ydbio.2017.06.028 (2017).

4 Brinkmann, V. et al. Neutrophil extracellular traps kill bacteria. Science 303, 1532-1535, doi:10.1126/science.1092385 (2004).

5 Fuchs, T. A. et al. Novel cell death program leads to neutrophil extracellular traps. J Cell Biol 176, 231-241, doi:10.1083/jcb.200606027 (2007).

6 Awasthi, D. et al. Oxidized LDL induced extracellular trap formation in human neutrophils via TLR-PKC-IRAK-MAPK and NADPH-oxidase activation. Free Radic Biol Med 93, 190-203, doi:10.1016/j.freeradbiomed.2016.01.004 (2016).

7 Khandpur, R. et al. NETs are a source of citrullinated autoantigens and stimulate inflammatory responses in rheumatoid arthritis. Sci Transl Med 5, 178ra140, doi:10.1126/scitranslmed.3005580 (2013).

8 Lazzaretto, B. \& Fadeel, B. Intra- and Extracellular Degradation of Neutrophil Extracellular Traps by Macrophages and Dendritic Cells. J Immunol 203, 22762290, doi:10.4049/jimmunol.1800159 (2019).

9 Hakkim, A. et al. Impairment of neutrophil extracellular trap degradation is associated with lupus nephritis. Proc Natl Acad Sci U S A 107, 9813-9818, doi:10.1073/pnas.0909927107 (2010).

10 Yasutomo, K. et al. Mutation of DNASE1 in people with systemic lupus erythematosus. Nat Genet 28, 313-314, doi:10.1038/91070 (2001).

11 Chitrabamrung, S., Rubin, R. L. \& Tan, E. M. Serum deoxyribonuclease I and clinical activity in systemic lupus erythematosus. Rheumatol Int 1, 55-60, doi:10.1007/bf00541153 (1981).

12 Soehnlein, O. Multiple roles for neutrophils in atherosclerosis. Circ Res 110, 875888, doi:10.1161/CIRCRESAHA.111.257535 (2012).

13 Doring, Y., Soehnlein, O. \& Weber, C. Neutrophil Extracellular Traps in Atherosclerosis and Atherothrombosis. Circ Res 120, 736-743, doi:10.1161/CIRCRESAHA.116.309692 (2017).

14 Warnatsch, A., Ioannou, M., Wang, Q. \& Papayannopoulos, V. Inflammation. Neutrophil extracellular traps license macrophages for cytokine production in atherosclerosis. Science 349, 316-320, doi:10.1126/science.aaa8064 (2015).

15 Yamamoto, K. et al. Augmented neutrophil extracellular traps formation promotes atherosclerosis development in socially defeated apoE(-/-) mice. Biochem Biophys Res Commun 500, 490-496, doi:10.1016/j.bbrc.2018.04.115 (2018).

16 Soehnlein, O., Ortega-Gomez, A., Doring, Y. \& Weber, C. Neutrophil-macrophage interplay in atherosclerosis: protease-mediated cytokine processing versus NET release. Thromb Haemost 114, 866-867, doi:10.1160/TH15-08-0623 (2015).

17 Lin, J. D. et al. Single-cell analysis of fate-mapped macrophages reveals heterogeneity, including stem-like properties, during atherosclerosis progression and regression. JCI Insight 4, doi:10.1172/jci.insight.124574 (2019). 
18 Jimenez-Alcazar, M. et al. Host DNases prevent vascular occlusion by neutrophil extracellular traps. Science 358, 1202-1206, doi:10.1126/science.aam8897 (2017).

19 Libby, P. The forgotten majority: unfinished business in cardiovascular risk reduction. J Am Coll Cardiol 46, 1225-1228, doi:10.1016/j.jacc.2005.07.006 (2005).

20 Masoudi, F. A. et al. Trends in U.S. Cardiovascular Care: 2016 Report From 4 ACC National Cardiovascular Data Registries. J Am Coll Cardiol 69, 1427-1450, doi:10.1016/j.jacc.2016.12.005 (2017).

21 Ridker, P. M. et al. Antiinflammatory Therapy with Canakinumab for Atherosclerotic Disease. N Engl J Med 377, 1119-1131, doi:10.1056/NEJMoa1707914 (2017).

22 Tardif, J. C. et al. Efficacy and Safety of Low-Dose Colchicine after Myocardial Infarction. N Engl J Med, doi:10.1056/NEJMoa1912388 (2019).

23 Mogayzel, P. J., Jr. et al. Cystic fibrosis pulmonary guidelines. Chronic medications for maintenance of lung health. Am J Respir Crit Care Med 187, 680-689, doi:10.1164/rccm.201207-1160oe (2013).

24 Sisirak, V. et al. Digestion of Chromatin in Apoptotic Cell Microparticles Prevents Autoimmunity. Cell 166, 88-101, doi:10.1016/j.cell.2016.05.034 (2016).

25 Peled, M. et al. A wild-type mouse-based model for the regression of inflammation in atherosclerosis. PLoS One 12, e0173975, doi:10.1371/journal.pone.0173975 (2017).

26 Rahman, K. et al. Inflammatory Ly6Chi monocytes and their conversion to M2 macrophages drive atherosclerosis regression. J Clin Invest 127, 2904-2915, doi:10.1172/JCI75005 (2017).

27 Westerterp, M. et al. Cholesterol Efflux Pathways Suppress Inflammasome Activation, NETosis, and Atherogenesis. Circulation 138, 898-912, doi:10.1161/CIRCULATIONAHA.117.032636 (2018). 


\section{Supplemental}

Supplemental Table I. Body weight (g) at harvest

\begin{tabular}{|c|c|c|c|c|}
\hline & WT & DNase1\%- & DNase1L3\%- & DNase1/-/DNase1L3-/- \\
\hline Progression & $30.5 \pm 4.8$ & $25.3 \pm 0.7$ & $31.0 \pm 2.3$ & $27.7 \pm 2.0$ \\
\hline Regression & $28.8 \pm 2.5$ & $29.2 \pm 1.8$ & $28.5 \pm 1.6$ & $27.9 \pm 1.5$ \\
\hline
\end{tabular}


Summary \& Discussion 
The main goal of this thesis was to investigate two different aspects of the 2 to 4 -fold increased CVD risk in individuals with diabetes. Diabetes is an independent CVD risk factor, even after lipid-lowering therapy and further, impairs regression of atherosclerosis. A study by Nicholls et al in 2008 showed that lowering LDL-C to desired levels of $<80 \mathrm{mg} / \mathrm{dL}$ in individuals with no diabetes promotes atherosclerosis regression in almost $25 \%$ of them, measured as reduction in lesion size by intravascular ultrasound (Figure 1). ${ }^{1}$ In individuals with diabetes, lowering LDL-C levels to $<80 \mathrm{mg} / \mathrm{dL}$ did only promote regression in $\sim 17 \%$ individuals. Strikingly, the number of individuals with regression in non-diabetics with elevated plasma LDL-C and diabetics with desired LDL-C is similar. Our lab has been able to replicate findings consistent with these in mice, showing that diabetic mice have about $50 \%$ the regression of non-diabetic mice. ${ }^{2}$
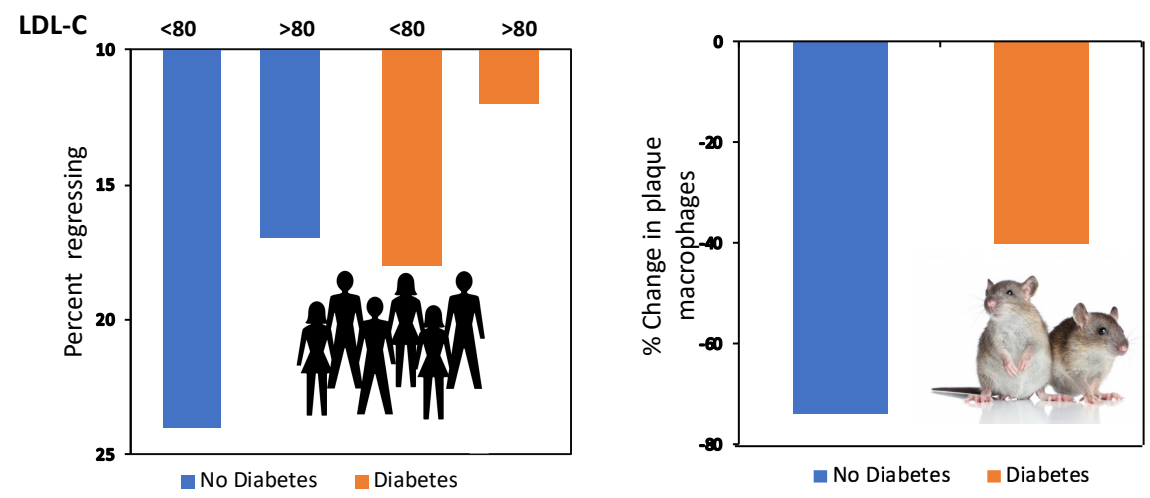

Figure 1. Comparison of atherosclerosis regression with and without diabetes in humans and mice. Human data (left) are based on the results published by Nicholls et al. ${ }^{1}$ Mouse data (right) are based on our published study by Parathath et al. ${ }^{2}$

In this thesis, two probable causes for residual CVD risk, which are involved in high risk populations such as patients with diabetes, have been discussed:

- Effect of elevated plasma TG levels and low HDL (Chapters 2-4)

- Effect of hyperglycemia-associated circulating neutrophils and NET formation (Chapters 5 \& 6) 


\section{Triglycerides and their role in atherosclerosis}

Main findings

Chapter 2 describes an inverse association of HDL characteristics, such as HDL-C, apo-AI, HDL size and HDL-P, with pre-clinical atherosclerosis and CVD/CVE in a human cohort. Surprisingly, CEC was not associated with atherosclerosis surrogates or CVD/CVE. Stratifying the population in subjects with NGM and (pre)diabetes, revealed that associations of HDL-size and HDL-P with prevalence of CVD are lost. Chapter 3 shows that the role of $\mathrm{LpL}$ in macrophage polarization in vitro does not resemble the in vivo situation. While LpL regulates lipid uptake, as well as pro- and anti-inflammatory gene expression in macrophages in vitro, myeloid $\mathrm{LpL}$ deficiency in vivo is dispensable for lipid accumulation and macrophage polarization. Global LpL deficiency in vivo reduces lipid content in adipose tissue macrophages (ATM) and the number of induced peritoneal macrophages. Further, global LpL deficiency does affect the phenotype of circulating monocytes and peritoneal macrophages, but does not affect macrophages in regressing atherosclerotic plaques. Chapter 4 reports that LpLinduced HyperTG causes reduced HDL-C and HDL-P in mice and humans, but no dysfunctional HDL measured as CEC. Despite the reduction in HDL-C and HDL-P, LpL-deficiency-induced HyperTG did not impair atherosclerosis regression, neither in aortic arches, roots or BCAs, likely because HDL function (CEC) was maintained.

\section{Discussion}

The connection between low HDL-C and elevated TG with cardiovascular risk has been shown in observational studies, but how HyperTG affects other HDL characteristics is unknown and pre-clinical trials showing benefits of TG reduction are scant. While there are limited experimental models linking HyperTG and atherosclerosis, a large series of experimental studies have documented benefits of raising HDL levels as reviewed in ${ }^{3,4}$. One postulated reason for these benefits is an increase in CEC or reverse cholesterol transport (RCT). ${ }^{5}$ However, interventions in humans that increased HDL-C levels using CETP inhibition did not show clinical benefits $^{6,7}$, indicating that an increase of HDL-C does not necessarily reflect an increase in HDL function (CEC or RCT). In line, although CETP is considered proatherogenic due to its contribution to an atherogenic lipid phenotype, including lowering HDL-C, CETP has also been shown to increase $\mathrm{RCT}^{8}$, which is considered anti- 
atherogenic. We showed that although inducing hCETP expression in mice lowers HDL-C and HDL-P, the ABCA1-mediated efflux per HDL particle was increased. Overall these effects resulted in atherosclerosis regression not being impaired (Chapter 4). Instead of solely measuring HDL-C, focus in the field of research has shifted to determining HDL functions. Therefore, we assessed HDLs' considered key-antiatherogenic function, i.e. CEC, and other characteristics such as apoA-I, HDL-P, and HDL size (Chapter $2 \& 4$ ). As the metabolic milieu may affect various properties of HDL, we looked into how diabetes (Chapter 2) and HyperTG alone (Chapter 4) impact HDL. To further determine how changes in HDL affect CVD, we associated HDL characteristics with pre-clinical atherosclerosis (endothelial dysfunction; EnD) and with CVD/CVE and were the first ones to do so in (pre)diabetic individuals (Chapter 2). Of note, we also associated HDL characteristics with another atherosclerosis surrogate - cIMT - but due to lack of associations, likely due to sensibility of the cIMT measurement itself, these results are not discussed here, but in chapter 2.

The (pre)diabetic individuals of the CODAM Cohort displayed typical features of diabetic dyslipidemia, i.e. reduced HDL-C and increased TG levels. As expected, HDL-C was inversely associated with EnD and CVE in the total cohort and in individuals with (pre)diabetes. Other HDL characteristics, such as apoA-I, HDL size and HDL-P were also inversely associated, with associations of HDL size and HDL-P being lost in individuals with (pre)diabetes, but not in healthy subjects. Notably, while previous studies have reported HDL-P and HDL-size to decrease in diabetes ${ }^{9,10}$, we report an increase of both. With HDL-P and size being increased in (pre)diabetics, but lack of its association with atherosclerosis, this suggests that in individuals with NGM, the protective effect of a certain number of HDL particles against development of atherosclerosis is better than for that same number of HDL particles in (pre)diabetes. To determine if observed associations of HDL characteristics with atherosclerosis and CVE in (pre)diabetics, or the lack thereof, were dependent on TG levels, we performed $T G$ adjusted analyses. While all HDL characteristics were still inversely correlated with atherosclerosis in healthy subjects after adjusting for TG, the association of HDL-C with atherosclerosis was lost specifically in (pre)diabetic patients. This suggests that the inverse relationship of HDL-C with atherosclerosis in (pre)diabetes is dependent on TG thereby confirming the intimate relationship of HDL-C and TG. 
To allow a closer comparison of human data from the CODAM study with human and mouse data from chapter 4 , we stratified the CODAM cohort into $T G<150 \mathrm{mg} / \mathrm{dL}$ ( $\mathrm{N}=363$; control) and $>150 \mathrm{mg} / \mathrm{dL}(\mathrm{N}=167$; HyperTG). Thereby, $66 \%$ of HyperTG patients were (pre)diabetic and had $\mathrm{Hb} 1 \mathrm{Ac}$ levels of $6 \pm 0.9 \%$. Next to reduced HDL-C, HyperTG individuals from the CODAM Cohort also displayed decreased HDL-P, but not change in CEC, compared to control (Figure 2). This is in line with results from chapter 4, showing that despite reduction of HyperTG-induced HDL-C and HDL-P in mice and human, overall CEC is not impaired. Interestingly, individuals with (pre)diabetes have increased HDL-P, but also no change in CEC (Chapter 2). This suggests that $\mathrm{HDL}$ functionality can be preserved despite changes in HDL-P number. Thus, composition of HDL is of particular interest regarding its atheroprotective properties. Nonetheless, if preserved CEC is the reason for not observing impaired regression in HyperTG mice despite a reduction in HDL-C and HDL-P (Chapter 4), we would expect to see an association of CEC with atherosclerosis and CVD/CVE in the CODAM study (Chapter 2) as it has been shown in other studies. ${ }^{11-14}$ So far, CEC measurements are not standardized and although we employed a well validated CEC assay, different protocols could have led to discrepancies between studies. Another limitation is the cross-sectional approach of our study, which hampers a direct causal interpretation of the observed associations. A better comparison of chapter 2 and chapter 4 would have been measuring associations of HDL-characteristics with the change in cIMT over the study period $(\Delta \mathrm{CIMT})$ as surrogate for atherosclerosis regression. However, as mentioned above, we did not find any significant associations of HDL characteristics with cIMT.

HDL-P

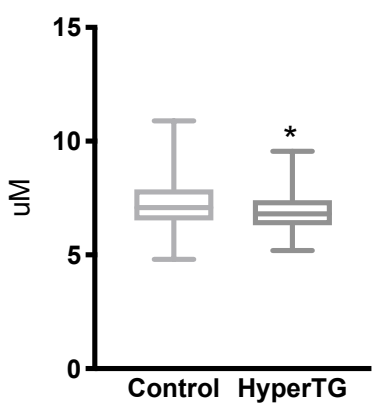

CEC

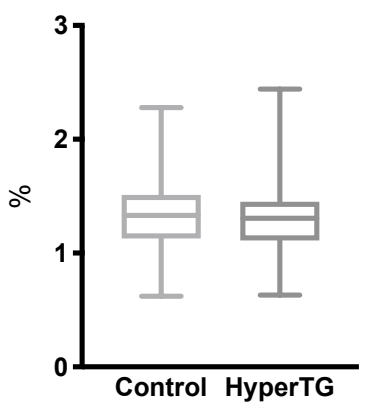

Figure 2. Comparison of HDL-P (left) and CEC (right) between Control ( $\mathrm{TG}<150 \mathrm{mg} / \mathrm{dL}, \mathrm{N}=363$ ) and HyperTG (TG>150mg/dL, $\mathrm{N}=167)$ in the CODAM study. 
HyperTG has been shown to be associated with CVD in a number of studies and is not in line with our results from chapter 4. However, next to CEC, HDL has other functions as well as including antithrombotic functions. ${ }^{15}$ Interestingly, proteomic analysis of HDL revealed an upregulation of proteins that are associated with platelet activation, signaling and degranulation in mice and in humans (data now shown), suggesting a role of HyperTG-reduced HDL in atherothrombosis. Current publications have reported that acute HyperTG induces platelet hyperactivity ${ }^{16}$ and contributes to resistance of antiplatelet therapy such as aspirin and clopidogrel. ${ }^{17,18}$ Additionally, platelet activity was also increased by TG-rich lipoproteins from T2DM patients. ${ }^{19}$ Meta-regression analyses of 64 randomized control trials showed that TG levels were associated with stroke risk, but not cIMT measures. However, TG lowering did only result in a trend towards reduced stroke incidence. ${ }^{20}$ Therefore, the association of HyperTG with increased CVD risk could be explained by loss of HDLs antithrombotic function, but future studies are needed.

Another aspect of the atherogenic potential of HyperTG is the lipolysis of TGs. LpL hydrolyzes TGs either at the vessel wall or within the atherosclerotic plaque, resulting in remnants and other lipolysis products (FFA, mono- diglyceride and lysolecithin). TG lipolysis at the vessel wall leads to atherogenic remnants that can enter the arterial wall and deposit lipids, while lipolysis products (FFA, mono- diglyceride and lysolecithin) can result in increased inflammation, monocyte adhesion and coagulation. In contrast, we did not find impaired vascular regression despite vascular lipolysis (Chapter 4). As macrophage LpL has been implicated in foam cell formation ${ }^{21-23}$, not observing HyperTG-impaired atherosclerosis regression could also be due to counterbalancing effects of Lpl deficiency in macrophages. Thus, macrophage LpL deficiency could prevent lipoprotein uptake by macrophages, masking possible atherogenic effects of HyperTG. Macrophages are the major source of LpL in atherosclerotic plaques ${ }^{24}$ and whether $\mathrm{LpL}$ in atherosclerotic plaques acts pro- or antiatherogenic is still debated. Interestingly, macrophage specific LpL knockout in vivo reduced atherosclerosis. ${ }^{21,22}$ In vitro, macrophage $\mathrm{LpL}$ appears to have atherogenic functions, because it causes accumulation of atherogenic lipoproteins and their rapid uptake by macrophages ${ }^{25}$, which we recapitulated in chapter 3 . It is therefore feasible that LpL deficiency in macrophages overcomes the detrimental 
effects of HyperTG in LpL-deficient mice. However, whether or not LpL was present in macrophages, we did not observe differences in atherosclerosis regression in mice (Chapter 4). The potential antiatherogenic effects of $L p L$ are thought to rely on the fatty acids provided by lipolysis, which were reported to convert macrophages to a more anti-inflammatory phenotype via peroxisome proliferator-activated receptors (PPARs). ${ }^{23,26,27}$ We showed that LpL in vitro changes both, pro- and anti-inflammatory markers, despite a decrease in FFA and increase in glycolysis suggestive of an increase in pro- and decrease of anti-inflammatory markers (Chapter 3). Further, transcriptomic profiling of macrophages in atherosclerosis regressing plaques in vivo did not reveal differences between macrophages expressing $L p L$ and $L p L$ deficient macrophages (Chapters $3 \& 4$ ).

In conclusion, in a human cohort we have shown that HDL-C, but not CEC, is associated with atherosclerosis and CVE, also in patients with diabetes. The found association of atherosclerosis with HDL-P is, however, no longer observed in diabetes patients. Experimentally reducing HDL-C and HDL-P by induction of HyperTG did not impair atherosclerosis regression, likely because CEC was unchanged. Further, lipolysis of circulating TG-enriched lipoproteins at the vessel wall or at atherosclerotic macrophages does not impair regression either. HDL proteins that changed with HyperTG were associated with lipoprotein remodeling (e.g. reduced ApoA-I) and platelet activation, signaling and degranulation. Overall, our results indicate that HyperTG does not impair atherosclerosis regression, but might be of importance for atherothrombosis via decreased antithrombotic functions of HDL and should be addressed in future studies.

\section{Neutrophils and their role in atherosclerosis}

\section{Main Findings}

Chapter 5 shows that NETs lead to inflammasome activation and inflammatory macrophages in atherosclerotic plaques. Our results illustrate that NETs can resolve during atherosclerosis regression. In a hyperglycemic environment however, NETs persisted thereby exacerbating macrophage inflammation and impairing atherosclerosis resolution. DNase1 treatment reduced plaque NETs content and macrophage inflammation, promoting atherosclerosis resolution after lipid-lowering in diabetic mice. Chapter 6 describes a pilot study about the potential role of two different 
endonucleases - DNase1 and DNase1L3 - in atherosclerosis. The data show that double-deficiency tends to increase NET formation compared to single-deficient mice and control with no change in NET content during regression. Further, NET content did not associate with atherosclerosis severity.

\section{Discussion}

The possible implication of neutrophils in the pathophysiology of CVD has long been overshadowed by other leukocyte subtypes, such as monocytes and macrophages, partly due to neutrophil's short life span in the circulation of $<24 \mathrm{~h}$ in mice ${ }^{28}$ and human ${ }^{29-31}$. However, the life span of neutrophils within different tissues as well as under inflammatory conditions is not well defined. Therefore, the discovery of neutrophils ability to form $\mathrm{NETs}^{32}$ and the presence of both in human ${ }^{33-37}$ and mouse ${ }^{38-44}$ atherosclerotic plaques has sparked new interest in neutrophils. It has been shown that inflammation can increase longevity of neutrophils, in part via regulation of anti-apoptotic factors, such as Mcl-1.45,46 While survival of neutrophils could be beneficial in acute inflammation, it might be detrimental in a chronic inflammatory state due to increased NETs release as senescent neutrophils have a higher potential for NET formation. ${ }^{47}$ NETs' proinflammatory role and contribution to chronic inflammatory disease has been postulated to trigger crosstalk of neutrophils with other cells. ${ }^{48,49}$ Warnatsch et al. suggested that the interaction of neutrophils and macrophages leads to inflammasome activation in plaque macrophages and enhanced atherosclerosis progression. ${ }^{38}$ In agreement, using transcriptomic profiling of atherosclerotic macrophages, we prove that macrophages in a NET+ area possess indeed a enhanced proinflammatory phenotype compared to macrophages outside of a NETs area (Chapter 5). Since CVD as well as diabetes are associated with neutrophilia in mice $^{50,51}$ and human ${ }^{52}$ and hyperglycemia has been shown to prime neutrophils for NET production ${ }^{53}$, diabetics are one clinical population in which NETs may be particularly harmful in with respect to cardiovascular risk. Further, plasma NETs markers are not only correlated with severity of CAD, but also with severity of T2DM as judged by correlation of nucleosomes (NETosis marker) with HemoglobinA1c levels (average blood glucose levels over a period of time) in humans. ${ }^{54}$ Studying atherosclerosis regression in diabetic mice, we found impaired atherosclerosis 
regression (Chapter 5), as we have shown before ${ }^{2,55,56}$. Thereby, macrophage content was positively associated with NET content (Chapter 5).

While current studies of NETs focused on atherosclerosis progression, we are the first ones reporting on atherosclerosis regression. Our novel results showed that NETs can spontaneously resolve in a non-hyperlipidemic environment, but not in hyperglycemic conditions, even with non-hyperlipidemia. Thus, presence of NETs could be one reason for the increased CVD risk in diabetic patients, even when they have desired LDL-C levels. One way to clear NETs is treatment with DNase1, as NETs are thought to be mainly degraded by endonucleases ${ }^{57}$ and have been reported to be cleared by DNase1 in atherosclerosis in mice ${ }^{38,58}$. Given published studies and existent clinical use of DNase1 (e.g. cystic fibrosis ${ }^{59}$ ), we treated mice with DNase1. As expected, DNase1 treatment led to reduced NET content and excitingly, promoted atherosclerosis regression despite ongoing hyperglycemia by reducing plaque inflammation (Chapter 5). The pro-inflammatory nature of NETs are thought to increase monocyte and neutrophil recruitment, further enhancing plaque inflammation ${ }^{38,60}$, leading to the hypothesis that DNase1 treatment lowers leukocyte recruitment. We do find increased monocytosis (data not shown) as well as neutrophilia in diabetic mice (Chapter 5), as previously reported by our lab. ${ }^{50,51}$ In line, we also found increased plaque neutrophils in diabetic mice and a trend towards decreased circulating and plaque neutrophil levels upon DNase1 treatment. This is in agreement with a study by Wong et al. showing no difference in neutrophil recruitment in diabetic Padi4\%- (NETs abrogated) mice compared to diabetic WT mice ${ }^{53}$, but in contrast to other studies showing that plaque neutrophil content is reduced upon the ablation of the inflammasome ${ }^{39}$ or NETs abrogation ${ }^{38}$. Notably, not all neutrophils detected in plaques do undergo NETosis, which has been shown before in other in vitro and in vivo studies. ${ }^{61}$ Thus, our results indicate that activation of neutrophils rather than neutrophil levels per se are of relevance for NET formation and its effect on plaque composition.

We have seen that restoring normal circulating cholesterol levels after a period of elevated cholesterol levels, led to spontaneous resolution of NETs without additional DNase1 treatment (Chapter 5), indicating that physiological amounts of endonucleases are sufficient for NETs clearance in healthy conditions. Therefore, we 
were interested to know if DNase 1/- mice have higher NET content in atherosclerotic plaques and if atherosclerosis progression was worse or atherosclerosis regression impaired with DNase1 deficiency. Since it has been shown before that serum from DNase1 KO mice show some residual KO activity that displayed characteristics of DNase1L3 ${ }^{62}$, we decided to include DNase1L3 deficient and double-deficient mice. We found that double-deficient mice showed a trend towards increased NET content, which is in line with a study by Jimenez-Alcazar et al., showing that especially the double-deficient mice have more NETs than single-deficient mice leading to occlusion of vascular vessels. ${ }^{63}$ In contrast to our previous results (Chapter 5), the trend towards increased NET content in double-deficient mice did not result in changes in atherosclerosis progression or regression and further, NETs content did not decrease with regression (Chapter 6). However, we did not observe significant differences in NET content between groups. Further, NET content in progressing mice on WT background was about 4-fold lower compared to NET content we observed in the LDLR-deficient mice and is likely due to circulating plasma cholesterol levels. In our study in chapter 5 using $L d l r^{\prime-}$ fed a WD, we reached cholesterol levels of $\sim 1000 \mathrm{mg} / \mathrm{dL}$. In our study in chapter 6, we switched from Ldlr ${ }^{/-}$to WT mice injected with PCSK9 fed a WD to avoid crossbreeding of DNaseKO mice onto LdIr/background. However, WT mice injected with PCSK9 only reached levels of about $\sim 60 \%$ from those of $\mathrm{Ldll}^{\mathrm{K}}$ mice. We did observe higher cholesterol levels ( $1200 \mathrm{mg} / \mathrm{dL})$ using PCSK9 before ${ }^{64}$ and the rather low cholesterol levels could be the results of a change in animal facility and staff. In line with lower cholesterol levels, we also observed lower atherosclerotic plaque sizes. Hence, low NET content could be due to lack of stimulus. If NET formation requires these high levels of cholesterol and can resolve spontaneously by lowering cholesterol levels, clinical relevance of NETs in hyperlipidemia is questionable, since patients with cholesterol levels of $>200 \mathrm{mg} / \mathrm{dL}$ are already put on lipid-lowering therapy. However, NETs do not resolve under hyperglycemic conditions, not even after lipid-lowering. Hence, population to benefit from DNase1 treatment are patients suffering from diabetes, especially those with not well-controlled blood glucose levels. Clinical use of DNase1 (Pulmozyme ${ }^{\circledR}$ ) has been approved by the FDA (Federal Drug Administration) in 1993 and since then is on the market in $>65$ countries with no signs of major toxicity or detrimental long-term effects. Pulmozyme $^{\circledR}$ has been shown to be effective in treatment of cystic fibrosis by 
increasing lung function and reducing exacerbation of cystic fibrosis. This beneficial effect has been proposed to be partly due to NETs degradation by DNase1. ${ }^{65}$ Thus, DNase1 treatment to lower plaque inflammation and to induce atherosclerosis resolution would be a novel therapeutic approach to overcome increased CVD risk in diabetic patients. We (Chapter 5) and others ${ }^{38,53,58}$ have shown that NETs degradation via DNase1 is beneficial in the context of CVD in mice. Notably, there is one contradictory study showing no effect of DNase1 treatment on atherosclerosis ${ }^{66}$, including the thought that DNase1 has little effect on other NET components, such as neutrophil elastase, that might continue to cause inflammation at the vessel wall. ${ }^{67}$ NETs from isolated human neutrophils can also be degraded by DNase1. ${ }^{68}$ Interestingly, it has been shown that an increase in circulating NETs is counterbalanced by an increase in DNase activity in healthy individuals. ${ }^{69}$ Further, mutations in DNAse1 gene has been associated with disease states such as myocardial infarction ${ }^{70}$ or SLE ${ }^{71,72}$. Thus, DNase1 activity might be impaired in individuals suffering from diabetes. In contrast, one study shows that DNase1 activity is actually increased in diabetes ${ }^{73}$ and further research is needed for clarification.

So far, there are no reports on CVD risk in individuals treated with Pulmozyme ${ }^{\circledR}$, however whether DNase1 reaches atherosclerotic plaques by delivery via a nebulizer is questionable. DNase1 administration in current mouse atherosclerosis studies is done via intraperitoneal injections, but in humans this may compromise immunoprotective functions of NETs e.g. antimicrobial activity. A better way to administrate DNase1 might be DNase1-coated nanoparticles, which were shown to reduce size of lung metastases in mice ${ }^{74}$, but this way of administration needs further exploration in atherosclerosis. Although evidence for the detrimental effects of NETs in several diseases such as diabetes and atherosclerosis is raising, current research is still in its infancies. Further research is needed to deepen the knowledge about NETs in CVD and the exact mechanisms of DNase 1 treatment to translate those findings into a novel therapy strategy.

\section{Future Directions}

Several questions have been raised by the results of this thesis and need to be addressed in future studies. 
The effect modifications by the insulin resistant state in the CODAM study are highly interesting and are worth to further investigate in a larger cohort with sufficient power for more extensive subgroup analyses.

Interestingly, HDL proteomics revealed increased platelet associated markers on TG-enriched HDL and future experiments should focus on the role of HyperTG in thrombosis. Therefore, platelet aggregation assays using platelet-rich plasma of HyperTG patients and LpL deficient patients should be tested against control plasma to see if published results of HyperTG inducing platelet hyperactivity can be replicated. If that is the case, aggregation assays should be repeated using incubation of isolated HDL from healthy and HyperTG (LpL deficient) subjects. To determine the effect on atherothrombosis, experimental studies should be performed using a mouse model of thrombosis such as ligation of the inferior vena cava. ${ }^{75}$ Aggregation assays should also be done using platelet-rich plasma from $L p L$ deficient and control mice and further, flow cytometry assays using platelet markers (e.g. P-selectin, JON/A) can give additional insights on platelet activation.

Our novel results showed the therapeutic potential of NET reduction in individuals with diabetes, especially in those whose diabetes is not well controlled. Future studies should focus on understanding the mechanisms by which DNase1 leads to improved atherosclerosis regression in a hyperglycemic condition. We have shown that DNase1 treatment reduced inflammation in atherosclerotic plaques, thereby improving atherosclerosis regression. Additional inflammatory markers in the circulation are of interest. Since we were not able to detect IL $1 \beta$ in the circulation using ELISA and CBA assays, other markers, such as IL-17, IL-18, IL-6, can be tested. Decreased inflammation and improved atherosclerosis regression also lead to the hypothesis that fewer monocytes were recruited, macrophage retention is improved and/or proliferation is reduced. To test this, monocyte and macrophage trafficking studies should be performed. Preliminary results showed reduced circulating Ly6Chigh monocytes with DNase 1 treatment, but no differences in monocyte trafficking (data not shown) and completed study results need to be waited for to draw final conclusions. Another aspect to consider is the possible effect of hypercholesterolemia and/or hyperglycemia on DNase1 activity. NETs have been shown to be involved in the pathogenesis of systemic lupus erythematosus (SLE) ${ }^{68}$, partly due to reduced DNase1 
activity $^{71,76}$. This raises the question if hypercholesterolemia/hyperglycemia may have similar effects, hence the increased NET formation and inflammation. In contrast, one study reported increased DNase1 activity in serum of individuals with diabetes and of STZ-injected rats. $^{73}$ For clarification, DNase activity of hypercholesterolemic and hyperglycemic mice should be compared to control and DNase1 treated mice.

As physiological amounts of endonucleases are sufficient in clearing NETs and results of DNase deficient mouse models were modest under hypercholesterolemic conditions, future studies should focus on hyperglycemia. Given that we see positive effects on DNase1 treatment in diabetic mice and increased NET formation with the double-deficient mouse model, the impact of double-deficient diabetic mice on atherosclerosis is of interest.

\section{Main conclusion \& clinical recommendation}

HyperTG and hyperglycemia have similar effects on HDL, but while HyperTG alone did not impair regression, hyperglycemia does. We observed reduced HDL-C (HyperTG and (pre)diabetics) and HDL-P (HyperTG only), but no differences in CEC (HyperTG and (pre)diabetes) in mice and humans. Experimental studies showed no impairment of atherosclerosis regression with HyperTG, but with hyperglycemia. Thereby, circulating neutrophil levels were increased along with NET content in atherosclerotic plaques and macrophage inflammation. Of note, we did not observe NETs in HyperTG mice (data not shown). Excitingly, clearing NETs using DNase1 lowers plaque inflammation and can overcome diabetes-impaired regression, despite ongoing hyperglycemia.

These results suggest that treating hyperglycemia-associated inflammation is prominent over treating HyperTG in the residual CVD risk in individuals with diabetes. Future studies should focus on establishing the mechanisms of DNase1 treatment and on evaluating the possible effect of HyperTG on other HDL characteristics such as its antithrombotic function. 


\section{References}

1 Nicholls, S. J. et al. Effect of diabetes on progression of coronary atherosclerosis and arterial remodeling: a pooled analysis of 5 intravascular ultrasound trials. $J$ Am Coll Cardiol 52, 255-262, doi:10.1016/j.jacc.2008.03.051 (2008).

2 Parathath, S. et al. Diabetes adversely affects macrophages during atherosclerotic plaque regression in mice. Diabetes 60,1759-1769, doi:10.2337/db10-0778 (2011).

3 Feig, J. E. et al. HDL promotes rapid atherosclerosis regression in mice and alters inflammatory properties of plaque monocyte-derived cells. Proc Natl Acad Sci U S $A$ 108, 7166-7171, doi:10.1073/pnas.1016086108 (2011).

4 Hewing, B. et al. Effects of native and myeloperoxidase-modified apolipoprotein a-I on reverse cholesterol transport and atherosclerosis in mice. Arterioscler Thromb Vasc Biol 34, 779-789, doi:10.1161/ATVBAHA.113.303044 (2014).

5 Rohatgi, A. et al. in New England Journal of Medicine Vol. 371 2383-2393 (2014).

6 Barter, P. J. et al. in New England Journal of Medicine Vol. 357 2109-2122 (2017).

7 Group, H.-T. C. in New England Journal of Medicine Vol. 371 203-212 (2014).

8 Barter, P. J. et al. in The Lancet Vol. 23 160-167 (2003).

9 Mora, S. et al. Lipoprotein particle size and concentration by nuclear magnetic resonance and incident type 2 diabetes in women. Diabetes 59, 1153-1160, doi:10.2337/db09-1114 (2010).

10 Festa, A. et al. Nuclear magnetic resonance lipoprotein abnormalities in prediabetic subjects in the Insulin Resistance Atherosclerosis Study. Circulation 111, 3465-3472, doi:10.1161/CIRCULATIONAHA.104.512079 (2005).

11 Khera, A. V. et al. Cholesterol efflux capacity, high-density lipoprotein function, and atherosclerosis. $N$ Engl J Med 364, 127-135, doi:10.1056/NEJMoa1001689 (2011).

12 Rohatgi, A. et al. HDL cholesterol efflux capacity and incident cardiovascular events. N Engl J Med 371, 2383-2393, doi:10.1056/NEJMoa1409065 (2014).

13 Saleheen, D. et al. Association of HDL cholesterol efflux capacity with incident coronary heart disease events: a prospective case-control study. Lancet Diabetes Endocrinol 3, 507-513, doi:10.1016/S2213-8587(15)00126-6 (2015).

14 Shea, S. et al. Cholesterol Mass Efflux Capacity, Incident Cardiovascular Disease, and Progression of Carotid Plaque. Arterioscler Thromb Vasc Biol 39, 89-96, doi:10.1161/ATVBAHA.118.311366 (2019).

15 Mineo, C., Deguchi, H., Griffin, J. H. \& Shaul, P. W. Endothelial and antithrombotic actions of HDL. Circ Res 98, 1352-1364, doi:10.1161/01.RES.0000225982.01988.93 (2006).

16 Aye, M. M. et al. Acute hypertriglyceridemia induces platelet hyperactivity that is not attenuated by insulin in polycystic ovary syndrome.J Am Heart Assoc 3, e000706, doi:10.1161/JAHA.113.000706 (2014).

17 Jastrzebska, M. et al. Variable gender-dependent platelet responses to combined antiplatelet therapy in patients with stable coronary-artery disease.J Physiol Pharmacol 69, doi:10.26402/jpp.2018.4.10 (2018).

18 Karepov, V., Tolpina, G., Kuliczkowski, W. \& Serebruany, V. Plasma triglycerides as predictors of platelet responsiveness to aspirin in patients after first ischemic stroke. Cerebrovasc Dis 26, 272-276, doi:10.1159/000147455 (2008). 
19 Boulet, M. M. et al. Large triglyceride-rich lipoproteins from fasting patients with type 2 diabetes activate platelets. Diabetes Metab, doi:10.1016/j.diabet.2019.03.002 (2019).

20 Labreuche, J., Deplanque, D., Touboul, P. J., Bruckert, E. \& Amarenco, P. Association between change in plasma triglyceride levels and risk of stroke and carotid atherosclerosis: systematic review and meta-regression analysis. Atherosclerosis 212, 9-15, doi:10.1016/j.atherosclerosis.2010.02.011 (2010).

21 Babaev, V. R. et al. Macrophage lipoprotein lipase promotes foam cell formation and atherosclerosis in vivo. J Clin Invest 103, 1697-1705, doi:10.1172/JCI6117 (1999).

22 Van Eck, M., Zimmermann, R., Groot, P. H., Zechner, R. \& Van Berkel, T. J. Role of macrophage-derived lipoprotein lipase in lipoprotein metabolism and atherosclerosis. Arterioscler Thromb Vasc Biol 20, E53-62 (2000).

23 Lindqvist, P., Ostlund-Lindqvist, A. M., Witztum, J. L., Steinberg, D. \& Little, J. A. The role of lipoprotein lipase in the metabolism of triglyceride-rich lipoproteins by macrophages. J Biol Chem 258, 9086-9092 (1983).

24 O'Brien, K. D., Gordon, D., Deeb, S., Ferguson, M. \& Chait, A. Lipoprotein lipase is synthesized by macrophage-derived foam cells in human coronary atherosclerotic plaques. J Clin Invest 89, 1544-1550, doi:10.1172/JCI115747 (1992).

25 Mead, J. R., Irvine, S. A. \& Ramji, D. P. Lipoprotein lipase: structure, function, regulation, and role in disease. J Mol Med (Berl) 80, 753-769, doi:10.1007/s00109-002-0384-9 (2002).

26 Huang, S. C. et al. Cell-intrinsic lysosomal lipolysis is essential for alternative activation of macrophages. Nat Immunol 15, 846-855, doi:10.1038/ni.2956 (2014).

27 Vats, D. et al. Oxidative metabolism and PGC-1beta attenuate macrophagemediated inflammation. Cell Metab 4, 13-24, doi:10.1016/j.cmet.2006.05.011 (2006).

28 Suratt, B. T. et al. Neutrophil maturation and activation determine anatomic site of clearance from circulation. Am J Physiol Lung Cell Mol Physiol 281, L913-921, doi:10.1152/ajplung.2001.281.4.L913 (2001).

29 Athens, J. W. et al. Leukokinetic studies. IV. The total blood, circulating and marginal granulocyte pools and the granulocyte turnover rate in normal subjects. J Clin Invest 40, 989-995, doi:10.1172/JCI104338 (1961).

30 Basu, S., Hodgson, G., Katz, M. \& Dunn, A. R. Evaluation of role of G-CSF in the production, survival, and release of neutrophils from bone marrow into circulation. Blood 100, 854-861, doi:10.1182/blood.v100.3.854 (2002).

31 Dancey, J. T., Deubelbeiss, K. A., Harker, L. A. \& Finch, C. A. Neutrophil kinetics in man. J Clin Invest 58, 705-715, doi:10.1172/JCI108517 (1976).

32 Brinkmann, V. et al. Neutrophil extracellular traps kill bacteria. Science 303, 1532-1535, doi:10.1126/science.1092385 (2004).

33 Megens, R. T. A. et al. Presence of luminal neutrophil extracellular traps in atherosclerosis. Thrombosis and Haemostasis 107, 597-598, doi:10.1160/TH1109-0650 (2012).

34 Oklu, R., Stone, J. R., Albadawi, H. \& Watkins, M. T. Extracellular traps in lipid-rich lesions of carotid atherosclerotic plaques: implications for lipoprotein retention 
and lesion progression. J Vasc Interv Radiol 25, 631-634, doi:10.1016/j.jvir.2013.12.567 (2014).

35 Quillard, T. et al. TLR2 and neutrophils potentiate endothelial stress, apoptosis and detachment: implications for superficial erosion. Eur Heart J 36, 1394-1404, doi:10.1093/eurheartj/ehv044 (2015).

36 Naruko, T. et al. Neutrophil infiltration of culprit lesions in acute coronary syndromes. Circulation 106, 2894-2900, doi:10.1161/01.cir.0000042674.89762.20 (2002).

37 Pertiwi, K. R. et al. Neutrophil Extracellular Traps Participate in All Different Types of Thrombotic and Haemorrhagic Complications of Coronary Atherosclerosis. Thromb Haemost 118, 1078-1087, doi:10.1055/s-0038-1641749 (2018).

38 Warnatsch, A., Ioannou, M., Wang, Q. \& Papayannopoulos, V. Inflammation. Neutrophil extracellular traps license macrophages for cytokine production in atherosclerosis. Science 349, 316-320, doi:10.1126/science.aaa8064 (2015).

39 Westerterp, M. et al. Cholesterol Efflux Pathways Suppress Inflammasome Activation, NETosis, and Atherogenesis. Circulation 138, 898-912, doi:10.1161/CIRCULATIONAHA.117.032636 (2018).

40 Liu, Y. et al. Myeloid-Specific Deletion of Peptidylarginine Deiminase 4 Mitigates Atherosclerosis. Front Immunol 9, 1680, doi:10.3389/fimmu.2018.01680 (2018).

41 Drechsler, M., Megens, R. T., van Zandvoort, M., Weber, C. \& Soehnlein, 0. Hyperlipidemia-triggered neutrophilia promotes early atherosclerosis. Circulation 122, 1837-1845, doi:10.1161/CIRCULATIONAHA.110.961714 (2010).

42 van Leeuwen, M. et al. Accumulation of myeloperoxidase-positive neutrophils in atherosclerotic lesions in LDLR-/- mice. Arterioscler Thromb Vasc Biol 28, 84-89, doi:10.1161/ATVBAHA.107.154807 (2008).

43 Rotzius, P. et al. Distinct infiltration of neutrophils in lesion shoulders in ApoE-/mice. Am J Pathol 177, 493-500, doi:10.2353/ajpath.2010.090480 (2010).

44 Zernecke, A. et al. Protective role of CXC receptor 4/CXC ligand 12 unveils the importance of neutrophils in atherosclerosis. Circ Res 102, 209-217, doi:10.1161/CIRCRESAHA.107.160697 (2008).

45 Moulding, D. A., Quayle, J. A., Hart, C. A. \& Edwards, S. W. Mcl-1 expression in human neutrophils: regulation by cytokines and correlation with cell survival. Blood 92, 2495-2502 (1998).

46 Colotta, F., Re, F., Polentarutti, N., Sozzani, S. \& Mantovani, A. Modulation of granulocyte survival and programmed cell death by cytokines and bacterial products. Blood 80, 2012-2020 (1992).

47 Zhang, D. et al. Neutrophil ageing is regulated by the microbiome. Nature 525, 528-532, doi:10.1038/nature15367 (2015).

48 Jorch, S. K. \& Kubes, P. An emerging role for neutrophil extracellular traps in noninfectious disease. Nat Med 23, 279-287, doi:10.1038/nm.4294 (2017).

49 Soehnlein, O., Steffens, S., Hidalgo, A. \& Weber, C. Neutrophils as protagonists and targets in chronic inflammation. Nat Rev Immunol 17, 248-261, doi:10.1038/nri.2017.10 (2017).

50 Distel, E. et al. miR33 inhibition overcomes deleterious effects of diabetes mellitus on atherosclerosis plaque regression in mice. Circ Res 115, 759-769, doi:10.1161/CIRCRESAHA.115.304164 (2014). 
51 Barrett, T. J. et al. Apolipoprotein AI) Promotes Atherosclerosis Regression in Diabetic Mice by Suppressing Myelopoiesis and Plaque Inflammation. Circulation 140, 1170-1184, doi:10.1161/CIRCULATIONAHA.119.039476 (2019).

52 Hilgendorf, I. \& Swirski, F. K. Making a difference: monocyte heterogeneity in cardiovascular disease. Curr Atheroscler Rep 14, 450-459, doi:10.1007/s11883012-0274-8 (2012).

53 Wong, S. L. et al. Diabetes primes neutrophils to undergo NETosis, which impairs wound healing. Nat Med 21, 815-819, doi:10.1038/nm.3887 (2015).

54 Menegazzo, L. et al. NETosis is induced by high glucose and associated with type 2 diabetes. Acta Diabetol 52, 497-503, doi:10.1007/s00592-014-0676-x (2015).

55 Nagareddy, P. R. et al. Hyperglycemia promotes myelopoiesis and impairs the resolution of atherosclerosis. Cell Metab 17, 695-708, doi:10.1016/j.cmet.2013.04.001 (2013).

56 Yuan, C. et al. Human Aldose Reductase Expression Prevents Atherosclerosis Regression in Diabetic Mice. Diabetes 67, 1880-1891, doi:10.2337/db18-0156 (2018).

57 Fuchs, T. A. et al. Novel cell death program leads to neutrophil extracellular traps. J Cell Biol 176, 231-241, doi:10.1083/jcb.200606027 (2007).

58 Yamamoto, K. et al. Augmented neutrophil extracellular traps formation promotes atherosclerosis development in socially defeated apoE(-/-) mice. Biochem Biophys Res Commun 500, 490-496, doi:10.1016/j.bbrc.2018.04.115 (2018).

59 Mogayzel, P. J., Jr. et al. Cystic fibrosis pulmonary guidelines. Chronic medications for maintenance of lung health. Am J Respir Crit Care Med 187, 680-689, doi:10.1164/rccm.201207-1160oe (2013).

60 Awasthi, D. et al. Oxidized LDL induced extracellular trap formation in human neutrophils via TLR-PKC-IRAK-MAPK and NADPH-oxidase activation. Free Radic Biol Med 93, 190-203, doi:10.1016/j.freeradbiomed.2016.01.004 (2016).

61 Yipp, B. G. et al. Infection-induced NETosis is a dynamic process involving neutrophil multitasking in vivo. Nat Med 18, 1386-1393, doi:10.1038/nm.2847 (2012).

62 Napirei, M., Wulf, S., Eulitz, D., Mannherz, H. G. \& Kloeckl, T. Comparative characterization of rat deoxyribonuclease 1 (Dnase1) and murine deoxyribonuclease 1-like 3 (Dnase113). Biochem J 389, 355-364, doi:10.1042/BJ20042124 (2005).

63 Jimenez-Alcazar, M. et al. Host DNases prevent vascular occlusion by neutrophil extracellular traps. Science 358, 1202-1206, doi:10.1126/science.aam8897 (2017).

64 Peled, M. et al. A wild-type mouse-based model for the regression of inflammation in atherosclerosis. PLoS One 12, e0173975, doi:10.1371/journal.pone.0173975 (2017).

65 Gray, R. D., McCullagh, B. N. \& McCray, P. B. NETs and CF Lung Disease: Current Status and Future Prospects. Antibiotics (Basel) 4, 62-75, doi:10.3390/antibiotics4010062 (2015).

66 Soehnlein, O., Ortega-Gomez, A., Doring, Y. \& Weber, C. Neutrophil-macrophage interplay in atherosclerosis: protease-mediated cytokine processing versus NET release. Thromb Haemost 114, 866-867, doi:10.1160/TH15-08-0623 (2015). 
67 Kolaczkowska, E. et al. Molecular mechanisms of NET formation and degradation revealed by intravital imaging in the liver vasculature. Nat Commun 6, 6673, doi:10.1038/ncomms7673 (2015).

68 Hakkim, A. et al. Impairment of neutrophil extracellular trap degradation is associated with lupus nephritis. Proc Natl Acad Sci U S A 107, 9813-9818, doi:10.1073/pnas.0909927107 (2010).

69 Beiter, T. et al. Neutrophils release extracellular DNA traps in response to exercise. J Appl Physiol (1985) 117, 325-333, doi:10.1152/japplphysiol.00173.2014 (2014).

70 Kumamoto, T. et al. in European Heart Journal Vol. 27 2081-2087 (2006).

71 Yasutomo, K. et al. Mutation of DNASE1 in people with systemic lupus erythematosus. Nat Genet 28, 313-314, doi:10.1038/91070 (2001).

72 Bodano, A., Amarelo, J., Gonzalez, A., Gomez-Reino, J. J. \& Conde, C. Novel DNASE I mutations related to systemic lupus erythematosus. Arthritis Rheum 50, 40704071, doi:10.1002/art.20721 (2004).

73 Zhu, B. et al. Increased DNase I activity in diabetes might be associated with injury of pancreas. Mol Cell Biochem 393, 23-32, doi:10.1007/s11010-014-20431 (2014).

74 Park, J. et al. Cancer cells induce metastasis-supporting neutrophil extracellular DNA traps. Sci Transl Med 8, 361ra138, doi:10.1126/scitranslmed.aag1711 (2016).

75 Schonfelder, T., Jackel, S. \& Wenzel, P. Mouse models of deep vein thrombosis. Gefasschirurgie 22, 28-33, doi:10.1007/s00772-016-0227-6 (2017).

76 Chitrabamrung, S., Rubin, R. L. \& Tan, E. M. Serum deoxyribonuclease I and clinical activity in systemic lupus erythematosus. Rheumatol Int 1, 55-60, doi:10.1007/bf00541153 (1981). 
Valorisation 


\section{Valorisation}

Type1 \& Type2 Diabetes Mellitus (T1DM, T2DM) are common diseases with increasing prevalence worldwide. ${ }^{1}$ Globally, 425 million people are diagnosed with diabetes with an estimated cost of $\sim \$ 727$ billion. ${ }^{2}$ Global incidence for diabetes is projected to rise to $\$ 629$ million by 2045 . In the United States alone, with >30 million diagnosed patients, diabetes is the most expensive chronic disease, reaching an economic burden of $\$ 327$ billion. $^{3}$ In the Netherlands, about $6.5 \%$ of the Dutch population has been diagnosed with diabetes ${ }^{4}$ with projection to rise to $8 \%$ by $2025^{5}$ and yearly costs of $€ 6.8$ billion ${ }^{4}$. These costs not only include direct costs of treating the disease, e.g. medication, but also costs of treating its complications, such as cardiovascular diseases (CVDs). Subjects suffering from diabetes have a significantly increased risk of atherosclerosis and associated CV events. ${ }^{6}$ In the US, cost of treatment of $\mathrm{CV}$-related in diabetics is estimated at $\$ 37.3$ billion. $^{7}$ In the Netherlands, about $30 \%$ of the people with diabetes are suffering from CVD, and the treatment of CVD in those diabetes patients makes up $\sim € 1$ billion of the total economic burden of diabetes. CVD is, after end-stage renal disease, the most costly complications of diabetics in the Netherlands. ${ }^{4}$

$\underline{\text { CVD }}$ is the deadliest disease worldwide. Despite a steady decline in death from CVDs due to advances in scientific discoveries, clinical cardiology, as well as public health, it is still responsible for $30 \%$ of deaths. ${ }^{8}$ The global cost is estimated to be $\$ 863$ billion and is predicted to increase to $\$ 1044$ billion until $2030^{9}$ due to a rising and aging population and the high incidence of secondary complications after survival of a primary CVD incident. The increased risk of patients with diabetes to suffer from CVD is due to the fact that metabolic risk factors for atherosclerotic CVD are commonly found in subjects with diabetes. Hyperglycemia is an independent risk factor for CVD, next to genetic predisposition, hypertension and diabetic dyslipidemia. The adjusted relative risk for T2DM compared to non-T2DM patients is 1.7 for cardiovascular death, 1.8 for myocardial infarction, and 1.5 for stroke. ${ }^{10}$

Strikingly, individuals suffering from diabetes die mostly from CVD complications rather than diabetes itself. In the US, a total of 277,000 individuals with diabetes died in 2017 of which for 111,000 CVD was the primary cause of death. ${ }^{7}$ In the Netherlands, mortality rates of CVD have halved over the past two decades and with $26 \%$, it holds 
second place of the most non-communicable diseases, right after cancer $(32 \%) .{ }^{11,12}$ Interestingly, diabetes takes with $2 \%$ the $4^{\text {th }}$ place, and although CVD mortality has declined, the increase in diabetes has reversed positive trends and represents a major challenge to further prevent CVD.

The underlying mechanisms of increased CVD risk in individuals with diabetes is still unclear and current treatment focuses on treating hyperglycemia in diabetic patients, followed by statins in case of elevated LDL cholesterol (LDL-C) levels. Nonetheless, even if both are treated, diabetes patients still have a $\sim 2$ to 4-fold increased CVD risk compared to non-diabetics. Other components that potentially lead to the increased risk, such as elevated triglycerides (TG) levels and low HDL cholesterol (HDL-C) levels, are not being treated due to lack of evidence. While experimental studies of raising HDL documented benefits ${ }^{13,14}$, clinical trials raising HDL-C have mostly failed to show beneficial CVD outcome ${ }^{15,16}$ and other HDL functions are currently explored. Experimental studies focusing on hypertriglyceridemia (HyperTG) and atherosclerosis are limited, and clinical trials of TG-lowering drugs and CVD risk are currently ongoing.

Our research has focused on the two comorbidities of diabetes, i.e. elevated plasma TG levels accompanied by low HDL-C and hyperglycemia-related neutrophilia/Neutrophil extracellular traps (NET) formation. The aim was to elucidate the importance of both in atherosclerosis regression to shed light into possible new treatment options and thereby lower the residual CVD risk in diabetics.

\section{Valorisation of key findings of this thesis}

The results from our pre-clinical models showed that elevated TG levels do not lead to impaired atherosclerosis regression after LDL-C lowering, despite significantly reduced HDL-C and HDL particles (HDL-Ps). This might be due maintained cholesterol efflux capacity (CEC) - the considered key-antiatherogenic HDL function.

Further, we found that clearance of NETs using DNase1 can overcome diabetesimpaired regression despite ongoing hyperglycemia, due to reduction in inflammation. This suggested that NETs contribute to the increased CVD risk in this population. Further, given that DNase1 (Pulmozyme ${ }^{\circledR}$ ) is already clinically used for patients with cystic fibrosis ${ }^{17}$, these results also suggest a potential treatment for NET reduction. 


\section{Potential of TG lowering drugs to reduce residual CVD risk of diabetics}

Effective TG-lowering drugs are available and are used in patients with TG levels $>500 \mathrm{mg} / \mathrm{dl}$ to reduce the risk of pancreatitis. ${ }^{18-21}$ However, guidelines on using TG-lowering drugs to reduce CVD risk are not clearly defined due to lack of evidence. European Guidelines (ESC/EAS guidelines for the management of dyslipidemias ${ }^{22}$ and European guidelines on CVD prevention in clinical practice ${ }^{23}$ ) recommend that individuals with TG levels $>1.7 \mathrm{mmol} / \mathrm{l}(>150 \mathrm{mg} / \mathrm{dl}$ ) should be considered at increased risk of CVD, but did not include risk estimation of TG or recommended target values. Earlier guidelines from the adult treatment panel are in line with the European guidelines ${ }^{24}$, but more recent guidelines from the ACC/AHA on the assessment of CVD risk do not mention TGs at all. ${ }^{25}$ There is evidence suggesting that reducing TG could be beneficial for CVD, especially in individuals with preexisting coronary heart disease and diabetes. ${ }^{24,26-28}$ But one of the key problems is that clinical trials that appear to confirm CVD benefits of TG-lowering therapy rely on subgroup analyses, and trials designed to focus on subjects with hyperTG only were missing. Thus, these results are non-conclusive.

The REDUCE-IT trial was the first published intervention trial to determine the relationship between TG and atherosclerosis in a population with elevated plasma TG levels. Patients at high risk for CVD and TG levels $\geq 135 \mathrm{mg} / \mathrm{dL}$ and $<500 \mathrm{mg} / \mathrm{dL}$ were prescribed icosapent-ethyl (a highly purified eicosapentaenoic acid; EPA) over a median time period of 4.9 years and showed reduced CVD risk including first, subsequent, and total ischemic events. ${ }^{29,30}$ However, it is still unclear if the reduction in ischemic events was due to lowering TG or due to a combination of effects involving plaque instability reduction and inflammation. Notably, results were consistent among diabetic patients. Another trial looking at the effect of icosapent ethyl on progression of coronary atherosclerosis in patients with elevated TG levels on Statin therapy EVAPORATE - might help to explain the cardiovascular benefit noted in the REDUCEIT trial, and the 9 months interim results show reduction in total plaque volume measured as coronary CTA (computed tomography angiogram), but no reduction in low attenuation plaque volume. ${ }^{31,32}$ Further, intense research is being done on the mechanisms of action for omega-3 fatty acids (reviewed in ${ }^{33}$ ).

Less exciting was the STRENGTH Trial, which studied the effect of Epanova (omega-3 carboxylic acids) on CVD risk reduction in $\sim 13.000$ patients with mixed 
dyslipidemia (increased TG, low HDL-C) and increased risk of CVD. ${ }^{34}$ The Trial has been discontinued earlier this year (January 2020) due to the unlikelihood of demonstrating a benefit to patients. ${ }^{35}$

There are currently further clinical trials ongoing that are looking at the effect of TG-lowering therapy in high-risk CVD subjects, including the PROMINENT Trial (Pemafibrate to reduce CV outcomes by reducing TG in patients with diabetes), with results being expected over the next 1-2 years (reviewed $\mathrm{in}^{36}$ ).

\section{Potential of targeting NETs}

Two possible therapeutic targets to reduce NETs in atherosclerosis have been studied in mice - 1. PAD4 inhibition and 2. DNase1 treatment. ${ }^{37}$ In our studies, we focused on DNase1, because it is clinically already used to treat cystic fibrosis. DNase1 (Pulmozyme ${ }^{\circledR}$ ) has been approved by the FDA (Federal Drug Administration) >25 years ago, is on the market in $>65$ countries and did not lead to any signs of major toxicity or detrimental long-term effects. Excitingly, the positive effects of DNase1 in treating cystic fibrosis has been proposed to be partly due to NETs degradation. ${ }^{38}$ Our, as well as other studies ${ }^{39-41}$ showed that NETs degradation via additional intraperitoneal (i.p.) DNase1 administration is beneficial in the context of CVD in mice. In humans, however, i.p. administrations of DNase1 solely may compromise immunoprotective functions of NETs, e.g. antimicrobial activity. Cystic fibrosis patients take DNase1 via a nebulizer, but whether an effective dose of DNase1 would also reach atherosclerotic plaques, is questionable and rather unlikely. A better way to administrate DNase1 without compromising immunoprotective functions might be the use of DNase1-coated nanoparticles, which were shown to reduce size of lung metastases in mice via NETs degradation. ${ }^{42}$ Nano-based delivery systems have been proven to be safer and more effective than delivering a drug solely, with targeted delivery to site of disease, increased efficacy per drug dose and avoidance of off-target effects $^{43}$. Nanodrugs are clinically already used and are administered orally or intravenously ${ }^{44}$ to e.g. treat cancer, and have been shown to be beneficial for CVD in preclinical models (reviewed in ${ }^{45}$ ). Various types of nanoparticles are available (e.g. type IV collagen, cyclodextrin-based polysaccharides, lipid nanoparticles) and the correct therapeutic strategy with DNase1 as therapeutic agent needs to be tested in atherosclerotic mouse models. 
Despite the exciting potential of DNase1 treatment in (diabetic) atherosclerosis, NET research is still in its infancies and we are lacking enough evidence as well mechanistic details before therapeutically targeting NETs to lower CVD risk is clinically transferable.

\section{Translational capacity of the animal models}

To study the effect of elevated TG levels on atherosclerosis, we used an inducible lipoprotein lipase deficient mouse model ( $\left.i L L^{-1-}\right)$. Our mouse model had mild to moderate hyperTG with TG levels of $\sim 500 \mathrm{mg} / \mathrm{dL}$, which is significantly lower than that of $L \mathrm{pL}$ deficient patients whose TG levels reach $>1000 \mathrm{mg} / \mathrm{dL}$. While our mouse model also has TG levels $>1000 \mathrm{mg} / \mathrm{dL}$ when fed a high-fat diet, we chose to use a western diet resulting in mild-to-moderate hyperTG $(\sim 500 \mathrm{mg} / \mathrm{dL})$. These levels reflect $\mathrm{LpL}$ deficient patients on treatment and restricted diet as well as diabetics suffering from diabetic dyslipidemia. Further, subjects with genetic LpL deficiency are rare and not likely to impact genetic analysis of overall CVD risk associated with plasma TG levels. One limitation of our mouse model is the lack of lipolysis, which has been postulated together with hyperTG to be the cause for CVD risk by creating high concentrations of toxic lipids and remnant lipoproteins. To overcome this limitation, we have included mice with LpL expression in macrophages or endothelial cells. These studies led to the same results, showing that hyperTG does not impair atherosclerosis regression after LDL-C lowering.

Another limitation of our mouse model is the lack of cholesterol ester transfer protein (CETP) expression in mice. To humanize the lipoprotein metabolism in mice, we have introduced hCETP and again, confirmed that hyperTG + hCETP does not impair atherosclerosis regression after lipid lowering. Overall, we think our mouse model can be translated to the human condition, which we underline with the human data.

To study the effect of hyperglycemia-induced neutrophilia and NET formation, we used Ldlr/r mice injected with streptazotocin (STZ). STZ is a glucosamine-nitrosourea compound (glucose analogue) that is preferentially toxic towards insulin-producing beta cells. ${ }^{46}$ We used a low dose $(0.05 \mathrm{mg} / \mathrm{g} \mathrm{BW})$ over five consecutive days leading to cytotoxic effects in the pancreas resulting in pancreatic islet inflammation, insulin deficiency and hypergylcemia. ${ }^{46,47}$ Thereby, this method mimics human T1DM, while 
the majority of individuals with diabetes are diagnosed with $\mathrm{T}_{2} \mathrm{DM}^{48}$. Further, compared to severe systemic inflammation in mice, human leukocytosis is almost always mild. Therefore, translation from the animal model to human situation has to be done with caution.

To further study the role of endonucleases in atherosclerotic plaques, we used different DNase1 knock-out (KO) mouse models - DNase1 and DNase1L3 single KO, and DNase1/1L3 double KO mice. DNase1 and DNase1L3 single KO mouse models are commonly used to study systemic lupus erythematosus (SLE). For DNase ${ }^{\text {- }}$ mice, it takes 9 months to develop the SLE phenotype (increase in circulating Anti-Nuclear Antibodies (ANA), as well as antibodies against double-stranded DNA, histones, and Smith proteins). ${ }^{49}$ At 12 month of age, these mice develop severe nephritis and kidney damage. DNase $1 \mathrm{~L}^{\text {-/ }}$ can develop SLE as early as 5 weeks of age. ${ }^{50}$ The double KO mice were created by our collaborators and not much is published about their phenotype. Therefore, DNase 1\% mice are suitable for non-SLE experiments shorter than 9 months, while results from $D N a s e 1 L 3^{-/}$and the double $\mathrm{KO}$ mice might be confounded. In our performed 20 to 24 weeks long atherosclerotic study, we did not observe significant differences between our mouse models regarding NET formation, atherosclerosis progression and regression. Further, we did not detect any physical or behavioral abnormalities as well as no signs for major inflammation or tissue damage. Notably, we did not test for SLE markers.

Using administration of additional DNase1 results in beneficial effects on NET formation and atherosclerosis regression in especially diabetic mice and it is of interest to determine if the opposite effect can be observed using the various DNase KO mouse models. However, a study reported that double $\mathrm{KO}$ mice die within days after induction of severe neutrophilia. ${ }^{51}$ Thus, using the double $\mathrm{KO}$ mice in a diabetic atherosclerosis study (shown to induce neutrophilia) might not be feasible. In conclusion, the translation of results from the various atherosclerotic DNase $\mathrm{KO}$ mouse models to humans is challenging. 


\section{Conclusion}

Overall, our results show that treatment of hyperglycemia-associated inflammation seems to be superior over treating hyperTG, especially in individuals whose diabetes is not well controlled.

Although effective TG-reducing treatments are available, our results do not suggest benefits in CVD prevention. In agreement, the results of two recent interventional trials - REDUCE-IT \& STRENGTH, studying the potential benefits of TG-lowering therapy in high CVD-risk patients, were rather disappointing. With research on the mechanisms behind EPA treatment as well as results from ongoing TG-lowering trials being on their way, we will hopefully get an answer to decide if lowering elevated TGs should be included in the CVDs treatment guidelines.

While relative lack of evidence and knowledge does not allow clinical translation of NET targeting therapeutics in CVD as of yet, rapidly evolving research holds promise for their clinical translation in the near future. 


\section{References}

$1 \quad$ Vol. 97888 (World Health Organization, 2016).

2 Federation, I. D. Diabetes facts \& figures, $<$ https://www.idf.org/aboutdiabetes/what-is-diabetes/facts-figures.html> (2017).

3 Dall, T. M. et al. The Economic Burden of Elevated Blood Glucose Levels in 2017: Diagnosed and Undiagnosed Diabetes, Gestational Diabetes Mellitus, and Prediabetes. Diabetes Care 42, 1661-1668, doi:10.2337/dc18-1226 (2019). Peters, M. L., Huisman, E. L., Schoonen, M. \& Wolffenbuttel, B. H. R. The current total economic burden of diabetes mellitus in the Netherlands. Neth J Med 75, 281-297 (2017).

5 Baan, C. A. et al. [Diabetes mellitus in the Netherlands: estimate of the current disease burden and prognosis for 2025]. Ned Tijdschr Geneeskd 153, A580 (2009).

6 Low Wang, C. C., Hess, C. N., Hiatt, W. R. \& Goldfine, A. B. Clinical Update: Cardiovascular Disease in Diabetes Mellitus: Atherosclerotic Cardiovascular Disease and Heart Failure in Type 2 Diabetes Mellitus - Mechanisms, Management, and Clinical Considerations. Circulation 133, 2459-2502, doi:10.1161/CIRCULATIONAHA.116.022194 (2016).

7 American Diabetes, A. Economic Costs of Diabetes in the U.S. in 2017. Diabetes Care 41, 917-928, doi:10.2337/dci18-0007 (2018).

8 Nabel, E. G. \& Braunwald, E. A tale of coronary artery disease and myocardial infarction. $N$ Engl J Med 366, 54-63, doi:10.1056/NEJMra1112570 (2012).

9 Bloom, D. E., Cafiero, E.T., Jané-Llopis, E., Abrahams-Gessel, S., Bloom, L.R., Fathima, S., Feigl, A.B., Gaziano, T., Mowafi, M., Pandya, A., Prettner, K., Rosenberg, L., Seligman, B., Stein, A., \& Weinstein, C. The Global Economic Burden of Noncommunicable Diseases, <http://apps.who.int/medicinedocs/documents/s18806en/s18806en.pdf> (2011).

10 Dhindsa, D. S., Sandesara, P. B. \& Shapiro, M. D. The Intersection of Diabetes and Cardiovascular Disease-A Focus on New Therapies. Front Cardiovasc Med 5, 160, doi:10.3389/fcvm.2018.00160 (2018).

11 Koopman, C. et al. Explaining the Decline in Coronary Heart Disease Mortality in the Netherlands between 1997 and 2007. PLoS One 11, e0166139, doi:10.1371/journal.pone.0166139 (2016).

12 WHO. Non-communicable diseases (NCD) country profile The Netherlands, 2014. (2014).

13 Feig, J. E. et al. HDL promotes rapid atherosclerosis regression in mice and alters inflammatory properties of plaque monocyte-derived cells. Proc Natl Acad Sci U S A 108, 7166-7171, doi:10.1073/pnas.1016086108 (2011).

14 Hewing, B. et al. Effects of native and myeloperoxidase-modified apolipoprotein a-I on reverse cholesterol transport and atherosclerosis in mice. Arterioscler Thromb Vasc Biol 34, 779-789, doi:10.1161/ATVBAHA.113.303044 (2014).

15 Group, H. T. C. et al. Effects of extended-release niacin with laropiprant in highrisk patients. $N$ Engl J Med 371, 203-212, doi:10.1056/NEJMoa1300955 (2014).

16 Barter, P. J. et al. Cholesteryl ester transfer protein: a novel target for raising HDL and inhibiting atherosclerosis. Arterioscler Thromb Vasc Biol 23, 160-167, doi:10.1161/01.atv.0000054658.91146.64 (2003). 
17 Mogayzel, P. J., Jr. et al. Cystic fibrosis pulmonary guidelines. Chronic medications for maintenance of lung health. Am J Respir Crit Care Med 187, 680-689, doi:10.1164/rccm.201207-1160oe (2013).

18 Jacobson, T. A. et al. National Lipid Association recommendations for patientcentered management of dyslipidemia: part 1 - executive summary. J Clin Lipidol 8, 473-488, doi:10.1016/j.jacl.2014.07.007 (2014).

19 Preiss, D. et al. Lipid-modifying therapies and risk of pancreatitis: a metaanalysis. JAMA 308, 804-811, doi:10.1001/jama.2012.8439 (2012).

20 Murphy, M. J., Sheng, X., MacDonald, T. M. \& Wei, L. Hypertriglyceridemia and acute pancreatitis. JAMA Intern Med 173, 162-164, doi:10.1001/2013.jamainternmed.477 (2013).

21 Lindkvist, B., Appelros, S., Regner, S. \& Manjer, J. A prospective cohort study on risk of acute pancreatitis related to serum triglycerides, cholesterol and fasting glucose. Pancreatology 12, 317-324, doi:10.1016/j.pan.2012.05.002 (2012).

22 European Association for Cardiovascular, P. et al. ESC/EAS Guidelines for the management of dyslipidaemias: the Task Force for the management of dyslipidaemias of the European Society of Cardiology (ESC) and the European Atherosclerosis Society (EAS). Eur Heart J 32, 1769-1818, doi:10.1093/eurheartj/ehr158 (2011).

23 Perk, J. et al. European Guidelines on cardiovascular disease prevention in clinical practice (version 2012). The Fifth Joint Task Force of the European Society of Cardiology and Other Societies on Cardiovascular Disease Prevention in Clinical Practice (constituted by representatives of nine societies and by invited experts). Eur Heart J 33, 1635-1701, doi:10.1093/eurheartj/ehs092 (2012).

24 National Cholesterol Education Program Expert Panel on Detection, E. \& Treatment of High Blood Cholesterol in, A. Third Report of the National Cholesterol Education Program (NCEP) Expert Panel on Detection, Evaluation, and Treatment of High Blood Cholesterol in Adults (Adult Treatment Panel III) final report. Circulation 106, 3143-3421 (2002).

25 Goff, D. C., Jr. et al. 2013 ACC/AHA guideline on the assessment of cardiovascular risk: a report of the American College of Cardiology/American Heart Association Task Force on Practice Guidelines. Circulation 129, S49-73, doi:10.1161/01.cir.0000437741.48606.98 (2014).

26 Miller, M. et al. Triglycerides and cardiovascular disease: a scientific statement from the American Heart Association. Circulation 123, 2292-2333, doi:10.1161/CIR.0b013e3182160726 (2011).

27 Berglund, L. et al. Evaluation and treatment of hypertriglyceridemia: an Endocrine Society clinical practice guideline. J Clin Endocrinol Metab 97, 29692989, doi:10.1210/jc.2011-3213 (2012).

28 Brunzell, J. D. et al. Lipoprotein management in patients with cardiometabolic risk: consensus conference report from the American Diabetes Association and the American College of Cardiology Foundation. J Am Coll Cardiol 51, 1512-1524, doi:10.1016/j.jacc.2008.02.034 (2008).

29 Bhatt, D. L. et al. Effects of Icosapent Ethyl on Total Ischemic Events: From REDUCE-IT. J Am Coll Cardiol 73, 2791-2802, doi:10.1016/j.jacc.2019.02.032 (2019). 
30 Bhatt, D. L. et al. Cardiovascular Risk Reduction with Icosapent Ethyl for Hypertriglyceridemia. N Engl J Med 380, 11-22, doi:10.1056/NEJMoa1812792 (2019).

31 Budoff, M. J. Effect of Icosapent Ethyl on Progression of Coronary Atherosclerosis in Patients With Elevated Triglycerides on Statin Therapy - EVAPORATE, $<$ https://www.acc.org/latest-in-cardiology/clinicaltrials/2019/11/15/17/46/evaporate> (2019).

32 Neale, T. EVAPORATE Hints at Slowed Plaque Progression With Icosapent Ethyl, $<$ https://www.tctmd.com/news/evaporate-hints-slowed-plaque-progressionicosapent-ethyl> (2019).

33 Preston Mason, R. New Insights into Mechanisms of Action for Omega-3 Fatty Acids in Atherothrombotic Cardiovascular Disease. Curr Atheroscler Rep 21, 2, doi:10.1007/s11883-019-0762-1 (2019).

34 Outcomes Study to Assess STatin Residual Risk Reduction With EpaNova in HiGh CV Risk PatienTs With Hypertriglyceridemia (STRENGTH), $<$ https://clinicaltrials.gov/ct2/show/NCT02104817>(

35 Update on Phase III STRENGTH trial for Epanova in mixed dyslipidaemia, $<$ https://www.astrazeneca.com/media-centre/press-releases/2020/update-onphase-iii-strength-trial-for-epanova-in-mixed-dyslipidaemia-13012020.html> (2020).

36 Huet, F., Roubille, C. \& Roubille, F. Is hypertriglyceridemia atherogenic? Curr Opin Lipidol 30, 291-299, doi:10.1097/MOL.0000000000000622 (2019).

37 Jorch, S. K. \& Kubes, P. An emerging role for neutrophil extracellular traps in noninfectious disease. Nat Med 23, 279-287, doi:10.1038/nm.4294 (2017).

38 Gray, R. D., McCullagh, B. N. \& McCray, P. B. NETs and CF Lung Disease: Current Status and Future Prospects. Antibiotics (Basel) 4, 62-75, doi:10.3390/antibiotics4010062 (2015).

39 Warnatsch, A., Ioannou, M., Wang, Q. \& Papayannopoulos, V. Inflammation. Neutrophil extracellular traps license macrophages for cytokine production in atherosclerosis. Science 349, 316-320, doi:10.1126/science.aaa8064 (2015).

40 Yamamoto, K. et al. Augmented neutrophil extracellular traps formation promotes atherosclerosis development in socially defeated apoE(-/-) mice. Biochem Biophys Res Commun 500, 490-496, doi:10.1016/j.bbrc.2018.04.115 (2018).

41 Wong, S. L. et al. Diabetes primes neutrophils to undergo NETosis, which impairs wound healing. Nat Med 21, 815-819, doi:10.1038/nm.3887 (2015).

42 Park, J. et al. Cancer cells induce metastasis-supporting neutrophil extracellular DNA traps. Sci Transl Med 8, 361ra138, doi:10.1126/scitranslmed.aag1711 (2016).

43 Ventola, C. L. Progress in Nanomedicine: Approved and Investigational Nanodrugs. P T 42, 742-755 (2017).

44 Bobo, D., Robinson, K. J., Islam, J., Thurecht, K. J. \& Corrie, S. R. Nanoparticle-Based Medicines: A Review of FDA-Approved Materials and Clinical Trials to Date. Pharm Res 33, 2373-2387, doi:10.1007/s11095-016-1958-5 (2016). Flores, A. M. et al. Nanoparticle Therapy for Vascular Diseases. Arterioscler Thromb Vasc Biol 39, 635-646, doi:10.1161/ATVBAHA.118.311569 (2019).

46 Lenzen, S. The mechanisms of alloxan- and streptozotocin-induced diabetes. Diabetologia 51, 216-226, doi:10.1007/s00125-007-0886-7 (2008). 
47 Furman, B. L. Streptozotocin-Induced Diabetic Models in Mice and Rats. Curr Protoc Pharmacol 70, 547 41-45 47 20, doi:10.1002/0471141755.ph0547s70 (2015).

48 National Diabetes Statistics report. (Center for Disease Control and Prevention, 2017).

49 Kenny, E. F., Raupach, B., Abu Abed, U., Brinkmann, V. \& Zychlinsky, A. Dnase1deficient mice spontaneously develop a systemic lupus erythematosus-like disease. Eur J Immunol 49, 590-599, doi:10.1002/eji.201847875 (2019).

50 Sisirak, V. et al. Digestion of Chromatin in Apoptotic Cell Microparticles Prevents Autoimmunity. Cell 166, 88-101, doi:10.1016/j.cell.2016.05.034 (2016).

51 Jimenez-Alcazar, M. et al. Host DNases prevent vascular occlusion by neutrophil extracellular traps. Science 358, 1202-1206, doi:10.1126/science.aam8897 (2017). 
Acknowledgments 


\section{Acknowledgements}

It is done! Starting with my Bachelor in 2010, I never thought I would ever finish my studies with a $\mathrm{PhD}$, but 10 years later, here I am. Spending the past $4 \frac{1}{2}$ years on this thesis has been exciting as well as challenging and time was just flying by. I could have never done this on my own and would like to use the next few pages to acknowledge and thank people whose contribution was crucial for the completion of this thesis.

First, I would like to thank my Promoters Prof. Dr. Casper Schalkwijk and Prof. Dr. Edward A. Fisher, and co-promoter Dr. Kristiaan Wouters: Dear Casper, Dr. Fisher and Kristiaan, thank you for giving me the opportunity to be part of this joint, overseas $\mathrm{PhD}$ project and accompanying me through this whole adventure. The big advantage of a joint PhD project is to be able to spend time in two different labs, learn a variety of techniques and get double the input. This added a lot of value to not only my thesis work, but also to my development as a researcher.

Dear Casper, although I only spent a few months in your department, I fully enjoyed my time there. Your way of leading the department makes everyone feel welcome, and the relaxed and friendly atmosphere made it a pleasure to come to work every day. Thank you for the scientific input, the constructive feedback and the encouragements! Dear Dr. Fisher, I feel overly grateful to have spent 5 years under your supervision! Thank you, for taking a chance on me when I joined your lab as a masters student back in 2014 and for committing to me, when you offered me a spot in your lab as a PhD student. Thank you for providing me with a visa as well as a scholarship for the full 5 years and thereby giving me the chance to live in NYC and be part of a great scientific community. Thank you for the encouragements, your guidance, trust, time investment, overall support including your help in getting my own personal grant, and foremost, for sharing your scientific expertise. Although times were not always easy, I want to thank you for always pushing me beyond my limits and for your honest criticisms. You have taught me how to perform research rigorously and independently, and have shaped me into the researcher I am today. Furthermore, thank you for the stories about NYC and the states in general, as well as helping me to feel settled far away from home by taking me out for German food and beers. 
I would also like to thank Dr. Ira J. Goldberg, who was never officially one of my promoters, but truly felt like one. Thank you, Ira, for welcoming me in your research group, for believing in me and supporting my projects, for your positive energy, your enthusiasm, and for standing up for me and always being there when I had questions. Thank you also for your motivational encouragements and the tremendous help and fast feedback on written manuscripts.

Thank you both, Dr. Fisher and Ira, for your faith in me regarding these projects - I enjoyed working on it a lot and it is hard to put in words of how much I have learned and what your support meant to me. Both of you were constantly impressing me with your breadth of scientific knowledge, hard work, resilience and passion for science. You have been a great inspiration to me, and I feel very honored and proud to be one of your trainees!

Dear Kristiaan, from internship supervisor to co-promoter - I feel very lucky to have had your support for such a long time. Thank you for your commitment, believing in me, and always creating opportunities. Your energy and enthusiasm are truly infectious, and I loved working in your lab and under your supervision in general. Thank you for always seeing and showing me the positive view on results, even if they seemed negative first. Thank you for constructive feedback, ideas and suggestions; your creativity seems endless. Thank you also for your time investment, especially towards the corrections of my raw manuscript and thesis drafts. You have been an outstanding mentor - Thank You!

I would like to show appreciation towards my reading committee, Prof. Dr. C. Reutelingsperger, Prof. Dr. E.A.L. Biessen, Prof. Dr. M.P.J de Winther, Dr. M. Westerterp, and Dr. R. Koenen. Thank you for critically reading my thesis and the positive feedback!

A tremendous part of my PhD work would have not been possible without two former members of the Fisher lab - Drs. Jaume Amengual \& Tessa J Barrett. Dear Jaume and Tessa, you two were my go-to scientists for everything lab-related, and I would like to thank you both so much for supporting me and my research. Thank you for sharing your scientific knowledge and lab skills, for your constant advise, your patience 
and feedback! Jaume, thank you for not only supervising me during my master's thesis, but also the continuous help with my PhD project. Being in a lab with you was truly an adventure and next to the huge learning experience, also a lot of fun. Tessa, thank you for teaching me everything animal-related, for your guidance and answering the tons of questions I had, even after you moved on to the next lab.

I wish to express my gratitude towards Dr. Marleen van Greevenbroek. Dear Marleen, thank you for giving me the opportunity to include the CODAM study into my PhD Project, for teaching me SPSS, the constructive feedback and for helping me through my first first-author publication!

I would like to acknowledge our collaborators from the University of Washington, Prof. Dr. Karin E. Bornfeldt, Dr. Jay Heinecke, Dr. Jenny E. Kanter and especially Dr. Tomas Vaisar. Dear Tomas, thank you for conducting the HDL measurements and for your tremendous and quick help in understanding and analyzing the data. Specifically, I would also like to thank you for supporting my AHA predoctoral fellowship application.

I spent one month at Vanderbilt University, Nashville, TN and wish to show my appreciation towards Prof. dr. Kasey Vickers and the whole Vickers lab (especially Ryan, Cindy, Danielle, Carrie and Marisol). Thank you for the warm welcome, the hospitality, showing me around Nashville and its specialties (hot chicken!), and of course, for taking your time to teach me some of your methods. Although, the results unfortunately did not work out as planned, I did learn a lot and enjoyed my stay very much. Thank you, Kasey, for the continuous support after leaving your lab!

Further, I would like to thank my lab buddies Shruti, Prashanth, Tarik, Martin, and Monika -you made the lab a better place! Thank you, Shruti \& Prashanth, for your scientific advice and help, the fun lunch breaks, the in between coffee chats, the after lab drinks and Indian food. Thank you, Tarik, for the huge help with my animal colonies and for always being there to assist, if needed. I feel lucky to have shared an aisle with you, Martin \& Monika. I think we made a great connection between the Fisher and 
Moore lab. Thank you for your help and advice, especially regarding flow cytometry, FPLC and RNA Seq.

I would also like to acknowledge the previous and current Fisher lab \& Goldberg lab members including the staff members, especially Dr. Debapriya Basu, Jennifer DiMascio-Donohue, Julia Levine, Lesley-Ann Huggins, and Cecilia Ono. Many thanks also to the Reizis lab, specifically to Lee Serpas, for providing the DNase KO mice.

From colleagues to very close friends: Thank you, Maud and Alex!

Maud, I'm so happy we became such great friends and I enjoyed working, volunteering for INetNYC and spending free time together. I loved when we were living close to each other (even close for NYC terms) and I miss our Sunday brunches at your place, followed by yoga, the $\$ 1$ pizza slices after work plus all of our other fun activities (incl. seeing you fall asleep at the most random places ;)). Thank you also for being one of my paranymph! Although, it unfortunately won't be possible to have you by my side during the defense, you were by my side during the PhD and your support meant a lot! Alex, thank you for your help when you were still a member of the Fisher lab and for understanding and supporting me throughout the $\mathrm{PhD}$. I enjoyed our lunch breaks on the ferry, sharing bagels and looking at the skyline, as well as everything outside the lab. Thank you for bringing me closer to American "Culture" (Dunkin Donuts, Taco Bell, Chipotle, Chick-fil-A, etc. ;)), for not getting tired of teaching me new words or reexplaining them, always being open to try spots that I was reading about, even if it meant long a long commute for you, for dancing through the nights and so much more. I'm very happy we stayed friends even with the long NYC distance and am grateful for your visit in Maastricht and travelling to Spain together. I can't wait to welcome you in Europe soon!

This PhD work would have not been possible without support outside the lab.

I would like to thank two of my former roommates Janina and Line for making New York City feel like home. Housing in NYC is a real struggle and it was a relief to know that we had each other! Further, thank you both, for the emotional support, keeping up with me as well as supporting and distracting me in stressful times, and always being 
there for me. Janina, thank you for the amazing vacations, exploring not only NYC together, but also other parts of America during which we caught ourselves by being overly German more than once :D. Line, thank you for creating the perfect balance to work, starting from spontaneously going out for food and drinks at any time of the day or night, to going for a run in Brooklyn with an ice-cream stop in between. Thank you for our TT nights with cocktails and tea, strolls through Brooklyn, shopping sprees in SoHo, beach days, brunches and so on. It was a pleasure to have spent my last $1 \frac{1}{2}$ years in NYC living together with you!

Dear Mike, we have been friends since my $1^{\text {st }}$ year in NYC - something special in such a transient city. Thank you for all the good times we had (excluding Yoga ;)), and for being my weekend work buddy. I benefited from joining you at various coffee places for studying and always enjoyed all of our discussions. Thank you also for the language advise, the general support and encouraging words.

Dear Jenny, without you, my time in Maastricht wouldn't have been the same. Thank you for making me part of your life, including me in all the activities, for the hours of laughter, and for being my study buddy. Thank you also for the multiple visits in NYC and the great times in Canada.

Special thanks are going to my family, especially my parents and my grandparents. Meine liebe Familie, ich möchte euch von ganzen Herzen für die zahlreichen Besuche und die Post bedanken. Ihr habt mir immer das Gefühl gegeben, dass ich trotz der Entfernung dazu gehöre und, dass ich immer auf euch zählen kann. Liebe Mama, lieber Papa, ich möchte euch für die finanzielle und emotionale Unterstützung über die ganzen letzten 10 Jahre danken. Jetzt ist es offiziell - ich bin fertig mit studieren! Danke Papa, für die wöchentliche Post \& Telefongespräche und dein Vertrauen in mich. Danke, Mama und Uwe, dass ihr mich mindestens einmal im Jahr besucht und dadurch die Zeit zwischen meinen weihnachtlichen Besuchen verkürzt habt und an dich Mama, für die Gespräche und unterstützenden Worte. Vielen Dank auch euch, Oma \& Opa, dass ihr euch nochmal auf die große Reise nach New York begeben habt, um mich zu besuchen, sowie auch für die Post! 
Dear Isabell, thank you so much for the support over the past years! Talking to a close friend who is also a fellow PhD student has helped me so much and I couldn't have done it without you! Thank you for always being there for me, for listening, understanding and finding the right words to say. Although, we were in different countries and time zones, it felt like you were always there - we have spent many of my lunch breaks together on the phone, and even most of my time sectioning. I'm happy we managed to still visit and see each other on a yearly basis. I'm looking forward to being on the same continent again, including the many more (camping) trips ahead of us. It is an honor to have you by my side as a paranymph during the defense and I'm very grateful to have you as such a good friend! 
Curriculum Vitae 
Tatjana Josefs was born on the $6^{\text {th }}$ of June, 1990 in Bad Friedrichshall, Germany. She graduated from secondary school (Eckenberg-Gymnasium, Adelsheim, Germany) in 2009 followed by 9 months in New Zealand, where she worked as an au-pair and spent time travelling. In 2013, she received her bachelor's degree in nutritional sciences with focus on research from the Friedrich-Schiller University Jena, Germany. This was followed by a master's degree in biomedical sciences at the Maastricht University, the Netherlands. She performed internships in the department of Internal Medicine at Maastricht University, Maastricht, the Netherlands supervised by Dr. Kristiaan Wouters, and in the department of Cardiology at NYU School of Medicine, New York, USA, supervised by Prof. Dr. Edward A. Fisher. The master thesis was conducted in the Fisher lab entitled: ApoB100 on hepatic lipid droplets is associated with the autophagy factor p62, and was financially supported by a scholarship from the Dutch Heart Foundation (Hartstichting). In 2015, she received her master's degree with distinction and continued her work in the Fisher lab as a PhD student. The PhD project was conducted under the supervision of Prof Dr. Casper Schalkwijk (Maastricht University), Prof Dr. Edward A. Fisher (NYU School of Medicine), and Dr. Kristiaan Wouters (Maastricht University). During this time, she studied the role of diabetes-related factors, such as elevated circulating triglyceride levels, in atherosclerosis regression. This work was funded by a predoctoral fellowship from the American Heart Association and the results of her research are presented in this thesis. 
Scientific Output 


\section{List of Publications}

Josefs T*, Barrett TJ*, Brown EJ, Quezada A, Wu X, Voisin M, Amengual J, Fisher EA. Neutrophil Extracellular Traps (NETs) promote macrophage inflammation and impair atherosclerosis resolution in diabetic mice. JCI Insight. 2020;5(7):e134796. (IF:6.014)

Josefs T, Wouters K, Tietge UJF, Annema W, Dullaart RPF, Vaisar T, Arts ICW, van der Kallen CJH, Stehouwer CDA, Schalkwijk CG, Goldberg IJ, Fisher EA, van Greevenbroek MJ. High-density lipoprotein cholesterol efflux capacity is not associated with atherosclerosis and prevalence of cardiovascular outcome: The CODAM Study. J Clin Lipidol. 2020 Jan-Feb;14(1):122-132.e4. (IF: 3.860)

Chang HR*, Josefs T*, Scerbo D, Gumaste N, Hu Y, Huggins LA, Barrett TJ, Chiang SS, Grossman J, Bagdasarov S, Fisher EA, Goldberg IJ. Role of LpL (Lipoprotein lipase) in Macrophage Polarization In Vitro and In Vivo. Arterioscler Thromb Vasc Biol. 2019 Oct;39(10):1967-1985. (IF: 6.607)

Bijnen M, Josefs T, Cuijpers I, Maalsen CJ, van de Gaar J, Vroomen M, Wijnands E, Rensen SS, Greve JWM, Hofker MH, Biessen EAL, Stehouwer CDA, Schalkwijk CG, Wouters K. (2017). Adipose tissue macrophages induce hepatic neutrophil recruitment and macrophage accumulation in mice. Gut. $2018 \mathrm{Jul} ; 67(7): 1317-1327$. (IF: 16.658)

\section{Additional Manuscripts}

Josefs T, Basu D, Vaisar T, Arets B, Kanter JE, Huggins LA, Hu Y, Liu J, Heinecke $\mathrm{JW}$, Bornfeldt KE, Goldberg $\mathrm{IJ}^{*}$, Fisher $\mathrm{EA}^{*}$. Atherosclerosis regression and its relationship to cholesterol efflux capacity in hypertriglyceridemic mice.

In Revision

Voisin M, Shrestha E, Rollet C, Josefs T, Barrett TJ, Chang HR, Ruoff R, Nikain CA, Garabedian ML, Brown EJ, Goldberg IJ, Pineda-Torra I, Fisher EA, Garabedian MJ. Inhibiting bone marrow LXRa phosphorylation controls inflammation, atherosclerosis \& obesity. In Revision 


\section{Presentations}

2019 American Heart Association's Scientific Sessions; Philadelphia, PA, United States

- Oral presentation "Neutrophil Extracellular Traps (NETs) promote macrophage inflammation and contribute to diabetes-impaired atherosclerosis regression"

- Mediated Poster Presentation "Reduced HDL due to hypertriglyceridemia does not affect atherosclerosis regression"

- Invited Poster Presentation ATVB Early Career Networking Reception "Reduced HDL due to hypertriglyceridemia does not affect atherosclerosis regression"

2019 Gordon Research Conference Atherosclerosis; Newry, ME, United States

- Poster Presentation "Neutrophil Extracellular Traps (NETs) promote macrophage inflammation and contribute to diabetes-impaired atherosclerosis regression"

2018 Department of Medicine Research Day; New York, NY, United States

- Poster Presentation "High-density Lipoprotein Cholesterol Efflux Capacity is not associated with Atherosclerosis and Cardiovascular Events: The CODAM Study"

2018 Vascular Discovery: From Genes to Medicine 2018 Scientific Sessions (ATVB/PVD); San Francisco, CA, United States

- Poster Presentation "High-density Lipoprotein Cholesterol Efflux Capacity is not associated with Atherosclerosis and Cardiovascular Events: The CODAM Study" 
Scientific Output

\section{Awards and Grants}

- American Heart Association Predoctoral Fellowship; 18PRE33990436 (\$53.688)

- Poster Prize, Gordon Research Conference Atherosclerosis (\$250)

- Student Scholarship Hartstichting (Dutch Heart Foundation) (3.000€)

- Free Mover Grant, Maastricht University (2.000€) 
Abbreviations 


\section{Abbreviation}

AAM $\phi$

AAV

ABCA1/ABCG1

$\mathrm{AHA}$

apoA-I

apoB

ApoE-

ASO

AT

ATM

BMDM

BMI

$\mathrm{BP}$

CAD

CAM $\phi$

CD36

CE

CEC

CETP

$\mathrm{Cl}$

CIMT

CODAM

CRAMP

CVD

CVE

DNase1

DNase1L3

ECAR

EnD

FA

FAO

FFA

\section{Meaning}

alternatively activated macrophage

adeno-associated vector

ATP-binding cassette transporter A1/G1

American Heart Association

apolipoprotein A-I

apolipoprotein B

apolipoprotein E deficient

antisense oligonucleotides

adipose tissue

adipose tissue macrophage

bone marrow-derived macrophage

body mass index

blood pressure

Coronary Artery Disease

classically activated macrophages

cluster of differentiation 36

cholesteryl ester

cholesterol efflux capacity

cholesteryl ester transfer protein

confidence interval

carotid intima-media thickness

Cohort on Diabetes and Atherosclerosis Maastricht

cathelicidin-related antimicrobial peptide

Cardiovascular Disease

Cardiovascular Event

deoxyribonuclease 1

DNase1 like 3

extracellular acidification rate

endothelial dysfunction

fatty acid

fatty acid oxidation

free fatty acids 
FPG

H3Cit

$\mathrm{Hb} 1 \mathrm{Ac}$

hCETP

HDL

HDL-C

HDL-P

$\mathrm{HL}$

HMG-CoA

HyperTG

IDLs

IGM

IL

iNOS

IQR

LDL

LDL-C

LDLR

LdIIr-

LpL

LPM

Ly6G

MET

MI

MPO

Myh11

M $\phi$

$\mathrm{NADPH}$

NE

NETs

NGM

NLRP3

OA fasting plasma glucose

citrullinated $\mathrm{H} 3$

Hemoglobin A1c

human CETP

high-density lipoprotein

HDL cholesterol

HDL particle number

hepatic lipase

3-hydroxy-3-methylglutaryl coenzyme A

hypertriglyceridemia

intermediate-density lipoproteins

impaired glucose metabolism, prediabetes

interleukin

inducible nitric oxide synthase

Interquartile Range

low-density lipoprotein

LDL-Cholesterol

low-density lipoprotein receptor

low-density lipoprotein receptor deficient

lipoprotein lipase

large peritoneal macrophage

lymphocyte antigen 6 complex locus G6D

Metabolic Equivalent of Task

myocardial infarction

myeloperoxidase

myosin heavy chain-11

macrophage

nicotinamide adenine dinucleotide phosphatase

neutrophil elastase

neutrophil extracellular traps

normal glucose metabolism

NLR family pyrin domain-containing protein 3

oleic acid 
OCR

OR

oxLDL

PAD4

PCSK9

pDCs

PMA

PPAR

PR3

RCT

ROS

sdLDL,

SLE

SO

SPM

STZ

T1DM, T2DM

TG

TRL

VLDL

VSMC

WD

WT oxygen consumption rate

odds ratio

oxidized LDL

peptidylarginine deiminase 4

proprotein convertase subtilisin/kexin type 9

plasmacytoid dendritic cells

phorbol myristate acetate

peroxisome proliferator-activated receptor

proteinase 3

reverse cholesterol transport

reactive oxygen species

small dense LDL

lupus erythematous

sense oligonucleotide

small peritoneal macrophages

Streptozotocin

Type1 \& Type2 Diabetes Mellitus

triglyceride

TG-rich lipoproteins

very low-density lipoprotein

vascular smooth muscle cells

western diet

wild-type; C57BL/6 